\title{
Novel insights towards memory restoration
}

\author{
Citation for published version (APA):
}

Hescham, S-A. (2015). Novel insights towards memory restoration. [Doctoral Thesis, Maastricht University]. Maastricht University. https://doi.org/10.26481/dis.20151215sh

Document status and date:

Published: 01/01/2015

DOI:

10.26481/dis.20151215sh

Document Version:

Publisher's PDF, also known as Version of record

\section{Please check the document version of this publication:}

- A submitted manuscript is the version of the article upon submission and before peer-review. There can be important differences between the submitted version and the official published version of record.

People interested in the research are advised to contact the author for the final version of the publication, or visit the DOI to the publisher's website.

- The final author version and the galley proof are versions of the publication after peer review.

- The final published version features the final layout of the paper including the volume, issue and page numbers.

Link to publication

\footnotetext{
General rights rights.

- You may freely distribute the URL identifying the publication in the public portal. please follow below link for the End User Agreement:

www.umlib.nl/taverne-license

Take down policy

If you believe that this document breaches copyright please contact us at:

repository@maastrichtuniversity.nl

providing details and we will investigate your claim.
}

Copyright and moral rights for the publications made accessible in the public portal are retained by the authors and/or other copyright owners and it is a condition of accessing publications that users recognise and abide by the legal requirements associated with these

- Users may download and print one copy of any publication from the public portal for the purpose of private study or research.

- You may not further distribute the material or use it for any profit-making activity or commercial gain

If the publication is distributed under the terms of Article $25 \mathrm{fa}$ of the Dutch Copyright Act, indicated by the "Taverne" license above, 
NOVEL INSIGHTS TOWARDS MEMORY RESTORATION

Sarah-Anna Hescham 
(c) Sarah-Anna Hescham, Maastricht 2015

Novel insights towards memory restoration.

All rights are reserved. No part of this book may be reproduced or transmitted in any form or by any means, without permission in writing from the copyright holder. ISBN: 978-94-6169-789-9

Cover design: Paul Hanraets and Pim van Eijk

Typesetting and Layout: Optima Grafische Communicatie, Rotterdam

Production: Optima Grafische Communicatie, Rotterdam 


\title{
NOVEL INSIGHTS TOWARDS MEMORY RESTORATION
}

\author{
DISSERTATION
}

To obtain the degree of Doctor at Maastricht University, on the authority of the Rector Magnificus, Prof. dr. L.L.G. Soete, in accordance with the decision of the Board of Deans, to be defended in public on Tuesday 15th December 2015, at 16:00 hours by

Sarah-Anna Hescham 


\section{Supervisor:}

Prof. Dr. Y. Temel

\section{Co-supervisors:}

Dr. A. Blokland

Dr. A. Jahanshahi

\section{Assessment Committee:}

Prof. Dr. J.J. van Overbeeke (Chairman)

Dr. C. Hamani (Unversity of Toronto, Canada)

Prof. Dr. J. Kuhn (Johanniter Krankenhaus Oberhausen, Germany)

Dr. D.L.A. van den Hove

Prof. Dr. F. Verhey

Financial support for the publication of this dissertation was kindly provided by "Alzheimer Nederland", "Internationale Stichting Alzheimer Onderzoek (ISAO)" and the "School for Mental Health and Neuroscience, Maastricht University".
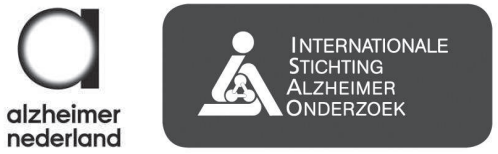
To my beloved grandmothers 



\section{LIST OF CONTENTS}

Chapter 1 General Introduction

STATE-OF-THE-ART

$\begin{array}{lll}\text { Chapter } 2 \text { Neuromodulation in psychiatric disorders } & 19\end{array}$

Chapter 3 Deep brain stimulation in dementia-related disorders 39

\section{MODELLING}

Chapter 4 A neuroanatomical analysis of the effects of a memory impairing dose of scopolamine in the rat brain using cytochrome c oxidase as principle marker

\section{BEHAVIORAL EFFECTS}

Chapter 5 Deep brain stimulation of the forniceal area enhances memory functions in experimental dementia: The role of stimulation parameters

Chapter 6 Deep brain stimulation in an aged animal model to restore memory functions

Chapter 7 Behavioral effects of deep brain stimulation of different structures within the Papez circuit on memory- and anxietyrelated functions

\section{MECHANISMS OF ACTION}

Chapter 8 Increased number of $\mathrm{TH}$-immunoreactive cells in the Ventral Tegmental Area after Deep Brain Stimulation of the Anterior Nucleus of the Thalamus

Chapter 9 Fornix deep brain stimulation enhances acetylcholine levels in the hippocampus

Chapter 10 Fornix deep brain stimulation induces long-term spatial memory independent of hippocampal neurogenesis

Chapter 11 General Discussion and Conclusion 



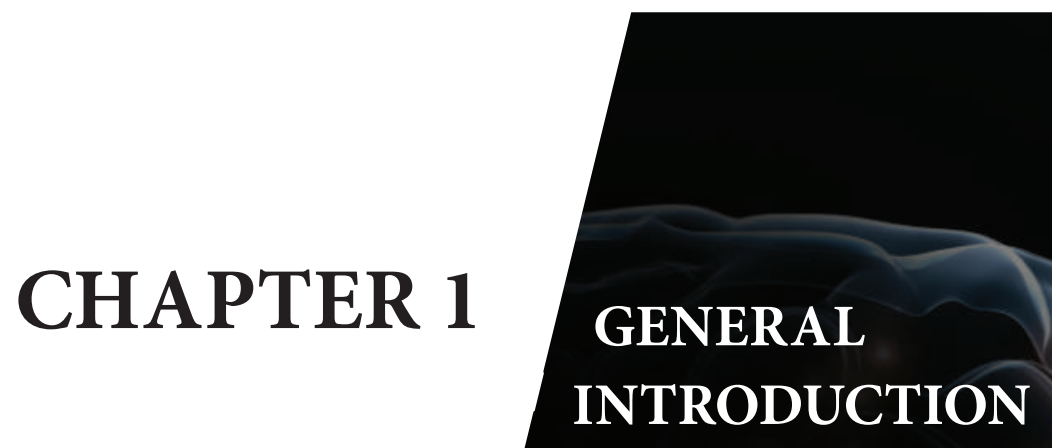





\section{MEMORY}

Memory refers to the organism's ability to encode, retain and recall experiences and information. Failure of memory consequently results from failure of adequately storing the memory, failure to retain the information or failure to retrieve the information later. The first and best-studied case of memory dysfunction was the 27 year old patient called H.M., who underwent a surgery for the relief of intractable epilepsy in 1953 [1]. In this surgery portions of the temporal lobe were removed bilaterally. H.M.'s seizures were better controlled after surgery, but the removal of the medial temporal lobes left him with a devastating memory deficit. In fact, H.M. showed normal short-term memory, had a good command of language and his IQ remained unchanged, but he lacked the ability to transfer new information into long-term memory $[1,2]$.

\section{TYPES OF MEMORY}

Memory formation and recall is essential for higher cognition and survival. One can differentiate between sensory, short- and long-term memories, which are defined by the duration for which a memory is expected to last. Sensory memory corresponds to sensory (visual, auditory, gustatory, olfactory or tactile) recollection 200-500 $\mu$ s after perceiving the information. In short-term memory the efficiency with which nerve impulses pass across synapses is increased for several seconds to minutes. Long-term memory requires that new synaptic connections are formed, inducing a permanent change in the neural circuit [3]. Various forms of long-term memory can furthermore be classified as either explicit (declarative) or implicit (non-declarative) and be assigned to different structures of the brain. Explicit memory requires conscious thought and can be associated with the medial temporal lobe, while implicit memory does not require conscious recollection and involves structures like the striatum, amygdala, cerebellum and the neocortex [4].

One major pathway of the brain which is primarily involved in the cortical control of emotion and in storing memory is the circuit of Papez. It plays a role in consolidating short-term to long-term memory after receiving emotional and motivational inputs from structures such as the hypothalamus, amygdala or orbital cortex. In this circuit the entorhinal cortex projects to the hippocampus, which efferents are bundled in the fornix and reach the mammillary bodies. In fact, the fornix connects the hippocampus and subcortical structures in a bi-directional manner. From the mammillary bodies, the information proceeds to the anterior nucleus of the thalamus via the mammillothalamic tract. The circuit is completed by projections of the anterior thalamic nuclei 
to the cingulate gyrus which in turn transfers the information via the cingulum to the parahippocampal gyrus of the entorhinal cortex.

The role of the Papez circuit has been the subject of many investigations designed to elucidate the neural basis of memory. As seen in the case-study of H.M. the hippocampus is essential for the consolidation of information from short-term to longterm memory, although it does not seem to store information itself [2]. In addition, structures like the hippocampus are required for spatial learning and memory.

\section{MEMORY LOSS}

Different aspects of memory are affected by damage to distinct brain regions [5]. Aging, for example, impairs hippocampal and prefrontal-cortex dependent memory and cognition. In an aging society, dementia has emerged as one of the leading health problems of our time and has been recently recognized as one of the major threats to world population [6]. Worldwide 35.6 million people suffer from dementia, a number that is anticipated to triple by 2050. The most common form of dementia is Alzheimer's Disease (AD), accounting for 60-80\% of all cases. Every year 6 million new cases of $\mathrm{AD}$ are diagnosed, imposing a tremendous burden on families and caregivers, as well as financial cost to society. To date, five drugs are approved for the treatment of AD in North America and most European countries, including four acetylcholinesterase inhibitors (Tacrine, Donepezil, Galantamine and Rivastigmine) and an N-methly-Daspartate (NMDA) receptor antagonist (Memantine) [7]. These pharmacological treatments, however, only provide limited and short-lasting symptomatic relief. In some patients substantial side-effects such as nausea and muscle weakness are seen [8].

Yet, the localization of diverse memory processes in different brain areas and the understanding of their biochemical and physiological underpinnings has opened the doors to novel therapeutic approaches, which do not require drugs. One approach, which is currently being explored, is deep brain stimulation.

\section{DEEP BRAIN STIMULATION}

Deep brain stimulation (DBS) is a reversible, minimal invasive neurosurgical intervention in the brain, aimed at treating neurological disorders. It consists of three components: the implanted pulse generator, the electrodes, and the extension. The pulse generator is a battery-powered neurostimulator, which sends electrical pulses to the brain to interfere with neural activity at the target site. The electrode is a coiled wire insulated in polyurethane with four platinum iridium contact sites and is placed in 
different nuclei of the brain. It is connected to the pulse generator by the extension, which is an insulated wire placed subcutaneously.

Worldwide more than 130,000 patients have been implanted with DBS electrodes. In fact, this procedure has become a routine second-tier therapy in patients with specific neurological disorders, including Parkinson's Disease [9], essential tremor $[10,11]$ and dystonia [12]. In patients with severe epilepsy [13], Tourette syndrome [14] and obsessive-compulsive disorder [15, 16], DBS has been shown to have good therapeutic effects. In the past years, studies have begun to explore the applicability of DBS in a widening array of psychiatric conditions including disorders of mood and cognition. DBS within the limbic system, for example, has shown positive effects in treatment-resistant depression [17-19]. Recently, DBS has been proposed as a potential tool to enhance memory functions. A first evidence for this emerged when Hamani and colleagues [20] stimulated the fornix/hypothalamus area in a patient suffering from morbid obesity. In this specific case, DBS generated detailed autobiographical memories in the patient and an electroencephalographic source localization elucidated that hypothalamic stimulation evoked activity in medial temporal lobe structures including the hippocampus. Based on this case-observation, the same group performed a phase-I trial in patients with $\mathrm{AD}[21]$. Patients with mild AD were implanted with electrodes in the area of the fornix/hypothalamus and after an intraoperative evaluation of stimulation, patients received chronic DBS for a period of 12 months. The hypothesis of this study stated that it might be possible to use DBS of the fornix to drive its activity and to modulate the circuits mediating memory functions. Indeed, memory tests confirmed possible improvements and/or slowing in the rate of cognitive decline in some patients following chronic DBS.

Despite the encouraging results of the clinical trials presented above, basic neural and chemical mechanisms underlying DBS are still debated [22]. One approach to address these issues is to investigate the effects by stimulating homologous regions in experimental animal models [23].

\section{EXPERIMENTAL DBS IN ANIMAL MODELS}

In order to investigate which neural processes are modulated by DBS or which stimulation parameters or surgical targets should be used, preclinical animal studies can be of great value [23]. The development of experimental DBS in animal models has been a major breakthrough for preclinical research. With the use of translational hardware concerning electrode compositions, preclinical studies can advance the application of clinical DBS. A DBS device can be constructed for unilateral as well as bilateral stimulation. For the latter, a bilateral electrode has to be assembled, whereas the inter- 
electrode distance depends on the brain target of stimulation. Stimulation parameters (frequency, pulse width and amplitude) can be defined according to the hypothesis. Of note, stimulation amplitudes in animal research are relatively high compared with those used in human DBS. This is because in preclinical studies, stimulation is usually acute and thus side effects are less obvious. When high amplitudes (i.e. $\geq 200 \mu \mathrm{A}$ ) are applied in chronic stimulation paradigms, substantial tissue damage can be observed [23]. Only through understanding the anatomical, physiological and neurochemical substrates of DBS, this therapy can be fine-tuned to produce the best possible symptom relief in patients.

\section{AIM OF THIS THESIS}

The overall aim of this thesis is to investigate whether DBS can be used as a tool to restore memory loss and what the mechanisms of action are.

First, state-of-the-art application of DBS in psychiatric conditions will be described (Chapter 2). Following this, I will primarily focus on dementia-related disorders and speculate on potential mechanisms of action (Chapter 3 ). In order to investigate restoration of memory loss, an experimental rat model of dementia needs to be verified. I chose for the scopolamine-induced rat model of dementia and explored effects on various brain regions (Chapter 4). Using this rat model I assessed restoration of memory loss by means of DBS in different target regions using behavioral tasks (Chapter 5 and 7). Since dementia occurs with aging, I was also engaged in a collaborative project, in which various behavioral tasks with DBS were performed in an aged animal model (Chapter 6). Finally I investigated mechanisms of action of DBS with regard to memory restoration. First, I examined the effects of DBS on dopamine levels in the VTA and substantia nigra (Chapter 8). Next, I investigated whether DBS had an effect on neural activity within certain brain regions as well as neurotransmitter release (Chapter 9). I also assessed whether DBS has any long-term effects on memory, which can be linked to neurogenic processes (Chapter 10). Finally, the main findings of this thesis will be summarized and discussed and the overall conclusion of the research will be provided (Chapter 11). 


\section{REFERENCES}

1. Scoville W, Milner B. Loss of recent memory after bilateral hippocampal lesions. J Neurol Neurosurg Psychiat. 1957;20(1):11-21.

2. Corkin S. What's new with the amnesic patient H.M.? Nature Reviews Neuroscience. 2002;3(2):1530 .

3. Davis H, Squire L. Protein synthesis and memory: A review. Psychological Bulletin. 1984;96(3):11859.

4. Graf P, Schacter D. Implicit and explicit memory for new associations in normal and amnesic subjects. Journal of Experimental Psychology: Learning, Memory, and Cognition. 1985;11:501-18.

5. Poldrack RA, Gabrieli JD. Functional anatomy of long-term memory. Journal of clinical neurophysiology : official publication of the American Electroencephalographic Society. 1997;14(4):294-310.

6. Batsch N, Mittelman M. World Alzheimer Report 2012: Overcoming the stigma of dementia. 2012.

7. Thies W, Bleiler L. 2011 Alzheimer's disease facts and figures. Alzheimer's \& dementia : the journal of the Alzheimer's Association. 2011;7(2):208-44.

8. Qaseem A, Snow V, Cross JT, Forciea MA, Hopkins R, Shekelle P, et al. Current Pharmacologic Treatment of Dementia: A Clinical Practice Guideline from the American College of Physicians and the American Academy of Family Physicians. Annals of Internal Medicine. 2008;148(5):370-8.

9. Deuschl G, Schade-Brittinger C, Krack P, Volkmann J, Schafer H, Botzel K, et al. A randomized trial of deep-brain stimulation for Parkinson's disease. The New England journal of medicine. 2006;355(9):896-908.

10. Benabid AL, Pollak P, Seigneuret E, Hoffmann D, Gay E, Perret J. Chronic VIM thalamic stimulation in Parkinson's disease, essential tremor and extra-pyramidal dyskinesias. Acta neurochirurgica Supplementum. 1993;58:39-44.

11. Hubble JP, Busenbark KL, Wilkinson S, Penn RD, Lyons K, Koller WC. Deep brain stimulation for essential tremor. Neurology. 1996;46(4):1150-3.

12. Krauss JK, Pohle T, Weber S, Ozdoba C, Burgunder JM. Bilateral stimulation of globus pallidus internus for treatment of cervical dystonia. Lancet. 1999;354(9181):837-8.

13. Fisher R, Salanova V, Witt T, Worth R, Henry T, Gross R, et al. Electrical stimulation of the anterior nucleus of thalamus for treatment of refractory epilepsy. Epilepsia. 2010;51(5):899-908.

14. Ackermans L, Duits A, van der Linden C, Tijssen M, Schruers K, Temel Y, et al. Double-blind clinical trial of thalamic stimulation in patients with Tourette syndrome. Brain : a journal of neurology. 2011;134(Pt 3):832-44.

15. Denys D, Mantione M, Figee M, van den Munckhof P, Koerselman F, Westenberg H, et al. Deep brain stimulation of the nucleus accumbens for treatment-refractory obsessive-compulsive disorder. Archives of general psychiatry. 2010;67(10):1061-8.

16. Nuttin B, Cosyns P, Demeulemeester H, Gybels J, Meyerson B. Electrical stimulation in anterior limbs of internal capsules in patients with obsessive-compulsive disorder. Lancet. 1999;354(9189):1526.

17. Bewernick BH, Hurlemann R, Matusch A, Kayser S, Grubert C, Hadrysiewicz B, et al. Nucleus accumbens deep brain stimulation decreases ratings of depression and anxiety in treatment-resistant depression. Biological psychiatry. 2010;67(2):110-6.

18. Lozano AM, Giacobbe P, Hamani C, Rizvi SJ, Kennedy SH, Kolivakis TT, et al. A multicenter pilot study of subcallosal cingulate area deep brain stimulation for treatment-resistant depression. Journal of neurosurgery. 2012;116(2):315-22. 
19. Malone DA, Jr., Dougherty DD, Rezai AR, Carpenter LL, Friehs GM, Eskandar EN, et al. Deep brain stimulation of the ventral capsule/ventral striatum for treatment-resistant depression. Biological psychiatry. 2009;65(4):267-75.

20. Hamani C, McAndrews MP, Cohn M, Oh M, Zumsteg D, Shapiro CM, et al. Memory enhancement induced by hypothalamic/fornix deep brain stimulation. Annals of Neurology. 2008;63(1):119-23.

21. Laxton AW, Tang-Wai DF, McAndrews MP, Zumsteg D, Wennberg R, Keren R, et al. A phase I trial of deep brain stimulation of memory circuits in Alzheimer's disease. Annals of Neurology. 2010;68(4):521-34.

22. Kringelbach ML, Green AL, Owen SL, Schweder PM, Aziz TZ. Sing the mind electric - principles of deep brain stimulation. Eur J Neurosci. 2010;32(7):1070-9.

23. Tan S, Vlamings R, Lim L, Sesia T, Janssen ML, Steinbusch HW, et al. Experimental deep brain stimulation in animal models. Neurosurgery. 2010;67(4):1073-9. Epub 2010/10/01. eng. 




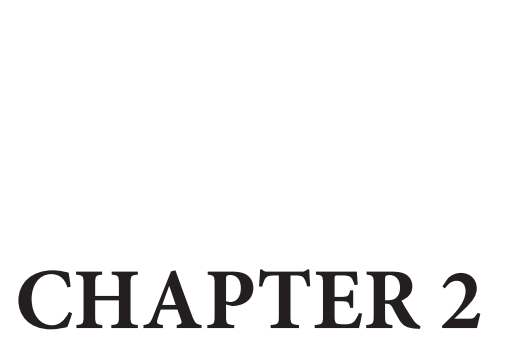

NEUROMODULATION IN PSYCHIATRIC DISORDERS

Yasin Temel, Sarah-Anna Hescham, Ali Jahanshahi, Marcus LF Janssen, Sonny KH Tan, Jacobus J van Overbeeke, Linda Ackermans, Mayke Oosterloo, Annelien Duits, Albert Leentjens and LeeWei Lim

Adapted from International Review of Neurobiology, 2012;107:283-314. 


\begin{abstract}
Psychiatric disorders are worldwide a common cause of severe and long-term disability and socio-economic burden. The management of patients with psychiatric disorders consists of drug therapy and/or psychotherapy. However, in some patients, these treatment modalities do not produce sufficient therapeutic effects or induce intolerable side-effects. For these patients neuromodulation has been suggested as a potential treatment modality. Neuromodulation includes deep brain stimulation, vagal nerve stimulation, and transcranial stimulation. The rationale for neuromodulation is derived from research identifying neurobiologically localized substrates for refractory psychiatric symptoms. Here, we review the clinical data on neuromodulation in some of the major psychiatric disorders. Relevant data from animal models will also be discussed to explain the neurobiological basis of the therapy.
\end{abstract}




\section{INTRODUCTION}

Psychiatric disorders are characterized by alterations in thinking, mood or behaviour. They cause an enormous economic burden on societies and on quality of life of individuals and families [1]. Current treatment options of the patients with psychiatric disorders include drug therapy and/or psychotherapy. However, in some patients, these treatment modalities do not produce sufficient therapeutic effects or induce intolerable side-effects. For these patients, ablative surgery and neuromodulation therapies have been proposed. In the early and mid 20th century, refractory psychiatric ("mentally ill") patients have mainly undergone ablative surgery and in some cases experimental electrical brain stimulation. The rationale for these procedures was to counteract the dysfunctional neuronal activity of specific parts of the limbic and associative brain circuits. Although this approach seems hypothesis-driven, there was only limited scientific evidence to support this view at that time. The outcome of these procedures was unfortunately not extensively documented and some patients experienced major side-effects. The latter together with advances in the field of medical ethics resulted in stopping most of the "psychosurgery" programs, except for some centres which continued.

In the late 20th century, the neurosurgical treatment of refractory psychiatric disorders underwent a revival with the application of deep brain stimulation (DBS). This technique is actually a modernized and advanced version of the previous electrical brain stimulation paradigms, also applied in human subjects [2]. Nevertheless, there are major differences between the present and past. At present, we have supporting clinical and experimental evidence, extensive medical ethical considerations and the availability of advanced stimulation technology. Recent studies have consistently shown that DBS has promising therapeutic effects in selected cases and as a result this field of Neuroscience is rapidly emerging. The rationale for DBS is related to the neurobiological mechanisms underlying psychiatric disorders, which will be explained in more detail in the sections below.

Neurobiological mechanisms underlying psychiatric disorders have been considered for a long time to depend on solitary systems such as neurotransmitters and specific cortical areas. Although these models might still explain some of the symptoms, more recent findings from both clinical and experimental studies [3] suggest a dysfunction of interrelated networks involving cortical and subcortical structures and various neuroactive substances $[4,5]$. For instance, symptoms of Tourette syndrome (TS) and obsessive-compulsive disorder (OCD) have been linked to non-motor elements of the basal ganglia-thalamocortical pathways, including the nucleus accumbens (NAc), ventral pallidum and medial parts of the thalamus, in combination with a dysfunctional mesolimbic dopaminergic system $[5,6]$. The dysfunctional network hypothesis 
provided a basis to explore DBS in psychiatric disorders. DBS is, however, not the only neuromodulation-based therapy for refractory psychiatric disorders. Others include vagal-nerve stimulation, transcranial magnetic stimulation (TMS) and transcranial direct current stimulation (tDCS). Electroconvulsive therapy, used for a long-time to treat depression for instance, is also a neuromodulation-based therapy, but is beyond the scope of this article. Here, we review the clinical data on DBS in some of the major psychiatric disorders in an alphabetic order. We will briefly discuss other neuromodulation techniques as well. Relevant data from animal models in specific sections will also be discussed to explain the neurobiological basis of the therapy.

\section{Neuromodulation in addiction}

Addiction or substance dependence is a chronic relapsing disease characterized by a strong psychological and physical dependence, resulting in a withdrawal syndrome when the use of the drug is stopped. Interestingly, like in other disorders, ablative surgery of certain brain areas, including the cingulate gyrus, the substantia innominata, ventromedial hypothalamus and the NAc have been performed to treat patients with addiction [7-12]. The rationale was to interfere with a dysfunctional neuronal circuit responsible for the symptoms. To our knowledge, no randomized controlled trials have been published thus far to test the effect of DBS on refractory addiction. Some case reports and series have been reported in which the effects of DBS of the subthalamic nucleus (STN) (Figure 1) or NAc (Figure 2) are described.

\section{Deep brain stimulation}

Recently, the effect of DBS of the NAc in three patients with alcohol addiction was described [14]. In these patients, craving fully disappeared after DBS. Two patients remained abstinent during the one year follow up, and the other reduced his alcohol consumption. In one patient also the number of cigarettes smoked per day was reduced. In this patient a hypomanic episode of two weeks was reported, which resolved after adjusting the stimulation parameters. In another publication, a case of DBS within the NAc in a patient with heroin addiction was described [15]. After surgery this patient remained abstinent from drugs during the six-year follow up. He also reduced his smoking to $75 \%$, with 10 cigarettes a day. Interestingly, the stimulator was turned off 2 to 3 years after surgery and was later removed. The improvement persisted.

In a patient with severe agoraphobia, DBS of the NAc had almost no effect on the phobia, but alcohol consumption was substantially reduced [16]. One patient who underwent DBS of the NAc for OCD decided to stop smoking 10 months after surgery, while she had many unsuccessful attempts to stop before surgery [17]. Although, the case descriptions demonstrate beneficial effects, it is still hard to draw final conclusions from these uncontrolled studies. This view is also supported when considering the vari- 

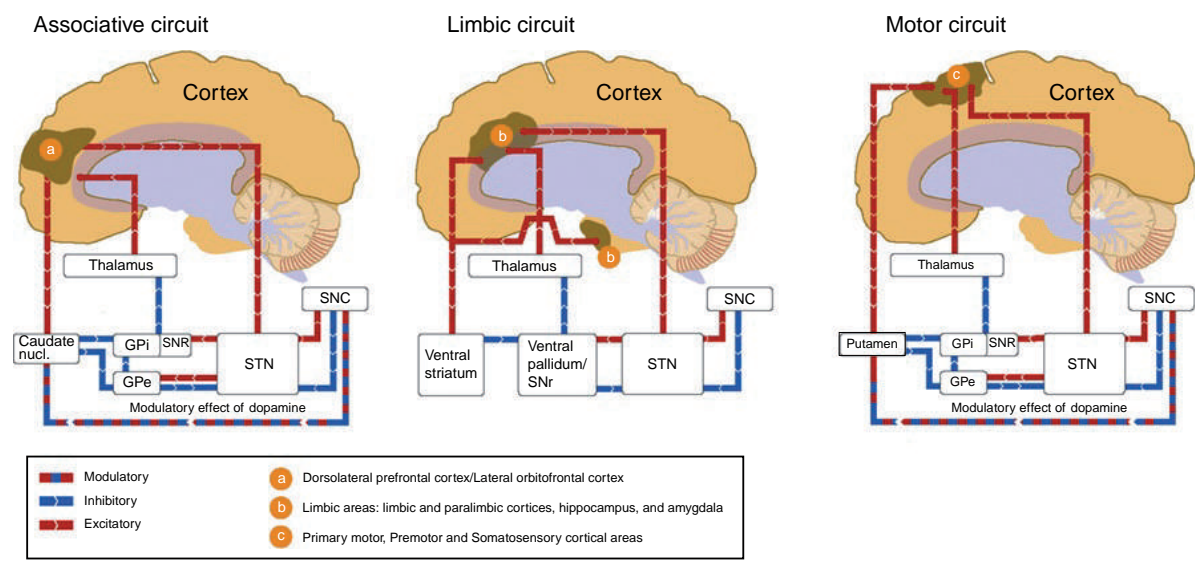

Figure 1: The subthalamic nucleus (STN) receives, integrates and projects at least three types of information: motor, limbic and associative. These are illustrated in this schematic illustration of the primate basal ganglia-thalamocortical associative, limbic and motor circuits. Associative circuit: The two associative circuits are the dorsolateral prefrontal circuit (DPC) and lateral orbitofrontal circuit (LOC), which are initiated from the dorsolateral prefrontal cortex (DLPC), and lateral orbitofrontal cortex (LOFC), respectively. The second station is the dorsolateral head and the rostrocaudal axis of the caudate nucleus. From here, this circuit is directed to the dorsomedial part of the GPi and to the rostral region of the SNr and to the anterior parts of the GPe. The GPi and SNr project to the ventroanterior (VA) and centromedian nucleus (CM) nuclei of the thalamus and the DPC is closed by the thalamocortical pathway back to the DLPC and the LOC back to the LOFC. This pathway is also known as the direct pathway. From the GPe, a projection to the STN and GPi/SNr exists. This pathway is also known as the indirect pathway. The STN is anatomically connected with both direct, through its projection to the GPi and $\mathrm{SNr}$, and indirect pathway, through its projection to the GPe. Limbic circuit: Projections from the hippocampus, the amygdala, limbic and paralimbic cortices are primarily concentrated at the level of the ventral striatum. The ventral striatum consists basically of the nucleus accumbens, ventromedial part of the caudate-putamen and the medium-celled portion of the olfactory tubercle. The ventral striatum projects in turn to the ventral pallidum (VP). From here the limbic circuit is directed to the MD nucleus of the thalamus. This circuit is closed by a thalamocortical pathway to the anterior cingulated area and medial orbitofrontal cortex. The STN has reciprocal connections with the ventral pallidum. The ventral pallidum is considered to be the major limbic circuit output region. Motor circuit: The cortical input to the motor circuit originates mainly from the primary motor, premotor and somatosensory areas. This glutamatergic input is largely directed to the putamen which projects topographically to the motor parts (ventrolateral (VL) and posterior) of the GPe and GPi, and the SNr. From the GPe, a pathway projects to the STN. The STN, mainly projects to the GPi and SNr. The SNr and the GPi serve as the output nuclei of the basal ganglia. The thalamic areas involved in the motor circuit are mainly the VL, VA and the CM. This loop is closed by means of the thalamic projection (glutamatergic) to the cortical areas. Adopted from Temel et al. [6]. 


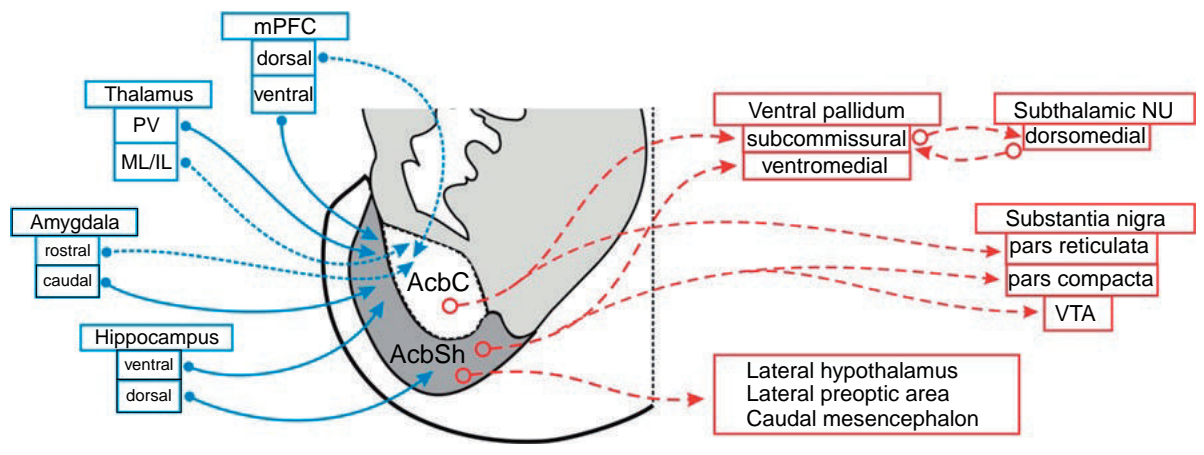

Figure 2: The nucleus accumbens is part of the ventral striatum and a popular target for DBS in several psychiatric disorders. Here we illustrate its connections with other brain areas. Schematic drawing of the inputs and outputs of the shell (AcbSh) and core (AcbC) of the nucleus accumbens. Dopaminergic, serotonergic, and noradrenergic inputs have been omitted from the drawing. Note that virtually all structures (left hand side of the scheme), although via different subdivisions or subnuclei, project to both shell and core. This also holds true for the outputs of both subdivisions (right hand side of the scheme), but it must be noted that there are also main differences. Both shell and core reach pallidal and nigral areas, albeit different parts of these basal ganglia structures. The shell, in addition projects to preoptic and hypothalamic areas and the caudal mesencephalon.

Abbreviations: ML/IL, midline and intralaminar thalamic nuclei; mPFC, medial prefrontal cortex; PV, paraventricular thalamic nucleus; VTA, ventral tegmental area. Adopted from Basar et al. [13]

able effects of NAc DBS on nicotine addiction in a case series in which only 3 out of 10 patients improved [18].

The STN is targeted for DBS in patients with advanced Parkinson's disease (PD). The STN has strong motor, associative and limbic connections (Figure 1) [6]. Interestingly, in PD patients, STN DBS resolved their addictive behaviour [19-22], however opposite effects have been found as well. In a case report, a patient without any history of addiction became a pathologic gambler after STN DBS [23]. Other studies found also varying effects of STN DBS, some patients improved, while in others the addictive behaviour remained the same or even worsened [24]. The underlying mechanisms are unclear.

\section{Transcranial stimulation}

TMS and tDCS show promising results on addictive behaviour [25-34]. Several studies have shown that TMS is effective in reducing the level of cravings for smoking, alcohol, and cocaine when applied at high frequencies to the dorsolateral prefrontal cortex [26]. TMS to the dorsal anterior cingulate cortex reduced alcohol craving and consumption as well [34]. 
In rodents, TMS increases dopamine in the dorsal hippocampus [35] and the NAc $[35,36]$. TMS in the rat has also been shown to change the expression of proteins involved in the synthesis of $\gamma$-aminobutyric acid (GABA) by two isoforms of glutamate decarboxylase [37]. The effect of TMS on addiction is not explained by local changes, but by plastic and structural changes in the neural networks involved in addiction. Animal data show that the STN is connected with the reward circuitry (ventral tegmental area and NAc) [38-41]. STN lesions or high frequency stimulation (HFS) reduces motivation to obtain cocaine while increasing the drive to attain food rewards [38, 39]. From the animal studies we can conclude that the STN plays a complex role in the reward circuit and is therefore an unpredictable target to treat addiction.

Animal studies on DBS of the NAc showed a significant reduction of drug related behaviour of either core or shell stimulation [42-45]. Also lesions of the medial NAc shell in animal models reduced addictive behaviour and decreased ethanol consumption [46].

\section{Neuromodulation in Alzheimer's disease}

Alzheimer's disease (AD) is the most frequent age-dependent form of dementia. In the early stages of $\mathrm{AD}$, the most common symptom is the inability to acquire new memories. In further progression of the disease, patients suffer from confusion, agnosia, aphasia, apraxia and finally long-term memory loss. Despite the devastating impact, there are currently no known treatments to prevent, cure or delay the progression of AD. To date only symptomatic treatments are available. Pharmacological interventions that are approved for treatment of AD in North America and most European countries include: Memantine (an NMDA receptor antagonist) for severe AD and acetylcholinesterase inhibitors for mild to moderate AD: Tacrine, Donepezil, Galantamine and Rivastigmine [47]. These pharmacological treatments, however, have limited effects which are usually short-lasting. Neuromodulation takes a completely different approach to treat AD from current medication. Researchers are currently exploring the applicability of DBS [48, 49], TMS [50-53] or tDCS $[54,55]$ to halt or reduce the progression of memory loss in $\mathrm{AD}$ and to improve the quality of life of patients and caregivers.

\section{Deep brain stimulation}

Recently, Laxton et al. [48] performed the first DBS study in which six patients with mild $\mathrm{AD}$ were implanted with electrodes in the vicinity of the fornix/hypothalamus. After an intraoperative evaluation of stimulation to survey for recollective experiences and adverse effects, patients received chronic high frequency DBS for a period of 12 months. The authors have found that the application of DBS in the hypothalamus/fornix vicinity was safe and triggered neural activity in the memory circuit, including the entorhinal and hippocampal areas. PET scans showed an early and striking reversal of 
the impaired glucose utilization in the temporal and parietal lobes that was maintained after 12 months of continuous stimulation. Evaluation of the Alzheimer's Disease Assessment Scale cognitive subscale and the Mini Mental State Examination suggested possible improvements and slowing in the progression of memory loss at 6 and 12 months, especially in patients that were less severely affected at the time of surgery. After 1 year of continuous DBS, the functional connectivity analysis demonstrated increased cerebral metabolism in cortical-subcortical and cortical-hippocampal networks. In similar cortical regions, both a higher baseline metabolism and an increase after 1 year of DBS were correlated with less decline or improvement in global cognition, memory, and quality of life [49]. When considering animal studies, DBS of the forniceal region (Figure 3), improves spatial memory performance in the object location task in a rat model of experimental dementia [56]. Memory enhancement was substantial and became comparable to the performance of controls. The current hypothesis states that this effect is accomplished by driving the fornix activity, both orthodromically as well as antidromically [56-58]. This is supported by the view that large myelinated axons produce excitatory responses upon electrical stimulation [59].

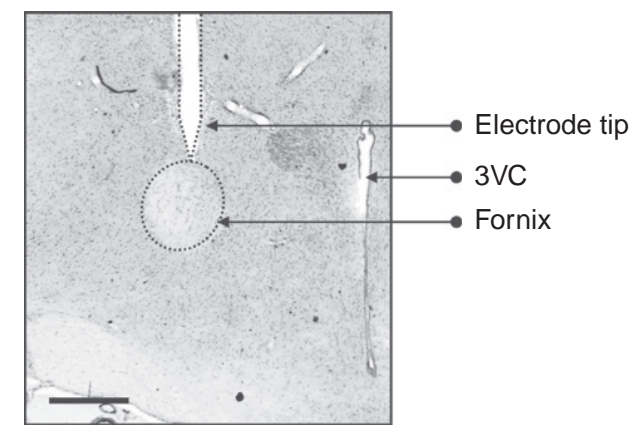

Figure 3: The fornix is part of the circuit of Papez and is considered a target for DBS in AD. Here, we illustrate the placement of the electrode in the vicinity of the rodent fornix. DBS of this area improved memory impairment in a rat model of dementia. Illustrative coronal section showing the histological verification of the electrode location in the vicinity of the fornix $(\mathrm{bar}=400 \mu \mathrm{m})$. Abbreviations: 3VC, third ventricle. Adopted from Hescham et al. [56].

\section{Transcranial stimulation}

To date, there are few reports about the effects of TMS and tDCS on memory investigating focal and non-focal neuroplasticity changes in subjects with AD [50-55]. Cotelli et al. [52] investigated the effects of TMS over the left or right dorsolateral prefrontal cortex on mild to moderate AD patients. Magnetic pulses were delivered for $600 \mathrm{~ms}$ from the onset of the stimulus presentation of a picture naming task. Repetitive TMS 
(rTMS) of both left and right dorsolateral prefrontal cortex resulted in an improvement in action naming in the mild AD group. To investigate the cumulative effects of repeated consecutive sessions and possible long-lasting effects, Cotelli and colleagues [51] assessed ten moderate AD patients before TMS treatment and at 2, 4 and 12 weeks after the onset of TMS intervention. The authors showed a significant effect of TMS on auditory sentence comprehension and language improvement. Moreover, they found long-lasting improvement in sentence comprehension eight weeks after the end of TMS treatment. Ferruci et al. [55] on the other hand, compared the effects of anodal, cathodal and sham tDCS over the temporo-parietal junction in 10 patients with mild AD. The authors found that anodal tDCS significantly improved verbal recognition memory, while cathodal tDCS significantly decreased accuracy in the word recognition task. Boggio et al. [60] also found an improvement of AD patients on an object recognition memory task during temporal and prefrontal cortex anodal tDCS, respectively.

In summary, there is initial evidence that DBS, TMS and tDCS can induce cognitive enhancement in different cognitive domains such as memory, executive functions, language, visuo-spatial abilities and attention in $\mathrm{AD}$ patients. The neurobiological basis is probably related to driving the neuronal activity of the memory circuit, which is affected by the pathology in AD. Further clinical studies are needed in order to replicate and extend the initial findings and to explore different targets of stimulation.

\section{Neuromodulation in depression}

Major depression is the most common of all psychiatric disorders and is ranked as the fourth leading cause of societal burden among all diseases. Depression can be treated effectively by either medication, and/or psychotherapy. Nevertheless, approximately $20 \%$ fail to respond to these standard therapies and nearly $60 \%$ may not achieve adequate response. For these patients, combinations of drugs and electroconvulsive therapies are often required. In patients who remain severely depressed, the so-called condition of treatment resistant depression (TRD), ablative surgeries were performed [61], similarly to the situation in other psychiatric disorders. Nowadays, this treatment modality is largely abandoned. The introduction of DBS of the subgenual cingulate gyrus [62] and later of the NAc [63] as a potential therapy in patients with TRD has redirected research in this field and neuromodulation-based therapies regained interest.

\section{Deep brain stimulation}

In 2005, Mayberg and coworkers presented their first results of DBS of the subgenual cingulate gyrus in TRD [62]. Their aim was to silence the subgenual cingulate cortex, which showed abnormal activity in chronically depressed patients. Six patients suffering from TRD received stimulating electrodes at the level of the subgenual cingulate gyrus. Striking and sustained remission of depressive symptoms was observed in four 
out of six patients. Clinically, improvement in mood was accompanied with an increase in energy, interest, psychomotor speed and a decrease of apathy and anhedonia. In addition, positron emission tomography (PET) images demonstrated normalization of the metabolic activity of the stimulated area. Recently, the same group published their long-term findings in 20 patients with TRD and confirmed the beneficial effects [64]. No significant side-effects were seen. However, two patients died by suicide during depressive relapses.

Schlaepfer and associates performed DBS of the NAc and reported beneficial effect on depressive symptoms in their three patients [63]. PET imaging showed enhanced metabolic activity in the NAc, amygdala, and dorsolateral and dorsomedial prefrontal cortices, and reduced metabolism in the ventral and ventrolateral medial prefrontal cortices. In their next two publications, the same group described more patients with a longer follow-up period $[65,66]$. In their last publication, a follow-up of up to four years for 11 patients was described. Their response criterion was at least $50 \%$ improvement in the Hamilton Depression Rating Scale (HDRS). Five out of 11 patients met the criterion of being a responder. The responders showed a strong and sustained therapeutic effect of DBS. From the non-responder group, on the other hand, one patient committed suicide and one patient attempted suicide [66].

In 2009, Malone and colleagues performed a study of DBS of the ventral capsule/ ventral striatum for TRD [67]. Fifteen patients, with TRD were included to this study. Interestingly, the response rates were $40 \%$ at 6 months, and $53.3 \%$ at the last follow-up (up to 4 years) examination. No serious adverse effects were reported. Jimenez and co-workers described the effects of DBS of the inferior thalamic peduncle region in a 49-year-old woman. The patient had a history of recurrent episodes of major depression for over 20 years and DBS resulted in substantial reduction of the depressive symptoms [68]. Kosel and associates described the case of a 62-year-old woman with TRD with comorbid neuroleptic-induced tardive dyskinesias. DBS of the globus pallidus internus (GPi) reduced not only the dyskinesias, but also the HDRS-score. The latter was reduced by circa $50 \%$ [69].

In another case report, the authors described the therapeutic effects of DBS of the lateral habenula in a patient with TRD. Interestingly, depressive symptoms reappeared when DBS was switched off as a result of an incidental bicycle accident. After turning on the stimulator, depressive symptoms were reduced substantially [70].

\section{Transcranial stimulation}

In 2008, TMS was approved by the FDA for the treatment of TRD. Recent clinical studies showed that TMS of the left dorsolateral prefrontal cortex can produce antidepressant effects [71-73], varying from moderate to substantial [73, 74]. TMS can also be used as an adjuvant therapy to enhance the effect of antidepressant drugs [75-78]. It 
is not yet clear, what the exact place of TMS and also tDCS treatment is in TRD, since DBS, a potentially more effective therapy, is gaining interest.

\section{Vagal nerve stimulation}

Vagal nerve stimulation (VNS) was first introduced as an experimental anticonvulsant procedure in dogs $[79,80]$ and few years later in patients with refractory epilepsy [81]. After it became an established therapy in refractory epilepsy, VNS was proposed as a treatment for TRD at the end of the 1990's [82, 83]. The rationale was based on the observation that epileptic patients with VNS showed an improvement of mood, without having a clear explanation for the underlying mechanism [84-86]. Studies with long-term follow up have shown that VNS can be beneficial in patients with major depression, while the therapeutic effect at short-term can be less pronounced $[83,87$, 88]. The mechanisms underlying this antidepressant effect are under investigation. Further clinical trials are needed to confirm its efficacy. See also $[89,90]$ for reviews on VNS in depression.

Data from animal and clinical studies suggest that the antidepressant effect of neuromodulation involves at least the following mechanisms; driving the neuronal activity of prefrontal limbic circuits [91], enhancing central levels of 5-hydroxytryptamine (5-HT; serotonin) $[92,93]$ and stimulating the production of neurotrophins such as brainderived neurotrophic factor (BDNF) [94-96].

\section{Neuromodulation in other psychiatric indications}

Some of the major psychiatric disorders in which neuromodulation has been applied, are described above. In case reports and case series neuromodulation-based therapies have been used to treat patients suffering from schizophrenia and attention deficit hyperactive syndrome (ADHD). These will be briefly reviewed here.

\section{Schizophrenia}

Recently, series of well-conducted clinical trials have shown that TMS applied over the left temporal regions induces clinically meaningful effects on schizophrenia [97101]. Besides TMS, DBS of hippocampus and NAc have been suggested as targets for neurosurgical intervention in schizophrenia [102], but no large well-designed clinical trials are available. Plewnia et al., has reported a case study, in which a patient with intractable OCD and residual symptoms of schizophrenia was treated with unilateral DBS of the right NAc [103]. This patient showed a substantial reduction of obsessions and compulsions as well as improvement of psychosocial functioning with DBS. 


\section{Attention deficit hyperactive syndrome}

A double-blind, sham-controlled clinical trial showed that $10 \mathrm{~Hz}$ TMS applied to the right prefrontal cortex for 10 days was effective in reducing the symptoms in a young adult with severe ADHD [104]. More recently, a double-blind randomized and controlled study demonstrated that high frequency TMS of the right prefrontal cortex improved attention in ADHD patients [105]. A significant amount of TS patients also suffer from comorbid ADHD, and DBS in these patients did not only reduce tics, but had also some beneficial effect on ADHD symptoms.

\section{CONCLUSION}

The treatment of psychiatric disorders has entered a new era with advances in neuromodulation technology. The developments in neuromodulation therapies can be summarized into two categories, non-invasive and invasive ones. The non-invasive neuromodulation therapies include mainly transcranial stimulation methods. TMS and tDCS can produce beneficial effects on refractory psychiatric symptoms on the short-term. However long-term controlled studies still need to show what the exact efficacy of the treatment modality is.

A main disadvantage seems to be that transcranial stimulation methods mainly target cortical structures and not deeply situated brain regions. For these latter ones, an invasive procedure is required, and the most frequent one is DBS. In our opinion, this is a highly promising neuromodulation therapy. Important reason for this is the availability of scientific and clinical evidence justifying the clinical use of DBS.

At present, data accumulates on the safety and efficacy of DBS, showing significant therapeutic effects in patients with refractory psychiatric disorders. Good evidence is available for the use of DBS in TS, OCD and depression. For other indications, research is still ongoing and preliminary data are encouraging. Nevertheless, a major concern is that the clinical application of DBS in psychiatric illnesses is moving faster than the scientific evidence supporting or discouraging the application of this approach. The field is no longer waiting for case reports of DBS in a new and exciting brain region, but for well-designed clinical trials backed up with robust scientific evidence. This increases the likelihood of a therapy to become successful, as illustrated by the history behind the successful application of DBS in the treatment of severe PD. Finally, we are witnessing the revival of neurosurgery in the field of psychiatric illnesses. Perhaps with a collaborative effort of neurosurgeons, psychiatrists, scientists and ethicists, the 'negative' societal reputation of psychosurgery which has originated from experiences in the 20 th century can be changed to the benefit of severely ill patients and their caregivers. 


\section{REFERENCES}

1. WorldHealthOrganization. The global burden of disease: 2004 update. World Health Organisation, 2008.

2. Bishop MP, Elder ST, Heath RG. Intracranial self-stimulation in man. Science. 1963;140(3565):394-6. eng.

3. Hamani C, Temel Y. Deep brain stimulation for psychiatric disease: contributions and validity of animal models. Sci Transl Med.4(142):142rv8. eng.

4. Nestler EJ, Barrot M, DiLeone RJ, Eisch AJ, Gold SJ, Monteggia LM. Neurobiology of depression. Neuron. 2002;34(1):13-25. Epub 2002/04/05. eng.

5. Krack P, Hariz MI, Baunez C, Guridi J, Obeso JA. Deep brain stimulation: from neurology to psychiatry? Trends Neurosci. 2010;33(10):474-84. Epub 2010/09/14. eng.

6. Temel Y, Blokland A, Steinbusch HW, Visser-Vandewalle V. The functional role of the subthalamic nucleus in cognitive and limbic circuits. Progress in neurobiology. 2005;76(6):393-413. eng.

7. Foltz EL, White LE, Jr. Pain "relief” by frontal cingulumotomy. Journal of neurosurgery. 1962;19:89100. Epub 1962/02/01. eng.

8. Balasubramaniam V, Kanaka TS, Ramanujam PB. Stereotaxic cingulumotomy for drug addiction. Neurol India. 1973;21(2):63-6. Epub 1973/06/01. eng.

9. Sharma T. Abolition of opiate hunger in humans following bilateral anterior cingulotomy. Tex Med. 1974;70(10):49-52. Epub 1974/10/01. eng.

10. Knight G. Chronic depression and drug addiction treated by stereotactic surgery. Nurs Times. 1969;65(19):583-6. Epub 1969/05/08. eng.

11. Muller D, Roeder F, Orthner H. Further results of stereotaxis in the human hypothalamus in sexual deviations. First use of this operation in addiction to drugs. Neurochirurgia (Stuttg). 1973;16(4):11326. Epub 1973/07/01. eng.

12. Gao G, Wang X, He S, Li W, Wang Q, Liang Q, et al. Clinical study for alleviating opiate drug psychological dependence by a method of ablating the nucleus accumbens with stereotactic surgery. Stereotactic and functional neurosurgery. 2003;81(1-4):96-104. Epub 2004/01/27. eng.

13. Basar K, Sesia T, Groenewegen H, Steinbusch HW, Visser-Vandewalle V, Temel Y. Nucleus accumbens and impulsivity. Prog Neurobiol. 2010;92(4):533-57. eng.

14. Muller UJ, Sturm V, Voges J, Heinze HJ, Galazky I, Heldmann M, et al. Successful treatment of chronic resistant alcoholism by deep brain stimulation of nucleus accumbens: first experience with three cases. Pharmacopsychiatry. 2009;42(6):288-91. Epub 2009/11/20. eng.

15. Zhou $\mathrm{H}, \mathrm{Xu}$ J, Jiang J. Deep brain stimulation of nucleus accumbens on heroin-seeking behaviors: a case report. Biological psychiatry. 2011;69(11):e41-2. Epub 2011/04/15. eng.

16. Kuhn J, Lenartz D, Huff W, Lee S, Koulousakis A, Klosterkoetter J, et al. Remission of alcohol dependency following deep brain stimulation of the nucleus accumbens: valuable therapeutic implications? Journal of neurology, neurosurgery, and psychiatry. 2007;78(10):1152-3. Epub 2007/09/20. eng.

17. Mantione M, van de Brink W, Schuurman PR, Denys D. Smoking cessation and weight loss after chronic deep brain stimulation of the nucleus accumbens: therapeutic and research implications: case report. Neurosurgery. 2010;66(1):E218; discussion E. Epub 2009/12/22. eng.

18. Kuhn J, Bauer R, Pohl S, Lenartz D, Huff W, Kim EH, et al. Observations on unaided smoking cessation after deep brain stimulation of the nucleus accumbens. Eur Addict Res. 2009;15(4):196-201. Epub 2009/07/23. eng. 
19. Ardouin C, Voon V, Worbe Y, Abouazar N, Czernecki V, Hosseini H, et al. Pathological gambling in Parkinson's disease improves on chronic subthalamic nucleus stimulation. Movement disorders : official journal of the Movement Disorder Society. 2006;21(11):1941-6. Epub 2006/09/15. eng.

20. Bandini F, Primavera A, Pizzorno M, Cocito L. Using STN DBS and medication reduction as a strategy to treat pathological gambling in Parkinson's disease. Parkinsonism \& related disorders. 2007;13(6):369-71. Epub 2006/10/20. eng.

21. Witjas T, Baunez C, Henry JM, Delfini M, Regis J, Cherif AA, et al. Addiction in Parkinson's disease: impact of subthalamic nucleus deep brain stimulation. Movement disorders : official journal of the Movement Disorder Society. 2005;20(8):1052-5. Epub 2005/04/29. eng.

22. Knobel D, Aybek S, Pollo C, Vingerhoets FJ, Berney A. Rapid resolution of dopamine dysregulation syndrome (DDS) after subthalamic DBS for Parkinson disease (PD): a case report. Cogn Behav Neurol. 2008;21(3):187-9. Epub 2008/09/18. eng.

23. Smeding HM, Goudriaan AE, Foncke EM, Schuurman PR, Speelman JD, Schmand B. Pathological gambling after bilateral subthalamic nucleus stimulation in Parkinson disease. Journal of neurology, neurosurgery, and psychiatry. 2007;78(5):517-9. Epub 2007/01/11. eng.

24. Lim SY, O'Sullivan SS, Kotschet K, Gallagher DA, Lacey C, Lawrence AD, et al. Dopamine dysregulation syndrome, impulse control disorders and punding after deep brain stimulation surgery for Parkinson's disease. Journal of clinical neuroscience : official journal of the Neurosurgical Society of Australasia. 2009;16(9):1148-52. Epub 2009/06/26. eng.

25. Wing VC, Bacher I, Wu BS, Daskalakis ZJ, George TP. High frequency repetitive transcranial magnetic stimulation reduces tobacco craving in schizophrenia. Schizophr Res. 2012. Epub 2012/04/03. Eng.

26. Barr MS, Farzan F, Wing VC, George TP, Fitzgerald PB, Daskalakis ZJ. Repetitive transcranial magnetic stimulation and drug addiction. Int Rev Psychiatry. 2011;23(5):454-66. Epub 2011/12/28. eng.

27. Camprodon JA, Martinez-Raga J, Alonso-Alonso M, Shih MC, Pascual-Leone A. One session of high frequency repetitive transcranial magnetic stimulation (rTMS) to the right prefrontal cortex transiently reduces cocaine craving. Drug Alcohol Depend. 2007;86(1):91-4. Epub 2006/09/15. eng.

28. Eichhammer P, Johann M, Kharraz A, Binder H, Pittrow D, Wodarz N, et al. High-frequency repetitive transcranial magnetic stimulation decreases cigarette smoking. The Journal of clinical psychiatry. 2003;64(8):951-3. Epub 2003/08/21. eng.

29. Fecteau S, Knoch D, Fregni F, Sultani N, Boggio P, Pascual-Leone A. Diminishing risk-taking behavior by modulating activity in the prefrontal cortex: a direct current stimulation study. The Journal of neuroscience : the official journal of the Society for Neuroscience. 2007;27(46):12500-5. Epub 2007/11/16. eng.

30. Amiaz R, Levy D, Vainiger D, Grunhaus L, Zangen A. Repeated high-frequency transcranial magnetic stimulation over the dorsolateral prefrontal cortex reduces cigarette craving and consumption. Addiction (Abingdon, England). 2009;104(4):653-60. English.

31. Johann M, Wiegand R, Kharraz A, Bobbe G, Sommer G, Hajak G, et al. [Transcranial magnetic stimulation for nicotine dependence]. Transkranielle Magnetstimulation bei Nikotinabhangigkeit. Psychiatr Prax. 2003;30 Suppl 2:S129-31. German.

32. Mishra BR, Nizamie SH, Das B, Praharaj SK. Efficacy of repetitive transcranial magnetic stimulation in alcohol dependence: a sham-controlled study. Addiction (Abingdon, England). 2010;105(1):49-55. English.

33. Politi E, Fauci E, Santoro A, Smeraldi E. Daily sessions of transcranial magnetic stimulation to the left prefrontal cortex gradually reduce cocaine craving. Am J Addict. 2008;17(4):345-6. English. 
34. De Ridder D, Vanneste S, Kovacs S, Sunaert S, Dom G. Transient alcohol craving suppression by rTMS of dorsal anterior cingulate: an fMRI and LORETA EEG study. Neuroscience letters. 2011;496(1):510. Epub 2011/04/05. eng.

35. Keck ME, Welt T, Muller MB, Erhardt A, Ohl F, Toschi N, et al. Repetitive transcranial magnetic stimulation increases the release of dopamine in the mesolimbic and mesostriatal system. Neuropharmacology. 2002;43(1):101-9. Epub 2002/09/06. eng.

36. Erhardt A, Sillaber I, Welt T, Muller MB, Singewald N, Keck ME. Repetitive transcranial magnetic stimulation increases the release of dopamine in the nucleus accumbens shell of morphine-sensitized rats during abstinence. Neuropsychopharmacology : official publication of the American College of Neuropsychopharmacology. 2004;29(11):2074-80. Epub 2004/06/10. eng.

37. Trippe J, Mix A, Aydin-Abidin S, Funke K, Benali A. theta burst and conventional low-frequency rTMS differentially affect GABAergic neurotransmission in the rat cortex. Experimental brain research Experimentelle Hirnforschung Experimentation cerebrale. 2009;199(3-4):411-21. Epub 2009/08/25. eng.

38. Rouaud T, Lardeux S, Panayotis N, Paleressompoulle D, Cador M, Baunez C. Reducing the desire for cocaine with subthalamic nucleus deep brain stimulation. Proceedings of the National Academy of Sciences of the United States of America. 2010;107(3):1196-200. Epub 2010/01/19. eng.

39. Baunez C, Dias C, Cador M, Amalric M. The subthalamic nucleus exerts opposite control on cocaine and 'natural' rewards. Nature neuroscience. 2005;8(4):484-9. eng.

40. Uslaner JM, Yang P, Robinson TE. Subthalamic nucleus lesions enhance the psychomotor-activating, incentive motivational, and neurobiological effects of cocaine. The Journal of neuroscience : the official journal of the Society for Neuroscience. 2005;25(37):8407-15. Epub 2005/09/16. eng.

41. Lardeux S, Baunez C. Alcohol preference influences the subthalamic nucleus control on motivation for alcohol in rats. Neuropsychopharmacology : official publication of the American College of Neuropsychopharmacology. 2008;33(3):634-42. Epub 2007/04/27. eng.

42. Liu HY, Jin J, Tang JS, Sun WX, Jia H, Yang XP, et al. Chronic deep brain stimulation in the rat nucleus accumbens and its effect on morphine reinforcement. Addict Biol. 2008;13(1):40-6. Epub 2008/02/14. eng.

43. Knapp CM, Tozier L, Pak A, Ciraulo DA, Kornetsky C. Deep brain stimulation of the nucleus accumbens reduces ethanol consumption in rats. Pharmacol Biochem Behav. 2009;92(3):474-9. Epub 2009/05/26. eng.

44. Vassoler FM, Schmidt HD, Gerard ME, Famous KR, Ciraulo DA, Kornetsky C, et al. Deep brain stimulation of the nucleus accumbens shell attenuates cocaine priming-induced reinstatement of drug seeking in rats. The Journal of neuroscience : the official journal of the Society for Neuroscience. 2008;28(35):8735-9. Epub 2008/08/30. eng.

45. Henderson MB, Green AI, Bradford PS, Chau DT, Roberts DW, Leiter JC. Deep brain stimulation of the nucleus accumbens reduces alcohol intake in alcohol-preferring rats. Neurosurg Focus. 2010;29(2):E12. Epub 2010/08/03. eng.

46. Dhaher R, Finn DA, Oberbeck DL, Yoneyama N, Snelling CC, Wu W, et al. Electrolytic lesions of the medial nucleus accumbens shell selectively decrease ethanol consumption without altering preference in a limited access procedure in C57BL/6J mice. Pharmacol Biochem Behav. 2009;92(2):335-42. Epub 2009/04/09. eng.

47. Thies W, Bleiler L. 2011 Alzheimer's disease facts and figures. Alzheimer's \& dementia : the journal of the Alzheimer's Association. 2011;7(2):208-44. 
48. Laxton AW, Tang-Wai DF, McAndrews MP, Zumsteg D, Wennberg R, Keren R, et al. A phase I trial of deep brain stimulation of memory circuits in Alzheimer's disease. Annals of Neurology. 2010;68(4):521-34.

49. Smith GS, Laxton AW, Tang-Wai DF, McAndrews MP, Diaconescu AO, Workman CI, et al. Increased Cerebral Metabolism After 1 Year of Deep Brain Stimulation in Alzheimer DiseaseIncreased Cerebral Metabolism After 1 Year of DBS. Arch Neurol. 2012:1-8.

50. Bentwich J, Dobronevsky E, Aichenbaum S, Shorer R, Peretz R, Khaigrekht M, et al. Beneficial effect of repetitive transcranial magnetic stimulation combined with cognitive training for the treatment of Alzheimer's disease: a proof of concept study. Journal of Neural Transmission. 2011;118(3):463-71.

51. Cotelli M, Calabria M, Manenti R, Rosini S, Zanetti O, Cappa SF, et al. Improved language performance in Alzheimer disease following brain stimulation. Journal of Neurology, Neurosurgery \& Psychiatry. 2011;82(7):794-7.

52. Cotelli M, Manenti R, Cappa SF, Geroldi C, Zanetti O, Rossini PM, et al. Effect of Transcranial Magnetic Stimulation on Action Naming in Patients With Alzheimer Disease. Arch Neurol. 2006;63(11):1602-4.

53. Cotelli M, Manenti R, Cappa SF, Zanetti O, Miniussi C. Transcranial magnetic stimulation improves naming in Alzheimer disease patients at different stages of cognitive decline. European Journal of Neurology. 2008;15(12):1286-92.

54. Boggio PS, Khoury LP, Martins DCS, Martins OEMS, de Macedo EC, Fregni F. Temporal cortex direct current stimulation enhances performance on a visual recognition memory task in Alzheimer disease. Journal of Neurology, Neurosurgery \& Psychiatry. 2009;80(4):444-7.

55. Ferrucci R, Mameliç F, Guidi I, Mrakic-Sposta S, Vergari M, Marceglia S, et al. Transcranial direct current stimulation improves recognition memory in Alzheimer disease. Neurology. 2008;71(7):4938.

56. Hescham S, Lim LW, Jahanshahi A, Steinbusch HW, Prickaerts J, Blokland A, et al. Deep brain stimulation of the forniceal area enhances memory functions in experimental dementia: The role of stimulation parameters. Brain Stimul 2012;[Epub ahead of print].

57. Hamani C, Dubiela FP, Soares JCK, Shin D, Bittencourt S, Covolan L, et al. Anterior thalamus deep brain stimulation at high current impairs memory in rats. Exp Neurol. 2010;225(1):154-62.

58. Hamani C, McAndrews MP, Cohn M, Oh M, Zumsteg D, Shapiro CM, et al. Memory enhancement induced by hypothalamic/fornix deep brain stimulation. Annals of Neurology. 2008;63(1):119-23.

59. Ranck JB. Which elements are excited in electrical stimulation of mammalian central nervous system: A review. Brain Res. 1975;98(3):417-40.

60. Boggio PS, Fregni F, Valasek C, Ellwood S, Chi R, Gallate J, et al. Temporal Lobe Cortical Electrical Stimulation during the Encoding and Retrieval Phase Reduces False Memories. PLoS ONE. 2009;4(3):e4959.

61. Andrade P, Noblesse LH, Temel Y, Ackermans L, Lim LW, Steinbusch HW, et al. Neurostimulatory and ablative treatment options in major depressive disorder: a systematic review. Acta neurochirurgica.152(4):565-77. eng.

62. Mayberg HS, Lozano AM, Voon V, McNeely HE, Seminowicz D, Hamani C, et al. Deep brain stimulation for treatment-resistant depression. Neuron. 2005;45(5):651-60.

63. Schlaepfer TE, Cohen MX, Frick C, Kosel M, Brodesser D, Axmacher N, et al. Deep Brain Stimulation to Reward Circuitry Alleviates Anhedonia in Refractory Major Depression. Neuropsychopharmacology. 2007. 
64. Kennedy SH, Giacobbe P, Rizvi SJ, Placenza FM, Nishikawa Y, Mayberg HS, et al. Deep brain stimulation for treatment-resistant depression: follow-up after 3 to 6 years. Am J Psychiatry.168(5):502-10. Epub 2011/02/03. eng.

65. Bewernick BH, Hurlemann R, Matusch A, Kayser S, Grubert C, Hadrysiewicz B, et al. Nucleus accumbens deep brain stimulation decreases ratings of depression and anxiety in treatment-resistant depression. Biol Psychiatry.67(2):110-6. eng.

66. Bewernick BH, Kayser S, Sturm V, Schlaepfer TE. Long-Term Effects of Nucleus Accumbens Deep Brain Stimulation in Treatment-Resistant Depression: Evidence for Sustained Efficacy. Neuropsychopharmacology. Eng.

67. Malone DA, Jr., Dougherty DD, Rezai AR, Carpenter LL, Friehs GM, Eskandar EN, et al. Deep brain stimulation of the ventral capsule/ventral striatum for treatment-resistant depression. Biological psychiatry. 2009;65(4):267-75

68. Jimenez F, Velasco F, Salin-Pascual R, Hernandez JA, Velasco M, Criales JL, et al. A patient with a resistant major depression disorder treated with deep brain stimulation in the inferior thalamic peduncle. Neurosurgery. 2005;57(3):585-93; discussion -93. Epub 2005/09/08. eng.

69. Kosel M, Sturm V, Frick C, Lenartz D, Zeidler G, Brodesser D, et al. Mood improvement after deep brain stimulation of the internal globus pallidus for tardive dyskinesia in a patient suffering from major depression. J Psychiatr Res. 2007;41(9):801-3. Epub 2006/09/12. eng.

70. Sartorius A, Kiening KL, Kirsch P, von Gall CC, Haberkorn U, Unterberg AW, et al. Remission of major depression under deep brain stimulation of the lateral habenula in a therapy-refractory patient. Biol Psychiatry.67(2):e9-e11. Epub 2009/10/23. eng.

71. Schutter DJ. Antidepressant efficacy of high-frequency transcranial magnetic stimulation over the left dorsolateral prefrontal cortex in double-blind sham-controlled designs: a meta-analysis. Psychol Med. 2009;39(1):65-75. Epub 2008/05/02. eng.

72. Couturier JL. Efficacy of rapid-rate repetitive transcranial magnetic stimulation in the treatment of depression: a systematic review and meta-analysis. J Psychiatry Neurosci. 2005;30(2):83-90. Epub 2005/03/31. eng.

73. Martin JL, Barbanoj MJ, Schlaepfer TE, Thompson E, Perez V, Kulisevsky J. Repetitive transcranial magnetic stimulation for the treatment of depression. Systematic review and meta-analysis. $\mathrm{Br} \mathrm{J}$ Psychiatry. 2003;182:480-91. Epub 2003/06/05. eng.

74. Kozel FA, George MS. Meta-analysis of left prefrontal repetitive transcranial magnetic stimulation (rTMS) to treat depression. J Psychiatr Pract. 2002;8(5):270-5. Epub 2005/06/30. eng.

75. Rossini D, Magri L, Lucca A, Giordani S, Smeraldi E, Zanardi R. Does rTMS hasten the response to escitalopram, sertraline, or venlafaxine in patients with major depressive disorder? A double-blind, randomized, sham-controlled trial. J Clin Psychiatry. 2005;66(12):1569-75. Epub 2006/01/13. eng.

76. Rumi DO, Gattaz WF, Rigonatti SP, Rosa MA, Fregni F, Rosa MO, et al. Transcranial magnetic stimulation accelerates the antidepressant effect of amitriptyline in severe depression: a double-blind placebo-controlled study. Biol Psychiatry. 2005;57(2):162-6. Epub 2005/01/18. eng.

77. Bretlau LG, Lunde M, Lindberg L, Unden M, Dissing S, Bech P. Repetitive transcranial magnetic stimulation (rTMS) in combination with escitalopram in patients with treatment-resistant major depression: a double-blind, randomised, sham-controlled trial. Pharmacopsychiatry. 2008;41(2):417. Epub 2008/03/04. eng.

78. Herwig U, Fallgatter AJ, Hoppner J, Eschweiler GW, Kron M, Hajak G, et al. Antidepressant effects of augmentative transcranial magnetic stimulation: randomised multicentre trial. Br J Psychiatry. 2007;191:441-8. Epub 2007/11/06. eng. 
79. Zabara J. Inhibition of experimental seizures in canines by repetitive vagal stimulation. Epilepsia. 1992;33(6):1005-12. Epub 1992/11/01. eng.

80. Zabara J. Peripheral control of hypersynchronous discharge in epilepsy. . Electroencephalogr Clin Neurophysiol. 1985;61(3):S162.

81. Penry JK, Dean JC. Prevention of intractable partial seizures by intermittent vagal stimulatioin in humans: preliminary results. Epilepsia. 1990;31(Suppl 2):S40-S3.

82. George MS, Sackeim HA, Rush AJ, Marangell LB, Nahas Z, Husain MM, et al. Vagus nerve stimulation: a new tool for brain research and therapy. Biol Psychiatry. 2000;47(4):287-95. Epub 2000/02/25. eng.

83. Rush AJ, George MS, Sackeim HA, Marangell LB, Husain MM, Giller C, et al. Vagus nerve stimulation (VNS) for treatment-resistant depressions: a multicenter study. Biol Psychiatry. 2000;47(4):276-86. Epub 2000/02/25. eng.

84. Harden CL, Pulver MC, Ravdin LD, Nikolov B, Halper JP, Labar DR. A Pilot Study of Mood in Epilepsy Patients Treated with Vagus Nerve Stimulation. Epilepsy Behav. 2000;1(2):93-9. Epub 2003/03/01. Eng.

85. Hoppe C, Helmstaedter C, Scherrmann J, Elger CE. Self-Reported Mood Changes following 6 Months of Vagus Nerve Stimulation in Epilepsy Patients. Epilepsy Behav. 2001;2(4):335-42. Epub 2003/03/01. eng.

86. Elger G, Hoppe C, Falkai P, Rush AJ, Elger CE. Vagus nerve stimulation is associated with mood improvements in epilepsy patients. Epilepsy Res. 2000;42(2-3):203-10. Epub 2000/11/14. eng.

87. Rush AJ, Sackeim HA, Marangell LB, George MS, Brannan SK, Davis SM, et al. Effects of 12 months of vagus nerve stimulation in treatment-resistant depression: a naturalistic study. Biol Psychiatry. 2005;58(5):355-63. Epub 2005/09/06. eng.

88. Schlaepfer TE, Frick C, Zobel A, Maier W, Heuser I, Bajbouj M, et al. Vagus nerve stimulation for depression: efficacy and safety in a European study. Psychol Med. 2008;38(5):651-61. Epub 2008/01/08. eng.

89. Milby AH, Halpern CH, Baltuch GH. Vagus nerve stimulation for epilepsy and depression. Neurotherapeutics. 2008;5(1):75-85. eng.

90. Park MC, Goldman MA, Carpenter LL, Price LH, Friehs GM. Vagus nerve stimulation for depression: rationale, anatomical and physiological basis of efficacy and future prospects. Acta Neurochir Suppl. 2007;97(Pt 2):407-16. eng.

91. Ressler KJ, Mayberg HS. Targeting abnormal neural circuits in mood and anxiety disorders: from the laboratory to the clinic. Nature neuroscience. 2007;10(9):1116-24. eng.

92. Hamani C, Diwan M, Macedo CE, Brandao ML, Shumake J, Gonzalez-Lima F, et al. Antidepressantlike effects of medial prefrontal cortex deep brain stimulation in rats. Biol Psychiatry. 2010;67(2):11724.

93. Hamani C, Machado DC, Hipolide DC, Dubiela FP, Suchecki D, Macedo CE, et al. Deep Brain Stimulation Reverses Anhedonic-Like Behavior in a Chronic Model of Depression: Role of Serotonin and BDNF. Biol Psychiatry. 2011;in press.

94. Shimizu E, Hashimoto K, Okamura N, Koike K, Komatsu N, Kumakiri C, et al. Alterations of serum levels of brain-derived neurotrophic factor (BDNF) in depressed patients with or without antidepressants. Biol Psychiatry. 2003;54(1):70-5. Epub 2003/07/05. eng.

95. Smith MA, Makino S, Kvetnansky R, Post RM. Stress and glucocorticoids affect the expression of brain-derived neurotrophic factor and neurotrophin-3 mRNAs in the hippocampus. J Neurosci. 1995;15(3 Pt 1):1768-77. Epub 1995/03/01. eng. 
96. Nibuya M, Morinobu S, Duman RS. Regulation of BDNF and trkB mRNA in rat brain by chronic electroconvulsive seizure and antidepressant drug treatments. J Neurosci. 1995;15(11):7539-47. Epub 1995/11/01. eng.

97. Hoffman RE, Gueorguieva R, Hawkins KA, Varanko M, Boutros NN, Wu YT, et al. Temporoparietal transcranial magnetic stimulation for auditory hallucinations: safety, efficacy and moderators in a fifty patient sample. Biol Psychiatry. 2005;58(2):97-104. Epub 2005/06/07. eng.

98. Hoffman RE, Hawkins KA, Gueorguieva R, Boutros NN, Rachid F, Carroll K, et al. Transcranial magnetic stimulation of left temporoparietal cortex and medication-resistant auditory hallucinations. Arch Gen Psychiatry. 2003;60(1):49-56. Epub 2003/01/07. eng.

99. Fitzgerald PB, Benitez J, Daskalakis JZ, De Castella A, Kulkarni J. The treatment of recurring auditory hallucinations in schizophrenia with rTMS. World J Biol Psychiatry. 2006;7(2):119-22. Epub 2006/05/11. eng.

100. Jardri R, Lucas B, Delevoye-Turrell Y, Delmaire C, Delion P, Thomas P, et al. An 11-year-old boy with drug-resistant schizophrenia treated with temporo-parietal rTMS. Mol Psychiatry. 2007;12(4):320. Epub 2007/03/29. eng.

101. Jin Y, Potkin SG, Kemp AS, Huerta ST, Alva G, Thai TM, et al. Therapeutic effects of individualized alpha frequency transcranial magnetic stimulation (alphaTMS) on the negative symptoms of schizophrenia. Schizophr Bull. 2006;32(3):556-61. Epub 2005/10/29. eng.

102. Mikell CB, McKhann GM, Segal S, McGovern RA, Wallenstein MB, Moore H. The hippocampus and nucleus accumbens as potential therapeutic targets for neurosurgical intervention in schizophrenia. Stereotact Funct Neurosurg. 2009;87(4):256-65. Epub 2009/06/27. eng.

103. Plewnia C, Schober F, Rilk A, Buchkremer G, Reimold M, Wachter T, et al. Sustained improvement of obsessive-compulsive disorder by deep brain stimulation in a woman with residual schizophrenia. Int J Neuropsychopharmacol. 2008;11(8):1181-3. Epub 2008/08/14. eng.

104. Weaver L, Rostain AL, Mace W, Akhtar U, Moss E, O’Reardon JP. Transcranial magnetic stimulation (TMS) in the treatment of attention-deficit/hyperactivity disorder in adolescents and young adults: a pilot study. J Ect. 2012;28(2):98-103. Epub 2012/05/04. eng.

105. Bloch Y, Harel EV, Aviram S, Govezensky J, Ratzoni G, Levkovitz Y. Positive effects of repetitive transcranial magnetic stimulation on attention in ADHD Subjects: a randomized controlled pilot study. World J Biol Psychiatry. 2010;11(5):755-8. Epub 2010/06/05. eng. 



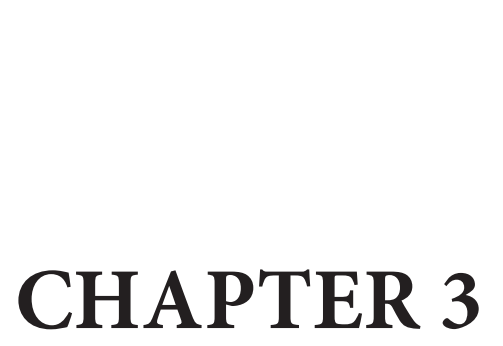

DEEP BRAIN STIMULATION IN DEMENTIARELATED DISORDERS

Sarah Hescham, LeeWei Lim, Ali Jahanshahi, Arjan Blokland, Yasin Temel

Neuroscience and Biobehavioral Reviews, 2013; 37(10 Pt 2):2666-75. 


\section{ABSTRACT}

Memory loss is the key symptom of dementia-related disorders, including the prevalent Alzheimer's disease (AD). To date, pharmacological treatments for $\mathrm{AD}$ have limited and short-lasting effects. Therefore, researchers are investigating novel therapies such as deep brain stimulation (DBS) to halt or reduce the progression of memory impairment. Clinical and preclinical studies have been performed and stimulations of the fornix, entorhinal cortex and nucleus basalis of Meynert have been carried out. The results of these studies suggest that DBS has the potential to enhance memory functions in patients and animal models. The mechanisms underlying memory enhancement may include the release of specific neurotransmitters and neuroplasticity. Some authors suggest that DBS might even be disease-modifying. Nevertheless, it is still premature to conclude that DBS can be used in the treatment of $\mathrm{AD}$, and the field will wait for the results of ongoing clinical trials. 


\section{INTRODUCTION}

Dementia is the condition of severely impaired cognitive functioning in various domains and has a substantial negative effect on patients, families and caregivers. There are different types of dementia, amongst others Alzheimer's disease, vascular dementia, Parkinson's disease dementia, Huntington's disease, alcohol-related dementia and Creutzfeldt-Jakob disease. The most prevalent cause of dementia is Alzheimer's disease (AD), which accounts for an estimated $50-80 \%$ of all cases. AD is a progressive neurodegenerative disease, which has a detrimental impact on the quality of life of patients. The age-standardized prevalence of people aged 65 years or older of population-based studies in Europe suggests that $4.4 \%$ suffer from $\mathrm{AD}$ [1]. In the United States, the study of a national representative sample of people aged more than 70 years provided a prevalence for $\mathrm{AD}$ of $9.7 \%$ [2]. In the early-stage of $\mathrm{AD}$, cognition and the ability to acquire new memories are impaired. In later stages, symptoms include progressive cognitive deterioration, long-term memory loss, aphasia, apraxia and finally the inability to perform activities of daily living. Also behavioural and psychological symptoms, i.e. agitation, depression and aggressive behaviour, occur. Structural and functional imaging studies have shown a generalised cerebral atrophy and a fluordesoxyglucose - positron emission tomography (FDG-PET) measured glucose hypometabolism in $\mathrm{AD}$ patients, which is often more focused on the frontal regions, the medial temporal lobe and the parietal regions [3]. The dysfunction and death of neurons is associated with cytoskeletal abnormalities, such as neurofibrillary tangles, as well as amyloid plaques $[4,5]$. The mean life expectancy following diagnosis is approximately seven years [6]. Currently only symptomatic treatments are available for AD. There are no known treatments that cure or delay the progression of this neurodegenerative disease. Pharmacological therapies that are approved for treatment of AD in North America and most European countries include memantine (an N-methyl-D-aspartate receptor antagonist) for severe $\mathrm{AD}$ and few acetylcholinesterase inhibitors for mild to moderate AD such as tacrine, donepezil, galantamine and rivastigmine [4].

These pharmacological treatments, however, are not effective for every patient and only improve symptoms temporarily. In some patients substantial side-effects such as gastrointestinal symptoms (nausea, vomiting, diarrhea), eating disorder/weight loss, dizziness and muscle cramps are seen [7]. Therefore, researchers are currently exploring the applicability of novel non-drug based therapies, such as deep brain stimulation (DBS) [8-10], transcranial magnetic stimulation (TMS) or transcranial direct current stimulation (tDCS) [11] to reduce or halt the progression of memory loss in AD and ultimately to improve the quality of life of patients and their caregivers.

Deep brain stimulation (DBS) is a minimal invasive surgical treatment involving the implantation of electrodes, which deliver electrical impulses to specific parts of 
the brain. It has been shown that DBS has substantial therapeutic effects in a range of neurological disorders, including Parkinson's disease, Tourette's syndrome and severe forms of epilepsy [12-16]. In the past years, the applicability of DBS in psychiatry has been evaluated in affective disorders. DBS of 'key' regions within the limbic system resulted in therapeutic effects in patients with treatment-resistant depression [17-19] and obsessive-compulsive disorder [20]. In this respect, recent clinical $[9,10]$ and preclinical $[8,21,22]$ studies have suggested that DBS can be used as a tool to enhance memory functions. TMS and tDCS, on the other hand, are non-invasive techniques that can induce significant and long-lasting changes in cognitive function in both healthy volunteers and patients with neurological disease [23-26]. To date, there are few reports about the effects of rTMS and tDCS on memory. Most of them investigate focal and non-focal neuroplasticity changes in subjects with mild AD disease [27-29]. For a detailed review on TMS and tDCS on Alzheimer's disease see Boggio et al. [11]. Here, we will focus on DBS and address the question whether there is a place for DBS as a treatment of memory-related disorders. We will review relevant preclinical and clinical literature.

\section{OUTLINE OF THE REVIEW}

This review was based on articles identified by a PubMed search with the terms "Deep brain stimulation", "dementia" and "memory" as the main keywords. Relevant articles were also identified from the reference lists of articles, review papers, and book chapters. Only original data is described in this review, giving preference to behavioral studies investigating memory performance of subjects. Review papers were utilized for the explanation of DBS mechanisms.

The outline of the review is as follows: in the first section, the neuroanatomical circuit responsible for memory functions is summarized in order to provide background information for the choice of the different targets for DBS. In the second section, preclinical studies, experimental findings in humans and clinical studies which have applied DBS to modulate memory functions are outlined. Finally, we provide an overall discussion of the evidence available thus far.

\section{The memory circuit}

The selection of the brain regions for DBS is mainly based on the so-called memory circuit of the brain. The major pathway for memory, including long-term storage and recognition memory, is located in the medial temporal lobe (i.e. hippocampus, rhinal cortices and amygdala) and diencephalic structures (i.e. mammillary bodies, thalamus). In the classical memory circuit, the entorhinal cortex projects to the hippocampus via 
the perforant pathway. The perforant pathway is considered the main afferent pathway to the hippocampus, where glutamatergic fibers from the entorhinal area reach the granule cell layer of the dentate gyrus. Moreover, the perforant pathway also projects to the subiculum as well as to the CA3 and CA1 subfield of the hippocampus [30]. From the dentate gyrus connections are made to the pyramidal neurons in the CA3 subfield via mossy fibers. Lastly, CA3 neurons project to pyramidal neurons in the CA1 through Schaffer collaterals. It is known that pyramidal neurons contain glutamatergic and GABAergic synapses [31].

From the hippocampus the information proceeds through the subiculum to the fimbria and the fornix. The precommissural branch of the fornix projects amongst others to the anterior cingulate cortex via the septal nuclei and ventral striatum. Cholinergic fibers from the basal forebrain, including the septal nuclei and the nucleus basalis of Meynert (NBM), run through the fornix. Some fibers from the fornix also pass through the anterior commissure to the contralateral hippocampus. The postcommissural branch of the fornix projects to the anterior nuclei of the thalamus and the mammillary bodies. Because the mammillothalamic tract couples the mammillary bodies and the anterior thalamic nucleus, the hippocampus can have a direct as well as indirect effect on the thalamus $[32,33]$. Findings from several electrophysiological studies indicate that the anterior nucleus of the thalamus is the primary source of glutamatergic input to cingulate neurons $[34,35]$. Thus, the postcommissural branch of the fornix reaches the cingulate gyrus through the anterior thalamic nucleus. This memory circuit is completed by projections of the cingulate gyrus to the entorhinal cortex of the parahippocampal region.

Evolving experimental approaches have contributed to a nuanced view of understanding the different structures of the memory circuitry, since lesions in the memory circuit can mimic typical memory deficits seen in ageing and dementia. Hippocampal and anterior thalamic nuclei lesions predominantly affect episodic [36, 37] and spatial memory $[38,39]$. To some extent also recognition memory can be attributed to these structures [40-43]. Fornix, cingulum and mammillary bodies, on the other hand, can be assigned merely to spatial memory functions [44-48], and memory recall [49]. Finally, the parahippocampal gyrus and entorhinal cortex are involved in both, spatial [50-53] and recognition memory $[54,55]$. There is evidence that glutamatergic and GABAergic neurons of the hippocampus are severely affected in $\mathrm{AD}$ and degeneration of these neurons might be an early event in the pathogenesis of this disease in humans [56] and animal models of AD [57]. Degeneration of cholinergic neurons in the basal forebrain, which includes the NBM and the associated loss of cholinergic neurotransmission in the cerebral cortex and other areas significantly contribute to the deterioration in cognitive functions [58]. In $\mathrm{AD}$, dysfunctions of the noradrenergic locus coeruleus and the serotonergic dorsal raphe nucleus have also been reported [59]. There seems to 
be a multifocal disease pathology in dementia-related disorders. While some of the memory-circuit related regions are involved in the cognitive aspects of dementia, others are linked to the affective aspects such as the monoaminergic systems [60].

\section{Modulating memory through brain stimulation}

Thus far only a few studies have tried to stimulate structures of the memory circuit (Table 1) and have found beneficial effects when applying DBS correspondingly to the fornix $[8-10,62,63]$, entorhinal cortex $[21,64]$ and NBM $[65,66]$. The fornix and entorhinal cortex are both directly connected to the hippocampus (Fig. 1). The NBM has strong projections not only to the neocortex including primary and secondary visual, auditory, somatosensory, and higher association cortex, but also the hippocampus [67].

\section{Preclinical studies}

The use of animal models has proven its importance in the development and refinement of DBS as a therapy in neuropsychiatry $[68,69]$. The evaluation of different target structures for DBS can be performed in rodents to exclude structures which are less effective. Besides, various stimulation parameters can be tested in animals to define the most optimal stimulation settings producing therapeutic benefits. Moreover, potential side-effects, as well as the underlying mechanisms of DBS can be investigated utilizing animal models.

In line with this, rats treated with corticosterone, a known suppressor of neurogenesis, were stimulated in the anterior nucleus of the thalamus for $1 \mathrm{~h}$ with $2.5 \mathrm{~V}, 130 \mathrm{~Hz}$ and $90 \mu$ s pulse width while being under general anaesthesia [70]. The authors chose this model, in order to investigate, whether high frequency stimulation can restore the disruption of neurogenesis, which may occur as a consequence of pathological conditions. Stimulation was bilateral as well as bipolar. The voltage of $2.5 \mathrm{~V}$, however, is unusually high and it is also more common to use current-based stimulations in animal research. Stimulations with voltage do not guarantee a constant current over time, due to the changes in impedance of the electrodes. In preclinical research it is important to deliver a controlled amount of current, in order to eliminate variations between the subjects. The group size of the stimulated animals was also rather small in this study $(n=4)$. Nevertheless, the authors found that with high frequency stimulation, cell division in the subgranular layer of the hippocampus significantly increased 28 days after the last BrdU injection. Stimulation was therefore able to reverse the suppression of neurogenesis in corticosterone-treated animals when applied sufficiently in advance. Since interventions that increase hippocampal neurogenesis have been associated with enhanced behavioural performance, the authors hypothesized that it may be possible to use electrical stimulation to treat conditions associated with memory impairment. 

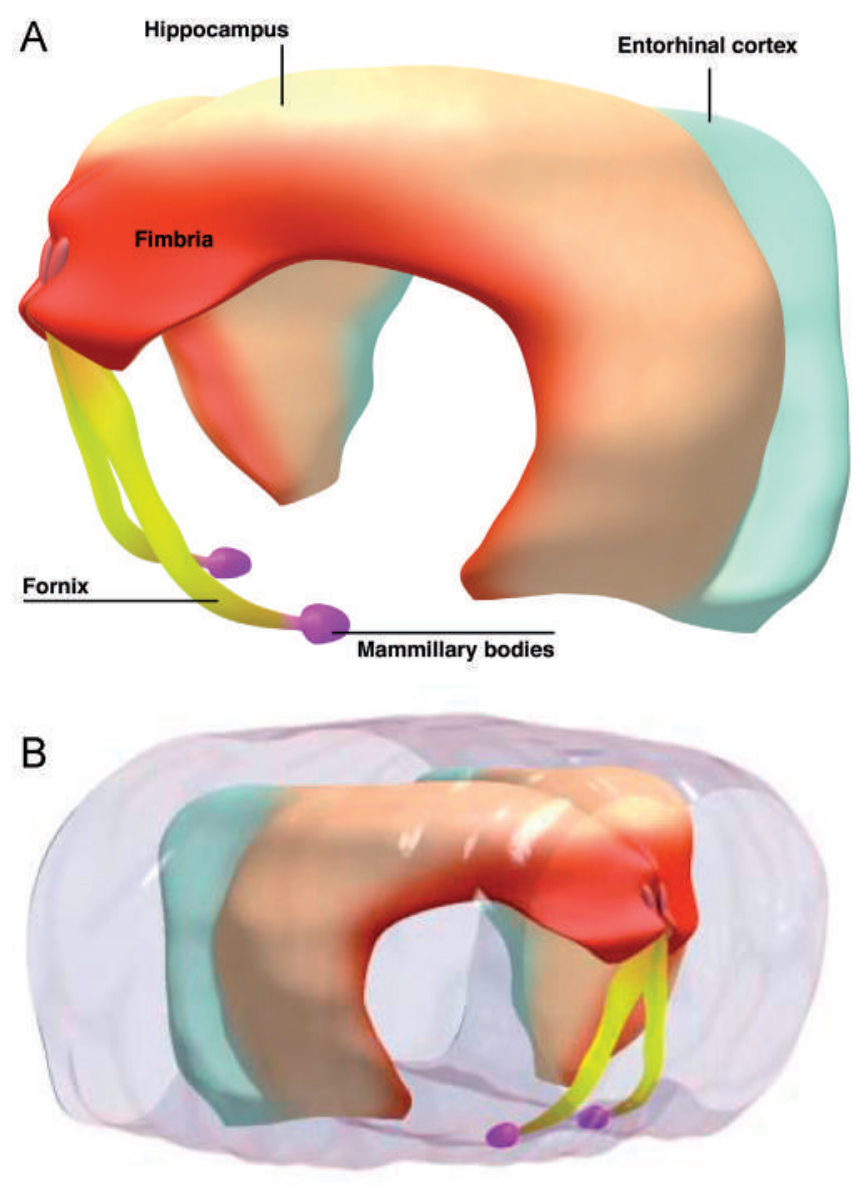

Figure 1: (A) This picture illustrates the anatomy of the mammillary bodies, fornix, fimbria, hippocampus and entorhinal cortex in the rat brain according to the rat brain atlas of Paxinos and Watson (1998). These structures are important components of the Papez circuit and are considered to be involved in the neuronal basis of memory. Especially structures like the hippocampus are involved in spatial learning and memory. Projections within this circuit are mostly cholinergic and glutamatergic. (B) 3D webanimation of (A). The classical idea of the Papez circuit is that the entorhinal cortex projects to the hippocampi, which pass the information on to the fimbria. Efferents are then bundled in the fornices and reach the mamillary bodies. Through the mammillothalamic tract information reaches the anterior nucleus of the thalamus, which is not illustrated here. A functional interaction between hippocampus and anterior thalamic nucleus is required for spatial memory and conditional learning [61]. See (A) for the colour coding of the structures. 


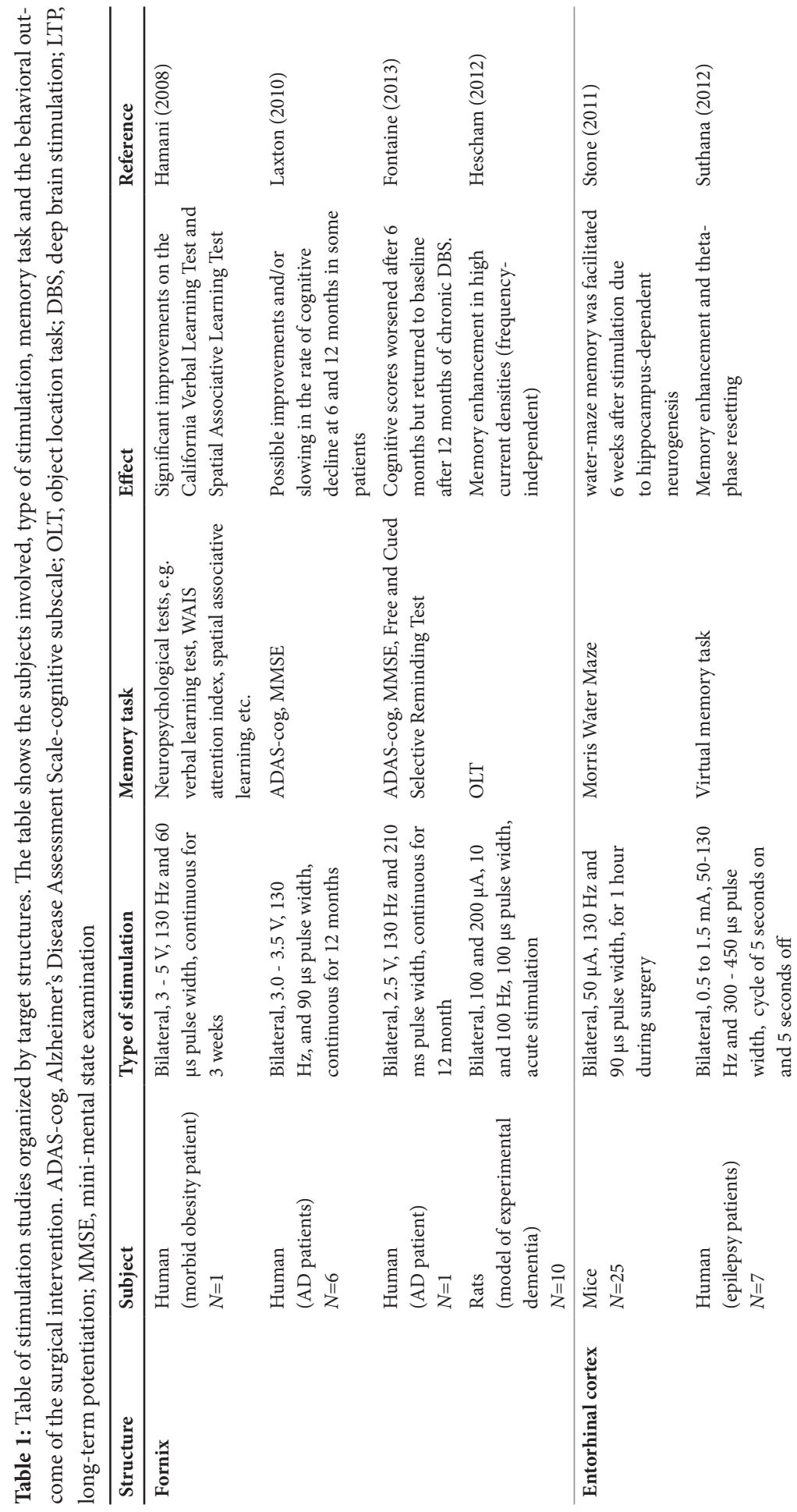




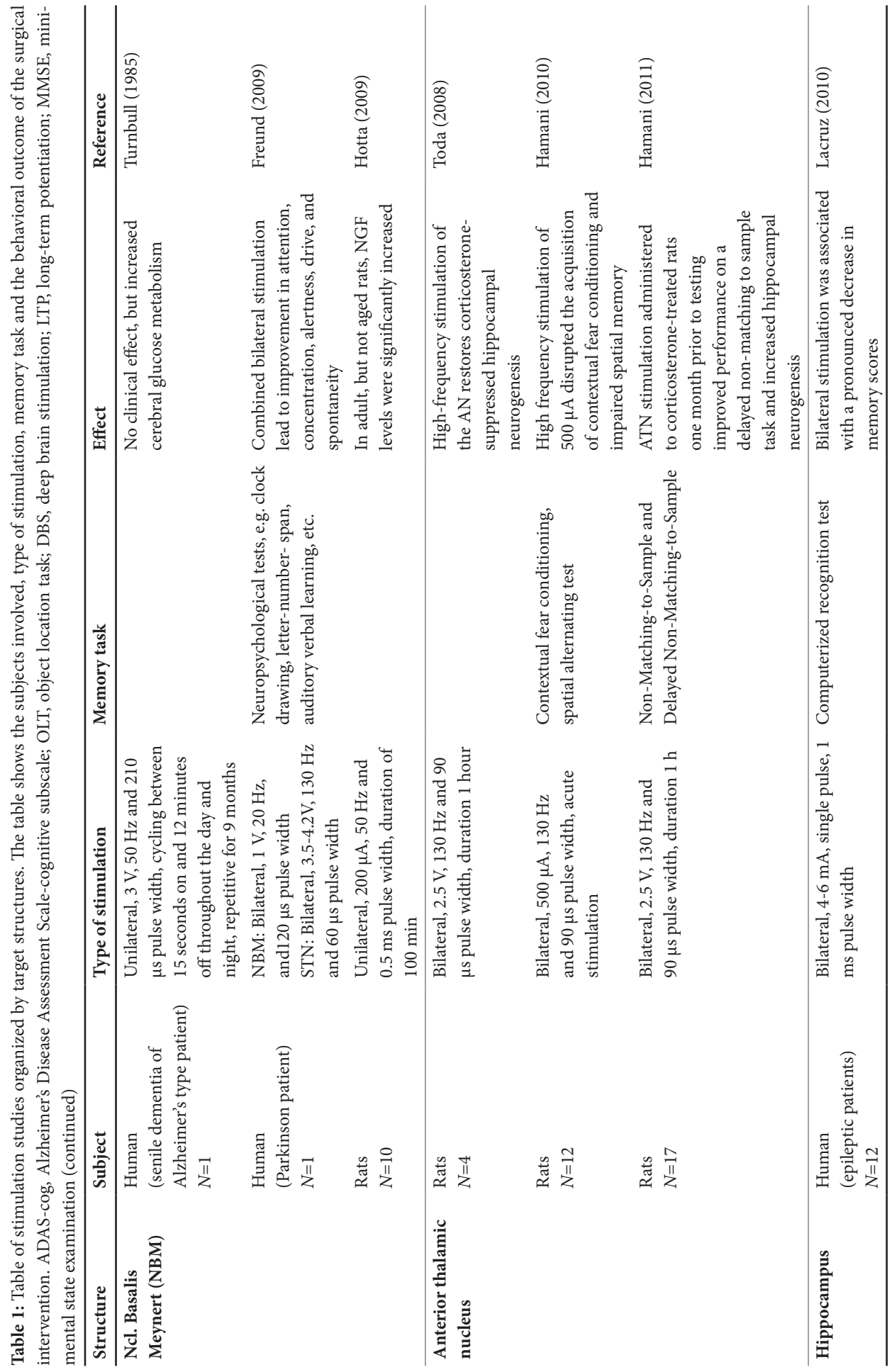


Therefore, in their next study corticosterone-treated rats were again stimulated with the stimulation parameters mentioned above [22]. The authors showed that DBS of the anterior thalamic nuclei applied one month prior to testing improved memory performance on a delayed non-matching to sample task. In contrast, no changes in this task were observed in animals that were tested a few days after surgery, suggesting that stimulation of the anterior thalamic nuclei only induces long-term plastic changes. However, these results should be treated with care, since the aforementioned voltagebased stimulation parameters do not ascertain constant current amplitudes.

In an earlier publication, the same research group showed that acute bilateral, monopolar stimulation of the anterior nucleus of the thalamus, at a relatively high current $(500 \mu \mathrm{A}), 130 \mathrm{~Hz}$ and $90 \mu \mathrm{s}$ pulse width, disrupted the acquisition of contextual fear conditioning and impaired performance on a spatial alternating task (four-arm maze) in rats [71]. Testing started 2 weeks after surgery and even with parameters generating a charge density that approximated the one used in clinical practice $(100 \mu \mathrm{A})$, memory performance of DBS rats was not enhanced. The authors suggest that stimulation with too high current density causes a depolarization block. This finding should be carefully considered in light of the use of anterior thalamus DBS in patients for memory enhancement. It could also be argued that the time between surgery and testing was not sufficient for long-term plastic changes to occur, since stimulation effects on neurogenesis are only observed 4 weeks after surgery $[22,70]$.

Contrary to this, animal studies have also highlighted DBS targets producing clear memory enhancing effects. For example, in a rat model of experimental dementia bilateral DBS of the forniceal region, enhanced spatial memory performance in the object location task [8]. Thus, DBS reversed the memory impairing effects of scopolamine when compared to sham rats in high current densities (both $200 \mu \mathrm{A}$ and $100 \mu \mathrm{A}$ were effective and independent of frequency, $100 \mu$ s pulse width). The bilateral electrodes were all implanted in the vicinity of the fornix. No differences between the ventrally, medially or laterally placed electrodes were found in terms of behavioral outcome and stimulation settings did not produce histological damage or anxiety-related side effects. For the fornix the current hypothesis states that this effect is accomplished by driving the fornix activity through DBS, both orthodromically (away from the soma) as well as antidromically (towards the soma). It was suggested that large myelinated axons usually produce excitatory responses upon electrical stimulation [72].

Another stimulation site inducing memory enhancing effects is the entorhinal cortex. In rodents, the effects of entorhinal cortex DBS have thus far only been reported after chronic stimulation [21]. The entorhinal cortex is strongly connected to the dentate gyrus via the perforant pathway. Either 1.5 or 6.5 weeks after bilateral stimulation of the entorhinal cortex for 1 hour at $50 \mu \mathrm{A}, 130 \mathrm{~Hz}$ and $90 \mu$ s pulse width (while being under general anaesthesia), they evaluated the effects on neurogenesis and memory 
performance. The current density of $50 \mu \mathrm{A}$ is considered as rather low. With the help of the proliferation marker BrdU, the authors found that DBS increased proliferative activity in the dentate gyrus after 6.5 weeks. These newly generated cells survived for several weeks and acquired normal dentate granule cell morphology. Using immunohistochemical approaches, the authors found that, once sufficiently mature, these stimulation-induced neurons integrated into hippocampal circuits, which was associated with an enhanced performance of the stimulated rats in a spatial water maze task. Moreover, because beneficial effects of entorhinal cortex stimulation could be inhibited by blocking neurogenesis with the DNA alkylating agent Temozolomide, a causal relationship between stimulation-induced promotion of adult neurogenesis and enhanced spatial memory can be assumed.

These findings suggest that neurogenesis can be enhanced by stimulation of different regions in the memory circuit (i.e. entorhinal cortex and anterior thalamus). No studies have been conducted in which neurogenesis markers were evaluated after fornix stimulation.

In the context of $\mathrm{AD}$-associated degeneration of the basal forebrain and the dysregulations in nerve growth factor (NGF) levels [73], it seems to be of particular interest to examine the effects of DBS within one of the basal forebrain structure, such as the NBM. It is acknowledged that survival of cholinergic basal forebrain neurons is dependent upon NGF and its high- and low-affinity receptors. Hotta and colleagues [74] therefore applied unilateral electrical stimulation of the NBM $(200 \mu \mathrm{A}, 50 \mathrm{~Hz}$ and $0.5 \mathrm{~ms}$ pulse width for a duration of $100 \mathrm{~min}$ ) in anesthetized rats. NGF levels were measured via a microdialysis probe in the parietal cortex. NGF is known to be essential not only for the survival but also maintenance of neurons and is especially down-regulated in the NBM in AD [73]. The authors found that in adult, but not aged rats, NGF levels were significantly increased ipsilaterally to the stimulation, confirming a potential effect of DBS on neurotrophin release [74]. The question here is whether such a substantial response can also be seen in an animal model of dementia, in which the NGF levels are already affected.

\section{Experimental findings in humans}

The hippocampus is a central structure in the so-called memory circuit and one would assume that hippocampal stimulation would facilitate memory performance. In epileptic patients, the hippocampus was stimulated using $1 \mathrm{~ms}$ single pulse electrical stimulation, intensity ranging between 4-6 mA of bilateral implanted intracerebral depth electrodes [75]. This stimulation paradigm was enforced in order to identify human epileptogenesis and to mimic the physiological stimulation, where cortical networks receive one stimulus before returning to their resting state. Computer-controlled electrical stimulation and recognition memory tests (which included written words, 
geometrical drawings and faces) were performed while patients were connected to multi-channel video-telemetry. One ms single pulses of electrical stimulation were time-locked to item presentation on a computer screen during encoding, recognition or both. However, major deficits were found after bilateral hippocampal stimulation in episodic memory. The findings confirm that localized and brief $1 \mathrm{~ms}$ electrical pulses can disrupt memory function and further support the notion that hippocampal structures are highly relevant to processing episodic memory. The hippocampus, therefore, does not seem to represent a good target structure for DBS in memory-related disorders.

On the contrary, when stimulating the in-/output of the hippocampus, namely the fornix, investigators found an immediate effect on memory [9]. In a case study the authors bilaterally stimulated the fornix/hypothalamic area in a patient suffering from morbid obesity. Stimulation resulted immediately in the generation of detailed autobiographical memories. Also significant improvements on the California Verbal Learning Test and Spatial Associative Learning Test were observed after 3 weeks of continuous stimulation. Electroencephalographic (EEG) source localization elucidated that in this specific case DBS with 3-5 V, $130 \mathrm{~Hz}$ and $60 \mu$ s pulse width evoked neuronal activity in the medial temporal lobe structures like the hippocampus. Following this interesting finding, the authors have performed a phase I trial of fornix DBS in AD patients (see section on clinical studies).

The enthorhinal cortex, which provides the major input to the hippocampus, has also been stimulated in a recent human study with seven pharmacoresistant epilepsy patients. Bilateral intracranial depth electrodes were implanted in the entorhinal cortex region in order to define the area of seizure onset [64]. When subjecting these patients to cognitive testing, Suthana and colleagues found that spatial learning in humans was enhanced in a virtual spatial memory task. Stimulation was acute with biphasic rectangular pulses set below the threshold for afterdischarge. In addition, stimulation parameters were bipolar at a frequency of $50-130 \mathrm{~Hz}$ and a pulse width of 300-450 $\mu$ s with a $5 \mathrm{~s}$ on and a $5 \mathrm{~s}$ off cycle. The current densities ranged from 0.5 to $1.5 \mathrm{~mA}$. Although the cyclic stimulation settings are not often applied in clinical practice of DBS, the authors found that entorhinal stimulation with $50 \mathrm{~Hz}$ led to a theta phase resetting measured through hippocampal depths electrodes. The theta rhythm $(3-8 \mathrm{~Hz})$ is a large EEG potential, which can be recorded from the hippocampus of humans and rodents. During working memory tasks, stimulus presentation induces shifts in the phase of the hippocampal theta-band oscillation and it is hypothesized that resetting theta activity facilitates long-term potentiation by allowing the best possible encoding of relevant incoming stimuli. A potential mechanism for this effect could be neural hijacking. The evidence for the phenomenon of neural hijacking has been extensively described for the microstimulation of the motor cortex in rhesus monkeys (see review 
[76]). The hypothesis states that DBS eliminates natural cortical output activity and replaces it with stimulus-evoked activity.

Taken together, these experimental findings suggested that the fornix and the entorhinal cortex may be considered as interesting targets for dementia, which corresponds to the findings from experimental animal studies. On the other hand, the hippocampus appeared to be a less appealing structure for DBS to enhance memory performance.

\section{Clinical studies}

Laxton et al. [10] performed the first DBS study in patients with AD. Six Patients with mild AD were implanted with electrodes in the area of the fornix/hypothalamus and after an intra-operative evaluation of stimulation, patients were subjected to chronic high frequency DBS for a period of 12 months. Subjects were stimulated with 3.0-3.5 V, $130 \mathrm{~Hz}$ and $90 \mu$ s pulse width. The authors found that the application of DBS in the hypothalamus/fornix triggered neural activity in the entorhinal and hippocampal areas. PET scans revealed an early and striking reversal of the impaired glucose metabolism in the temporal and parietal lobes that was maintained after 12 months of chronic stimulation. After evaluating the AD Assessment Scale cognitive subscale (ADAS-cog) and the Mini Mental State Examination (MMSE), the authors concluded that especially patients with mild cognitive impairments might benefit from this therapy. They showed possible improvements and slowing the progression of memory loss at 6 and 12 months, respectively. When comparing the rate of decline in the 11 months preceding surgery to the 11 months after surgery a decrease in the rate of decline from a mean rate of 2.8 to 0.8 points in the MMSE across the 6 patients can be observed. After 1 year of continuous DBS, the functional connectivity analysis demonstrated increased cerebral metabolism in cortical-subcortical and cortical-hippocampal networks. This increase after 1 year of DBS was correlated with less decline in global cognition and memory as measured with the ADAS-cog [62]. The authors reported adverse effects only in their first publication [10], but did not mention them in their follow-up study [62]. Side effects were autonomic and cardiovascular in nature and occurred merely in high stimulation settings above $7 \mathrm{~V}$. Chronic stimulation was therefore chosen as $50 \%$ of the voltage threshold for adverse effects. No patient developed sleep disturbances, weight changes or evidence of hypothalamic dysfunction [10]. Since only 6 patients were tested in this first trial, new clinical trials are currently implemented in the ADvance study, which is sponsored by the Functional Neuromodulation Ltd company and is being conducted at sites across the U.S. and in Canada. The study will involve 20 mild AD patients, with DBS electrodes implanted in the fornix. The stimulation device will be turned on immediately for half of the patients and for the other half only after 12 months. It is a double-blind randomized controlled feasibility study to evaluate acute and long-term safety. Efficacy outcomes will be measured at 12 months 
and identify improvements in ADAS-cog, clinical dementia rating and changes in the glucose metabolism assessed by FDG-PET.

A prospective pilot study in France has already investigated the feasibility of applying DBS of the fornix in AD patients [63]. Since inclusion criteria are strict, e.g. patients must be under 70 years of age, have a MMSE between 20 and 24 (mild cognitively impaired), show a predominant impairment of episodic memory and fulfil the AD DSM IV criteria for less than 2 years, only 9 out of 110 recruited patients were found suitable to enter the study. In the end, only one patient agreed to undergo surgery. This number is very low, considering the fact that alternative efficient treatments are currently lacking.

Attention has also been drawn to the NBM as a potential target structure for DBS in $\mathrm{AD}$, since it has wide cholinergic projections to the neocortex and the hippocampus. In the pathogenesis of AD the NBM degenerates, leading to decreased cholinergic transmission and ultimately to cognitive decline in patients [77]. In this regard, Turnbull and colleagues [66] used chronic, cyclical, unilateral, monopolar stimulation of the left NBM in a patient with mild to moderate $\mathrm{AD}$. It has been shown that low-frequency stimulation has excitatory actions [78] and neuromodulation might therefore be a strategy for enhancing residual NBM cholinergic output. Stimulation parameters were set to $3 \mathrm{~V}, 50$ $\mathrm{Hz}$, and $210 \mu \mathrm{s}$, cycling between 15 seconds on and 12 minutes off throughout the day and night. The authors found that the patient had no clinical response to the stimulation 8 months after the procedure. However, the NBM was targeted indirectly using atlas coordinates and electrode placement was only verified by a computed tomography (CT) scan, in which the NBM was not visible. Another issue of debate is the choice for specific stimulation parameters: unilateral, short-lasting and intermittent stimulation, which was delivered for a total of 30 minutes every 24 hours. No rationale for these stimulation parameters was provided by the authors. By today's standard these settings are highly unusual, given that nowadays stimulation is often chronically applied to patients. Nevertheless, stimulation had an effect on cerebral glucose metabolism. Using the patient's unstimulated contralateral hemisphere as a control, Turnbull and colleagues [66] were able to compare his pre- and postoperative FDG-PET scans. The preoperative scan was obtained 4 months before surgery and the postoperative scan was obtained 2 months after the initiation of stimulation, respectively. The FDG-PET scans of the right hemisphere showed that glucose metabolism in the frontal, temporal, parietal, and occipital lobes decreased by $21 \%, 24 \%, 10 \%$, and $7.5 \%$, respectively. In contrast, glucose use in the stimulated left hemisphere had decreased by only $12 \%$ in the frontal lobe and $4.1 \%$ in the occipital lobe. No change was found in the parietal lobe and glucose use was increased by $1.5 \%$ in the temporal lobe.

Freund and colleagues [65] have also stimulated the NBM (in addition to the subthalamic nucleus) in a patient suffering from Parkinson's disease dementia. Imaging and 
post-mortem studies have identified that degeneration of the NBM appears early in the pathogenesis and worsens progressively [79]. With bilateral stimulation settings of 1 $\mathrm{V}, 20 \mathrm{~Hz}$ and $120 \mu$ s the patient showed sustained improvement in various aspects of cognitive functioning, such as concentration, attention, alertness, apraxia, ataxia and memory. No adverse effects were reported in this study. The authors also hypothesized that DBS of this structure induces neuroprotective effects through the release of NGF into the NBM [80]. However, NGF was not measured in this study. A clinical study is currently being performed with six mild AD patients at the Uniklinik Köln in Germany (NCT01094145). In this study, two weeks after DBS electrodes in the NBM, the conventional stimulation will take place as a doubleblind, randomized change between on and off stimulation periods. Throughout the one-year observation-period, the patients will be followed closely to monitor the effects of DBS on their cognitive abilities, psychopathological well being, the quality of life, praxia and nutritional condition using standardized neurological and psychiatric rating scales.

\section{DISCUSSION}

DBS as a potential therapy for memory-related disorders is currently being explored in clinical trials. Especially DBS of the fornix and the NBM are investigated as potential treatments for dementia. Based on the limited data that are available it is assumed that DBS of the fornix might provide symptomatic relief for verbal recollection, recall and recognition as well as episodic memory, while NBM stimulation might modulate apraxia and alertness. Promising results have also been observed in enthorinal cortex stimulation, even though no clinical trials are examining the effects of entorhinal cortex DBS as memory enhancer at present.

An important issue that needs to be raised is that up to now, most DBS studies in psychiatric disorders were first conducted in humans. Since the clinical data and the findings from animal studies show similarities, animal models can be of important value to find potential new DBS targets and settings for memory enhancement (see review [68]). From present DBS studies it is evident that structures within the brain show different responses to stimulation parameters. Moreover, clinical case studies and animal studies have shown that DBS of different target structures may enhance memory functions by different mechanisms of action (Fig. 2).

The fornix, for example, is not dependent on frequency but rather on current densities. In preclinical research relatively high current densities of 100 or $200 \mu \mathrm{A}$ produced beneficial effects on memory [81]. In clinical trials $\mathrm{AD}$ patients have been stimulated with 2.5-3.5 V [10,63], which is generally accepted as moderate-sized voltage in the treatment of psychiatric disorders with DBS. The investigators of the clinical studies 


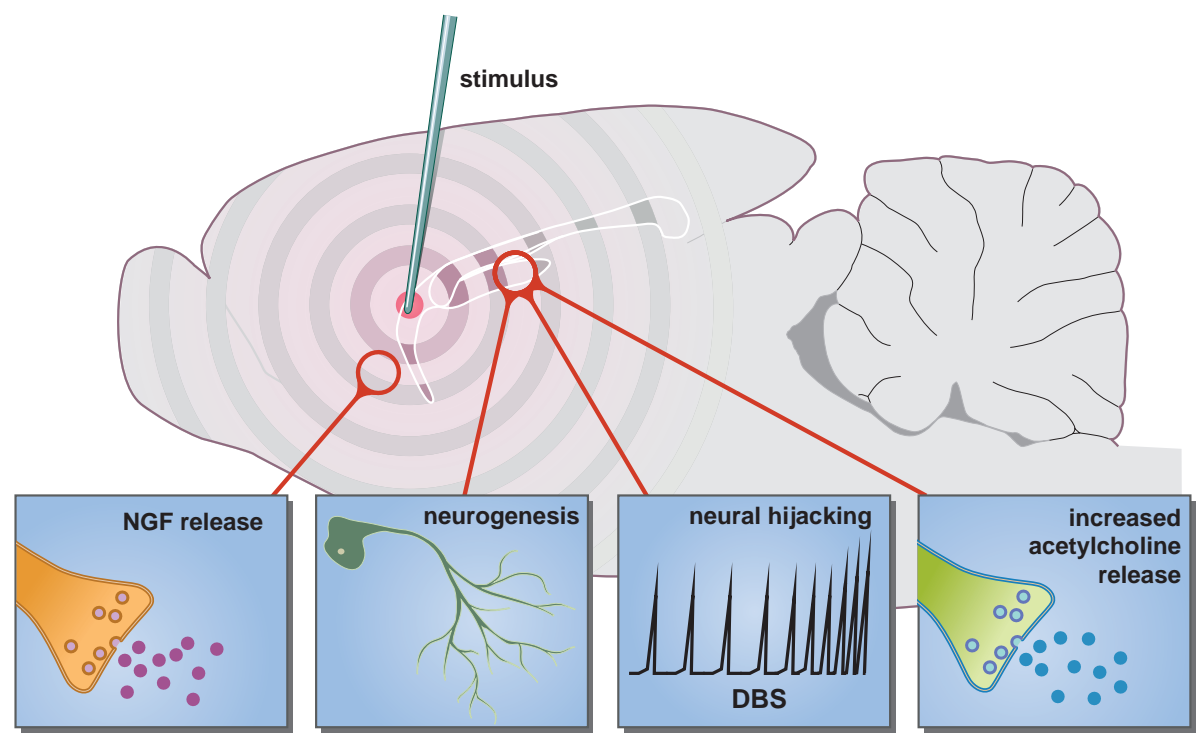

Figure 2: Schematic representation of the potential mechanisms involved in enhancing memory functions by deep brain stimulation. Stimulation of a target area within the memory circuit (e.g. fornix) can provoke NGF release in the NBM, hippocampal-dependent neurogenesis, neural hijacking by resetting theta activity and increased acetylcholine release within the hippocampal region.

chose these parameters in order to abolish adverse effects. With these settings the authors described possible improvements and/or slowing in the rate of cognitive decline at 6 and 12 months in some patients [10,63]. The reason why the fornix is not dependent on frequency can be related to its morphology. The fornix consists of a bundle of myelinated fibres, known as white matter. This white matter is activated upon stimulation, regardless whether stimulation is applied with high or low frequency, and produces an excitatory response. As seen in the rodent study, relatively high current is needed to produce beneficial effects on memory, most probably because the fornix is a small target area and electrodes were only located in its vicinity [8]. In the same study, memory deficits induced by the muscarinic acetylcholine receptor antagonist scopolamine were reversed after fornix stimulation. Thus, DBS of the fornix might increase acetylcholine release in the hippocampus.

The NBM, on the other hand, is rather dependent on frequency. To the best of our knowledge, no animal study has been conducted, in which memory was assessed after NBM stimulation. It has been shown, however, that low frequency stimulation of 50 $\mathrm{Hz}$ induces increased NGF release from the NBM in anaesthetized rats, a neurotrophin responsible for survival and maintenance of neurons [74]. Since typical neuronal discharge rates of the NBM are of low frequency and it is known that cell bodies are usually activated by low frequency, stimulations with $50 \mathrm{~Hz}$ [66] and $20 \mathrm{~Hz}$ [65] were 
applied in demented patients. In fact, in a demented Parkinson's patient the combined bilateral stimulation of subthalamic nucleus and NBM led to improvement in attention, concentration, alertness, drive and spontaneity [65]. Nevertheless, this has only been shown in a single case study and more evidence is needed. Similar to the observations of the rodent study, the authors hypothesized that an increase of neurotrophin release from the NBM upon stimulation correlates with improved memory performance [65, 80].

Next, stimulation of the entorhinal cortex demonstrates effects on neurogenesis, as well as theta-phase resetting within the hippocampus when stimulated with low current densities $[21,64]$. Rodents were stimulated in the entorhinal cortex with $50 \mu \mathrm{A}$ [21] and humans with 0.5-1.5 mA [64], both considered as relatively low current densities. With these stimulation parameters increased neurogenesis in the rodent study was associated with enhanced spatial memory in the water maze [21], while in the human study hippocampal theta-phase resetting was directly related to enhanced memory performance in a virtual memory task [64]. Besides, both studies utilized high frequency stimulation. For the entorhinal cortex it can be hypothesized that DBS with low current and high frequency has an effect on neighbouring axons. Extensive evidence supports the speculation that axons have a lower threshold for activation by electrical stimulation than cell bodies and usually respond to high frequency stimulation. The fibres from the perforant pathway might be activated upon stimulation inducing neurogenesis in the dentate gyrus and neural hijacking might take place. Since alterations in neurogenesis have been extensively described in animal models of AD and its decline is to some extent responsible for learning and memory impairments (see review [82]), the entorhinal cortex might be an interesting structure for future DBS studies.

Finally, the anterior nucleus of the thalamus seems to be a target producing contradictory stimulation effects on memory. On the one hand, effects on neurogenesis have been observed following high frequency stimulation of $130 \mathrm{~Hz}[22,70]$, but on the other side application of high current densities $(500 \mu \mathrm{A})$ within this structure produced memory disruption [71]. Interestingly this nucleus is being targeted for treating severe epilepsy, based on the promising data from the SANTE (Stimulation of the Anterior Nucleus of the Thalamus for Epilepsy) trial. In this study the safety and effectiveness of bilateral stimulation of the anterior nucleus of the thalamus is examined as adjunctive therapy for reducing the frequency of seizures in adults diagnosed with epilepsy (NCT00101933). The 110 participants of this study were randomized to relatively high stimulation at $5 \mathrm{~V}$ or no stimulation at $0 \mathrm{~V}$ (control), using $90 \mu$ s pulses, 145 pulses/s, 1 min on, and 5 min off. Indeed, this study demonstrated a beneficial and sustained effect of DBS on seizure frequency. Neuropsychological testing showed no group differences on cognitive functions, however significantly more participants of the stimulated group reported depression and memory problems as adverse events [83]. These behavioral 
effects are partially in line with findings from animal studies, in which stimulation with a relatively high current density of $500 \mu \mathrm{A}$ also disrupted memory performance.

Similarly, experimental findings in epileptic patients indicated that hippocampal single pulse stimulation might impair rather than facilitate memory functions [84, 85]. Stimulations within the hippocampus might cause an acute depolarization block, which possibly disrupts formation and recall of recent episodic memory.

From this we can conclude that consideration of ethics in clinical DBS research is of growing importance. The cost/benefit ratio should be carefully evaluated for each patient. For the treatment of dementia-related disorders, data suggests that DBS of the fornix is most beneficial for patients who are less severely affected [10]. DBS might therefore be more suitable for patients in the early stages of the pathogenesis of memory impairment, and not after drug treatment fails. This might be contrary to DBS treatment in movement disorders, where DBS applied to treatment-resistant patients has already been shown to be an effective therapy in alleviating motor symptoms. Since at the moment not much data is available showing clear beneficial effects of DBS in $\mathrm{AD}$, patients might not be motivated to undergo this therapy. Up to now, DBS studies in memory-related disorders were mainly safety studies, with small sample sizes and no control group. Furthermore, suitable cognitive re-evaluations were lacking in some studies $[65,66]$. Since no clear conclusion can be drawn from these findings, we expect that patients favour drug treatment above undergoing brain surgery. The difficulty in finding participants, who fulfil the strict inclusion criteria for this type of studies has been discussed earlier [63].

Since DBS in Parkinson's disease patients has been applied for more than a decade now, a lot of evidence has already been reported about adverse effects. For example, it has been shown that approximately $0.5 \%$ of patients implanted with DBS electrodes within the subthalamic nucleus commit suicide due to the development of a major depression [86]. For AD or other demented patients, very few adverse effects have been described so far. Since the target regions of DBS to enhance memory functions are different from the ones used in movement disorders, also no speculations can be made. Nevertheless, one major advantage of DBS is its reversible nature, since stimulation settings can be modulated in a way to keep adverse effects at a minimal level.

Besides showing therapeutic effects, another question is whether DBS can be disease-modifying. In the context of extensive experience of DBS in Parkinson's patients, evidence that DBS influences the underlying neurodegenerative process is up to now very weak. In animal research, however, studies in both rats and monkeys have demonstrated that DBS of the subthalamic nucleus can prevent the degeneration of nigral dopamine neurons from the insult produced by dopamine depleting neurotoxins [8790]. Nevertheless, animal models of neurodegeneration following the administration of 
toxins do not adequately reflect the neurodegenerative processes in humans and these results should not be over-interpreted.

Up to now, contributions of lesion studies within the memory circuit have provided solid groundwork in understanding the neuroanatomy of memory [37-39, 42, 52, 91, 92]. The next challenge is to use these findings and derive target structures for the application of DBS in diseases characterized by dementia. The good predictive and construct validity of some animal models for understanding the effects and neural substrates underlying DBS suggest that preclinical animal models are an important tool for accelerating and validating clinical application of DBS. Nevertheless, it is still premature to conclude that DBS can be used in the treatment of AD, and the field will wait for the results of ongoing clinical trials.

\section{ACKNOWLEDGEMENTS}

The authors wish to thank Geertjan van Zonneveld for the 3D reconstruction of the hippocampus as well as the according animation. This work was supported by the Internationale Stichting Alzheimer Onderzoek (ISAO). 


\section{REFERENCES}

1. Lobo A, Launer LJ, Fratiglioni L, Andersen K, Di Carlo A, Breteler MM, et al. Prevalence of dementia and major subtypes in Europe: A collaborative study of population-based cohorts. Neurologic Diseases in the Elderly Research Group. Neurology. 2000;54(11 Suppl 5):S4-9. Epub 2000/06/15. eng.

2. Plassman BL, Langa KM, Fisher GG, Heeringa SG, Weir DR, Ofstedal MB, et al. Prevalence of dementia in the United States: the aging, demographics, and memory study. Neuroepidemiology. 2007;29(1-2):125-32. Epub 2007/11/03. eng.

3. Buckner RL, Snyder AZ, Shannon BJ, LaRossa G, Sachs R, Fotenos AF, et al. Molecular, Structural, and Functional Characterization of Alzheimer's Disease: Evidence for a Relationship between Default Activity, Amyloid, and Memory. The Journal of Neuroscience. 2005;25(34):7709-17.

4. Thies W, Bleiler L. 2011 Alzheimer's disease facts and figures. Alzheimer's \& dementia : the journal of the Alzheimer's Association. 2011;7(2):208-44.

5. Dubois B, Feldman HH, Jacova C, Cummings JL, DeKosky ST, Barberger-Gateau P, et al. Revising the definition of Alzheimer's disease: a new lexicon. The Lancet Neurology. 2010;9(11):1118-27.

6. Brookmeyer R, Gray S, Kawas C. Projections of Alzheimer's disease in the United States and the public health impact of delaying disease onset. American journal of public health. 1998;88(9):133742. Epub 1998/09/16. eng.

7. Qaseem A, Snow V, Cross JT, Forciea MA, Hopkins R, Shekelle P, et al. Current Pharmacologic Treatment of Dementia: A Clinical Practice Guideline from the American College of Physicians and the American Academy of Family Physicians. Annals of Internal Medicine. 2008;148(5):370-8.

8. Hescham S, Lim LW, Jahanshahi A, Steinbusch HW, Prickaerts J, Blokland A, et al. Deep brain stimulation of the forniceal area enhances memory functions in experimental dementia: The role of stimulation parameters. Brain Stimul 2013;6(1):72-7.

9. Hamani C, McAndrews MP, Cohn M, Oh M, Zumsteg D, Shapiro CM, et al. Memory enhancement induced by hypothalamic/fornix deep brain stimulation. Annals of Neurology. 2008;63(1):119-23.

10. Laxton AW, Tang-Wai DF, McAndrews MP, Zumsteg D, Wennberg R, Keren R, et al. A phase I trial of deep brain stimulation of memory circuits in Alzheimer's disease. Annals of Neurology. 2010;68(4):521-34.

11. Boggio PS, Valasek CA, Campanhã C, Giglio ACA, Baptista NI, Lapenta OM, et al. Non-invasive brain stimulation to assess and modulate neuroplasticity in Alzheimer's disease. Neuropsychological Rehabilitation. 2011;21(5):703-16.

12. Wichmann T, DeLong MR. Deep Brain Stimulation for Neurologic and Neuropsychiatric Disorders. Neuron. 2006;52(1):197-204.

13. Temel Y, Visser-Vandewalle V. Targets for deep brain stimulation in Parkinson's disease. Expert Opinion on Therapeutic Targets. 2006;10(3):355-62.

14. Andrade DM, Zumsteg D, Hamani C, Hodaie M, Sarkissian S, Lozano AM, et al. Long-term followup of patients with thalamic deep brain stimulation for epilepsy. Neurology. 2006;66(10):1571-3.

15. Houeto JL, Karachi C, Mallet L, Pillon B, Yelnik J, Mesnage V, et al. Tourette's syndrome and deep brain stimulation. Journal of Neurology, Neurosurgery \& Psychiatry. 2005;76(7):992-5.

16. Savica R, Stead M, Mack KJ, Lee KH, Klassen BT. Deep Brain Stimulation in Tourette Syndrome: A Description of 3 Patients With Excellent Outcome. Mayo Clinic Proceedings. 2012;87(1):59-62.

17. Bewernick BH, Hurlemann R, Matusch A, Kayser S, Grubert C, Hadrysiewicz B, et al. Nucleus Accumbens Deep Brain Stimulation Decreases Ratings of Depression and Anxiety in TreatmentResistant Depression. Biological Psychiatry. 2010;67(2):110-6. 
18. Lozano AM, Mayberg HS, Giacobbe P, Hamani C, Craddock RC, Kennedy SH. Subcallosal Cingulate Gyrus Deep Brain Stimulation for Treatment-Resistant Depression. Biological Psychiatry. 2008;64(6):461-7.

19. Malone Jr DA, Dougherty DD, Rezai AR, Carpenter LL, Friehs GM, Eskandar EN, et al. Deep Brain Stimulation of the Ventral Capsule/Ventral Striatum for Treatment-Resistant Depression. Biological Psychiatry. 2009;65(4):267-75.

20. Denys D, Mantione M, Figee M, van den Munckhof P, Koerselman F, Westenberg H, et al. Deep Brain Stimulation of the Nucleus Accumbens for Treatment-Refractory Obsessive-Compulsive Disorder. Arch Gen Psychiatry. 2010;67(10):1061-8.

21. Stone SSD, Teixeira CM, DeVito LM, Zaslavsky K, Josselyn SA, Lozano AM, et al. Stimulation of Entorhinal Cortex Promotes Adult Neurogenesis and Facilitates Spatial Memory. The Journal of Neuroscience. 2011;31(38):13469-84.

22. Hamani C, Stone SS, Garten A, Lozano AM, Winocur G. Memory rescue and enhanced neurogenesis following electrical stimulation of the anterior thalamus in rats treated with corticosterone. Exp Neurol. 2011;232(1):100-4. Epub 2011/09/13. eng.

23. Boggio PS, Ferrucci R, Rigonatti SP, Covre P, Nitsche M, Pascual-Leone A, et al. Effects of transcranial direct current stimulation on working memory in patients with Parkinson's disease. J Neurol Sci. 2006;249(1):31-8.

24. Fregni F, Boggio P, Nitsche M, Bermpohl F, Antal A, Feredoes E, et al. Anodal transcranial direct current stimulation of prefrontal cortex enhances working memory. Experimental Brain Research. 2005;166(1):23-30.

25. Köhler S, Paus T, Buckner RL, Milner B. Effects of Left Inferior Prefrontal Stimulation on Episodic Memory Formation: A Two-Stage fMRI-rTMS Study. Journal of Cognitive Neuroscience. 2004;16(2):178-88.

26. Luber B, Kinnunen LH, Rakitin BC, Ellsasser R, Stern Y, Lisanby SH. Facilitation of performance in a working memory task with rTMS stimulation of the precuneus: Frequency- and time-dependent effects. Brain Res. 2007;1128(0):120-9.

27. Boggio PS, Khoury LP, Martins DCS, Martins OEMS, de Macedo EC, Fregni F. Temporal cortex direct current stimulation enhances performance on a visual recognition memory task in Alzheimer disease. Journal of Neurology, Neurosurgery \& Psychiatry. 2009;80(4):444-7.

28. Bentwich J, Dobronevsky E, Aichenbaum S, Shorer R, Peretz R, Khaigrekht M, et al. Beneficial effect of repetitive transcranial magnetic stimulation combined with cognitive training for the treatment of Alzheimer's disease: a proof of concept study. Journal of Neural Transmission. 2011;118(3):463-71.

29. Cotelli M, Calabria M, Manenti R, Rosini S, Zanetti O, Cappa SF, et al. Improved language performance in Alzheimer disease following brain stimulation. Journal of Neurology, Neurosurgery \& Psychiatry. 2011;82(7):794-7.

30. Witter MP, Naber PA, van Haeften T, Machielsen WC, Rombouts SA, Barkhof F, et al. Cortico-hippocampal communication by way of parallel parahippocampal-subicular pathways. Hippocampus. 2000;10(4):398-410. Epub 2000/09/14. eng.

31. Megías M, Emri Z, Freund TF, Gulyás AI. Total number and distribution of inhibitory and excitatory synapses on hippocampal CA1 pyramidal cells. Neuroscience. 2001;102(3):527-40.

32. Aggleton JP DR, Mishkin M. The origin, course, and termination of the hippocampothalamic projections in the macaque. J Comp Neurol. 1986;243(3):409-21.

33. Neave N, Lloyd S, Sahgal A, Aggleton JP. Lack of effect of lesions in the anterior cingulate cortex and retrosplenial cortex on certain tests of spatial memory in the rat. Behavioural Brain Research. 1994;65(1):89-101. 
34. Gemmell C, O'Mara SM. Plasticity in the projection from the anterior thalamic nuclei to the anterior cingulate cortex in the rat in vivo: paired-pulse facilitation, long-term potentiation and short-term depression. Neuroscience. 2002;109(3):401-6. Epub 2002/02/02. eng.

35. Hedberg TG, Stanton PK. Long-term potentiation and depression of synaptic transmission in rat posterior cingulate cortex. Brain Res. 1995;670(2):181-96. Epub 1995/01/30. eng.

36. Aggleton J, Brown M. Episodic memory, amnesia, and the hippocampal-anterior thalamic axis. Behav Brain Sci. 1999;22(3):425-44.

37. Aggleton JP, O’Mara SM, Vann SD, Wright NF, Tsanov M, Erichsen JT. Hippocampal-anterior thalamic pathways for memory: uncovering a network of direct and indirect actions. Eur J Neurosci. 2010;31(12):2292-307. Epub 2010/06/17. eng.

38. Aggleton J, Hunt P, Nagle S, Neave N. The effects of selective lesions within the anterior thalamic nuclei on spatial memory in the rat. Behav Brain Res. 1996;81(1-2):189-98.

39. Moser MB, Moser EI, Forrest E, Andersen P, Morris RG. Spatial learning with a minislab in the dorsal hippocampus. Proc Natl Acad Sci U S A. 1995;92(21):9697-701. Epub 1995/10/10. eng.

40. Célérier A, Ognard R, Decorte L, Beracochea D. Deficits of spatial and non-spatial memory and of auditory fear conditioning following anterior thalamic lesions in mice: comparison with chronic alcohol consumption. European Journal of Neuroscience. 2000;12(7):2575-84.

41. Gaskin S, Tremblay A, Mumby DG. Retrograde and anterograde object recognition in rats with hippocampal lesions. Hippocampus. 2003;13(8):962-9.

42. Broadbent NJ, Squire LR, Clark RE. Spatial memory, recognition memory, and the hippocampus. Proc Natl Acad Sci U S A. 2004;101(40):14515-20. Epub 2004/09/29. eng.

43. Clark RE, Zola SM, Squire LR. Impaired recognition memory in rats after damage to the hippocampus. J Neurosci. 2000;20(23):8853-60. Epub 2000/01/11. eng.

44. Ennaceur A, Neave N, Aggleton JP. Spontaneous object recognition and object location memory in rats: the effects of lesions in the cingulate cortices, the medial prefrontal cortex, the cingulum bundle and the fornix. Experimental Brain Research. 1997;113(3):509-19.

45. Santín L, Rubio S, Begega A, Arias J. Effects of mammillary body lesions on spatial reference and working memory tasks. Behav Brain Res. 1998;102(1-2):137-50.

46. Vann SD, Honey RC, Aggleton JP. Lesions of the mammillothalamic tract impair the acquisition of spatial but not nonspatial contextual conditional discriminations. European Journal of Neuroscience. 2003;18(8):2413-6.

47. Warburton EC, Baird AL, Morgan A, Muir JL, Aggleton JP. Disconnecting hippocampal projections to the anterior thalamus produces deficits on tests of spatial memory in rats. European Journal of Neuroscience. 2000;12(5):1714-26.

48. Whishaw I, Cassel J, Jarrad L. Rats with fimbria-fornix lesions display a place response in a swimming pool: a dissociation between getting there and knowing where. The Journal of Neuroscience. 1995;15(8):5779-88.

49. Tsivilis D, Vann SD, Denby C, Roberts N, Mayes AR, Montaldi D, et al. A disproportionate role for the fornix and mammillary bodies in recall versus recognition memory. Nat Neurosci. 2008;11(7):83442. Epub 2008/06/17. eng.

50. Eijkenboom M, Blokland A, van der Staay FJ. Modelling cognitive dysfunctions with bilateral injections of ibotenic acid into the rat entorhinal cortex. Neuroscience. 2000;101(1):27-39.

51. Oswald C, Good M. The effects of combined lesions of the subicular complex and the entorhinal cortex on two forms of spatial navigation in the water maze. Behav Neurosci. 2000;114(1):211-7.

52. Parron C, Poucet B, Save E. Entorhinal cortex lesions impair the use of distal but not proximal landmarks during place navigation in the rat. Behav Brain Res. 2004;154(2):345-52. 
53. Mair R, Burk J, Porter M. Impairment of radial maze delayed nonmatching after lesions of anterior thalamus and parahippocampal cortex. Behav Neurosci. 2003;117(3):596-605.

54. Parron C, Save E. Comparison of the effects of entorhinal and retrosplenial cortical lesions on habituation, reaction to spatial and non-spatial changes during object exploration in the rat. Neurobiology of Learning and Memory. 2004;82(1):1-11.

55. Rothblat L, Vnek N, Gleason T, Kromer L. Role of the parahippocampal region in spatial and nonspatial memory: effects of parahippocampal lesions on rewarded alternation and concurrent object discrimination learning in the rat. Behav Brain Res. 1992;55(1):93-100.

56. Revett TJ, Baker GB, Jhamandas J, Kar S. Glutamate system, amyloid ss peptides and tau protein: functional interrelationships and relevance to Alzheimer disease pathology. J Psychiatry Neurosci. 2012;37(5):110190. Epub 2012/08/17. Eng.

57. Tiwari V, Patel AB. Impaired glutamatergic and GABAergic function at early age in AbetaPPswePS1dE9 mice: implications for Alzheimer's disease. J Alzheimers Dis. 2012;28(4):765-9. Epub 2011/11/25. eng.

58. Francis PT, Palmer AM, Snape M, Wilcock GK. The cholinergic hypothesis of Alzheimer's disease: a review of progress. J Neurol Neurosurg Psychiatry. 1999;66(2):137-47. Epub 1999/03/10. eng.

59. Trillo L, Das D, Hsieh W, Medina B, Moghadam S, Lin B, et al. Ascending monoaminergic systems alterations in Alzheimer's disease. Translating basic science into clinical care. Neurosci Biobehav Rev. 2013;37(8):1363-79. Epub 2013/05/28. Eng.

60. Esiri MM. The basis for behavioural disturbances in dementia. J Neurol Neurosurg Psychiatry. 1996;61(2):127-30. Epub 1996/08/01. eng.

61. Dumont JR, Petrides M, Sziklas V. Fornix and retrosplenial contribution to a hippocampo-thalamic circuit underlying conditional learning. Behav Brain Res. 2010;209(1):13-20.

62. Smith GS, Laxton AW, Tang-Wai DF, McAndrews MP, Diaconescu AO, Workman CI, et al. Increased Cerebral Metabolism After 1 Year of Deep Brain Stimulation in Alzheimer DiseaseIncreased Cerebral Metabolism After 1 Year of DBS. Arch Neurol. 2012:1-8.

63. Fontaine D, Deudon A, Lemaire JJ, Razzouk M, Viau P, Darcourt J, et al. Symptomatic treatment of memory decline in Alzheimer's disease by deep brain stimulation: a feasibility study. J Alzheimers Dis. 2013;34(1):315-23. Epub 2012/11/22. eng.

64. Suthana N, Haneef Z, Stern J, Mukamel R, Behnke E, Knowlton B, et al. Memory Enhancement and Deep-Brain Stimulation of the Entorhinal Area. New England Journal of Medicine. 2012;366(6):50210.

65. Freund HJ, Kuhn J, Lenartz D, Mai JK, Schnell T, Klosterkoetter J, et al. Cognitive functions in a patient with Parkinson-dementia syndrome undergoing deep brain stimulation. Arch Neurol. 2009;66(6):781-5. Epub 2009/06/10. eng.

66. Turnbull IM, McGeer PL, Beattie L, Calne D, Pate B. Stimulation of the basal nucleus of Meynert in senile dementia of Alzheimer's type. A preliminary report. Appl Neurophysiol. 1985;48(1-6):216-21. Epub 1985/01/01. eng.

67. Bigl V, Woolf NJ, Butcher LL. Cholinergic projections from the basal forebrain to frontal, parietal, temporal, occipital, and cingulate cortices: a combined fluorescent tracer and acetylcholinesterase analysis. Brain Res Bull. 1982;8(6):727-49. Epub 1982/06/01. eng.

68. Hamani C, Temel Y. Deep Brain Stimulation for Psychiatric Disease: Contributions and Validity of Animal Models. Sci Transl Med. 2012;4(142):142rv8.

69. Tan S, Vlamings R, Lim L, Sesia T, Janssen ML, Steinbusch HW, et al. Experimental deep brain stimulation in animal models. Neurosurgery. 2010;67(4):1073-9. Epub 2010/10/01. eng. 
70. Toda H, Hamani C, Fawcett AP, Hutchison WD, Lozano AM. The regulation of adult rodent hippocampal neurogenesis by deep brain stimulation. J Neurosurg. 2008;108(1):132-8.

71. Hamani C, Dubiela FP, Soares JCK, Shin D, Bittencourt S, Covolan L, et al. Anterior thalamus deep brain stimulation at high current impairs memory in rats. Exp Neurol. 2010;225(1):154-62.

72. Ranck JB. Which elements are excited in electrical stimulation of mammalian central nervous system: A review. Brain Res. 1975;98(3):417-40.

73. Cuello AC, Bruno MA, Allard S, Leon W, Iulita MF. Cholinergic involvement in Alzheimer's disease. A link with NGF maturation and degradation. J Mol Neurosci. 2010;40(1-2):230-5. Epub 2009/08/15. eng.

74. Hotta H, Kagitani F, Kondo M, Uchida S. Basal forebrain stimulation induces NGF secretion in ipsilateral parietal cortex via nicotinic receptor activation in adult, but not aged rats. Neurosci Res. 2009;63(2):122-8. Epub 2008/12/09. eng.

75. Lacruz ME, Valentín A, Seoane JJG, Morris RG, Selway RP, Alarcón G. Single pulse electrical stimulation of the hippocampus is sufficient to impair human episodic memory. Neuroscience. 2010;170(2):623-32.

76. Cheney PD, Giffin DM, Van Acker GM. Neural Hijacking: Action of High-Frequency Electrical Stimulation on Cortical Circuits. The Neuroscientist. 2012;19(5):434-41.

77. Bartus RT, Dean RL, 3rd, Beer B, Lippa AS. The cholinergic hypothesis of geriatric memory dysfunction. Science. 1982;217(4558):408-14. Epub 1982/07/30. eng.

78. Nandi D, Jenkinson N, Stein J, Aziz T. The pedunculopontine nucleus in Parkinson's disease: primate studies. Br J Neurosurg. 2008;22 Suppl 1:S4-8. Epub 2008/12/23. eng.

79. Bohnen NI, Albin RL. The cholinergic system and Parkinson disease. Behav Brain Res. 2011;221(2):564-73. Epub 2010/01/12. eng.

80. Hardenacke K, Kuhn J, Lenartz D, Maarouf M, Mai J, Bartsch C, et al. Stimulate or degenerate Deep brain stimulation of the Nucleus basalis Meynert in Alzheimer's dementia. World Neurosurgery. 2013;(in press).

81. Hescham S, Lim LW, Jahanshahi A, Steinbusch HW, Prickaerts J, Blokland A, et al. Deep brain stimulation of the forniceal area enhances memory functions in experimental dementia: The role of stimulation parameters. Brain stimulation. 2012. Epub 2012/03/13. Eng.

82. Mu Y, Gage FH. Adult hippocampal neurogenesis and its role in Alzheimer's disease. Mol Neurodegener. 2011;6:85. Epub 2011/12/24. eng.

83. Fisher R, Salanova V, Witt T, Worth R, Henry T, Gross R, et al. Electrical stimulation of the anterior nucleus of thalamus for treatment of refractory epilepsy. Epilepsia. 2010;51(5):899-908. Epub 2010/03/25. eng.

84. Halgren E, Wilson CL, Stapleton JM. Human medial temporal-lobe stimulation disrupts both formation and retrieval of recent memories. Brain Cogn. 1985;4(3):287-95. Epub 1985/07/01. eng.

85. Lacruz ME, Valentin A, Seoane JJ, Morris RG, Selway RP, Alarcon G. Single pulse electrical stimulation of the hippocampus is sufficient to impair human episodic memory. Neuroscience. 2010;170(2):62332. Epub 2010/07/21. eng.

86. Voon V, Krack P, Lang AE, Lozano AM, Dujardin K, Schupbach M, et al. A multicentre study on suicide outcomes following subthalamic stimulation for Parkinson's disease. Brain. 2008;131(Pt 10):2720-8. Epub 2008/10/23. eng.

87. Harnack D, Meissner W, Jira JA, Winter C, Morgenstern R, Kupsch A. Placebo-controlled chronic high-frequency stimulation of the subthalamic nucleus preserves dopaminergic nigral neurons in a rat model of progressive Parkinsonism. Exp Neurol. 2008;210(1):257-60. Epub 2007/11/27. eng. 
88. Maesawa S, Kaneoke Y, Kajita Y, Usui N, Misawa N, Nakayama A, et al. Long-term stimulation of the subthalamic nucleus in hemiparkinsonian rats: neuroprotection of dopaminergic neurons. J Neurosurg. 2004;100(4):679-87. Epub 2004/04/09. eng.

89. Temel Y, Visser-Vandewalle V, Kaplan S, Kozan R, Daemen MA, Blokland A, et al. Protection of nigral cell death by bilateral subthalamic nucleus stimulation. Brain Res. 2006;1120(1):100-5. Epub 2006/09/27. eng.

90. Wallace BA, Ashkan K, Heise CE, Foote KD, Torres N, Mitrofanis J, et al. Survival of midbrain dopaminergic cells after lesion or deep brain stimulation of the subthalamic nucleus in MPTP-treated monkeys. Brain. 2007;130(Pt 8):2129-45. Epub 2007/06/23. eng.

91. Aggleton J, Keith A, Sahgal A. Both fornix and anterior thalamic, but not mammillary, lesions disrupt delayed non-matching-to-position memory in rats. Behav Brain Res. 1991;44(2):151-61.

92. Mumby DG, Piterkin P, Lecluse V, Lehmann H. Perirhinal cortex damage and anterograde objectrecognition in rats after long retention intervals. Behav Brain Res. 2007;185(2):82-7. 



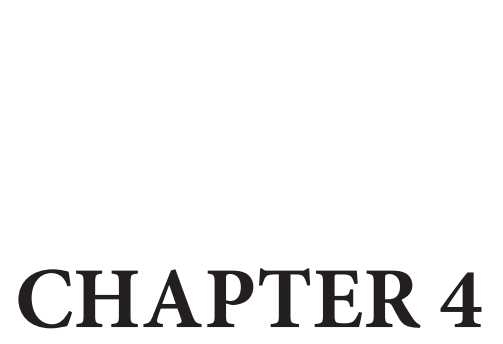

A NEUROANATOMICAL ANALYSIS

OF THE EFFECTS

OF A MEMORY IMPAIRING DOSE OF SCOPOLAMINE IN THE RAT BRAIN USING CYTOCHROME C OXIDASE AS PRINCIPLE MARKER

Sarah Hescham, Yasin Temel, João Casaca-Carreira, Kemal Arslantas, Youssef Yakkioui, Arjan Blokland, Ali Jahanshahi 


\section{ABSTRACT}

Acetylcholine plays a role in mnemonic and attentional processes, but also in locomotor and anxiety-related behavior. Receptor blockage by scopolamine can therefore induce cognitive as well as motor deficits and increase anxiety levels. Here we show that scopolamine, at a dose that has previously been found to affect learning and memory performance $(0.1 \mathrm{mg} / \mathrm{kg}$ i.p.), has a widespread effect on cytochrome c oxidase histochemistry in various regions of the rat brain. We found a down-regulation of cytochrome c oxidase in the nucleus basalis, in movement-related structures such as the primary motor cortex and the globus pallidus, memory-related structures such as the CA1 subfield of the hippocampus and perirhinal cortex and in the anxiety-related structures like the amygdala, which also plays a role in memory. However choline acetyltransferase levels were only affected in the CA1 subfield of the hippocampus and both, choline acetyltransferase and c-Fos expression levels were decreased in the amygdala. These findings corroborate strong cognitive behavioral effects of this drug, but also suggest possible anxiety and locomotor-related behavioral changes in subjects. Moreover, they present histochemical evidence that the effects of scopolamine are not ultimately restricted to cognitive parameters. 


\section{INTRODUCTION}

Acetylcholine has been implicated as a neurotransmitter involved in learning and memory. Therefore, a vast number of studies have demonstrated that the anticholinergic drug scopolamine produces cognitive deficits in both humans [1-4] and rodents [5-9]. Scopolamine is a non-selective muscarinic acetylcholine receptor antagonist [10], which binds the receptor with an affinity of $1 \mathrm{nM}$ in the rat brain [11]. There are five different muscarinic acetylcholine receptors, all of which are G-protein coupled: M1, M3 and M5 are linked to Gq, while M2 and M4 are autoreceptors linked to Gi/o [12]. Studies investigating the localization and distribution of these receptors have elucidated that M1 and M5 receptors in particular are located in the central nervous system, while M2, M3 and M4 are evenly distributed in the central and peripheral nervous system $[13,14]$. Due to its non-selective binding properties scopolamine has effects on anxiety levels, locomotor behavior and cognition $[15,16]$.

In general cholinergic neurons can be classified as projection neurons located mainly in the forebrain and upper brain stem, and interneurons, which are mainly found in the caudate-putamen, nucleus accumbens, hippocampus, cerebral cortex, hypothalamus and spinal cord. The basal forebrain cholinergic complex is composed of the septum, horizontal and vertical diagonal band of Broca, and nucleus basalis of Meynert. This complex provides the major cholinergic projections to the cerebral cortex and hippocampus [17]. In particular, neurons located in the medial septum predominantly innervate the hippocampus, while those of the vertical and horizontal diagonal band project to the anterior cingulate cortex and olfactory bulb, respectively [18]. The hippocampal formation and the medial prefrontal cortex play an important role in cognitive functioning. Cholinergic neurons of the nucleus basalis of Meynert provide efferents to the amygdala and habenular nuclei, both of which process memory and emotional reactions. Apart from this, primate and human pathological studies show that the nucleus basalis of Meynert also sends substantial efferent projections to a number of diencephalic structures, including the nucleus caudatus, putamen and thalamus, which are involved in motor behavior $[19,20]$. The pontine cholinergic nuclei, act mainly through thalamic intralaminar nuclei, but also through the reuniens nucleus and the anteriordorsal nucleus, and only provide minor innervation of the cerebral cortex [21].

Doses higher than $0.1 \mathrm{mg} / \mathrm{kg}$ of scopolamine have been shown to impair performances related to non-mnemonic processes, such as anxiety [22, 23]. A dose of $0.1 \mathrm{mg} /$ $\mathrm{kg}$ scopolamine, on the other side, appears to have only memory impairing properties as seen in various behavioral tasks in rodents, such as object location and recognition task, water maze and fear conditioning [6, 24-26]. Since this dose of scopolamine is used extensively in preclinical testing as a model evaluating new substances to treat memory dysfunction, we here provide the first overview of the histochemical effects in 
the rat brain when a cognitive impairing dose is administered. Hence, we can evaluate whether only cognitive regions are affected, or whether some side effects contribute to the drug-induced cognitive impairments. The present study used molecular markers of neural activity to test the hypothesis that the dose of $0.1 \mathrm{mg} / \mathrm{kg}$ has a widespread effect on the brain. The principle marker used was the endogenous metabolic enzyme cytochrome c oxidase (COX), supplemented by measurements of choline acetyltransferase (ChAT) and c-Fos (K-25) immunoreactivity. COX is an enzyme responsible for oxidative metabolism and therefore a marker for neural activity. Metabolic mapping is based on the principle that energy utilization in brain tissue is determined by the overall functional activity of neurons [27]. A decrease in COX levels can thus be associated with decreased neuronal activity at regional levels. C-Fos (K-25) can be used to map long-term neuronal activation at the cellular level, since it recognizes Fos, Fos-B, Fra-1 and Fra-2 $[28,29]$. We examined regions which are related to memory, emotions and motor behavior due to the various behavioral effects of scopolamine.

\section{MATERIALS AND METHODS}

\section{Subjects}

All procedures involving animals were carried out with approval of the Animal Experiments and Ethics Committee of Maastricht University. Adult male Sprague Dawley rats (body weight 300-350 g) from the Central Animal Facility (Maastricht University, the Netherlands) were housed in pairs in Makrolon cages, with rat chow and water available ad libitum. The colony room was maintained at a temperature of $21 \pm 1{ }^{\circ} \mathrm{C}$ and on a reversed 12:12 h light:dark cycle. Experimental manipulations were conducted during the dark phase under red light.

\section{Experimental groups}

Rats were randomly assigned to the following groups: saline $(\mathrm{n}=12)$ and scopolamine $(n=12)$.

\section{Drugs}

Scopolamine hydrobromide (Acros Organics BVBA, Geel, Belgium) was dissolved in vehicle (saline; $0.9 \% \mathrm{NaCl}$ ) and administered (i.p.) at a dose of $0.1 \mathrm{mg} / \mathrm{kg}$ (in $1 \mathrm{ml} /$ $\mathrm{kg}$ ) once daily over a time period of 5 days. This time period was chosen in order to resemble the time course of a behavioral task, in which scopolamine is administered repeatedly $[9,30,31]$. On the fifth day, rats were injected 60 min before decapitation or perfusion. 


\section{Histological analysis}

At the end of the experiment 6 rats of the saline group and 6 scopolamine treated animals were decapitated without anesthesia. Brains were rapidly removed, frozen in isopentane $\left(-45^{\circ} \mathrm{C}\right)$ and stored in $-80^{\circ} \mathrm{C}$. Subsequently, the brains were cut serially on a cryostat (Leica, Wetzlar, Germany) into $30 \mu \mathrm{m}$ coronal sections, thaw mounted on gelatine-coated slides and again stored at $-80^{\circ} \mathrm{C}$. To determine the metabolic activity of different brain structures, a cytochrome c oxidase (COX) staining was performed on the freshly frozen brain slides. For the COX staining, sections were incubated in a solution of Hepes buffer $(0.1 \mathrm{M})$ containing $4.5 \%$ sucrose, $0.12 \%$ 3,3'-diaminobenzidine$4 \mathrm{HCl}$ (DAB), $0.125 \%$ ammonium nickel sulfate and $0.023 \%$ cytochrome $\mathrm{C}$ type III (C2506, Sigma-Aldrich, Seelze, Germany), for the duration of 1 hour at $37^{\circ} \mathrm{C}$. To stop the reaction the tissue was fixated in a solution of neutral buffered formaldehyde for 10 minutes. Finally sections were rinsed, dehydrated, and cover slipped with depex mounting medium.

The remaining 6 saline treated and 6 scopolamine treated animals were transcardially perfused first with Tyrode $\left(\mathrm{NaCl}, \mathrm{KCl}, \mathrm{CaCl}_{2}, \mathrm{MgCl}_{2}, \mathrm{NaH}_{2} \mathrm{PO}_{4}, \mathrm{NaHCO}_{3}\right.$, glucose, distilled water) followed by Somogyi solution (4\% paraformaldehyde, 15\% picric acid, $0.05 \%$ glutaraldehyde in $0.1 \mathrm{M}$ phosphate buffered saline ( $\mathrm{pH} 7.6)$ ). To prevent the development of postperfusion artifacts, brains were fixed in fresh fixative (same content as in the Somogyi solution but lacking glutaraldehyde) at $4{ }^{\circ} \mathrm{C}$. After $2 \mathrm{~h}$, the brains were gently removed and stored in $1 \% \mathrm{NaN}_{3}$ in $4{ }^{\circ} \mathrm{C}$. Brains were embedded in $10 \%$ gelatin from porcine skin (Sigma-Aldrich, Zwyndrecht, The Netherlands), and cut into $30 \mu \mathrm{m}$ slices in the frontal plane using a vibratome (Leica ${ }^{\circledR}$, Wetzlar, Germany). Slices were immediately transferred into $1 \% \mathrm{NaN}_{3}$. Significant findings of the previous COX staining were further evaluated with ChAT and c-Fos (K-25) immunohistochemistry. In brief, sections were incubated overnight with polyclonal rabbit anti-c-fos (K-25) (1:2000; Santa Cruz Biotechnology Inc, Santa Cruz, USA) or choline acetyltransferase (H-95) (1:250; Santa Cruz Biotechnology Inc, Santa Cruz, USA) primary antibody and then, after washing, biotinylated donkey anti-rabbit secondary antibody (1:800; Jackson Immunoresearch Laboratories Inc., Westgrove, USA) followed by avidinbiotin peroxidase complex (1:800, Elite ABC-kit, Vestastatin, Burlingame, CA, USA). Both stainings were visualized by $\mathrm{DAB} / \mathrm{NiCl}_{2}$. Finally sections were mounted on glass, dehydrated, and cover slipped with pertex mounting medium.

Digital photos of COX, ChAT and c-Fos in the different regions were taken using the Cell P software (Olympus Soft Imaging Solutions, Münster, Germany) from a Olympus U-CMAD-2 digital camera connected to an Olympus AX 70 microscope (Olympus, Zoeterwoude, the Netherlands). For COX and ChAT densitometry (Image J software version 1.38x; NIH, Bethesda, USA) was performed for 2-3 antero-posterior levels of both right and left hemispheres. Histological data are presented as mean optical density 
(OD) ratio \pm S.E.M, which equals optical density/area of interest in $\mathrm{mm}^{2}$. Regions of interest were always delineated in their entirety, i.e. hippocampal subfields included pyramidal and dendritic layers. Cells immunopositive for c-Fos were counted manually, whereas the mean number of cells was corrected for surface area and expressed as cells/ $\mathrm{mm}^{2}$. Optical density measures of COX and ChAT were corrected by being subtracted from background measures of white matter. Bregma levels according to the rat brain atlas of Paxinos and Watson are as follows: prefrontal cortex $+3.72 \mathrm{~mm}$, primary and secondary motor cortex $+3.72 \mathrm{~mm}$, Ncl. Accumbens shell/core $+0.72 \mathrm{~mm}$, entorhinal and perirhinal cortex $-7.56 \mathrm{~mm}$, subthalamic nucleus $-3.36 \mathrm{~mm}$, hippocampus -3.36 $\mathrm{mm}$, lateral habenula $-3.36 \mathrm{~mm}$, amygdala $-2.16 \mathrm{~mm}$, globus pallidus and caudate putamen $-1.72 \mathrm{~mm}$, diagonal band of Broca and septum $+0.72 \mathrm{~mm}$, Ncl. Basalis -0.48 $\mathrm{mm}$, anterior thalamus $-1.92 \mathrm{~mm}$, dorsolateral periaqueductal gray (PAG) and dorsal raphe nucleus (DRN) $-7.56 \mathrm{~mm}$. As mentioned before, only significant histochemical findings of the COX staining were further investigated with ChAT and c-Fos (K-25) immunohistochemistry.

\section{Statistical analysis}

Optical densities and cell densities from the left and right hemispheres, as well as similar slides per structure were pooled and corrected individually for background. Slides containing cryodamage or other tissue damage were excluded in the analysis. An independent samples t-test was performed for the different targets and to supplement these findings partial eta-squared were individually reported as an estimate of the effect size. All p-values $\leq 0.05$ were considered significant.

\section{RESULTS}

Our findings showed a differential pattern of COX (de)activation in the brain (see Table 1). We found a significant downregulation of oxidative energy metabolism due to scopolamine injection in the following structures: CA1 (Fig.1 A and B), globus pallidus and nucleus basalis of Meynert (Fig. $1 \mathrm{C}$ and D), primary motor cortex (Fig. $1 \mathrm{E}$ and F), perirhinal cortex (Fig. $1 \mathrm{G}$ and $\mathrm{H}$ ) and amygdala (Fig. $1 \mathrm{I}$ and J). No effects of scopolamine treatment were found in these brain regions: diagonal band of Broca and medial septum (Fig. $1 \mathrm{~K}$ and L), secondary motor cortex, nucleus accumbens core and shell, caudate putamen, subthalamic nucleus, lateral habenula, dorsolateral PAG, prefrontal cortex, CA3, dentate gyrus, entorhinal cortex, anteriordorsal (AD) and anterioventralventral lateral (AVVL) thalamus and DRN.

ChAT was only downregulated in the CA1 $\left(\mathrm{t}(10)=2.724, \mathrm{p}=0.02, \eta^{2}=0.426\right.$; Fig. 2 $\mathrm{A}$ and $\mathrm{B})$ and the amygdala $\left(\mathrm{t}(10)=2.692, \mathrm{p}=0.02, \eta^{2}=0.420\right.$; Fig $2 \mathrm{C}$ and $\left.\mathrm{D}\right)$. No 
Table 1: Summary of cytochrome c oxidase optical density measures for different structures of the brain when comparing scopolamine to saline treated animals. Structures are organized according to their primary function and optical density measures are presented as mean (SEM). The statistical outcome includes the effect size (partial eta squared) and significant brain-depressant effects are presented in bold.

\begin{tabular}{|c|c|c|}
\hline Structure & Optical density & Statistical outcome \\
\hline \multirow[t]{2}{*}{ Diagonal band of Broca } & Saline: $776.8(37.3)$ & $\mathrm{t}(9)=0.296$, n.s., $\eta^{2}=0.010$ \\
\hline & Scopolamine: 754.1 (62.1) & \\
\hline \multirow[t]{2}{*}{ Ncl. Basalis } & Saline: $523.1(31.2)$ & $\mathbf{t}(9)=\mathbf{2 . 8 2 8}, \mathbf{p}=\mathbf{0 . 0 2 0}, \eta^{2}=0.470$ \\
\hline & Scopolamine: 311.3 (62.8) & \\
\hline \multirow[t]{2}{*}{ Medial Septum } & Saline: $248.4(63.0)$ & $\mathrm{t}(10)=1.065, \mathrm{n} . \mathrm{s}, \eta^{2}=0.102$ \\
\hline & Scopolamine: 165.9 (45.2) & \\
\hline \multirow[t]{2}{*}{ Primary Motor Cortex } & Saline: $472.7(42.4)$ & $\mathbf{t}(9)=3.314, p=0.009, \eta^{2}=0.550$ \\
\hline & Scopolamine: 300.2 (25.1) & \\
\hline \multirow[t]{2}{*}{ Secondary Motor Cortex } & Saline: $346.2(35.4)$ & $\mathrm{t}(8)=1.909$, n.s., $\eta^{2}=0.313$ \\
\hline & Scopolamine: 273.6 (13.8) & \\
\hline \multirow[t]{2}{*}{ Ncl. Accumbens Core } & Saline: $1036.5(122.8)$ & $\mathrm{t}(9)=2.084$, n.s., $\eta^{2}=0.325$ \\
\hline & Scopolamine: 658.8 (129.7) & \\
\hline \multirow[t]{2}{*}{ Ncl. Accumbens Shell } & Saline: $1373.8(235.1)$ & $\mathrm{t}(9)=1.616$, n.s., $\eta^{2}=0.225$ \\
\hline & Scopolamine: 917.5 (168.5) & \\
\hline \multirow[t]{2}{*}{ Caudate Putamen } & Saline: $848.9(88.9)$ & $\mathrm{t}(9)=1.275$, n.s., $\eta^{2}=0.153$ \\
\hline & Scopolamine: 714.6 (42.3) & \\
\hline \multirow[t]{2}{*}{ Globus Pallidus } & Saline: $288.0(14.8)$ & $\mathbf{t}(8)=4.457, p=0.002, \eta^{2}=0.713$ \\
\hline & Scopolamine: 152.5 (26.5) & \\
\hline \multirow[t]{2}{*}{ Subthalamic nucleus } & Saline: $797.0(97.3)$ & $\mathrm{t}(10)=1.878$, n.s., $\eta^{2}=0.261$ \\
\hline & Scopolamine: $521.2(110.0)$ & \\
\hline \multirow[t]{2}{*}{ Lateral Habenula } & Saline: $588.7(83.4)$ & $\mathrm{t}(10)=1.856$, n.s., $\eta^{2}=0.256$ \\
\hline & Scopolamine: 398.6 (59.5) & \\
\hline \multirow[t]{2}{*}{ Amygdala } & Saline: $313.3(22.8)$ & $t(10)=2.877, p=0.016, \eta^{2}=0.453$ \\
\hline & Scopolamine: 190.4 (36.1) & \\
\hline \multirow[t]{2}{*}{ Dorsal Raphe Nucleus } & Saline: $1024.9(100.8)$ & $\mathrm{t}(10)=0.506$, n.s., $\eta^{2}=0.025$ \\
\hline & Scopolamine: 957.6 (86.6) & \\
\hline \multirow[t]{2}{*}{ Dorsolateral PAG } & Saline: 793.5 (48.7) & $\mathrm{t}(10)=1.561$, n.s., $\eta^{2}=0.196$ \\
\hline & Scopolamine: 699.7 (35.3) & \\
\hline \multirow[t]{2}{*}{ Prelimbic Cortex } & Saline: 436.7 (64.5) & $\mathrm{t}(9)=1.140$, n.s., $\eta^{2}=0.126$ \\
\hline & Scopolamine: 350.6 (27.6) & \\
\hline \multirow[t]{2}{*}{ Infralimbic Cortex } & Saline: $335.8(69.5)$ & $\mathrm{t}(9)=1.296$, n.s., $\eta^{2}=0.157$ \\
\hline & Scopolamine: 233.3 (20.5) & \\
\hline \multirow[t]{2}{*}{ Cingulate Gyrus } & Saline: $424.7(52.4)$ & $\mathrm{t}(9)=0.765$, n.s., $\eta^{2}=0.061$ \\
\hline & Scopolamine: 376.3 (28.1) & \\
\hline \multirow[t]{2}{*}{ CA1 } & Saline: $939.1(25.2)$ & $\mathbf{t}(9)=\mathbf{2 . 3 2 3}, \mathbf{p}=\mathbf{0 . 0 4 5}, \eta^{2}=0.375$ \\
\hline & Scopolamine: 791.2 (53.8) & \\
\hline \multirow[t]{2}{*}{ CA3 } & Saline: $776.3(96.7)$ & $\mathrm{t}(9)=1.458$, n.s., $\eta^{2}=0.191$ \\
\hline & Scopolamine: 607.4 (69.0) & \\
\hline \multirow[t]{2}{*}{ Dentate Gyrus } & Saline: $2196.1(112.7)$ & $\mathrm{t}(8)=0.564$, n.s., $\eta^{2}=0.038$ \\
\hline & Scopolamine: $2263.2(38.3)$ & \\
\hline \multirow[t]{2}{*}{ Entorhinal cortex } & Saline: $377.0(21.0)$ & $\mathrm{t}(10)=1.832$, n.s., $\eta^{2}=0.251$ \\
\hline & Scopolamine: 289.6 (42.9) & \\
\hline \multirow[t]{2}{*}{ Perirhinal cortex } & Saline: $451.0(20.8)$ & $\mathbf{t}(10)=2.358, p=0.040, \eta^{2}=0.357$ \\
\hline & Scopolamine: 342.6 (41.0) & \\
\hline \multirow[t]{2}{*}{ AD Thalamus } & Saline: $2453.0(144.7)$ & $\mathrm{t}(8)=1.158$, n.s., $\eta^{2}=0.144$ \\
\hline & Scopolamine: 2681.6 (99.8) & \\
\hline AVVL Thalamus & Saline: $1723.8(152.6)$ & $\mathrm{t}(10)=0.933$, n.s., $\eta^{2}=0.080$ \\
\hline & Scopolamine: 1501.1 (183.6) & \\
\hline
\end{tabular}


changes were found in the nucleus basalis, primary motor cortex, globus pallidus and perirhinal cortex (Fig. $2 \mathrm{E}$ ). C-Fos expression was downregulated only in the amygdala $\left(t(9)=3.860, p=0.004, \eta^{2}=0.623\right.$; Fig. $3 \mathrm{C}$ and $\left.\mathrm{D}\right)$, while the expression of $\mathrm{c}$-Fos in the CA1 subfield of the hippocampus (Fig. $3 \mathrm{~A}$ and B), nucleus basalis, primary motor cortex, globus pallidus and perirhinal cortex did not differ from control animals (Fig. 3 E).

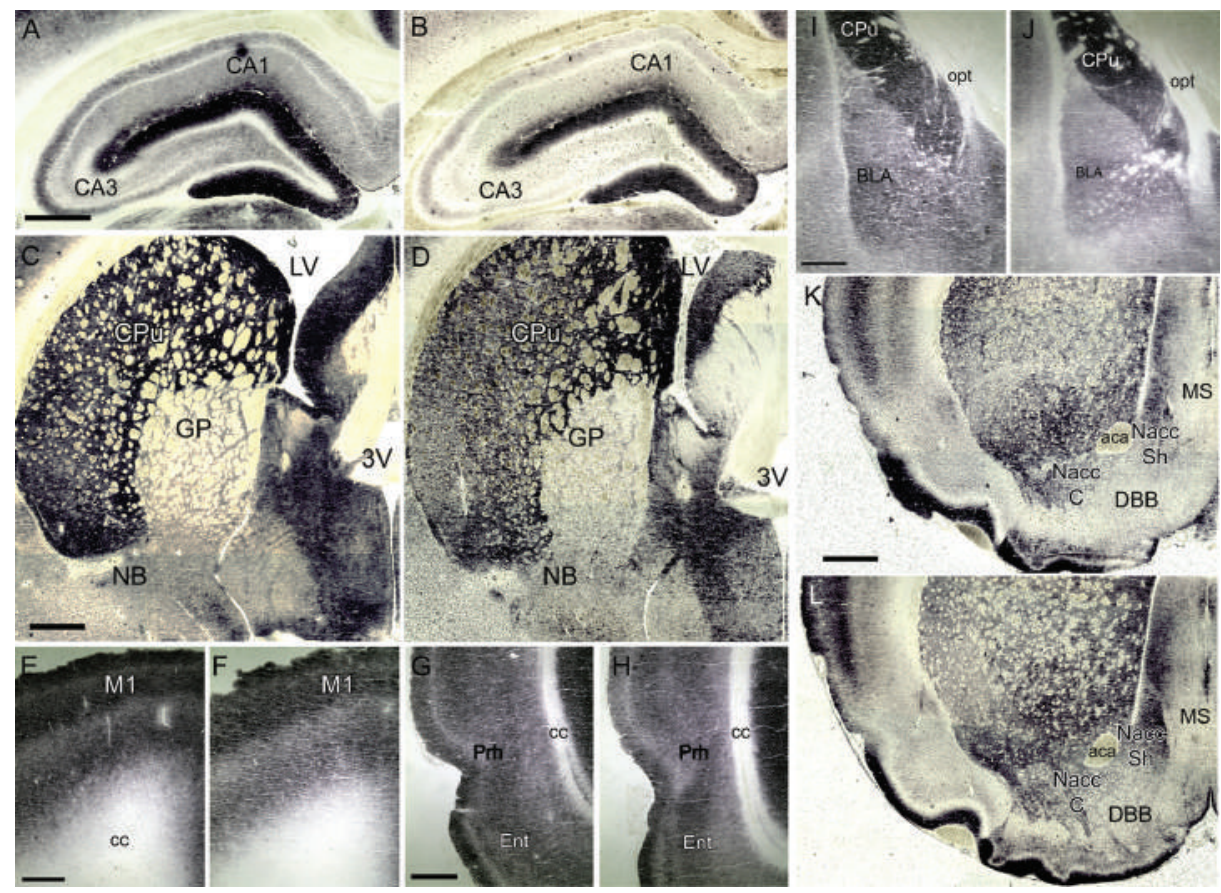

Figure 1: Representative low-power photomicrographs of coronal brain sections stained for COX showing the hippocampus ( $\mathrm{A}$ and $\mathrm{B}$ ), globus pallidus $(\mathrm{C}$ and $\mathrm{D})$, primary motor cortex (E and $\mathrm{F}$ ), perirhinal cortex ( $\mathrm{G}$ and $\mathrm{H}$ ), amygdala (I and J) and diagonal band of Broca (K and L) of saline (left) and scopolamine (right) treated rats. Anatomical levels are according to the rat brain atlas of Paxinos and Watson (1998). (A and B) Hippocampus CA1 and CA3 at Bregma $-3.36 \mathrm{~mm}$. Scale bar $=1000 \mu \mathrm{m}$. (C and D) Globus pallidus (GP), caudate putamen $(\mathrm{CPu})$ and nucleus basalis $(\mathrm{NB})$ at Bregma $-0.48 \mathrm{~mm}$. Scale bar $=1000 \mu \mathrm{m}$. (E and F) Primary motor cortex (M1) at Bregma $+3.72 \mathrm{~mm}$. Scale bar $=500 \mu \mathrm{m}$. (G and H) Perirhinal (Prh) and entorhinal (Ent) cortex at Bregma $-7.56 \mathrm{~mm}$. Scale bar $=500 \mu \mathrm{m}$. (I and J) Amygdala (BLA) at Bregma $-2.16 \mathrm{~mm}$. Scale bar $=500 \mu \mathrm{m}$. (K and L) Diagonal Band (DBB), medial septum (MS) and nucleus accumbens core (NAcc C) and shell (NAcc Sh) at Bregma $+0.72 \mathrm{~mm}$. Scale bar $=1000 \mu \mathrm{m}$. 


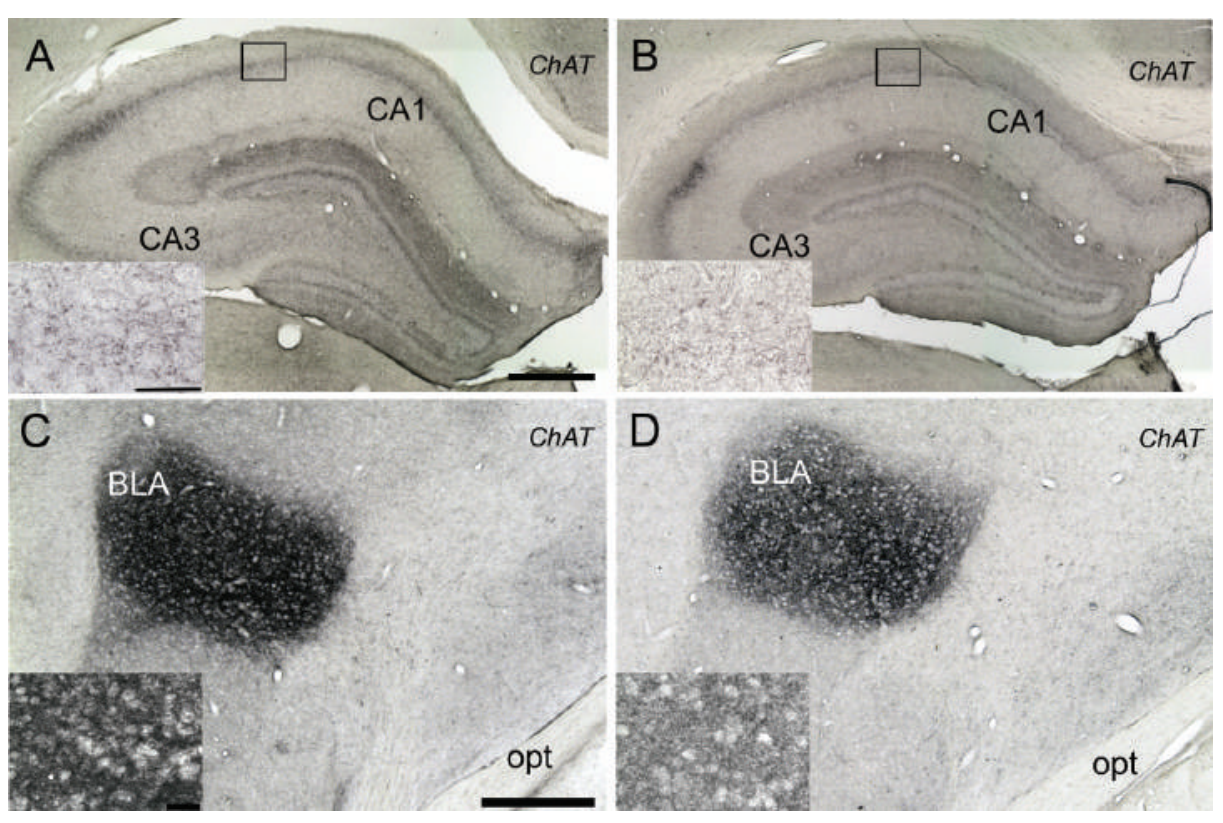

Figure 2: Representative low-power photomicrographs of coronal brain sections stained for choline acetyltransferase (ChAT) showing the hippocampus (A and B) and the amygdala (C and D) of saline (left) and scopolamine (right) treated rats. The high-power photomicrograph insets in the lower left corner show the CA1 subregion of the hippocampus and the amygdala, respectively. (A and B) Hippocampus CA1 and CA3 at Bregma _3.36 mm. Scale bar $=500 \mathrm{~mm}$, scale bar of inset $=50 \mathrm{~mm}$. (C and D) Amygdala (BLA) at Bregma $-2.16 \mathrm{~mm}$. Scale bar $=500 \mu \mathrm{m}$, scale bar of inset $=50 \mu \mathrm{m}$.

\section{DISCUSSION}

Cholinergic neurotransmission is essential for many forms of learning and memory. It has been claimed that the anticholinergic action of scopolamine may more readily impact the hippocampus than other structures [25, 32-34]. Here we provide histochemical and immunohistochemical evidence that both COX and ChAT are down-regulated in the CA1 region of the hippocampus and the amygdala after chronic scopolamine treatment. With c-Fos (K-25) we only found a significant decrease in Fos positive cells in the amygdala and no difference in the CA1 region of the hippocampus between scopolamine and saline injected animals.

The hippocampus and the amygdala both belong to the limbic system and are linked to two independent memory systems, which constantly interact with each other [35]. The hippocampus is critical for episodic memory and correlates with subsequent memory for neutral items. However, variables such as context and prior experience affect the way in which information is retained and the accuracy with which the retention occurs. In fact, the amygdala can modulate these memories through emotional 


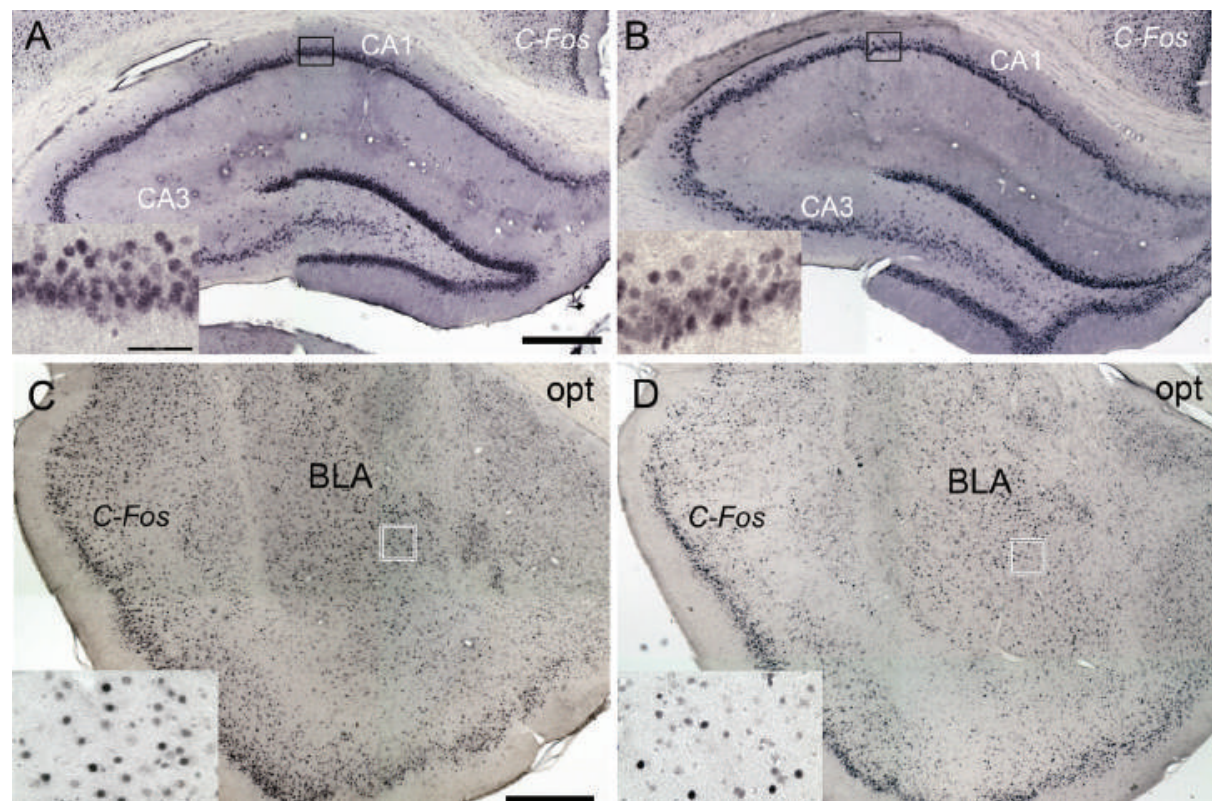

Figure 3: Representative low-power photomicrographs of coronal brain sections stained for c-Fos showing the hippocampus (A and B) and the amygdala (C and D) of saline (left) and scopolamine (right) treated rats. The high-power photomicrograph insets in the lower left corner show the CA1 subregion of the hippocampus and the amygdala, respectively. (A and B) Hippocampus CA1 and CA3 at Bregma $-3.36 \mathrm{~mm}$. Scale bar $=500 \mu \mathrm{m}$, scale bar of inset $=50 \mu \mathrm{m}$. (C and D) Amygdala (BLA) at Bregma -2.16 $\mu \mathrm{m}$. Scale bar $=500 \mu \mathrm{m}$, scale bar of inset $=50 \mu \mathrm{m}$.
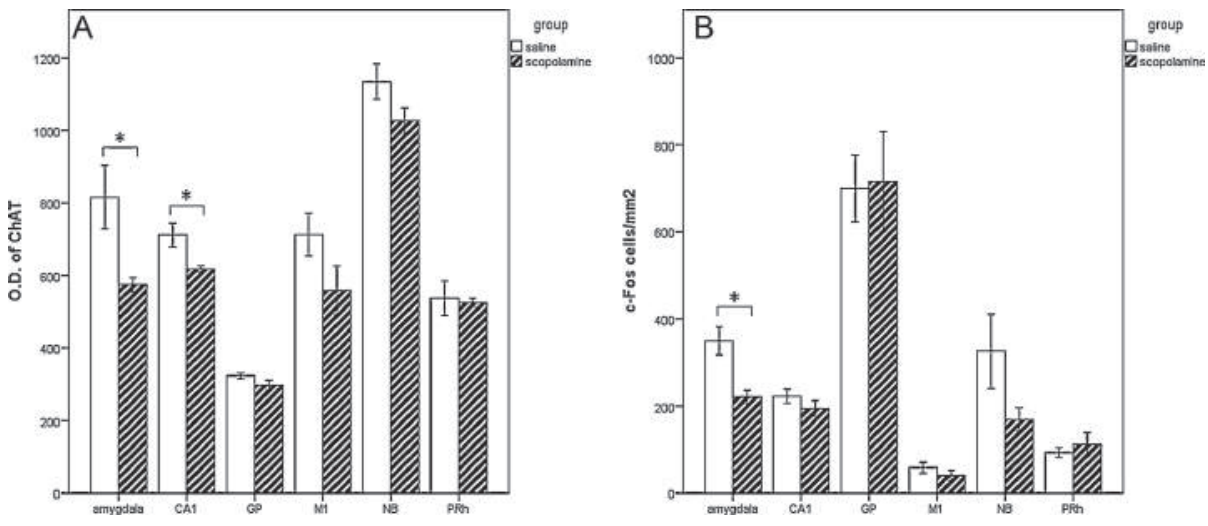

Figure 4: Cumulative data showing the means \pm SEM of (A) choline acetyltransferase (ChAT) optical density levels and (B) c-Fos expression, measured in the amygdala, CA1 subregion of the hippocampus, globus pallidus (GP), primary motor cortex (M1), nucleus basalis (NB) and perirhinal cortex (Prh). Note that, scopolamine treated animals have decreased ChAT activity in the amygdala and CA1. C-Fos cells were only declined in the amygdala of the scopolamine treated animals. Statistically significant difference $(\mathrm{P}<0.05)$ is indicated by an $\left(^{*}\right)$. 
responses. Thus, damage to the amygdala results in a profound impairment of learning abilities in tasks that require the subject to make a connection between environmental stimuli and strong emotional responses [36]. The down-regulation of c-Fos (K-25), ChAT and COX levels in the amygdala could explain the effects of scopolamine on impaired context and cued conditioning [15]. Future studies should investigate structures belonging to the major pathway mediating emotional/anxiety-related effects on memory in more detail.

With the dose of $0.1 \mathrm{mg} / \mathrm{kg}$ scopolamine there was no effect on ChAT and c-Fos levels in the motor and basal forebrain structures, suggesting that the aforementioned dose only acts on the memory system. However, the effects of scopolamine at the dose applied might have been too subtle to be detected by immunohistochemistry in these brain regions.

Using a different approach, namely COX histochemistry, we were able to detect additional effects on the brain of the relatively low dose of scopolamine. We showed a significant down-regulation of COX activity with an injection dose of $0.1 \mathrm{mg} / \mathrm{kg} \mathrm{sco-}$ polamine in the CA1 subregion of the hippocampus. However, this was not found for the CA3 subfield or dentate gyrus. It has been shown before, that M1and M4 receptors are enriched in the CA1 region when compared to the CA3 [37].The CA3 subfield of the hippocampus rather contains more $\mathrm{M} 2$ receptors, while $\mathrm{m} 1-\mathrm{m} 4$ immunoreactivities are deferentially localized in neuronal populations and neuropil in the dentate gyrus [37]. We also found a clear reduced COX level in the perirhinal, but not the entorhinal cortex. The anteriodorsal and anterioventral nuclei of the thalamus have also been implicated in cognitive functions. In these regions mainly M3 receptor subtypes are expressed [38]. However, scopolamine did not affect the COX level in the anteriodorsal and anterioventral nuclei of the thalamus.

Since scopolamine has effects on anxiety related behavior we examined some regions that are implicated in this behavioral domain. As mentioned before, we found a decrease of COX activity in the amygdala, but no effects in the periaqueductal gray. The periaqueductal gray contains predominantly M1 and M2 receptors. It has been found that activation of M1 and M2 receptor subtypes usually inhibit GABAergic and glutamaterig synaptic transmission in this structure [39]. Furthermore, there were no effects on the lateral habenular nuclei, which contain high levels of M3 receptor subtypes.

Besides, we found a strong COX down-regulation in the nucleus basalis but not in the medial septum or diagonal band of Broca. This implies that at a dose of $0.1 \mathrm{mg} / \mathrm{kg}$ the effects of scopolamine are mainly mediated via nucleus basalis and less likely via the medial septum or diagonal band of Broca. Birthelmer and colleagues have also suggested that acetylcholine from the medial septum and the diagonal band is not derived from intrinsic neurons but rather from the lateral septum or from terminals of neurons located outside the septal region [40]. Another possibility might include compensation 
mechanisms. Inactivation or lesion of neurons in the medial septum-diagonal band of Broca completely abolishes theta oscillation in the hippocampus, demonstrating a critical role of these two structures [41]. In fact, the median raphe nucleus projects the efferent serotonergic pathway to $\gamma$-aminobutyric acid (GABA) interneurons of the medial septum - diagonal band complex, which suppresses the generation of hippocampal theta waves [41-43]. Therefore the medial septum and the diagonal band possess more complex functions besides cholinergic transmission.

We did not find any significant changes in COX levels in the medial prefrontal cortex, i.e. the cingulate gyrus, prelimbic and infralimbic cortex. Therefore, we can conclude that the injection dose of $0.1 \mathrm{mg} / \mathrm{kg}$ scopolamine does not affect medial prefrontal cortex activity. The primary motor cortex, however, showed decreased COX activity when compared to the secondary motor cortex. Scopolamine also had clear effects on COX levels of the globus pallidus, a structure mainly involved in motor functions. Within the striatum and accumbens, mainly M2 receptors and to a lesser extend M1 and M4 receptors are expressed [44, 45]. COX activity was unaffected in regions like the caudate putamen, STN, nucleus accumbens core and shell. The reduced COX activity in some of the motor regions supports previous studies showing decreased locomotor activity after scopolamine treatment [5, 46-48]. It should be noted that a few studies have reported no effects or an increase in motor activity after scopolamine injections $[15,49]$. This may at least be explained by differences in experimental factors such as strain, test and dose.

Since scopolamine was found to reduce COX activity in different regions it implies that scopolamine decreases neuronal activity in various structures of the brain. Of note, there were no brain regions with an increased COX activity.

Several muscarinic receptor subtypes might underlie the cognitive effects of scopolamine. However, muscarinic receptor subtypes are also present in brain structures not primarily involved in learning and memory, but locomotion and anxiety. Muscarinic receptors can furthermore be found in the periphery. In this study we showed that chronic scopolamine administration indeed has a widespread effect on brain depressant activity which corroborates the variety of behavioral effects of this drug (e.g., anxiety, motor activity). We have, however, not investigated peripheral effects of scopolamine, nor can we speculate whether these results would be different following an acute administration paradigm, although cognitive impairments are prominent after a single administration. Due to its non-selective binding properties and its widespread effects on behavior, scopolamine may not be an ideal drug to induce cognitive deficits. 


\section{ACKNOWLEDGEMENTS}

This study was supported by the Internationale Stichting Alzheimer Onderzoek (ISAO). 


\section{REFERENCES}

1. Ebert, Kirch. Scopolamine model of dementia: electroencephalogram findings and cognitive performance. European Journal of Clinical Investigation. 1998;28(11):944-9.

2. Flicker C, Ferris SH, Serby M. Hypersensitivity to scopolamine in the elderly. Psychopharmacology (Berl). 1992;107(2-3):437-41. Epub 1992/01/01. eng.

3. Sherman SJ, Atri A, Hasselmo ME, Stern CE, Howard MW. Scopolamine impairs human recognition memory: data and modeling. Behav Neurosci. 2003;117(3):526-39. Epub 2003/06/14. eng.

4. Richardson JS, Miller PS, Lemay JS, Jyu CA, Neil SG, Kilduff CJ, et al. Mental dysfunction and the blockade of muscarinic receptors in the brains of the normal elderly. Prog Neuropsychopharmacol Biol Psychiatry. 1985;9(5-6):651-4. Epub 1985/01/01. eng.

5. Hodges DB, Jr., Lindner MD, Hogan JB, Jones KM, Markus EJ. Scopolamine induced deficits in a battery of rat cognitive tests: comparisons of sensitivity and specificity. Behav Pharmacol. 2009;20(3):237-51. Epub 2009/05/14. eng.

6. Sambeth A, Riedel WJ, Smits LT, Blokland A. Cholinergic drugs affect novel object recognition in rats: relation with hippocampal EEG? Eur J Pharmacol. 2007;572(2-3):151-9. Epub 2007/07/31. eng.

7. Barak S, Weiner I. Towards an animal model of an antipsychotic drug-resistant cognitive impairment in schizophrenia: scopolamine induces abnormally persistent latent inhibition, which can be reversed by cognitive enhancers but not by antipsychotic drugs. Int J Neuropsychopharmacol. 2009;12(2):227-41. Epub 2008/08/09. eng.

8. Buccafusco JJ, Terry AV, Jr., Webster SJ, Martin D, Hohnadel EJ, Bouchard KA, et al. The scopolaminereversal paradigm in rats and monkeys: the importance of computer-assisted operant-conditioning memory tasks for screening drug candidates. Psychopharmacology (Berl). 2008;199(3):481-94. Epub 2007/07/28. eng.

9. Janas AM, Cunningham SC, Duffy KB, Devan BD, Greig NH, Holloway HW, et al. The cholinesterase inhibitor, phenserine, improves Morris water maze performance of scopolamine-treated rats. Life Sciences. 2005;76(10):1073-81.

10. Bolden C, Cusack B, Richelson E. Antagonism by antimuscarinic and neuroleptic compounds at the five cloned human muscarinic cholinergic receptors expressed in Chinese hamster ovary cells. The Journal of pharmacology and experimental therapeutics. 1992;260(2):576-80. Epub 1992/02/01. eng.

11. Lee JH, el-Fakahany EE. Heterogeneity of binding of muscarinic receptor antagonists in rat brain homogenates. The Journal of pharmacology and experimental therapeutics. 1985;233(3):707-14. Epub 1985/06/01. eng.

12. Conn PJ, Jones CK, Lindsley CW. Subtype-selective allosteric modulators of muscarinic receptors for the treatment of CNS disorders. Trends in pharmacological sciences. 2009;30(3):148-55. Epub 2009/02/10. eng.

13. Caulfield MP. Muscarinic receptors-characterization, coupling and function. Pharmacology \& therapeutics. 1993;58(3):319-79. Epub 1993/06/01. eng.

14. Van der Zee EA, Keijser JN. Localization of pre- and postsynaptic cholinergic markers in rodent forebrain: a brief history and comparison of rat and mouse. Behavioural brain research. 2011;221(2):35666. Epub 2010/12/07. eng.

15. Klinkenberg I, Blokland A. The validity of scopolamine as a pharmacological model for cognitive impairment: a review of animal behavioral studies. Neurosci Biobehav Rev. 2010;34(8):1307-50. Epub 2010/04/20. eng. 
16. Hughes RN, Otto MT. Anxiolytic effects of environmental enrichment attenuate sex-related anxiogenic effects of scopolamine in rats. Prog Neuropsychopharmacol Biol Psychiatry. 2013;40:252-9. Epub 2012/10/23. eng.

17. Schliebs R, Arendt T. The cholinergic system in aging and neuronal degeneration. Behavioural Brain Research. 2011;221(2):555-63.

18. Schliebs R, Arendt T. The significance of the cholinergic system in the brain during aging and in Alzheimer's disease. J Neural Transm. 2006;113(11):1625-44. English.

19. Mesulam MM, Mufson EJ, Levey AI, Wainer BH. Cholinergic innervation of cortex by the basal forebrain: cytochemistry and cortical connections of the septal area, diagonal band nuclei, nucleus basalis (substantia innominata), and hypothalamus in the rhesus monkey. J Comp Neurol. 1983;214(2):17097. Epub 1983/02/20. eng.

20. Mesulam MM, Geula C. Nucleus basalis (Ch4) and cortical cholinergic innervation in the human brain: observations based on the distribution of acetylcholinesterase and choline acetyltransferase. J Comp Neurol. 1988;275(2):216-40. Epub 1988/09/08. eng.

21. Mesulam MM, Mufson EJ, Wainer BH, Levey AI. Central cholinergic pathways in the rat: an overview based on an alternative nomenclature (Ch1-Ch6). Neuroscience. 1983;10(4):1185-201. Epub 1983/12/01. eng.

22. Hughes RN, Desmond CS, Fisher LC. Room novelty, sex, scopolamine and their interactions as determinants of general activity and rearing, and light-dark preferences in rats. Behav Processes. 2004;67(2):173-81. Epub 2004/07/09. eng.

23. Smythe JW, Bhatnagar S, Murphy D, Timothy C, Costall B. The effects of intrahippocampal scopolamine infusions on anxiety in rats as measured by the black-white box test. Brain Res Bull. 1998;45(1):89-93. Epub 1998/01/20. eng.

24. Hescham S, Lim LW, Jahanshahi A, Steinbusch HW, Prickaerts J, Blokland A, et al. Deep brain stimulation of the forniceal area enhances memory functions in experimental dementia: the role of stimulation parameters. Brain Stimul. 2013;6(1):72-7. Epub 2012/03/13. eng.

25. Anagnostaras SG, Maren S, Sage JR, Goodrich S, Fanselow MS. Scopolamine and Pavlovian fear conditioning in rats: dose-effect analysis. Neuropsychopharmacology. 1999;21(6):731-44. Epub 2000/01/14. eng.

26. Buresova O, Bolhuis JJ, Bures J. Differential effects of cholinergic blockade on performance of rats in the water tank navigation task and in a radial water maze. Behav Neurosci. 1986;100(4):476-82. Epub 1986/08/01. eng.

27. Wong-Riley MT. Cytochrome oxidase: an endogenous metabolic marker for neuronal activity. Trends in neurosciences. 1989;12(3):94-101. Epub 1989/03/01. eng.

28. Budzikowski AS, Vahid-Ansari F, Leenen FH. Chronic activation of brain areas by high-sodium diet in Dahl salt-sensitive rats. Am J Physiol. 1998;274(6 Pt 2):H2046-52. Epub 1998/06/25. eng.

29. Jahanshahi A, Schonfeld L, Janssen ML, Hescham S, Kocabicak E, Steinbusch HW, et al. Electrical stimulation of the motor cortex enhances progenitor cell migration in the adult rat brain. Exp Brain Res. 2013. Epub 2013/09/05. Eng.

30. Chalas A, Conway EL. No evidence for involvement of angiotensin II in spatial learning in water maze in rats. Behavioural brain research. 1996;81(1-2):199-205.

31. Hescham S, Lim LW, Jahanshahi A, Steinbusch HW, Prickaerts J, Blokland A, et al. Deep brain stimulation of the forniceal area enhances memory functions in experimental dementia: the role of stimulation parameters. Brain Stimul. 2013;6(1):72-7.

32. Rogers JL, Kesner RP. Cholinergic modulation of the hippocampus during encoding and retrieval. Neurobiol Learn Mem. 2003;80(3):332-42. Epub 2003/10/03. eng. 
33. Watts J, Stevens R, Robinson C. Effects of scopolamine on radial maze performance in rats. Physiol Behav. 1981;26(5):845-51. Epub 1981/05/01. eng.

34. Gale GD, Anagnostaras SG, Fanselow MS. Cholinergic modulation of pavlovian fear conditioning: effects of intrahippocampal scopolamine infusion. Hippocampus. 2001;11(4):371-6. Epub 2001/09/04. eng.

35. Phelps EA. Human emotion and memory: interactions of the amygdala and hippocampal complex. Curr Opin Neurobiol. 2004;14(2):198-202. Epub 2004/04/15. eng.

36. Richter-Levin G, Akirav I. Amygdala-hippocampus dynamic interaction in relation to memory. Mol Neurobiol. 2000;22(1-3):11-20. Epub 2001/06/21. eng.

37. Levey AI, Edmunds SM, Koliatsos V, Wiley RG, Heilman CJ. Expression of m1-m4 muscarinic acetylcholine receptor proteins in rat hippocampus and regulation by cholinergic innervation. J Neurosci. 1995;15(5 Pt 2):4077-92. Epub 1995/05/01. eng.

38. Levey AI, Edmunds SM, Heilman CJ, Desmond TJ, Frey KA. Localization of muscarinic m3 receptor protein and M3 receptor binding in rat brain. Neuroscience. 1994;63(1):207-21. Epub 1994/11/01. eng.

39. Lau BK, Vaughan CW. Muscarinic modulation of synaptic transmission via endocannabinoid signalling in the rat midbrain periaqueductal gray. Molecular pharmacology. 2008;74(5):1392-8. Epub 2008/08/06. eng.

40. Birthelmer A, Lazaris A, Riegert C, Marques Pereira P, Koenig J, Jeltsch H, et al. Does the release of acetylcholine in septal slices originate from intrinsic cholinergic neurons bearing p $75 \mathrm{ntr}$ receptors? a study using 192 IgG-saporin lesions in rats. Neuroscience. 2003;122(4):1059-71.

41. Yoder RM, Pang KC. Involvement of GABAergic and cholinergic medial septal neurons in hippocampal theta rhythm. Hippocampus. 2005;15(3):381-92. Epub 2005/01/05. eng.

42. Alreja M. Excitatory actions of serotonin on GABAergic neurons of the medial septum and diagonal band of Broca. Synapse. 1996;22(1):15-27. Epub 1996/01/01. eng.

43. Leranth C, Vertes RP. Median raphe serotonergic innervation of medial septum/diagonal band of broca (MSDB) parvalbumin-containing neurons: possible involvement of the MSDB in the desynchronization of the hippocampal EEG. J Comp Neurol. 1999;410(4):586-98. Epub 1999/07/09. eng.

44. Weiner DM, Levey AI, Brann MR. Expression of muscarinic acetylcholine and dopamine receptor mRNAs in rat basal ganglia. Proceedings of the National Academy of Sciences of the United States of America. 1990;87(18):7050-4. Epub 1990/09/01. eng.

45. Yee J, Famous KR, Hopkins TJ, McMullen MC, Pierce RC, Schmidt HD. Muscarinic acetylcholine receptors in the nucleus accumbens core and shell contribute to cocaine priming-induced reinstatement of drug seeking. Eur J Pharmacol. 2011;650(2-3):596-604. Epub 2010/11/03. eng.

46. Falsafi SK, Deli A, Hoger H, Pollak A, Lubec G. Scopolamine administration modulates muscarinic, nicotinic and NMDA receptor systems. PloS one. 2012;7(2):e32082. Epub 2012/03/03. eng.

47. Masuoka T, Fujii Y, Kamei C. Effect of scopolamine on the hippocampal theta rhythm during an eight-arm radial maze task in rats. Eur J Pharmacol. 2006;539(1-2):76-80. Epub 2006/05/10. eng.

48. Myhrer T, Enger S, Aas P. Cognitive side effects in rats caused by pharmacological agents used to prevent soman-induced lethality. Eur J Pharmacol. 2004;483(2-3):271-9.

49. Humby T, Laird FM, Davies W, Wilkinson LS. Visuospatial attentional functioning in mice: interactions between cholinergic manipulations and genotype. The European journal of neuroscience. 1999;11(8):2813-23. Epub 1999/08/24. eng. 




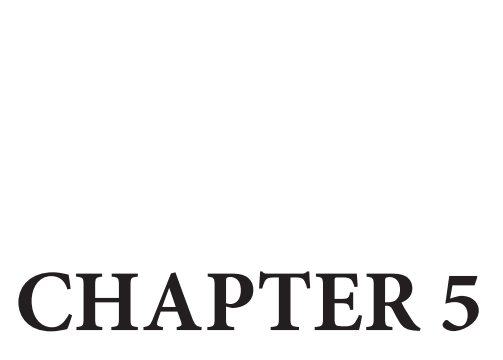

DEEP BRAIN STIMULATION OF THE FORNICEAL AREA ENHANCES MEMORY FUNCTIONS IN EXPERIMENTAL DEMENTIA: THE ROLE OF STIMULATION

\section{PARAMETERS}

Sarah Hescham, LeeWei Lim, Ali Jahanshahi, Harry

Steinbusch, Jos Prickaerts, Arjan Blokland, Yasin Temel 


\section{ABSTRACT}

Deep brain stimulation (DBS) is currently being evaluated as a potential therapy in improving memory functions in Alzheimer's disease. The target for DBS and the stimulation parameters to be used are unknown. Here, we implanted bilateral electrodes in the vicinity of the fornix, a key element of the memory circuitry, and applied DBS with different stimulation frequencies and amplitudes in an experimental model of dementia. Rats received scopolamine, a muscarinic acetylcholine receptor antagonist, to mimic memory impairment. Rats were then tested in the object location task with the following conditions: (i) with attachment of stimulation cable (off stimulation), and (ii) with DBS at various amplitudes $(50 \mu \mathrm{A}, 100 \mu \mathrm{A}$ and $200 \mu \mathrm{A}), 100 \mu$ s pulse width and $100 \mathrm{~Hz}$ or $10 \mathrm{~Hz}$ stimulation frequency. DBS reversed the memory impairing effects of scopolamine when compared to sham rats. We found that the fornix is not sensitive to the frequency of stimulation, but rather to current levels. With the most optimal stimulation parameter, we found no side-effects on anxiety levels and general motor activity. These findings identify the fornix as a key region in controlling spatial memory functions. DBS of this region, using tailored stimulation parameters, has the potential to improve memory functions in conditions characterised by memory impairment. 


\section{INTRODUCTION}

In the past years, the applicability of deep brain stimulation (DBS) in psychiatry has been evaluated in disorders of mood and thought. DBS of 'key' regions within the limbic system resulted in therapeutic effects in patients with treatment-resistant depression [1-3] and obsessive-compulsive disorder [4]. In this respect, recent clinical studies have suggested that DBS can be used as a tool to enhance memory functions. For instance, Hamani and colleagues [5] stimulated the fornix/hypothalamus area in a patient suffering from morbid obesity. Remarkably, DBS in this patient generated detailed autobiographical memories. Electroencephalographic source localization elucidated that DBS evoked activity in medial temporal lobe structures like the hippocampus. Because of this case-observation, the same group performed the first study in patients with Alzheimer's disease (AD) [6]. Six patients with mild AD were implanted with electrodes in the area of the fornix/hypothalamus and after an intraoperative evaluation of stimulation, patients received chronic DBS at 3 to 3.5 Volts with the frequency set at $130 \mathrm{~Hz}$ and the pulse width at $90 \mu$ s for a period of 12 months. Although being a safety study, the central hypothesis was to use DBS to drive the activity of the fornix and obtain beneficial effects on memory functions. Indeed, the authors have found that the application of DBS in the hypothalamus/fornix vicinity was safe and triggered neural activity in the memory circuit, including the entorhinal and hippocampal areas. PET scans showed an early and striking reversal of the impaired glucose utilization in the temporal and parietal lobes that was maintained after 12 months of continuous stimulation. Evaluation of the AD Assessment Scale cognitive subscale and the Mini Mental State Examination suggested possible improvements and/or slowing in the rate of cognitive decline at 6 and 12 months, especially in patients that were less severely affected at the time of surgery.

The fornix is part of the so-called circuit of Papez. The circuit of Papez is one of the major pathways of the limbic system and is primarily involved in the cortical control of emotions and in storing memory [7]. Information is considered to circulate within the circuit of Papez for a certain time and is strongly linked with internal states (emotional as well as motivational) before being processed for long-term storage. In this circuit, the entorhinal cortex projects to the hippocampus, whose efferents are bundled in the fornix and reach the mamillary bodies. In fact, the fornix constitutes a major inflow and output pathway from the hippocampus and medial temporal lobe. It provides a direct source of input from the hippocampal formation to the anterior thalamic nuclei $[8,9]$ and in turn, the anterior thalamic nuclei project to the subicular and postsubicular cortical regions [10]. Furthermore, cholinergic fibers from the basal forebrain, including the septal nuclei and the nucleus basalis of Meynert run through the fornix. 
Lesioning the fornix in experimental animals and also in humans results in major memory deficits [10-12].

In the present study, we addressed three research questions. Does DBS of the fornix in an animal model of memory impairment result in therapeutic benefits in memory functions? Does it result in behavioural side-effects? Which stimulation parameters deliver the most substantial outcome? In order to examine potential memory improvements by DBS, we injected rats with scopolamine, a muscarinic acetylcholine receptor antagonist, to induce memory dysfunction. Justification for this purpose has been provided by the cholinergic hypothesis of geriatric memory impairment [13-17]. The scopolamine model is used extensively for preclinical testing of new substances designed to treat cognitive dysfunctions [13-16]. We evaluated the effects of DBS on memory functions using the object location task (OLT) [18] and potential behavioural side-effects, on anxiety or general motor activity, using the open field (OF) test. The effects of different stimulation parameters, including high and low frequency, as well as high and low current intensities, were examined.

\section{MATERIALS AND METHODS}

\section{Subjects}

All procedures involving animals were carried out with approval of the Animal Experiments and Ethics Committee of Maastricht University. Adult male Sprague Dawley rats (body weight 300-350 $\mathrm{g}$ at the time of surgery) from the Central Animal Facility (Maastricht University, the Netherlands) were individually housed in Makrolon ${ }^{\mathrm{Tm}}$ cages, with rat chow and water available ad libitum. The colony room was maintained at a temperature of $21 \pm 1{ }^{\circ} \mathrm{C}$ and on a reversed 12:12 h light:dark cycle. All experimental manipulations were conducted during the dark phase under red light, when rodents are most active.

\section{Experimental groups}

Rats were randomly assigned to one of the following groups: Sham $(n=11)$ or Fornix DBS $(\mathrm{n}=10)$.

\section{Surgical Procedure}

For the Fornix DBS group, stimulation electrodes were implanted bilaterally in the forniceal region using a rodent stereotact (Stoelting, Wood Dale, IL, USA, model 51653). We aimed not to enter the fornix to prevent damage to the white matter, and placed our electrodes in close vicinity to the fornix. Rats were anesthetized with Isoflurane (IsoFlo ${ }^{\oplus}$, Abbott Laboratories Ltd, Berkshire, Great Britain) throughout the procedure. 
After exposing the skull, the rat received two burrholes at the level of the fornix in order to implant a bilateral electrode construction (coordinates from Bregma according to the Rat Brain atlas of Paxinos and Watson [19]: AP -1.88, ML 1.3, DV -8.2). In addition, five holes were then drilled into the skull and stainless steel screws were placed to provide support in anchoring the electrode with dental acrylic (Paladur, Heraeus Kulzer GmbH, Hanau, Germany). Stimulations were applied bilaterally by using two gold-plated coaxial electrodes with an inner wire of a platinum-iridium combination ( $250 \mu \mathrm{m}$ shaft diameter, $50 \mu \mathrm{m}$ tip diameter; Technomed, Beek, The Netherlands). More details about the electrode construction and surgery have been published before [20, 21]. Sham rats underwent the same surgical procedure with electrode implantation. They were, however, not stimulated.

\section{Drugs}

Scopolamine hydrobromide (Acros Organics BVBA, Geel, Belgium) was dissolved in vehicle (saline; $0.9 \% \mathrm{NaCl}$ ) and administered (i.p.) at a dose of $0.1 \mathrm{mg} / \mathrm{kg}$ (in $1 \mathrm{ml} / \mathrm{kg}$ ) 30 min before the first trial.

\section{Deep brain stimulation}

Rats were tested with the following conditions: (i) with attachment of stimulation cable (off stimulation), and (ii) with DBS at various amplitudes $(50 \mu \mathrm{A}, 100 \mu \mathrm{A}$ and $200 \mu \mathrm{A}$ ), and $100 \mathrm{~Hz}$ or $10 \mathrm{~Hz}$ stimulation frequency. The pulse width was set at $100 \mu$ s. The mode of stimulation was bipolar in all cases (the inner platinum-iridium wire functions as the negative contact and the outer stainless steel electrode as the positive contact). Stimulation was performed in a descending manner ranging from $200 \mu \mathrm{A}, 100 \mu \mathrm{A}$ and $50 \mu \mathrm{A}$ to investigate the effect of a relatively high, medium, and low current density. Taking into account the electrode diameter and exposed surface area, the charge density per phase generated by currents in the $50-300 \mu \mathrm{A}$ approximates that used in humans. It was shown before that $200 \mu \mathrm{A}$ was the most effective parameter for mood improvement $[22,23]$. The frequency applied was low $(10 \mathrm{~Hz})$ and high $(100 \mathrm{~Hz})$ in order to determine whether changes were dependent on the stimulation frequency. Stimuli were delivered using a World Precision Instrument digital stimulator (DS8000, WPI, Berlin, Germany) and stimulus isolators (DLS100, WPI, Berlin, Germany). Stimulation started 2 min before the test and lasted for the duration of each test session, as it was shown that changes in behavior can occur immediately after the onset of stimulation $[5,21$, $23,24]$. Each animal had at least a $24 \mathrm{~h}$ stimulation-off period before the next session started. 


\section{Behavioural Testing}

\section{Object Location Task}

Following the first week of recovery animals were handled daily for one week. Handling involved weighing and procedures of injection. The rats were also allowed to explore the arena of the OLT and its objects in the same week. The apparatus consisted of a circular arena, $83 \mathrm{~cm}$ in diameter, in which half of the $40 \mathrm{~cm}$ high walls was made of grey and the other half of transparent polyvinyl chloride. The arena was placed in such a manner that the light intensity was equal in the different parts of the apparatus (approximately 20 lux). The experimental paradigm was adapted from Rutten and colleagues [18].

Two pre-tests with vehicle injections but without stimulation were carried out before the actual experiment. This was done to habituate the rats to the testing procedures. Objects were presented in a counterbalanced order according to a fully-balanced design, in which each rat obtained a different order of objects to avoid preferences for particular objects or locations.

In the first trial (T1) two objects were placed in a symmetrical position about $10 \mathrm{~cm}$ away from the walls. The different objects were: (A) a standard $1 \mathrm{~L}$ transparent glass bottle (diameter $10 \mathrm{~cm}$, height $22 \mathrm{~cm}$ ) filled with water, (B) plastic cups (diameter 7.8 $\mathrm{cm}$, height $9 \mathrm{~cm}),(\mathrm{C})$ aluminium containers $(7.5 \mathrm{~cm} \times 7.5 \mathrm{~cm} \mathrm{x} 10 \mathrm{~cm})$ and (D) ceramic pyramids $(11.5 \mathrm{~cm} \times 11.5 \mathrm{~cm} \times 9.5 \mathrm{~cm})$. Objects were filled with regular sand cement so that the animals could not move the objects. Rats were given scopolamine injections $30 \mathrm{~min}$ before $\mathrm{T} 1$ in which the animal was allowed to explore the two identical objects for $3 \mathrm{~min}$. A rat was always placed in the apparatus facing the wall at the middle of the transparent segment. Two minutes before placing the rat into the testing arena stimulation started in a separate box and continued while the rats were in the arena. Sham rats underwent the same procedures but without being stimulated. After T1 the rat was put back in its home cage and the second trial (T2) followed after $90 \mathrm{~min}$. In $\mathrm{T} 2$ one of the objects presented before was moved $10 \mathrm{~cm}$ to the front or back of the arena. Again the rats were stimulated 2 min before as well as during T1. Six different stimulation parameters in the following order were used in 6 consecutive sessions: 100 $\mathrm{Hz}+200 \mu \mathrm{A}, 10 \mathrm{~Hz}+200 \mu \mathrm{A}, 100 \mathrm{~Hz}+100 \mu \mathrm{A}, 10 \mathrm{~Hz}+100 \mu \mathrm{A}, 100 \mathrm{~Hz}+50 \mu \mathrm{A}$ and $10 \mathrm{~Hz}+50 \mu \mathrm{A}$. Each animal had at least a $24 \mathrm{~h}$ stimulation-off period before the next session started.

The times spent in exploring each object in T1 and T2 were recorded manually using a personal computer. Exploration was defined as follows: directing the nose to the object at a distance of no more than $2 \mathrm{~cm}$ or touching the object with the nose. Sitting on top of the object or biting it was not considered as exploratory behaviour. In order to avoid the presence of olfactory cues the objects were cleaned with an ethanol solution before each trial. 
Total exploration time during T1 and T2 (e1 and e2, respectively) was considered as the sum of time spent at both objects. Discrimination performance $(\mathrm{d} 2)$ was calculated as follows (time at object at novel position - time at objects in old position)/e2. Side bias was determined by the time at the left or right object, independent of the novel or old position of the object.

\section{Open Field}

The OF consisted of a clear Plexiglas square arena measuring $100 \times 100 \mathrm{~cm}$ with $40 \mathrm{~cm}$ high walls and a dark floor as described in Lim et al. [25]. The test measures time spent and number of entries into the inner and outer zone. More time spent in the centre, indicates increased exploration, and is defined as reduced anxiety. The distance travelled provides an indication of the rat's locomotor activity. Animals were individually placed in the centre of the OF arena and were allowed to move freely in the arena for $5 \mathrm{~min}$. DBS with the most optimal stimulation parameters derived from the OLT (100 Hz and $100 \mu \mathrm{A}$ ) was performed 2 min before testing as well as $5 \mathrm{~min}$ throughout the OF session. Sham animals were attached to cables, but not stimulated. The behavior of each rat was recorded on a computer using Ethovision tracking software (Ethovision, Noldus Information Technology, Wageningen, The Netherlands). The software automatically calculated and analyzed data including the locomotion/distance moved and time spent in the different areas of the open field. The inner zone of $70 \times 70 \mathrm{~cm}$ was demarcated manually on the computer, leaving a surrounding outer zone of $\mathrm{ca} .15 \mathrm{~cm}$ width. After each trial the OF was cleaned with an ethanol solution to diminish odours of other rats.

\section{Histological analysis}

At the end of the experiments an overdose of pentobarbital (Apotheek Faculteit Diergeneeskunde, Utrecht, The Netherlands) was given and rats underwent perfusion-fixation first with Tyrode solution ( $\mathrm{NaCl}, \mathrm{KCl}, \mathrm{CaCl}_{2}, \mathrm{MgCl}_{2}, \mathrm{NaH}_{2} \mathrm{PO}_{4}, \mathrm{NaHCO}_{3}$, Glucose, distilled water) followed by Somogyi solution (4\% Paraformaldehyde, Picric Acid, PBS, Glutaraldehyde, distilled water). To prevent the development of postperfusion artifacts, brains were fixed in fresh fixative (same content as in the Somogyi solution but lacking Glutaraldehyde) at $4{ }^{\circ} \mathrm{C}$. After 2 hours, the brains were gently removed and stored in $1 \% \mathrm{NaN}_{3}$ in $4{ }^{\circ} \mathrm{C}$.

Brains were embedded in 10\% gelatin from porcine skin (Sigma-Aldrich, Zwyndrecht, The Netherlands), and cut into $30 \mu \mathrm{m}$ slices in the frontal plane using a vibratome (Leica ${ }^{\oplus}$, Wetzlar, Germany). Slices were immediately transferred into $1 \% \mathrm{NaN}_{3}$. We collected the sections containing the electrode trajectories, mounted on gelatine coated slides and air dried overnight. Subsequently, we performed a standard hematoxylineosin staining. After dehydration, sections were coverslipped with Pertex mounting medium (Histolab Products AB, Gothenburg, Sweden) and photographed under bright field microscopy (Olympus ${ }^{\circledast}$ ). 


\section{Statistical analysis}

For the OLT, e1 and e2 for the vehicle (no stimulation) were analyzed by a paired sample t-test in order to assess a priori differences between the two groups. For the scopolamine sessions a mixed three-way ANOVA with Current and Frequency as within-subjects factor and Group (sham and DBS fornix) as a between-subjects factor was used. In order to test whether the discrimination performance, $\mathrm{d} 2$, deviated from zero, a one-sample t-test was performed for each observation. Side bias in the second trial was evaluated within groups using a paired samples t-tests. For the OF test, the data were analyzed using an independent samples t-test. All p-values $<0.05$ were considered significant.

\section{RESULTS}

\section{Histological evaluation of electrode tips}

There were 10 rats in the DBS fornix group in total, so we verified 20 electrode tips. We lost no animals. The bilateral electrodes were all implanted in the vicinity of the fornix (Figs. 1A and 1B). The electrodes were situated ventral, medial or lateral to the
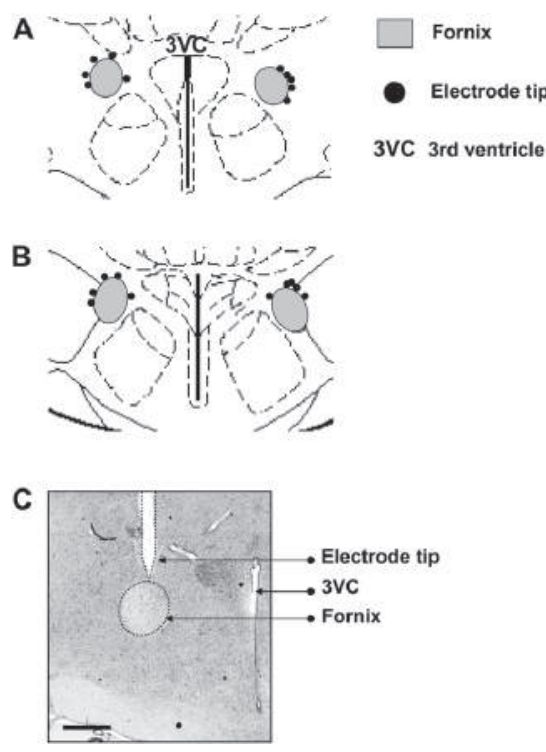

Figure 1: Histological evaluation of electrode tips. Anatomical locations of bilateral stimulation sites close to the fornix at (A) bregma level $1.60 \mathrm{~mm}$ (24) and (B) $1.80 \mathrm{~mm}$ (24). Sites were localized by microscopic examination of histologically prepared tissue. C) Illustrative coronal section showing the histological verification of the electrode location in the vicinity of the fornix (bar $=400 \mu \mathrm{m}$ ). 
fornix. We found no differences between the ventrally, medially or laterally placed electrodes in terms of behavioural outcome. With the current stimulation setting, we found no evidence for histological damage observable with a hematoxylin-eosin staining (Fig. 1C).

\section{Object Location Task}

There were no significant a priori differences between sham and DBS fornix rats in the exploration levels e 1 and e 2 of the first and second trial in the vehicle and off stimulation condition. Only one rat in the fornix DBS group had to be excluded due

Table 1: Mean exploration times (in s) of rats with sham surgery and DBS on the objects in T1 (e1) and T2 (e2). In T2 the time the animals spent on the right and left side was recorded to examine possible side bias. Both groups received vehicle and scopolamine injections as indicated, but only the rats with fornix DBS were stimulated. Entries represent mean (SEM). Bold values characterize significant effects.

\begin{tabular}{|c|c|c|c|c|c|c|}
\hline & & Sham & Fornix & & e2 & Side Bias (left vs right) \\
\hline \multirow[t]{4}{*}{ Vehicle } & e1 & $43.6(2.9)$ & \multirow[t]{4}{*}{ Off stim } & $42.6(2.9)$ & \multirow{4}{*}{$\begin{array}{l}\underline{e 1}: \mathrm{t}(19)=0.23, \text { n.s. } \\
\underline{e 2}: \mathrm{t}(19)=0.81, \text { n.s. }\end{array}$} & sham: \\
\hline & e2 & $42.9(2.7)$ & & $46.4(3.6)$ & & $\mathrm{t}(10)=0.52$, n.s. \\
\hline & time left & $22.3(2.5)$ & & $23.7(3.4)$ & & DBS fornix: \\
\hline & time right & $20.5(2.0)$ & & $22.8(2.5)$ & & $\mathrm{t}(9)=0.19$, n.s. \\
\hline \multirow{4}{*}{$\begin{array}{l}\text { Scopolamine } \\
\text { session } 1\end{array}$} & e1 & $44.2(3.3)$ & \multirow{4}{*}{$\begin{array}{l}100 \mathrm{~Hz} \\
200 \mu \mathrm{A}\end{array}$} & $38.0(5.5)$ & \multirow{4}{*}{$\begin{array}{l}\frac{e 1}{\text { curr: }} \\
\mathrm{F}(2 ; 36)=0.051, \text { n.s. } \\
\text { curr }^{*} \text { group: }\end{array}$} & sham: \\
\hline & e2 & $42.8(3.6)$ & & $38.4(3.5)$ & & $\mathrm{t}(10)=1.25$, n.s. \\
\hline & time left & $16.1(1.8)$ & & $18.3(3.6)$ & & DBS fornix: \\
\hline & time right & $14.5(2.0)$ & & $12.8(1.7)$ & & $\mathrm{t}(9)=1.22$, n.s. \\
\hline \multirow{4}{*}{$\begin{array}{l}\text { Scopolamine } \\
\text { session } 2\end{array}$} & e1 & $40.3(3.5)$ & \multirow{4}{*}{$\begin{array}{l}10 \mathrm{~Hz} \\
200 \mu \mathrm{A}\end{array}$} & $33.7(4.2)$ & \multirow{4}{*}{$\begin{array}{l}\mathrm{F}(2 ; 36)=0.512, \text { n.s. } \\
\text { freq: } \\
\mathrm{F}(1 ; 18)=1.42, \text { n.s. } \\
\text { freq }{ }^{*} \text { group: } \\
\mathrm{F}(1 ; 18)=0.002 \text {, n.s. }\end{array}$} & sham: \\
\hline & e2 & $34.9(4.2)$ & & $40.2(4.1)$ & & $\mathrm{t}(10)=0.05$, n.s. \\
\hline & time left & $17.4(2.3)$ & & $17.3(3.3)$ & & DBS fornix: \\
\hline & time right & $17.5(2.4)$ & & $22.9(1.9)$ & & $\mathrm{t}(9)=1.64$, n.s. \\
\hline \multirow{4}{*}{$\begin{array}{l}\text { Scopolamine } \\
\text { session } 3\end{array}$} & e1 & $35.5(4.3)$ & \multirow{4}{*}{$\begin{array}{l}100 \mathrm{~Hz} \\
100 \mu \mathrm{A}\end{array}$} & $36.7(5.9)$ & curr ${ }^{*}$ freq: & sham: \\
\hline & e2 & $30.4(2.0)$ & & $43.3(4.4)$ & $\mathrm{F}(2 ; 36)=7.15, \mathrm{p}<0.01$ & $\mathrm{t}(10)=0.76$, n.s. \\
\hline & time left & $15.9(1.3)$ & & $25.2(3.4)$ & curr ${ }^{*}$ freq ${ }^{*}$ group: & DBS fornix: \\
\hline & time right & $14.4(1.5)$ & & $18.1(3.3)$ & $\mathrm{F}(2 ; 36)=0.91$, n.s. & $\mathrm{t}(9)=1.37$, n.s. \\
\hline \multirow{4}{*}{$\begin{array}{l}\text { Scopolamine } \\
\text { session } 4\end{array}$} & e1 & $28.5(2.7)$ & \multirow{4}{*}{$\begin{array}{l}10 \mathrm{~Hz} \\
100 \mu \mathrm{A}\end{array}$} & $31.6(3.5)$ & & sham: \\
\hline & e2 & $24.1(3.4)$ & & $28.9(2.8)$ & $\underline{\text { e } 2 \text { urr: }}$ & $\mathrm{t}(10)=0.43$, n.s. \\
\hline & time left & $11.7(1.6)$ & & $15.2(1.7)$ & $F(2 ; 36)=0.81$, n.s. & DBS fornix: \\
\hline & time right & $12.4(2.2)$ & & $13.8(2.1)$ & curr ${ }^{*}$ group: & $\mathrm{t}(9)=0.53$, n.s. \\
\hline \multirow{4}{*}{$\begin{array}{l}\text { Scopolamine } \\
\text { session } 5\end{array}$} & e1 & $37.3(2.9)$ & \multirow{4}{*}{$\begin{array}{l}100 \mathrm{~Hz} \\
50 \mu \mathrm{A}\end{array}$} & $36.4(3.8)$ & $\mathrm{F}(2 ; 36)=2.42$, n.s. & sham: \\
\hline & e2 & $35.1(3.9)$ & & $35.7(3.6)$ & & $\mathrm{t}(10)=1.7$, n.s. \\
\hline & time left & $15.8(1.4)$ & & $16.2(2.0)$ & $\begin{array}{l}\mathrm{F}(1 ; 18)=2.36, \text { n.s. } \\
\text { frea * oroup. }\end{array}$ & DBS fornix: \\
\hline & time right & $19.2(2.7)$ & & $19.6(2.8)$ & $\mathrm{F}(1 ; 18)=0.04$, n.s. & $\mathrm{t}(9)=1.02$, n.s. \\
\hline \multirow{4}{*}{$\begin{array}{l}\text { Scopolamine } \\
\text { session } 6\end{array}$} & e1 & $29.9(3.0)$ & \multirow{4}{*}{$\begin{array}{l}10 \mathrm{~Hz} \\
50 \mu \mathrm{A}\end{array}$} & $32.5(2.4)$ & curr ${ }^{*}$ freq: & sham: \\
\hline & e2 & $28.3(3.4)$ & & $27.9(4.0)$ & $\mathrm{F}(2 ; 36)=4.59, \mathrm{p}<0.02$ & $\mathrm{t}(10)=3.02, \mathrm{p}<0.02$ \\
\hline & time left & $12.2(1.7)$ & & $11.3(1.8)$ & curr ${ }^{\star}$ freq ${ }^{\star}$ group: & DBS fornix: \\
\hline & time right & $16.1(1.9)$ & & $16.6(2.4)$ & $\mathrm{F}(2 ; 36)=0.54, \mathrm{n} . \mathrm{s}$ & $t(9)=3.45, p<0.01$ \\
\hline
\end{tabular}


to low exploration levels and was not considered in the following analysis. All current, frequency and interactions within groups were not significant for exploration times during subsequent scopolamine and stimulation sessions (all F's $<0.9$, n.s.). Only one interaction between current density and frequency was found in e1. There were no side biases in the second trial in all sessions, except for the last scopolamine session (Table 1).

For the time ratio of discrimination between the displaced and the familiar objects, $\mathrm{d} 2$, we found a significant effect of current $(\mathrm{F}(2 ; 36)=3.61, \mathrm{p}<0.05)$. There was no significant effect of frequencyor the interaction between frequency, current and groups (all F's $<2.05$, n.s.). However, the fornix stimulated group performed significantly better on the memory task than the sham group (Group effect: $\mathrm{F}(1,10)<18.14, \mathrm{p}<0.001$, Fig. 2). Analysis of the individual stimulation conditions revealed that the $10 \mathrm{~Hz}+200$ $\mu \mathrm{A}$ and both $100 \mu \mathrm{A}$ conditions significantly improved the memory performance (all t's $(8)<2.85, \mathrm{p}<0.05)$. In the sham group it was found that only in the fifth scopolamine session the discrimination performance was below zero $(\mathrm{t}(10)=2.96, \mathrm{p}<0.02)$.

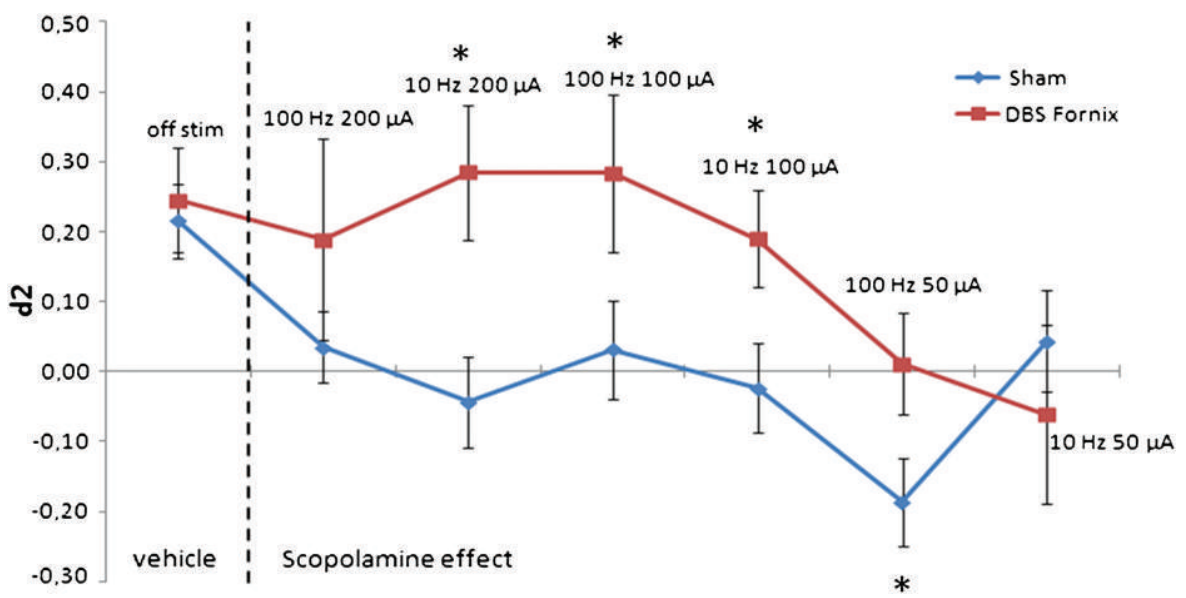

Figure 2: Ratio time spent at the new object for sham and Fornix DBS rats in the OLT. In the first session stimulation was off and saline was injected in all rats. In the following 6 sessions scopolamine was injected and rats in the fornix DBS group were stimulated with the parameters mentioned above. Sham rats only received scopolamine and were not stimulated. There was a current effect in the DBS fornix group, with the most optimal effects at 100 (and $100 \mathrm{~Hz}$ ) and $200 \mu \mathrm{A}$ (and $10 \mathrm{~Hz}$ ). In the sham group the discrimination ratio was significantly below zero at the fifth scopolamine session, indicating a particular bad memory performance on this day. ${ }^{*}$ indicates a p-value $<0.05$, showing a significant difference. 


\section{Open field}

There was no significant difference between fornix DBS and sham rats for the times spent in corners, walls, centre or distance moved in the OF ( $\mathrm{t}(19)=1.99$, n.s., Table 2).

Table 2: Time spent in the different areas of the Open Field and the total distance moved. There was no significant difference between sham operated rats and rats with fornix DBS. Data are presented as mean \pm SEM.

\begin{tabular}{lllll}
\hline & Corners $[\mathbf{s}]$ & Walls $[\mathbf{s}]$ & Centre $[\mathbf{s}]$ & Distance moved $[\mathrm{cm}]$ \\
\hline Sham & $123 \pm 6$ & $132 \pm 4$ & $44 \pm 3$ & $3653 \pm 245$ \\
DBS Fornix & $104 \pm 7$ & $140 \pm 5$ & $54 \pm 8$ & $3329 \pm 279$ \\
\hline
\end{tabular}

\section{DISCUSSION}

We found that DBS of the forniceal region improved impaired spatial memory performance, which was induced by injections of scopolamine in order to produce a rat model of experimental dementia. This memory improvement was substantial and became comparable to the performance of controls. In the OF we found no side-effects on locomotor and anxiety-related behaviours. Stimulation of parts of the limbic system is known to induce strong effects on anxiety levels since it is interconnected with regions such as the periaqueductal gray, amygdala, hypothalamus and the nucleus accumbens. Stimulation of the first region induces fear and anxiety behaviour in animals [26] while stimulation of the latter produces anxiolytic effects in humans [1].

Interestingly, for the time ratio of discrimination between the displaced and the familiar objects, $\mathrm{d} 2$, we found a significant current density threshold rather than a frequency effect, since both $10 \mathrm{~Hz}$ and $100 \mathrm{~Hz}$ were efficient. However, in the case of $10 \mathrm{~Hz}$, we needed higher currents to reach therapeutic effects than when $100 \mathrm{~Hz}$ was applied. These findings suggest a total energy/current density threshold rather than a frequency effect. The latter is well-known for DBS of the subthalamic nucleus in patients with Parkinson's disease as well as corresponding animal models, where only higher frequencies produce therapeutic effects. The major difference is that in Parkinson's disease and in most of the other neurological disorders grey matter (cell bodies, dendrites, and small axons) is usually stimulated to cause an overall inhibition [27, 28], although some excitation also occurs. Furthermore, in some DBS-implanted epilepsy patients as well as in animal models high frequency in combination with high current stimulation of the anterior thalamic nucleus, impairs, rather than facilitates cognitive function due to an overall inhibition of the limbic structures $[22,29]$. Note that all structures mentioned above, consist of grey matter and high frequencies are used to cause an overall 
inhibition. In our study, we aimed to stimulate white matter (large myelinated axons) and found memory enhancement. Our hypothesis is that this effect is accomplished by driving the fornix activity, both orthodromically as well as antidromically. This is supported by the view that large myelinated axons produce excitatory responses upon electrical stimulation [30].

$\mathrm{AD}$ is a prototypical neurodegenerative disease and the most common form of dementia. It is characterized by a series of abnormalities in the neocortex, the entorhinal area, hippocampus, amygdala, nucleus basalis of Meynert, anterior thalamus and several brain stem monoaminergic nuclei [31]. The distribution and spread of neural abnormalities in AD follow characteristic patterns that are area-specific and even cellspecific. Besides degeneration of glutamatergic pyramidal neurons and interneurons, cholinergic neurons in the nucleus basalis of Meynert, the medial septal nucleus, and the diagonal band of Broca which provide the principal cholinergic pathways to the neocortex and hippocampus, are also destroyed [31, 32]. These lesions have profound clinical consequences such as memory impairments. Abnormalities of the entorhinal cortex, hippocampus and in the association areas of the neocortex, which are believed to be related to alterations in the basal forebrain cholinergic systems, contribute to the memory difficulties and attention deficits of this disease [31, 32]. In our study, we applied a cholinergic amnesia model and injected scopolamine to induce memory impairment. It has been shown that cholinergic activity in the hippocampus is associated with memory and systemic administration of scopolamine impairs hippocampal-dependent learning and memory in rats [33-35]. We are aware that the model applied in our study does not resemble human $\mathrm{AD}$, for instance it lacks $\mathrm{AD}$ specific neuropathology and the progressive nature of the disease. Nevertheless, it is an adequate and widely used model to evaluate the potency of experimental therapies on cognitive functions.

As mentioned before, cholinergic fibers from the basal forebrain and from the septohippocampal system run through the fornix, which in turn establishes a connection between the hippocampus and the anterior thalamic nucleus. The hippocampus itself contains muscarinic acetylcholine receptors and it is probable, that the blockade of these receptors through scopolamine administration leads to impaired memory performance in the OLT [33]. Since DBS of the fornix is thought to drive its output, it reinforces the connection between the hippocampus and the anterior thalamic nucleus and thus counteracts the effects of scopolamine receptor blockade in the hippocampus. A functional interaction between hippocampus and anterior thalamic nucleus is necessary for spatial memory and conditional learning as seen in the study of Dumont et al. [36].

To date only two studies have been published, which investigated DBS within the fornix vicinity in patients suffering from $\mathrm{AD}$ and morbid obesity $[5,6]$. Using imaging techniques, both authors have found that stimulation activated medial temporal lobe 
structures like the hippocampus and the parahippocampal gyrus and thus improved hippocampus-dependent memory performance. This is in line with our hypothesis that stimulation of the fornix drives its output and triggers the activity of the hippocampus and/or the anterior nucleus of the thalamus. One way of action of DBS might be increasing neurotransmitter release and local or transsynaptic modulation of neural activity at the cellular level [37] as well as restore basal activity levels within dysregulated brain regions at circuit levels $[6,38]$. Stimulation of the entorhinal cortex, for instance, has been shown to induce hippocampal neurogenesis $[39,40]$ and to improve performance in cognitive tasks [41]. Our hypothesis requires more investigation and further studies are needed to clarify inhibitory as well as excitatory responses to DBS and their implications in memory enhancement.

Our findings identify the fornix as a key region in controlling spatial memory functions. DBS of this region, using tailored stimulation parameters, has the potential to improve memory functions in conditions characterised by memory impairment.

\section{ACKNOWLEDGEMENTS}

This study was funded by the Internationale Stichting Alzheimer Onderzoek (ISAO). 


\section{REFERENCES}

1. Bewernick BH, Hurlemann R, Matusch A, Kayser S, Grubert C, Hadrysiewicz B, et al. Nucleus Accumbens Deep Brain Stimulation Decreases Ratings of Depression and Anxiety in TreatmentResistant Depression. Biological Psychiatry. 2010;67(2):110-6.

2. Lozano AM, Mayberg HS, Giacobbe P, Hamani C, Craddock RC, Kennedy SH. Subcallosal Cingulate Gyrus Deep Brain Stimulation for Treatment-Resistant Depression. Biological Psychiatry. 2008;64(6):461-7.

3. Malone Jr DA, Dougherty DD, Rezai AR, Carpenter LL, Friehs GM, Eskandar EN, et al. Deep Brain Stimulation of the Ventral Capsule/Ventral Striatum for Treatment-Resistant Depression. Biological Psychiatry. 2009;65(4):267-75.

4. Denys D, Mantione M, Figee M, van den Munckhof P, Koerselman F, Westenberg H, et al. Deep Brain Stimulation of the Nucleus Accumbens for Treatment-Refractory Obsessive-Compulsive Disorder. Arch Gen Psychiatry. 2010;67(10):1061-8.

5. Hamani C, McAndrews MP, Cohn M, Oh M, Zumsteg D, Shapiro CM, et al. Memory enhancement induced by hypothalamic/fornix deep brain stimulation. Annals of Neurology. 2008;63(1):119-23.

6. Laxton AW, Tang-Wai DF, McAndrews MP, Zumsteg D, Wennberg R, Keren R, et al. A phase I trial of deep brain stimulation of memory circuits in Alzheimer's disease. Annals of Neurology. 2010;68(4):521-34.

7. Rajmohan V ME. The limbic system. Indian J Psychiatry. 2007;49:132-9.

8. Aggleton JP DR, Mishkin M. The origin, course, and termination of the hippocampothalamic projections in the macaque. J Comp Neurol. 1986;243(3):409-21.

9. Neave N, Lloyd S, Sahgal A, Aggleton JP. Lack of effect of lesions in the anterior cingulate cortex and retrosplenial cortex on certain tests of spatial memory in the rat. Behavioural Brain Research. 1994;65(1):89-101.

10. van Groen T WJM. Connection of the retrosplenial granular a cortex in the rat. J Comp Neurol. 1990;300(4):593-606.

11. Tsivilis D, Vann SD, Denby C, Roberts N, Mayes AR, Montaldi D, et al. A disproportionate role for the fornix and mammillary bodies in recall versus recognition memory. Nat Neurosci. 2008;11(7):83442.

12. Browning PGF GD, Croxson PL, and Baxter MG. Severe Scene Learning Impairment, but Intact Recognition Memory, after Cholinergic Depletion of Inferotemporal Cortex Followed by Fornix Transection. Cereb Cortex. 2010;20(2):282-93.

13. Barak S, Weiner I. Towards an animal model of an antipsychotic drug-resistant cognitive impairment in schizophrenia: scopolamine induces abnormally persistent latent inhibition, which can be reversed by cognitive enhancers but not by antipsychotic drugs. The International Journal of Neuropsychopharmacology. 2009;12(02):227-41

14. Buccafusco J, Terry A, Webster S, Martin D, Hohnadel E, Bouchard K, et al. The scopolamine-reversal paradigm in rats and monkeys: the importance of computer-assisted operant-conditioning memory tasks for screening drug candidates. Psychopharmacology. 2008;199(3):481-94.

15. Loiseau F, Dekeyne A, Millan M. Pro-cognitive effects of 5-HT receptor antagonists in the social recognition procedure in rats: implication of the frontal cortex. Psychopharmacology. 2008;196(1):93104.

16. Vaisman N, Pelled D. n- phosphatidylserine attenuated scopolamine-induced amnesia in middleaged rats. Progress in Neuro-Psychopharmacology and Biological Psychiatry. 2009;33(6):952-9. 
17. Klinkenberg I, Blokland A. The validity of scopolamine as a pharmacological model for cognitive impairment: A review of animal behavioral studies. Neuroscience \&amp; Biobehavioral Reviews. 2010;34(8):1307-50.

18. Rutten K, Van Donkelaar EL, Ferrington L, Blokland A, Bollen E, Steinbusch HWM, et al. Phosphodiesterase Inhibitors Enhance Object Memory Independent of Cerebral Blood Flow and Glucose Utilization in Rats. Neuropsychopharmacology. 2009;34(8):1914-25.

19. Paxinos G, Watson C. The rat brain in steroetaxic coordinates. 1998.

20. Tan SKH, Vlamings R, Lim LW, Sesia T, Janssen MLF, Steinbusch HWM, et al. Experimental Deep Brain Stimulation in Animal Models. Neurosurgery. 2010;67(4):1073-80

21. Temel Y, Boothman LJ, Blokland A, Magill PJ, Steinbusch HWM, Visser-Vandewalle V, et al. Inhibition of 5-HT neuron activity and induction of depressive-like behavior by high-frequency stimulation of the subthalamic nucleus. Proceedings of the National Academy of Sciences. 2007;104(43):17087-92.

22. Hamani C, Diwan M, Isabella S, Lozano AM, Nobrega JN. Effects of different stimulation parameters on the antidepressant-like response of medial prefrontal cortex deep brain stimulation in rats. Journal of Psychiatric Research. 2010;44(11):683-7.

23. Hamani C, Mayberg H, Stone S, Laxton A, Haber S, Lozano AM. The Subcallosal Cingulate Gyrus in the Context of Major Depression. Biological Psychiatry. 2011;69(4):301-8.

24. Sesia T, Temel Y, Lim LW, Blokland A, Steinbusch HWM, Visser-Vandewalle V. Deep brain stimulation of the nucleus accumbens core and shell: Opposite effects on impulsive action. Experimental neurology. 2008;214(1):135-9.

25. Lim LW TY, Visser-Vandewalle V, Steinbusch H, Schruers K, Hameleers R, Esquivel G, Griez E, Blokland A. Effect of buspirone on the behavioral regulation of rats in low versus high anxiety conditions. Arzneimittelforschung. 2008;58(6):269-76.

26. Lim LW, Blokland A, Tan S, Vlamings R, Sesia T, Aziz-Mohammadi M, et al. Attenuation of fear-like response by escitalopram treatment after electrical stimulation of the midbrain dorsolateral periaqueductal gray. Experimental neurology. 2010;226(2):293-300. eng.

27. Temel Y, Cao C, Vlamings R, Blokland A, Ozen H, Steinbusch HWM, et al. Motor and cognitive improvement by deep brain stimulation in a transgenic rat model of Huntington's disease. Neuroscience letters. 2006;406(1-2):138-41.

28. Vlamings R, Visser-Vandewalle V, Kozan R, Kaplan S, Steinbusch HWM, Temel Y. Bilateral high frequency stimulation of the subthalamic nucleus normalizes COX activity in the substantia nigra of Parkinsonian rats. Brain Research. 2009;1288(0):143-8.

29. Fisher R, Salanova V, Witt T, Worth R, Henry T, Gross R, et al. Electrical stimulation of the anterior nucleus of thalamus for treatment of refractory epilepsy. Epilepsia. 2010;51(5):899-908.

30. Ranck JB. Which elements are excited in electrical stimulation of mammalian central nervous system: A review. Brain Res. 1975;98(3):417-40.

31. Schliebs R, Arendt T. The cholinergic system in aging and neuronal degeneration. Behavioural Brain Research. 2011;221(2):555-63.

32. Petersen RC, Jack CR. Imaging and Biomarkers in Early Alzheimer's Disease and Mild Cognitive Impairment. Clin Pharmacol Ther. 2009;86(4):438-41.

33. Blokland A, Honig W, Raaijmakers WG. Effects of intra-hippocampal scopolamine injections in a repeated spatial acquisition task in the rat. Psychopharmacology (Berl). 1992;109(3):373-6.

34. Knox LT, Jing Y, Fleete MS, Collie ND, Zhang H, Liu P. Scopolamine impairs behavioural function and arginine metabolism in the rat dentate gyrus. Neuropharmacology. 2011;61(8):1452-62.

35. Hasselmo ME. The role of Acetylcholine in Learning and Memory. Curr Opin Neurobiol. 2009;16(6):710-5. 
36. Dumont JR, Petrides M, Sziklas V. Fornix and retrosplenial contribution to a hippocampo-thalamic circuit underlying conditional learning. Behavioural Brain Research. 2010;209(1):13-20.

37. Kringelbach ML, Green AL, Owen SLF, Schweder PM, Aziz TZ. Sing the mind electric - principles of deep brain stimulation. European Journal of Neuroscience. 2010;32(7):1070-9.

38. Mayberg HS LA, Voon V, McNeely HE, Seminowicz D, Hamani C. Deep brain stimulation for treatment-resistant depression. Neuron. 2005;45:651-60.

39. Toda H, Hamani C, Fawcett AP, Hutchison WD, Lozano AM. The regulation of adult rodent hippocampal neurogenesis by deep brain stimulation. Journal of Neurosurgery. 2008;108(1):132-8.

40. Stone SSD, Teixeira CM, DeVito LM, Zaslavsky K, Josselyn SA, Lozano AM, et al. Stimulation of Entorhinal Cortex Promotes Adult Neurogenesis and Facilitates Spatial Memory. The Journal of Neuroscience. 2011;31(38):13469-84.

41. Shirvalkar P, Seth M, Schiff ND, Herrera DG. Cognitive enhancement with central thalamic electrical stimulation. Proceedings of the National Academy of Sciences. 2006;103(45):17007-12. 




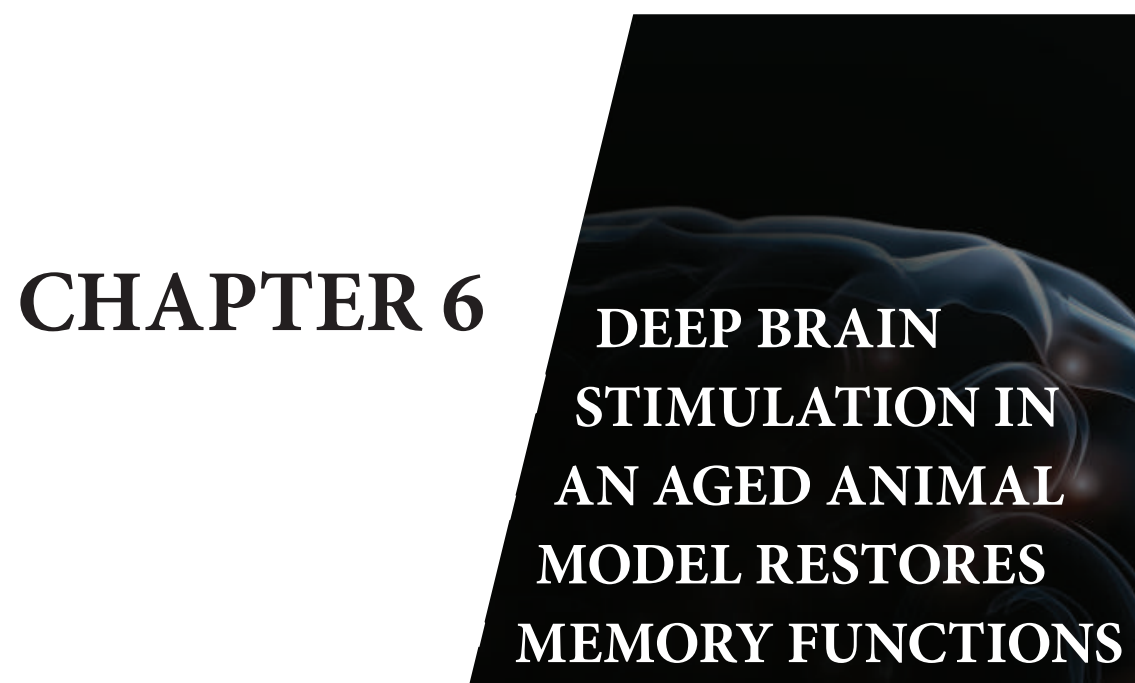

Sarah Hescham, Yasin Temel, Arjan Blokland, LeeWei Lim 


\section{ABSTRACT}

Background: Age is the biggest risk factor for developing dementia and with the anticipated increase in life expectancy, dementia is becoming one of the greatest medical challenge. To date, there is no known cure that can halt or delay the progression of memory loss. Researchers are currently exploring the applicability of deep brain stimulation (DBS) as potential treatment.

Methods: We have used an aged animal model and implanted bilateral DBS electrodes in the fornix and entorhinal cortex. Rats have performed several behavioral tasks: elevated plus maze, light-dark box, open field, water maze and object recognition task. In both memory tasks, we investigated effects on recent as well as remote memory.

Results: We found no indication for anxiety-related behavior following DBS. Fornix DBS rats showed an increased memory performance when we assessed recent memory in a spatial memory task, the water maze. Contrary to this, entorhinal cortex DBS rats showed beneficial effects for remote memory in the object recognition task.

Conclusion: DBS of the fornix and entorhinal cortex has an impact on different memory systems. Yet it seems that fornix DBS is more relevant to improve symptoms of age-dependent dementia. 


\section{INTRODUCTION}

Ageing constitutes one of the major health challenges faced by modern societies. People of old age suffer from cognitive and functional decline. In this respect, the prevalence of dementia also increases with age, from $10 \%$ at 65 years to about $50 \%$ over age 85 [1]. The World Health Organization estimates that 35.6 million people suffer from dementia, a number that is anticipated to triple by 2050 (World Health Organization, 2012). Demented behavior can include wandering, problems with communicating and reasoning, aggression, depression and changes in personality. Alzheimer's disease and vascular dementia are the most common types of dementia, but there are many other conditions that can cause symptoms of dementia. Currently, there have only been advances for symptomatic treatments, which were mostly palliative in nature $[2,3]$, while cures or treatments that delay or reverse memory dysfunctions are unknown. These pharmacological treatments may produce severe side effects and only work for a limited number of patients. Moreover, their effect usually wears off over time [4].

Therefore, researchers have been exploring the application of non-drug based therapies as alternative treatments to alleviate symptoms of dementia-related disorders, such as deep brain stimulation (DBS). The basic principle of this treatment includes electrical stimulation via implanted electrodes in the desired regions of the brain. These stimulation electrodes are connected to an internal pulse generator through insulated wires $[5,6]$. At stimulation settings commonly used in clinical practice, DBS decreases spontaneous firing of neuronal populations and drives axonal projections near the electrodes. Thus, DBS can modulate local and distant targets.

So far only a few studies have investigated the effects of DBS on memory and favorable outcomes were observed following fornix [7-11], entorhinal cortex [12, 13] and nucleus basalis of Meynert DBS [14-16]. However, up to now most studies were conducted in humans, although the use of animal models has played a substantial role in the development and refinement of DBS as a therapy $[6,17,18]$. Potential side effects as well as mechanisms of action can be explored in animals. Currently, there have been a few preclinical studies investigating memory effects following DBS. In an experimental model of dementia, for instance, fornix DBS was able to reverse scopolamine-induced memory deficits in the object location task when certain stimulation parameters were used [9]. Another study, applied entorhinal cortex DBS in mice (1 hour at $130 \mathrm{~Hz}, 90 \mu \mathrm{s}$ pulse width and $50 \mu \mathrm{A}$ ) and also found improvements in spatial memory performance reflected by enhanced water maze scores. Six and a half weeks after surgery, behavioral improvement was accompanied by an observed increase in proliferation in the dentate gyrus, a hippocampal structure maintaining strong connections with the entorhinal cortex [12]. 
Limitations of preclinical studies up to now, however, include the use of young, healthy animals. In the present study we therefore focused on an aged animal model in which the natural aging process leads to cognitive decline. When considering the life span of a laboratory rat, which approximates a maximum of 2.5- 3 years, our aged animals of 2 years seem to be comparable to 60 year old humans [19]. These aged rats may yield more representative data and thus be more translatable to humans. We have implanted DBS electrodes in the fornix and entorhinal cortex and evaluated memory performance, but also anxiety-related side effects in a behavioral task battery. The fornix and entorhinal cortex have been chosen based on the aforementioned studies and in order to stimulate the output and input of the hippocampus, respectively.

\section{METHODS}

\section{Subjects}

Male Sprague Dawley rats 23 months of age were used, their weight ranging between $500-650 \mathrm{~g}$ at time of surgery. The temperature of the colony room was maintained at a temperature of $25 \pm 1{ }^{\circ} \mathrm{C}$ and rats were individually housed in standard cages with rat chow and water available ad libitum. Furthermore, the rats were exposed to a reversed 12:12 $\mathrm{h}$ light dark cycle. All experimental procedures were approved by the Institutional of Animals Care and Use Committee of Nanyang Technological University, Singapore (ARF-SBS/NIE-A 0169 AZ).

\section{Experimental groups}

Rats were randomly assigned to one of the following experimental groups: Sham $(n=12)$, Fornix DBS $(n=11)$, and entorhinal cortex DBS $(n=7)$. The exact timeline of the experimental procedures can be seen in Figure 1.

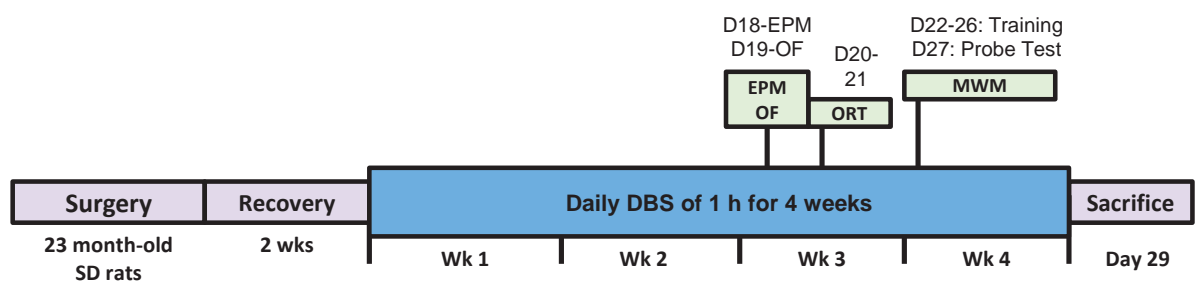

Figure 1: Timeline of the experimental procedures. Please note, DBS was always applied $1 \mathrm{~h}$ before behavioural testing took place. Sham animals were only attached to cables and not stimulated. 


\section{Surgical procedure}

DBS electrodes were implanted bilaterally in the target areas making use of a rodent stereotactic apparatus (Vernier Stereotaxic Instrument, Leica Biosystems, Nussloch, Germany) and Isoflurane of 5\% concentration was used as induction anesthesia and $2.5 \%$ concentration as continuous maintenance. After exposure of the rat skull, two burr holes were made either at the level of the fornix (coordinates from Bregma according to Rat Brain Atlas of Paxinos and Watson [20]: AP: $-1.92 \mathrm{~mm}$; ML: $1.5 \mathrm{~mm}$; DV: $-8.4 \mathrm{~mm}$ ), or entorhinal cortex (AP:-6.84 mm; ML: $4 \mathrm{~mm}$; DV: $-8.6 \mathrm{~mm}$ ). This construct was anchored on the rat skull through stainless steel screws and dental acrylic (Paladur, Heraeus Kulzer GmbH, Hanau, Germany). More detailed information about electrodes and surgical procedures is given in previous publications from our group [21, 22]. Sham rats underwent the same surgical procedure with electrode implantation.

\section{Deep brain stimulation}

Stimulation was performed using a World Precision Instrument digital stimulator (DS8000, WPI, Berlin, Germany) and two stimulus isolators (DLS100, WPI, Berlin, Germany). Rats were attached to cables and the stimulator was switched on 1 hour per day before testing. In the sham group the cable was also connected but this group was not stimulated. Based on previous studies, fornix and entorhinal cortex DBS rats were stimulated with $100 \mathrm{~Hz}, 100 \mu \mathrm{A}$ and $100 \mu$ s pulse width $[9,12]$.

\section{Behavioral testing}

\section{Elevated Plus Maze}

The elevated plus-maze apparatus consisted of two opposing open arms $(50 \mathrm{x} 10 \mathrm{~cm})$ with a $10 \mathrm{~cm}$ high surrounding wall and two opposing enclosed arms $(50 \times 10 \mathrm{~cm})$ with a $50 \mathrm{~cm}$ high surrounding wall. The arms extended from a central platform (10 $\mathrm{x} 10 \mathrm{~cm}$ ). The floor of the open arms, the central platform, and the closed arms were made of black Plexiglass, while the surrounding walls of the arms were made of clear Plexiglass. The maze was elevated $50 \mathrm{~cm}$ from the floor. A rat was always placed on the central platform facing one of the open arms. The cumulative time spent in the open and closed arms was measured for a 5 min period using Noldus Ethovision. The maze was thoroughly cleaned with $70 \%$ ethanol after each session.

\section{Open Field}

The Open Field (OF) consisted of a square arena measuring 40 x $40 \mathrm{~cm}$ with $40 \mathrm{~cm}$ high walls. The center of $20 \times 20 \mathrm{~cm}$ was demarcated manually on the computer, leaving a surrounding outer zone of $20 \mathrm{~cm}$ width. The behavior of each rat was recorded under 
low light condition using a computer with Ethovision tracking software (Ethovision, Noldus Information Technology, Wageningen, The Netherlands).

\section{Morris Water Maze}

The Morris water maze consisted of a black circular pool, which was $128 \mathrm{~cm}$ in diameter and $60 \mathrm{~cm}$ deep and was filled with $30 \mathrm{~cm}$ water. The pool was divided into four quadrants and a $10 \mathrm{~cm}$ circular escape platform was submerged $25 \mathrm{~cm}$ from the wall in the target quadrant of the pool. The position of the platform was kept constant for the duration of the 5 day acquisition period. Water temperature was maintained between $23^{\circ} \mathrm{C}$ and $25^{\circ} \mathrm{C}$.

The water maze spatial learning test took place over a period of seven days. The first five days constituted the training phase in which the rats learned where the hidden platform is. Immediately $1 \mathrm{~h}$ after the last training session on the 5 th day, the first probe trial followed in order to assess recent memory. Rats were again trained on the 6th day and $24 \mathrm{~h}$ later subjected to a second probe trial to assess remote memory.

The training period consisted of four trials per day, starting the rat at four different positions in the water maze, to avoid left and right navigation to the platform. Each trial began with the rat in the pool facing the sidewalls and ended when the rat found the platform or after $60 \mathrm{~s}$; in both cases the rat was allowed to stay on the platform for 10 s. On the 5th and 7th day a probe trial of $60 \mathrm{~s}$ was performed after the escape platform had been removed. Time spent in the peripheral and target quadrants was analyzed using Ethovision tracking software.

\section{Object Recognition Task}

The apparatus consisted of a square arena measuring $40 \times 40 \mathrm{~cm}$ with $40 \mathrm{~cm}$ high walls. In total 3 different objects were used (A) a glass bottle, (B) a round glass jar and (D) a square plastic drinking bottle. In the first trial (T1) two identical glass bottles were placed in two different corners of the arena and the rat was allowed to explore these objects for $3 \mathrm{~min}$. A rat was always placed in the apparatus facing the wall. After T1 the rat was placed back in its home cage and after and intertrial delay of $1 \mathrm{~h}$, the second trial (T2) followed. In T2 one of the glass bottles presented before was replaced by a novel object, a glass jar. Again rats were allowed to explore these two objects for $3 \mathrm{~min}$. The third trial (T3) was evaluated after a 24 retention interval. In this trial the other glass bottle was replaced by a plastic drinking bottle. The duration of T3 was 3 min. Entorhinal cortex and fornix DBS rats were stimulated with $100 \mathrm{~Hz}, 100 \mu \mathrm{A}$ and 100 $\mu$ s 30 min before T1, T2 and T3, while sham rats were only attached to cables and not stimulated.

The time spent exploring each object in T1, T2 and T3 was monitored using a personal computer. Discrimination performance was calculated by subtracting the total 
time at familiar objects from the time at the novel object and dividing it by the total exploration time in T2 or T3. Side bias was determined by the time spent at the left or right object, which was independent of the novel or old position of the object.

\section{Statistical analysis}

Statistical analysis for all behavioral tasks was performed using one-way ANOVA, followed by an LSD post-hoc test. P-values $\leq 0.05$ were considered significant.

\section{RESULTS}

\section{Elevated Plus Maze}

The performance of entorhinal cortex DBS, fornix DBS and sham rats did not differ in the elevated plus maze. In particular, time spent in center, open or closed arms was similar between the groups (all F's $<1.377$, n.s., Fig. 2).

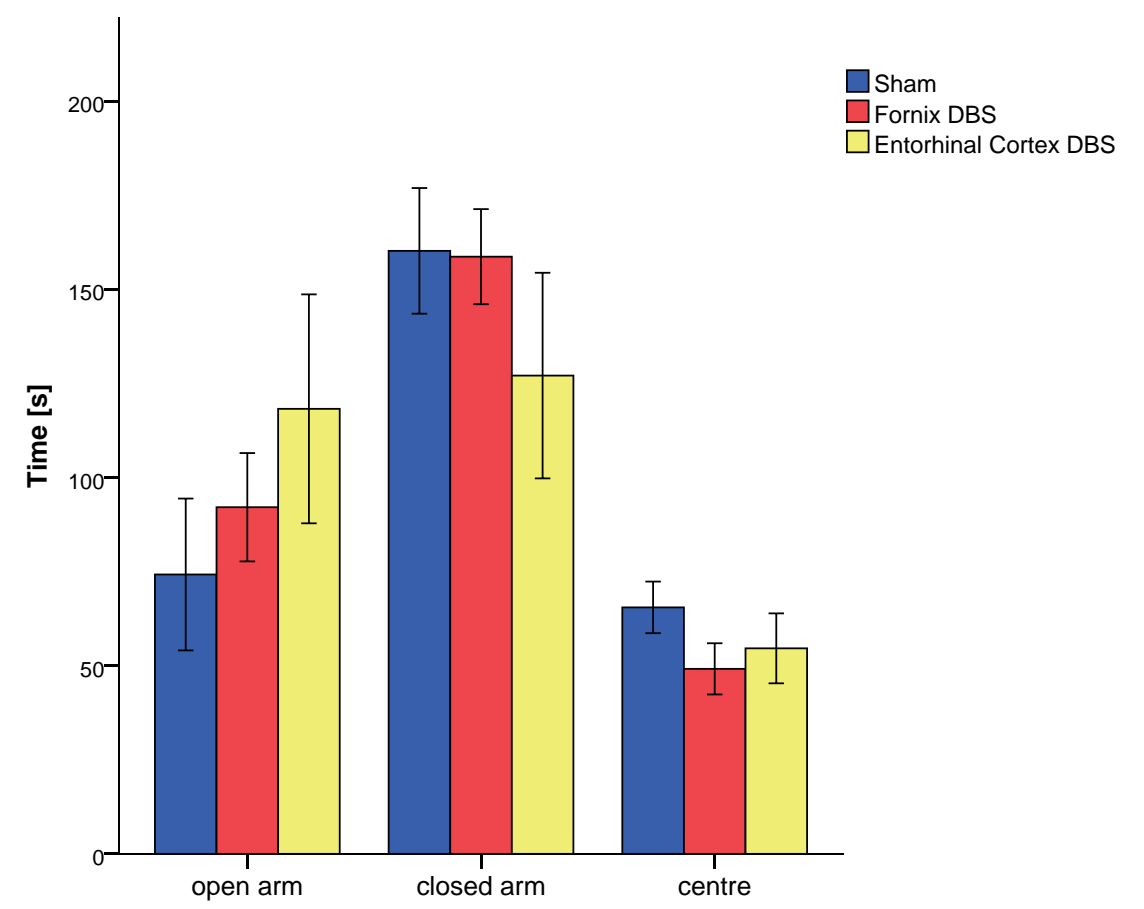

Figure 2: Time spent (in s) in different compartments of the elevated plus maze: open arms, closed arms, center. There was no statistical difference between sham, fornix DBS or entorhinal cortex DBS rats. Data represents mean \pm S.E.M. 


\section{Open Field}

We found no significant difference for time spent in corners, walls and center of the open field between DBS groups and sham operated animals (all F's $<0.624$, n.s., data not shown). The total distance moved did also not differ between the groups $(\mathrm{F}(2 ., 25)=0.368$, n.s., data not shown $)$.

\section{Morris Water Maze}

Fornix and entorhinal cortex DBS rats reached the target faster than sham animals in the first and third training session (all F's $\geq 4.791, \mathrm{p} \leq 0.02$ ). Fornix DBS animals also learned quicker in the second training session when compared to $\operatorname{sham}(\mathrm{F}(2,28)=5.864$, $\mathrm{p}=0.02$, Fig. 3).

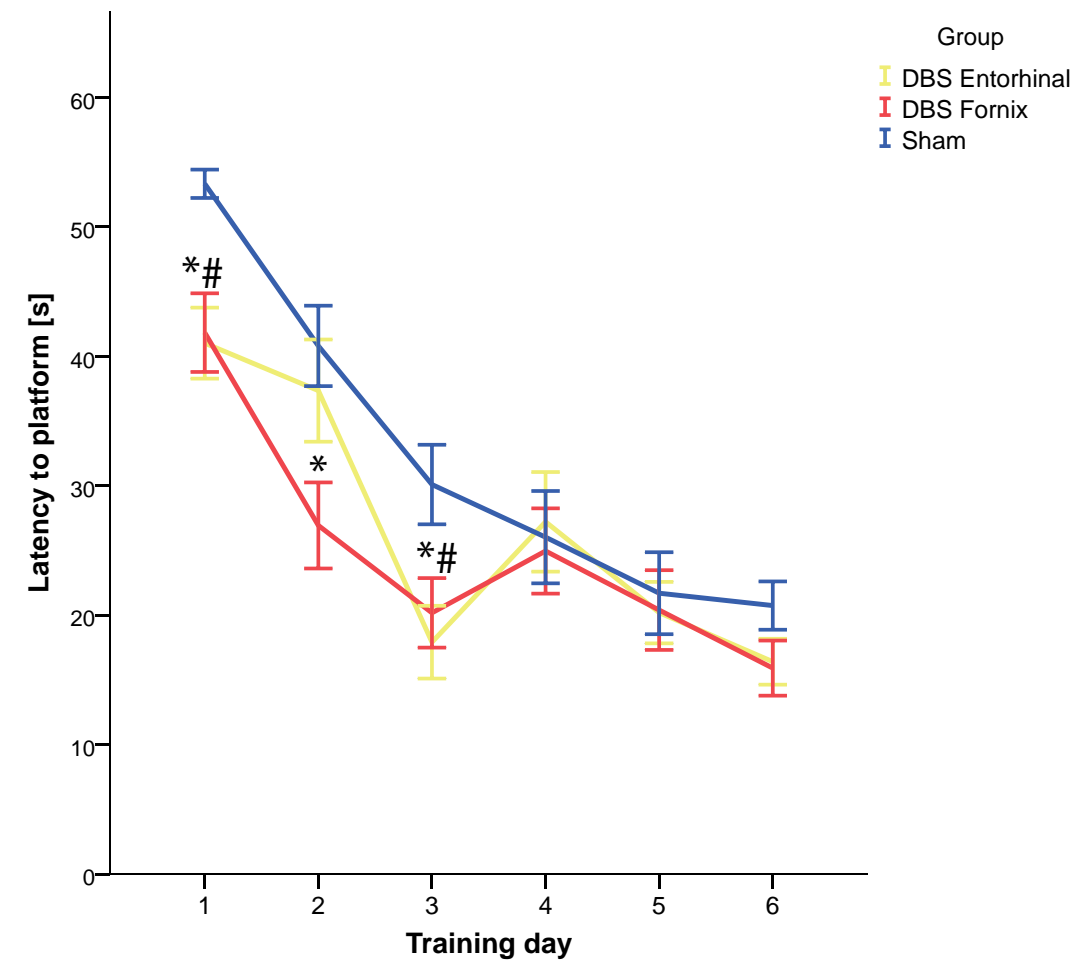

Figure 3: Latency to reach platform in [s] on training day 1-6. Fornix and entorhinal cortex DBS rats acquired the position of the platform faster than sham rats on training days 1-3. * indicates $\mathrm{p}<0.05$ for fornix DBS and \# indicates $\mathrm{p}<0.05$ for entorhinal cortex DBS rats. Data are represented as mean \pm S.E.M. 
In the first probe test $1 \mathrm{~h}$ after training only fornix stimulated animals spent significantly more time in the target quadrant when compared to sham $(\mathrm{F}(2,24)=8.252$, $p=0.001$, Fig. 4). The post-hoc test did not reveal statistical significance for entorhinal cortex stimulated animals $(\mathrm{p}=0.06)$. In the second probe test $24 \mathrm{~h}$ after training, there was no difference for time spent in target quadrant between DBS animals and sham $(\mathrm{F}(2,25)=3.066$, n.s. $)$. Entorhinal cortex stimulated animals spent significantly more time in the quadrant opposing the target quadrant $(\mathrm{F}(2,25)=3.851, \mathrm{p}=0.03$, data not shown).

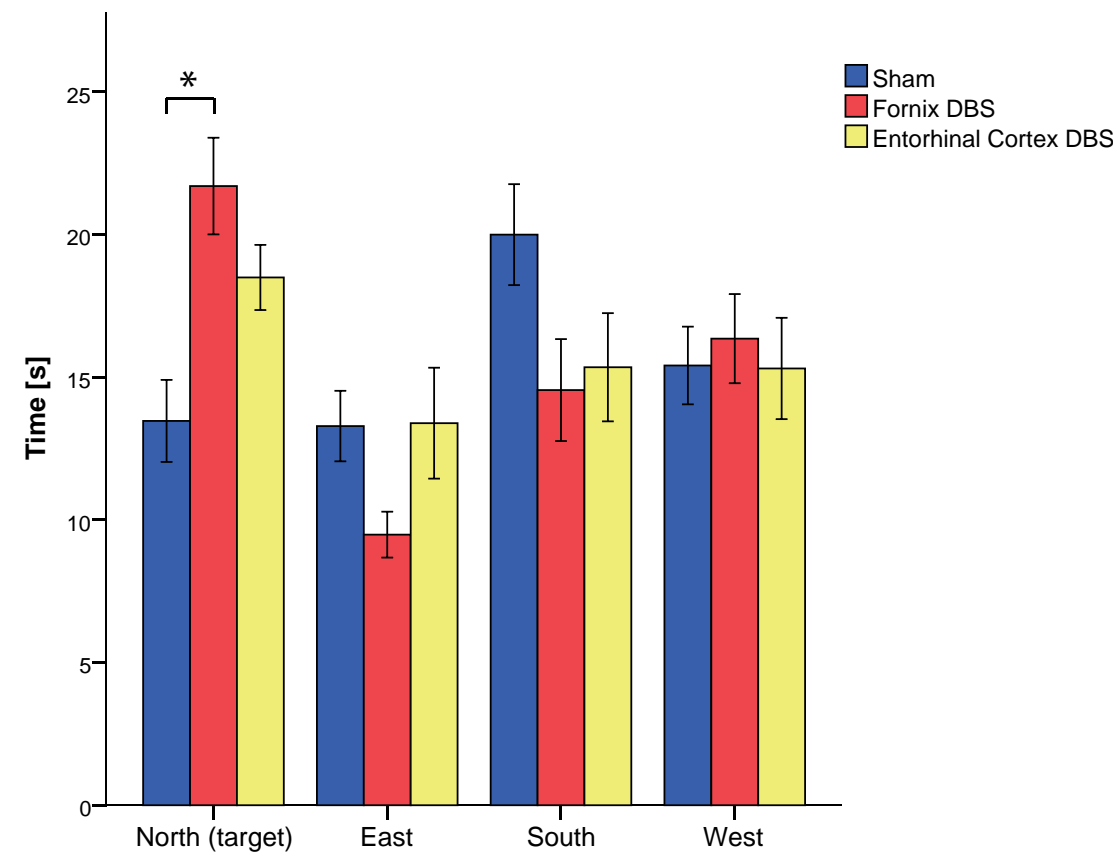

Figure 4: Time spent (in s) in different zones of the water maze after a $1 \mathrm{~h}$ retention interval. Fornix DBS rats spent significantly more time in the target quadrant when compared to sham. ${ }^{*}$ indicates $\mathrm{p}<0.05$. Data represents mean \pm S.E.M.

\section{Object Recognition Task}

After the $1 \mathrm{~h}$ retention interval we found no differences between DBS groups and sham $(\mathrm{F}(2,25)=0.616$, n.s. $)$. At the $24 \mathrm{~h}$ retention interval only entorhinal cortex DBS rats showed improved memory performance when compared to sham. $(\mathrm{F}(2,23)=3.999$, $\mathrm{p}=0.01$, Fig. 5). 


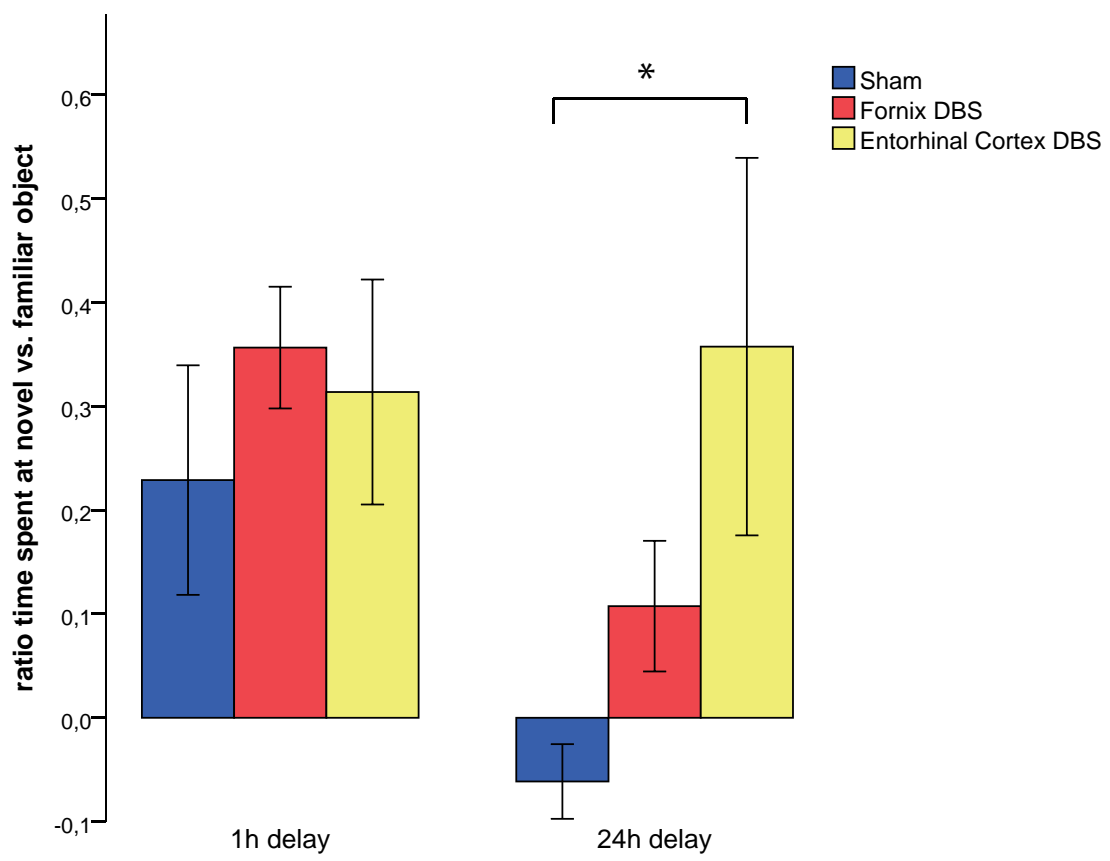

Figure 5: Ratio time spent at novel vs. familiar object for sham, fornix DBS and entorhinal cortex DBS groups. At the $1 \mathrm{~h}$ retention interval there was no significant difference between the groups. All animals show intact recognition memory. At the $24 \mathrm{~h}$ retention interval only entorhinal cortex DBS rats spent significantly more time at the novel object when compared to sham. ${ }^{*}$ indicates $\mathrm{p}<0.05$. Data represents mean \pm S.E.M.

\section{DISCUSSION}

In an aged animal model, DBS of the fornix and entorhinal cortex resulted in beneficial memory effects in different domains. DBS of the fornix restored impaired spatial memory performance in the water maze, when compared to sham treated animals and animals with entorhinal cortex DBS. In particular, rats with fornix DBS spent significantly more time in the target quadrant when the probe trial was carried out immediately after the last training session. Thus, DBS of the fornix improved short-term memory. Contrary to this, DBS of the entorhinal cortex improved recognition memory in the ORT after a 24 hour delay.

The fornix carries numerous projections to and from the hippocampal formation. Close to the anterior commissure, the fornix divides into a precommissural and postcommissural branch. The precommissural fornix innervates the prefrontal cortex and the ventral striatum, while the postcommissural fornix either connects to the anterior thalamus directly or indirectly through the mammillary bodies [23]. Lesions to the fornix have been shown to affect spatial memory [23-25], while recognition memory 
has been spared [24]. Previously, DBS of the fornix has been shown to improve spatial memory in an experimental rat model of dementia [9]. More specifically, fornix DBS was able to restore scopolamine-induced amnesia in the object location task when a combination of certain stimulation parameters was used. Beneficial effects were observed with acute stimulations of $10 \mathrm{~Hz}$ and $200 \mu \mathrm{A}$ or $100 \mathrm{~Hz}$ and $100 \mu \mathrm{A}$ during the trials [9]. Here, we demonstrated that spatial memory deficits naturally occurring through aging might also be improved through fornix DBS.

The entorhinal cortex has strong reciprocal connections with the hippocampal formation and constitutes the major gateway between the hippocampal formation and the neocortex. In fact, the entorhinal cortex has been found to be one of the first structures to degenerate in $\mathrm{AD}$ [26]. Several studies have reported a role of the entorhinal cortex in spatial memory. It was reported that the entorhinal cortex contains a spatial map, consisting of grid cells, which contribute to spatiotemporal orientation during behavior [27]. Previously, it was found that entorhinal cortex DBS of 1 hour at $130 \mathrm{~Hz}, 90 \mu \mathrm{s}$ pulse width and $50 \mu \mathrm{A}$ in mice also showed enhanced spatial memory performance in the water maze. Six and a half weeks after stimulation, behavioral improvement was accompanied by an increase in BrdU positive cells in the dentate gyrus, a hippocampal structure maintaining strong connections with the entorhinal cortex [12]. In the present study we did not find any significant spatial memory improvement in the water maze, neither at the immediate nor at the remote retention interval when stimulating with $100 \mathrm{~Hz}, 100 \mu$ s pulse width and $100 \mu \mathrm{A}$. It might be possible that the applied current density of $100 \mu \mathrm{A}$ was too high, because the current hypothesis states that low current applied in the entorhinal cortex has an effect on neighboring axons, thus activating fibers of the performant path, which in turn induce neurogenesis in the dentate gyrus [17]. Another possibility is that the $24 \mathrm{~h}$ retention interval was not sufficient to produce effects on spatial memory. In the aforementioned study, mice were tested in the water maze six and a half weeks after stimulation, allowing the maturation of new-born neurons [12].

Contrary to this, we found enhanced recognition memory following entorhinal cortex DBS at a $24 \mathrm{~h}$ retention interval in the object recognition task. There was no difference between the groups at the 1 h retention interval, which is commonly used to test short-term memory. These findings suggest that recognition short-term memory is intact in all groups. The $24 \mathrm{~h}$ rentention interval is used to test natural forgetting, since young and healthy rats usually do not discriminate anymore between novel and familiar object after such an interval [28]. Here, we found that entorhinal cortex DBS was able to reverse natural forgetting. Although most studies, which lesioned the entorhinal cortex found impaired spatial memory, some also reported (mild) deficits in recognition memory $[29,30]$. In fact, one has to distinguish different roles of the medial and lateral entorhinal cortex. The medial entorhinal cortex contributes to spatial processing and 
the lateral to non-spatial memory [31]. Besides the hippocampus, it has been suggested that the lateral entorhinal cortex is connected to the perirhinal and postrhinal cortices [32]. The perirhinal and postrhinal cortices have been shown to play a strong role in recognition memory $[33,34]$. Therefore, it might be possible that entorhinal cortex DBS activated the rhinal cortices and thereby induced enhanced recognition memory.

Taken together, these results suggest that the fornix and entorhinal cortex are employed in different memory domains. The fornix seems to play a major role in spatial memory and the entorhinal cortex in recognition memory.

\section{ACKNOWLEDGEMENTS}

This study was funded by the Singapore Lee Kuan Yew Research Fellowship (M4080846.080). 


\section{REFERENCES}

1. Alzheimer's A. 2014 Alzheimer's disease facts and figures. Alzheimer's \& dementia : the journal of the Alzheimer's Association. 2014;10(2):e47-92.

2. Thies W, Bleiler L. 2011 Alzheimer's disease facts and figures. Alzheimers Dement. 2011;7(2):208-44. Epub 2011/03/19. eng.

3. Rosini M, Simoni E, Bartolini M, Cavalli A, Ceccarini L, Pascu N, et al. Inhibition of acetylcholinesterase, beta-amyloid aggregation, and NMDA receptors in Alzheimer's disease: a promising direction for the multi-target-directed ligands gold rush. Journal of medicinal chemistry. 2008;51(15):4381-4.

4. Qaseem A, Snow V, Cross JT, Forciea MA, Hopkins R, Shekelle P, et al. Current Pharmacologic Treatment of Dementia: A Clinical Practice Guideline from the American College of Physicians and the American Academy of Family Physicians. Annals of Internal Medicine. 2008;148(5):370-8.

5. Kringelbach ML, Jenkinson N, Owen SL, Aziz TZ. Translational principles of deep brain stimulation. Nature reviews Neuroscience. 2007;8(8):623-35.

6. Hamani C, Temel Y. Deep Brain Stimulation for Psychiatric Disease: Contributions and Validity of Animal Models. Sci Transl Med. 2012;4(142):142rv8.

7. Fontaine D, Deudon A, Lemaire JJ, Razzouk M, Viau P, Darcourt J, et al. Symptomatic treatment of memory decline in Alzheimer's disease by deep brain stimulation: a feasibility study. J Alzheimers Dis. 2013;34(1):315-23. Epub 2012/11/22. eng.

8. Hamani C, McAndrews MP, Cohn M, Oh M, Zumsteg D, Shapiro CM, et al. Memory enhancement induced by hypothalamic/fornix deep brain stimulation. Annals of Neurology. 2008;63(1):119-23.

9. Hescham S, Lim LW, Jahanshahi A, Steinbusch HW, Prickaerts J, Blokland A, et al. Deep brain stimulation of the forniceal area enhances memory functions in experimental dementia: the role of stimulation parameters. Brain Stimul. 2013;6(1):72-7. Epub 2012/03/13. eng.

10. Laxton AW, Tang-Wai DF, McAndrews MP, Zumsteg D, Wennberg R, Keren R, et al. A phase I trial of deep brain stimulation of memory circuits in Alzheimer's disease. Annals of Neurology. 2010;68(4):521-34.

11. Smith GS, Laxton AW, Tang-Wai DF, McAndrews MP, Diaconescu AO, Workman CI, et al. Increased Cerebral Metabolism After 1 Year of Deep Brain Stimulation in Alzheimer DiseaseIncreased Cerebral Metabolism After 1 Year of DBS. Arch Neurol. 2012:1-8.

12. Stone SSD, Teixeira CM, DeVito LM, Zaslavsky K, Josselyn SA, Lozano AM, et al. Stimulation of Entorhinal Cortex Promotes Adult Neurogenesis and Facilitates Spatial Memory. The Journal of Neuroscience. 2011;31(38):13469-84.

13. Suthana N, Haneef Z, Stern J, Mukamel R, Behnke E, Knowlton B, et al. Memory Enhancement and Deep-Brain Stimulation of the Entorhinal Area. New England Journal of Medicine. 2012;366(6):50210.

14. Freund HJ, Kuhn J, Lenartz D, Mai JK, Schnell T, Klosterkoetter J, et al. Cognitive functions in a patient with Parkinson-dementia syndrome undergoing deep brain stimulation. Arch Neurol. 2009;66(6):781-5. Epub 2009/06/10. eng.

15. Kuhn J, Hardenacke K, Lenartz D, Gruendler T, Ullsperger M, Bartsch C, et al. Deep brain stimulation of the nucleus basalis of Meynert in Alzheimer's dementia. Molecular psychiatry. 2014.

16. Turnbull IM, McGeer PL, Beattie L, Calne D, Pate B. Stimulation of the basal nucleus of Meynert in senile dementia of Alzheimer's type. A preliminary report. Appl Neurophysiol. 1985;48(1-6):216-21. Epub 1985/01/01. eng.

17. Hescham S, Lim L, Jahanshahi A, Blokland A, Temel Y. Deep brain stimulation in dementia-related disorders. Neurosci Biobehav Rev. 2013;37(10 Pt 2):2666-75. Epub 2013/09/26. eng. 
18. Temel Y, Tan S, Vlamings R, Sesia T, Lim LW, Lardeux S, et al. Cognitive and limbic effects of deep brain stimulation in preclinical studies. Front Biosci. 2009;14:1891-901. Epub 2009/03/11. eng.

19. Sengupta P. The Laboratory Rat: Relating Its Age With Human's. International Journal of Preventive Medicine. 2013;4(6):624-30.

20. Paxinos G, Watson C. The rat brain in stereotaxic coordinates: hard cover edition: Academic press; 2006.

21. Tan S, Vlamings R, Lim L, Sesia T, Janssen ML, Steinbusch HW, et al. Experimental deep brain stimulation in animal models. Neurosurgery. 2010;67(4):1073-9. Epub 2010/10/01. eng.

22. Temel Y, Boothman LJ, Blokland A, Magill PJ, Steinbusch HW, Visser-Vandewalle V, et al. Inhibition of 5-HT neuron activity and induction of depressive-like behavior by high-frequency stimulation of the subthalamic nucleus. Proceedings of the National Academy of Sciences of the United States of America. 2007;104(43):17087-92.

23. Vann SD, Erichsen JT, O'Mara SM, Aggleton JP. Selective disconnection of the hippocampal formation projections to the mammillary bodies produces only mild deficits on spatial memory tasks: implications for fornix function. Hippocampus. 2011;21(9):945-57.

24. Ennaceur A, Neave N, Aggleton JP. Spontaneous object recognition and object location memory in rats: the effects of lesions in the cingulate cortices, the medial prefrontal cortex, the cingulum bundle and the fornix. Experimental Brain Research. 1997;113(3):509-19.

25. Dumont JR, Amin E, Wright NF, Dillingham CM, Aggleton JP. The impact of fornix lesions in rats on spatial learning tasks sensitive to anterior thalamic and hippocampal damage. Behav Brain Res. 2015;278(0):360-74.

26. Khan UA, Liu L, Provenzano FA, Berman DE, Profaci CP, Sloan R, et al. Molecular drivers and cortical spread of lateral entorhinal cortex dysfunction in preclinical Alzheimer's disease. Nat Neurosci. 2014;17(2):304-11.

27. Hafting T, Fyhn M, Molden S, Moser M-B, Moser EI. Microstructure of a spatial map in the entorhinal cortex. Nature. 2005;436(7052):801-6.

28. Akkerman S, Blokland A, Reneerkens O, van Goethem NP, Bollen E, Gijselaers HJ, et al. Object recognition testing: methodological considerations on exploration and discrimination measures. Behav Brain Res. 2012;232(2):335-47.

29. Galani R, Weiss I, Cassel JC, Kelche C. Spatial memory, habituation, and reactions to spatial and nonspatial changes in rats with selective lesions of the hippocampus, the entorhinal cortex or the subiculum. Behav Brain Res. 1998;96(1-2):1-12.

30. Parron C, Save E. Comparison of the effects of entorhinal and retrosplenial cortical lesions on habituation, reaction to spatial and non-spatial changes during object exploration in the rat. Neurobiology of Learning and Memory. 2004;82(1):1-11.

31. Hunsaker MR, Chen V, Tran GT, Kesner RP. The medial and lateral entorhinal cortex both contribute to contextual and item recognition memory: A test of the binding ofitems and context model. Hippocampus. 2013;23(5):380-91.

32. Naber PA, Witter MP, Lopez da Silva FH. Perirhinal cortex input to the hippocampus in the rat: evidence for parallel pathways, both direct and indirect. A combined physiological and anatomical study. Eur J Neurosci. 1999;11(11):4119-33.

33. Ennaceur A, Neave N, Aggleton JP. Neurotoxic lesions of the perirhinal cortex do not mimic the behavioural effects of fornix transection in the rat. Behav Brain Res. 1996;80(1-2):9-25.

34. Dickerson BC, Eichenbaum H. The episodic memory system: neurocircuitry and disorders. Neuropsychopharmacology : official publication of the American College of Neuropsychopharmacology. 2010;35(1):86-104. 




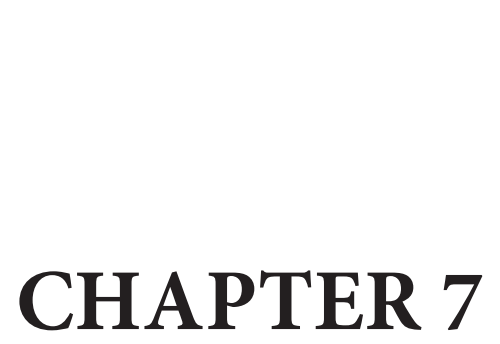

BEHAVIORAL EFFECTS OF DEEP BRAIN STIMULATION OF DIFFERENT AREAS OF THE PAPEZ CIRCUIT ON MEMORY- AND ANXIETY-RELATED FUNCTIONS

Sarah Hescham, Ali Jahanshahi, Céline Meriaux, Lee Wei Lim, Arjan Blokland, Yasin Temel 


\section{ABSTRACT}

Deep brain stimulation (DBS) has gained interest as a potential therapy for advanced treatment-resistant dementia. However, possible targets for DBS and the optimal stimulation parameters are not yet clear. Here, we compared the effects of DBS of the CA1 sub-region of the hippocampus, mammillothalamic tract, anterior thalamic nucleus and entorhinal cortex in an experimental rat model of dementia. Rats with scopolamine-induced amnesia were assessed in the object location task with different DBS parameters. Moreover, anxiety-related side effects were evaluated in the elevated zero maze and open field. After sacrifice, we applied c-Fos immunohistochemistry to assess which memory-related regions were affected by DBS. When comparing all structures, DBS of the entorhinal cortex and CA1 sub-region was able to restore memory loss when a specific set of stimulation parameters was used. No anxiety-related side effects were found following DBS. The beneficial behavioral performance of CA1 DBS rats was accompanied with an activation of cells in the anterior cingulate gyrus. Therefore, we conclude that acute CA1 DBS restores memory loss possibly through improved attentional and cognitive processes in the limbic cortex. 


\section{INTRODUCTION}

Dementia, is a debilitating neurological condition with deterioration in more than one domain of cognitive function. The most prevalent cause of dementia is Alzheimer's disease (AD), accounting for $50-80 \%$ of all cases. In the United States, $9.7 \%$ of the population over 70 years of age, on average, suffers from AD [1]. In Europe the agestandardized prevalence of people aged 65 years or older of population-based studies suggests that $4.4 \%$ is affected [2]. Symptoms include loss of memory, problems with communicating and reasoning, as well as changes in personality and behavior. After diagnosis the expected life span is approximately seven years [3]. Whilst there have been advances for symptomatic treatments for $\mathrm{AD}[4,5]$, there are currently neither cures nor treatments that delay or reverse the effects of $\mathrm{AD}$. Thus, care and pharmacological interventions for dementia are mainly of palliative nature $[6,7]$.

Against this background of limited progress in dementia treatment, researchers have been exploring non-drug based therapies as alternative treatment strategies to reduce or delay the progression of memory loss in $\mathrm{AD}$. One such treatment is deep brain stimulation (DBS). The basic principle of modern DBS is the use of implanted electrodes connected to an internal pulse generator to deliver electrical stimuli to specific brain regions $[8,9]$. This method has mainly been applied in the field of Parkinson's disease in the last two decades, but has been found to produce therapeutic effects in a wide range of neurological [10-12] and psychiatric diseases [13-16]. In line with these developments, evidence from recent preclinical and clinical case studies suggests that DBS of the fornix, entorhinal cortex and nucleus basalis of Meynert might have memory improving properties [17-22].

The rationale for choice of brain regions to improve memory functions in dementia using DBS is based on the classical circuit of Papez [23]. In this circuit, memory-related information flows through the perforant pathway from the entorhinal cortex to fields of the hippocampal formation, including the dentate gyrus, subiculum, CA1 and CA3. The information is then carried through the fornix to the mammillary bodies and thereafter to the anterior nucleus of thalamus via the mammillothalamic tract. The anterior nucleus of the thalamus in turn projects to the cingulate gyrus, and the memory circuit is completed by projections of the cingulate gyrus to the entorhinal cortex of the parahippocampal region [23].

Much of the above circuitry makes a relevant target for DBS. Although we already reported beneficial memory effects following fornix DBS [19], we wanted to explore the applicability and efficacy of other target regions within the circuit of Papez. Therefore, we compared the memory enhancing effects of DBS of the CA1 sub-region of hippocampus, mammillothalamic tract, anterior nucleus of thalamus and entorhinal cortex in a spatial memory task and several anxiety tasks. In addition, we mapped memory- 
related brain regions affected by DBS using early the immediate gene c-Fos (K-25). C-Fos is expressed when neurons fire action potential and can be even used to map long-term activation. In particular, we investigated areas related to memory processes i.e. the medial prefrontal cortex and hippocampus.

\section{MATERIALS AND METHODS}

\section{Subjects}

Sprague Dawley (SD) rats from Charles River (Sulzfeld, Germany) were used, their weight ranging between 300-350 g at the time of surgery. The temperature of the colony room was maintained at a temperature of $21 \pm 1{ }^{\circ} \mathrm{C}$ and rats were individually housed in Makrolon $^{\text {Tw }}$ cages with rat chow and water available ad libitum. Furthermore, the rats were exposed to a reversed 12:12 h light dark cycle. All experimental animal procedures were executed during the dark phase under red light.

\section{Experimental groups}

Rats were randomly assigned to one of the following experimental groups: Sham $(\mathrm{n}=11)$, CA1 DBS $(\mathrm{n}=10)$, mammillothalamic tract DBS $(\mathrm{n}=13)$, anterior thalamic nucleus DBS $(n=14)$ and entorhinal cortex DBS $(n=15)$.

\section{Surgical procedure}

DBS electrodes were implanted bilaterally in the target areas using a rodent stereotactic apparatus (Stoelting, Wood Dale, IL, USA, model 51653). Isoflurane (IsoFlo ${ }^{\oplus}$, Abbott Laboratories Ltd, Berkshire, Great Britain) was used as inhalation anesthesia. After exposure of the rat skull, two burr holes were made either at the level of the CA1 (coordinates from bregma according to the rat brain atlas of Paxinos and Watson [24]: AP: -3.6 mm; ML: $1.8 \mathrm{~mm}$; DV: $-2.6 \mathrm{~mm}$ ), mammillothalamic tract (AP:-1.8 mm; ML: $1 \mathrm{~mm}$; DV: $-6.2 \mathrm{~mm}$ ), anterior thalamic nucleus (AP: $-1.6 \mathrm{~mm}$; ML: $1.5 \mathrm{~mm}$; DV: $-5.2 \mathrm{~mm}$ ), or entorhinal cortex (AP:-6.7 mm; ML: $4 \mathrm{~mm}$; DV: $-8 \mathrm{~mm}$ ). More detailed information about electrodes and surgical procedures is given in previous publications from our group $[25,26]$. Shams also underwent electrode implantation in the same brain targets, but were never stimulated. All rats were given a 2 week recovery period after surgery.

\section{Drugs}

Scopolamine hydrobromide (Acros Organics BVBA, Geel, Belgium) was dissolved in vehicle solution (saline; $0.9 \% \mathrm{NaCl}$ ) and injected intraperitoneally at a dose of $0.1 \mathrm{mg}$ / $\mathrm{kg}$ (in $1 \mathrm{ml} / \mathrm{kg}$ ) $30 \mathrm{~min}$ before the first trial in 6 consecutive sessions of the Object 
Location Task (OLT). The chosen dose of scopolamine has been described to affect spatial and recognition memory [27].

\section{Deep brain stimulation}

The following experimental conditions were tested: (i) saline injection with attachment of the stimulation cable (no stimulation) and (ii) scopolamine injection with DBS at various amplitudes $(50 \mu \mathrm{A}, 100 \mu \mathrm{A}$ and $200 \mu \mathrm{A})$ and frequencies $(100 \mathrm{~Hz}$ or $10 \mathrm{~Hz})$ at a pulse width of $100 \mu$ s. Stimulation was bipolar and monophasic in all cases. A randomized stimulation paradigm was applied between animals on the different testing days of the OLT. Stimulation was performed using a World Precision Instrument digital stimulator (DS8000, WPI, Berlin, Germany) and two stimulus isolators (DLS100, WPI, Berlin, Germany). Each animal was given a $24 \mathrm{~h}$ stimulation-off period between testing sessions. Prior to perfusion, the animals were stimulated for $1 \mathrm{~h}$ and were given $1 \mathrm{~h}$ of rest before sacrifice in order to observe expression of c-Fos [28].

\section{Behavioral testing}

\section{Object Location Task}

A full description of the OLT can be found in our previous publication [19]. In short, rats were placed in a circular arena $(83 \mathrm{~cm}$ diameter) with $40 \mathrm{~cm}$ high walls. Half of the surrounding walls were made of grey polyvinyl chloride and the other half of transparent polyvinyl chloride. In total four different sets of objects were used (A) a standard $1 \mathrm{~L}$ glass bottle (diameter $10 \mathrm{~cm}$, height $22 \mathrm{~cm}$ ) filled with water, (B) plastic cups (diameter $7.8 \mathrm{~cm}$, height $9 \mathrm{~cm}$ ), (C) aluminum containers $(7.5 \mathrm{~cm}, 7.5 \mathrm{~cm}, 10 \mathrm{~cm}$ ) and (D) ceramic pyramids $(11.5 \mathrm{~cm} \times 11.5 \mathrm{~cm} \times 9.5 \mathrm{~cm})$.

Objects were presented in a counterbalanced order to avoid preferences for particular objects or locations. In the first trial (T1) two identical objects were placed in a symmetrical position in the center of the arena and the rat was allowed to explore these objects for $3 \mathrm{~min}$. After an inter-trial delay of $90 \mathrm{~min}$, the second trial (T2) followed. In $\mathrm{T} 2$ one of the objects presented before was moved to a novel position (either left or right object was moved $10 \mathrm{~cm}$ to the front or to the back in a counterbalanced order). Using different sets of objects on each day per animal, as well as novel positions in T2 prevented habituation effects.

Two pre-tests with vehicle injections but without stimulation were carried out in order to establish a baseline. Following this, rats were given scopolamine injections 30 min before T1. Stimulation started 2 min before placing the rat into the testing arena (for both T1 and T2) and continued while the rats were in the arena for $3 \mathrm{~min}$. Sham rats underwent the same procedures but were not stimulated. 
Exploration time in T1 and T2 was measured manually by pressing keys on a custommade computer program. During T1 and T2 total exploration time was considered as the sum of time spent at both objects. Discrimination performance was calculated by subtracting the total time at objects in old position from the time at object at novel position and dividing it by the total exploration time in T2. Groups, which showed an effect for particular stimulation parameters, were re-tested and the average discrimination performance was used for analysis. Side bias was determined by the time spent at the left or right object, which was independent of the novel or old position of the object.

\section{Open Field}

The Open Field Test (OFT) consisted of a clear Plexiglas square arena measuring 100 $\mathrm{x} 100 \mathrm{~cm}$ with $40 \mathrm{~cm}$ high walls and a dark floor. The centre of $70 \mathrm{x} 70 \mathrm{~cm}$ was demarcated on a computer, leaving a surrounding outer zone of $15 \mathrm{~cm}$ width. DBS with the most optimal stimulation parameters derived from the OLT was applied for each DBS group starting $2 \mathrm{~min}$ before placing the rat into the arena as well as throughout the 5 min trial. Please note, most optimal stimulation parameter refers to the stimulation parameter, which was able to restore scopolamine-induced memory loss in the OLT or which produced the highest discrimination performance. The behavior of each rat was recorded with Ethovision tracking software (Ethovision, Noldus Information Technology, Wageningen, The Netherlands).

\section{Elevated Zero Maze}

The Elevated Zero Maze (EZM), consists of a circular runway $(98 \mathrm{~cm}$ diameter, $10 \mathrm{~cm}$ path width, $70 \mathrm{~cm}$ above floor level), which was equally divided into two open and two parts enclosed with high side walls $(50 \mathrm{~cm})$. DBS was again applied using most optimal stimulation parameters from the OLT. Rats were stimulated 2 min before as well as throughout the $5 \mathrm{~min}$ trial. Time spent in the open and enclosed parts was recorded with Ethovision tracking software.

\section{Tissue collection}

At the end of the experiments, the rats were overdosed with pentobarbital (120-180 mg/kg I.P., Pharmacy Faculty of Veterinary Science, Utrecht, The Netherlands) and perfused, first with tyrode solution and then Somogyi fixative solution (4\% paraformaldehyde, picric acid, PBS, glutaraldehyde). Brains were removed and placed in fresh fixative (identical composition as Somogyi solution, but lacking glutaraldehyde) at $4^{\circ} \mathrm{C}$ for $2 \mathrm{~h}$. Subsequently brains were transferred to $1 \% \mathrm{NaN}_{3}$ at $4^{\circ} \mathrm{C}$ for long-term storage.

For the sectioning procedure, brains were embedded in $10 \%$ gelatin from porcine skin (Sigma-Aldrich, Zwijndrecht, The Netherlands), and then cut into $30 \mu \mathrm{m}$ slices in 
the frontal plane using a vibratome (Leica ${ }^{\circledR}$, Wetzlar, Germany). Slices were immediately transferred into $1 \% \mathrm{NaN}_{3}$ and kept at $4^{\circ} \mathrm{C}$.

\section{Fos immunohistochemistry}

For immunohistochemistry, sections of 6 random animals per group were selected. In brief, sections were incubated overnight with polyclonal rabbit anti-c-Fos (K-25) primary antibody (1:2000; Santa Cruz Biotechnology Inc, Santa Cruz, USA) and then with biotinylated donkey anti-rabbit secondary antibody (1:800; Jackson Immunoresearch Laboratories Inc., Westgrove, USA) followed by avidin-biotin peroxidase complex (1:800, Elite ABC-kit, Vestastatin, Burlingame, CA, USA). The staining was visualized by 3,3'-Diaminobenzidine (DAB) combined with $\mathrm{NiCl} 2$ intensification.

For immunohistochemical analysis, always 2 similar bregma levels were photographed using a U-CMAD-2 digital camera connected to an Olympus AX70 brightfield microscope (analySIS; Imaging System, Münster, Germany) and evaluated with ImageJ (Image J software version 1.38x; NIH, Bethesda, USA). C-Fos cells in the medial prefrontal cortex (Bregma $+3.2 \mathrm{~mm} \pm 0.1 \mathrm{~mm}$ ) and hippocampus (Bregma $-3.3 \mathrm{~mm} \pm 0.1$ $\mathrm{mm}$ ) were counted manually and the percentage increase or decrease when compared to sham was analyzed.

\section{ELECTRODE VERIFICATION}

Sections containing the electrode trajectories from all animals were collected and mounted on gelatin coated glass slides. A standard haematoxylin-eosin staining was carried out before sections were photographed under bright field microscopy.

\section{Statistical analysis}

For the OLT, the total exploration time in T1 was analyzed by a one-way ANOVA followed by a 2 -sided Dunnett post-hoc test. Since the discrimination performance of a sham group can either be slightly positive or negative and this would lead to a Type I or II error, a virtual group was constructed. This statistical approach has been suggested previously $[29,30]$. In short, a virtual group has a mean of zero and a S.E.M. comparable to the experimental groups. Therefore, comparisons to a virtual group have been suggested as the most valid manner to examine discrimination performance of experimental groups [29]. A one-way ANOVA was performed comparing individual groups to the virtual group and one-sided Dunnett's post-hoc test was applied [c.f. 29]. For the OFT and EZM test as well as the immunohistochemical data, a one-way ANOVA was employed, followed by 2 -sided Dunnett post-hoc test, in which individual 
DBS groups were compared to sham. All p-values $<0.05$ for the aforementioned tests were considered significant.

\section{RESULTS}

\section{Histological evaluation of electrode placements}

For CA1, anterior thalamic nucleus and entorhinal cortex, all electrode tips were verified within the target area. We excluded one animal in the mammillothalamic tract group due to incorrect placement of the electrodes. With the current stimulation settings we found no evidence for histological damage as observed by a standard haematoxylin-eosin staining (Fig. 1 and 2).
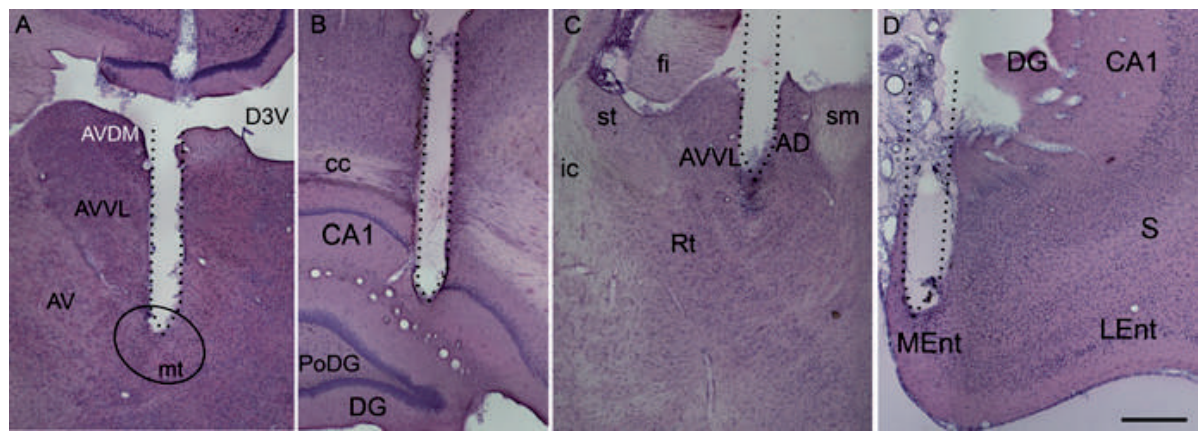

Figure 1: Histological evaluation of electrode tips for mammillothalamic tract (A), CA1 (B), anterior thalamic nucleus (C), and entorhinal cortex (D). Sites were localized by microscopic examination of histologically prepared tissue (bar $=500 \mu \mathrm{m})$. AVDM: anteroventral thalamic nucleus, dorsomedial part; AV: anteroventral thalamic nucleus; AVVL: anteroventral thalamic nucleus, ventrolateral part; mt: mammillothalamic tract; D3V: dorsal third ventricle; cc: corpus callosum; DG: dentate gyrus; PoDG: polymorph layer of dentate gyrus; AD: anterodorsal thalamic nucleus; fi: fimbria; ic: internal capsule; st: stria terminalis; Rt: reticular thalamic nucleus; sm: stria medullaris of the thalamus; S: subiculum; LEnt: lateral entorhinal cortex; MEnt: medial entorhinal cortex.

\section{Object Location Task}

In the OLT, all groups had equal exploration levels in T1 in all sessions when compared to sham. Only entorhinal cortex DBS animals spent significantly less time exploring the objects than sham animals $(\mathrm{F}(4,57)=3.619 ; \mathrm{p}<0.01$; data not shown). However, entorhinal cortex DBS rats still met the requirements of the minimal exploration time (i.e. $10 \mathrm{~s}$ ) by twofold and were therefore considered to be reliable for calculating discrimination performance. When vehicle was injected and stimulation was off, all groups significantly differed from the virtual group $(F(5,68)=3.106$; all p's $\leq 0.02)$. When 

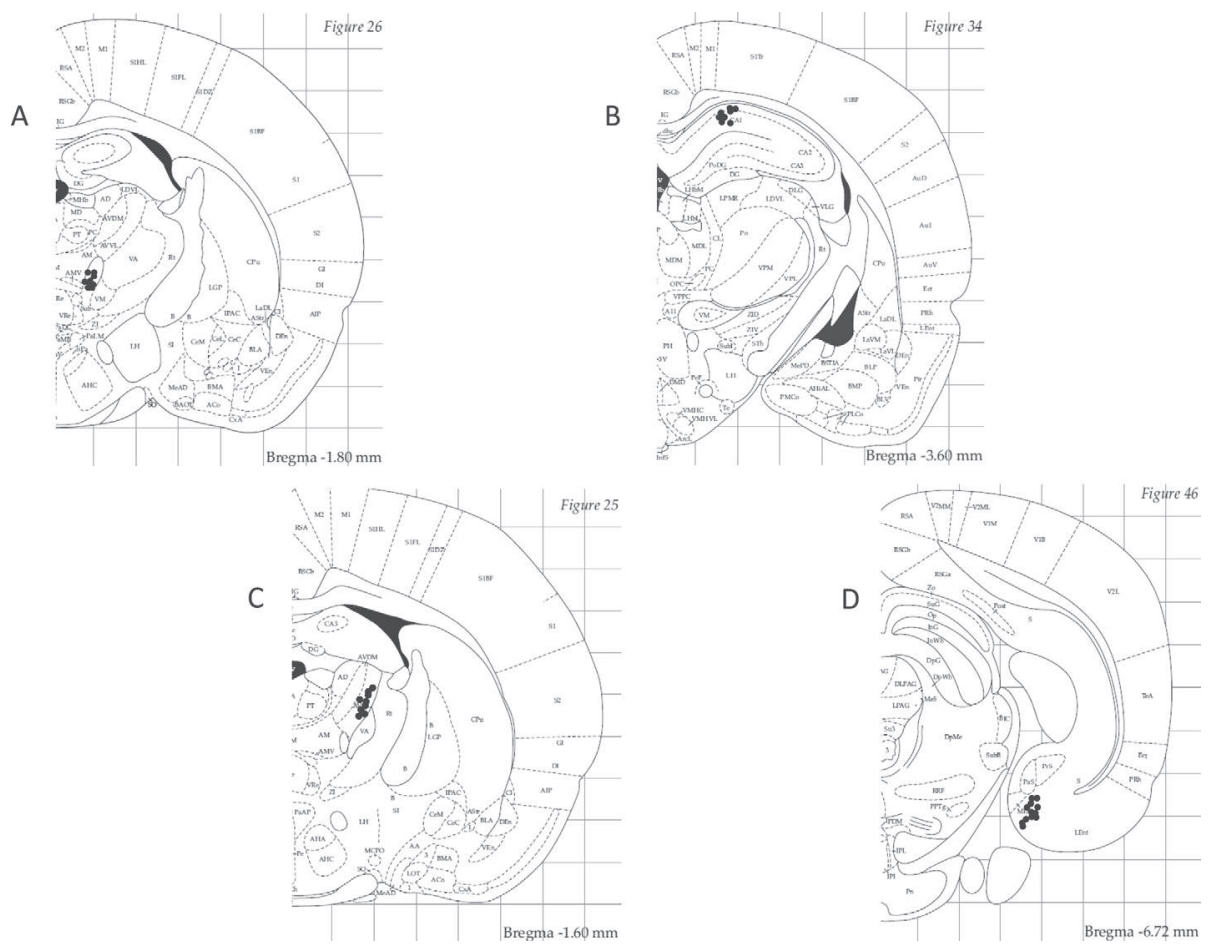

Figure 2: Location of electrode tips in mammillothalamic tract (A), CA1 (B), anterior thalamic nucleus (C), and entorhinal cortex (D) shown schematically in one hemisphere (modified from the atlas of Paxinos and Watson [24]).

scopolamine was injected and DBS groups were stimulated, we found a significant restoration of memory for entorhinal cortex DBS rats at $10 \mathrm{~Hz}$ and $200 \mu \mathrm{A}(\mathrm{F}(5,66)=2.965$; $\mathrm{p}<0.03)$. Although the ANOVA did not show an overall effect between all groups at $100 \mathrm{~Hz}$ and $100 \mu \mathrm{A}$, the post-hoc test revealed significant memory restoration for CA1 DBS rats when compared to the virtual group ( $\mathrm{p}<0.02 ;$ Fig. 3 ).

\section{Open Field and Elevated Zero Maze}

The total distance moved in the OFT was similar between DBS groups and sham $(\mathrm{F}(5,53)=0.608$; n.s.; Table 1$)$. When considering time spent in center or periphery of the OFT, the overall ANOVA indicated a statistical difference between the groups, however the post-hoc test revealed no significant differences between different DBS groups and sham (all F's $\geq 2.516$; n.s.; data not shown). There was also no difference in time spent in the open $(\mathrm{F}(4,51)=0.818$; n.s. $)$ or closed $\operatorname{arm}(\mathrm{F}(4,51)=0.860$; n.s. $)$ of the EZM between the DBS groups and sham (Table 2). 

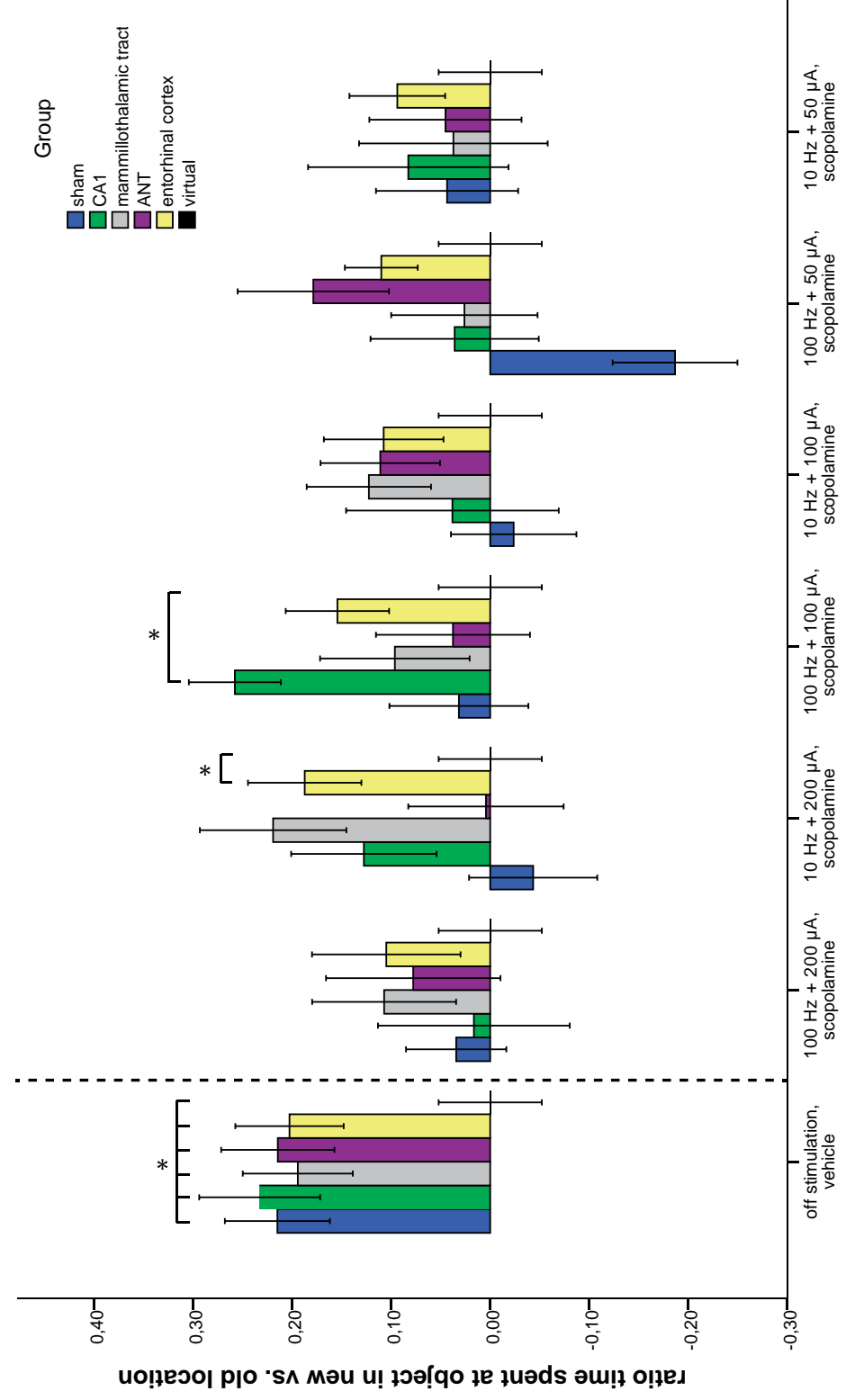

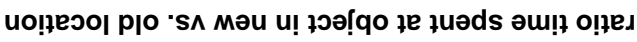

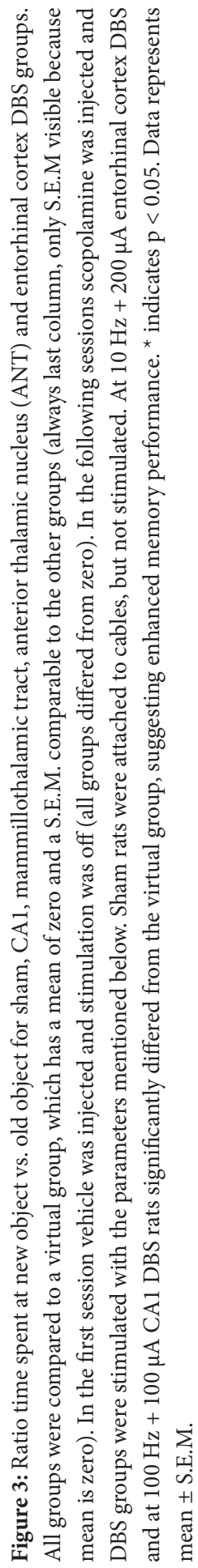


Table 1: Total distance moved (in $\mathrm{cm}$ ) in the open field \pm S.E.M. There was no significant difference between sham and DBS groups. ANT: anterior thalamic nucleus.

\begin{tabular}{ll}
\hline Group & Mean $[\mathrm{cm}] \pm$ S.E.M. \\
\hline Sham & $3653.8 \pm 245.6$ \\
CA1 & $3513.4 \pm 209.2$ \\
Mammillothalamic tract & $3691.8 \pm 297.5$ \\
ANT & $3506.0 \pm 224.9$ \\
Entorhinal cortex & $3888.5 \pm 118.0$ \\
\hline
\end{tabular}

Table 2: Time spent in the open or closed arm of the elevated zero maze in seconds \pm S.E.M. There was no significant difference between sham and DBS groups. ANT: anterior thalamic nucleus.

\begin{tabular}{lll}
\hline Group & Open arm [s] \pm S.E.M. & Closed arm [s] \pm S.E.M. \\
\hline Sham & $146.8 \pm 6.0$ & $153.0 \pm 6.0$ \\
CA1 & $162.5 \pm 10.4$ & $137.3 \pm 10.4$ \\
Mammillothalamic Tract & $150.0 \pm 15.7$ & $149.3 \pm 15.7$ \\
ANT & $172.2 \pm 12.6$ & $126.9 \pm 12.3$ \\
Entorhinal cortex & $158.5 \pm 8.7$ & $138.0 \pm 9.1$ \\
\hline
\end{tabular}

\section{Fos immunohistochemistry}

In the medial prefrontal cortex we observed an increased Fos expression in the cingulate gyrus only for CA1 DBS rats $(F(4,30)=6.966$; $\mathrm{p} \leq 0.001)$. Moreover, we found a significant increase of c-Fos levels in CA1 and mammillothalamic tract DBS animals in the infralimbic $(\mathrm{F}(4,30)=6.433 ; \mathrm{p} \leq 0.015)$ and prelimbic cortex $(\mathrm{F}(4,30)=3.563$, $\mathrm{p} \leq 0.025$; Fig. 4).

In the hippocampus we found an increased c-Fos expression in the CA3 subregion for entorhinal cortex and anterior thalamic nucleus DBS animals when compared to sham $(\mathrm{F}(4,27)=4.293 ; \mathrm{p} \leq 0.01)$. There was no statistical difference between the different DBS groups and sham in the CA1 subregion and dentate gyrus (all F's $\leq 2.409$; n.s.; Fig. 5).

\section{DISCUSSION}

The present study examined the effect of DBS of different targets within the circuit of Papez in a rat model of memory deficit, specifically scopolamine-induced, spatial memory deficit in the OLT. We show that DBS in the CA1 and entorhinal cortex can reverse scopolamine-induced memory deficits. Moreover, we did not observe anxietyrelated side effects of different DBS groups when compared to sham in the EZM and OFT. 

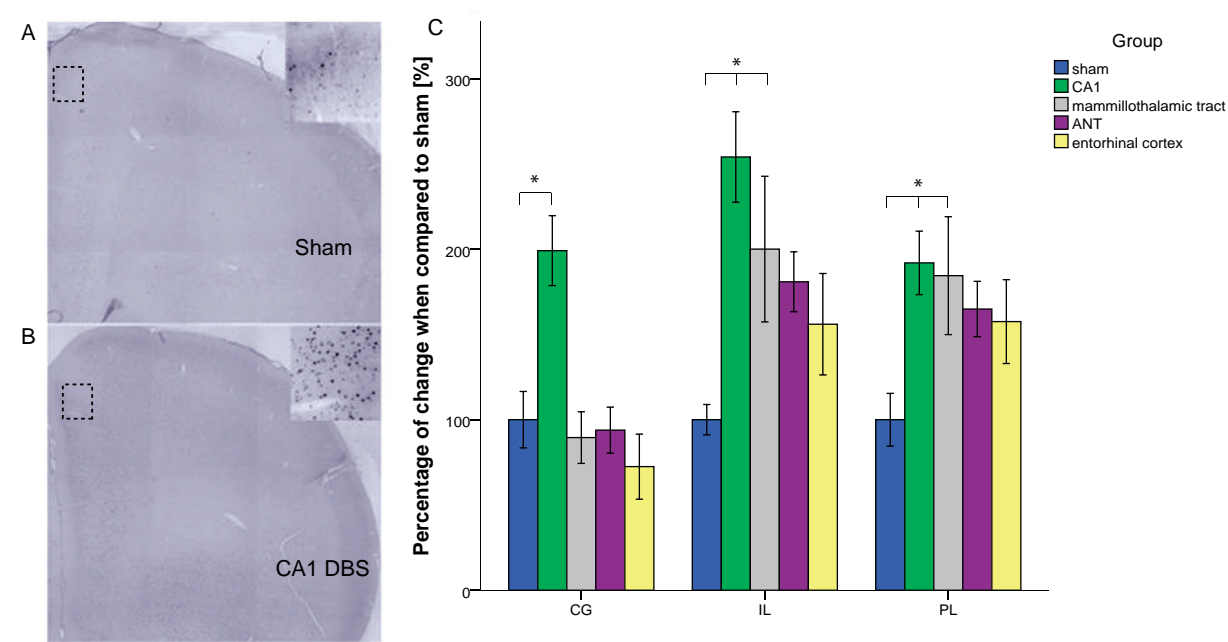

Figure 4: Representative low-power photomicrographs (scale bar $=1000 \mu \mathrm{m}$ ) of coronal brain sections stained for c-Fos (K-25) showing the medial prefrontal cortex of sham (A) and CA1 DBS animals (B). The high-power photomicrograph insets in the upper right corner show the anterior cingulate gyrus (scale bar $=50 \mu \mathrm{m})$. (C) Comparisons were made as percentage increase/decrease with regard to sham. CA1 DBS animals showed increased Fos expression in Cg, whereas CA1 DBS and mammillothalamic tract DBS rats had increased Fos levels in IL and PL. ANT: anterior thalamic nucleus; Cg: cingulate gyrus; IL: infralimbic cortex; PL: prelimbic cortex. ${ }^{*}$ indicates $\mathrm{p}<0.05$. Data represents mean \pm S.E.M.

At present, the evaluation of hippocampal DBS has mainly focused on seizure frequency of epileptic patients [31,32] and little is known about the secondary effects in memory processes. Studies in which neuropsychological tests for memory were included have reported no change in memory scores following hippocampal DBS [33, 34]. Single pulse stimulations of the hippocampus of epileptic patients were even found to impair rather than facilitate memory $[35,36]$. In these studies, it was suggested that stimulation within the hippocampus acutely disrupts formation and recall of recent episodic memory. Here, we found beneficial memory effects following acute CA1 DBS at $100 \mathrm{~Hz}, 100 \mu \mathrm{A}$, and $100 \mu$ s pulse width. This favorable outcome might have been related to the site of stimulation. While in epileptic patients, DBS electrodes are usually not placed in particular subfields, we specifically targeted the CA1 subregion of the hippocampus. The CA1 subregion of the hippocampus is important for spatial memory [37] and is frequently used to study plasticity. It might be possible that DBS of the CA1 subregion induced activity-dependent plastic changes, although this needs further investigation, since we found no evidence for enhanced Fos activity in the CA1 region. Instead, we could link the beneficial memory effects to enhanced neuronal activation in the cingulate gyrus; a structure, which is engaged in attentional processes [38], but also memory consolidation and retrieval [39]. Other regions within the medial prefrontal cortex, namely the infralimbic and prelimbic cortex, exhibited elevated Fos expression 


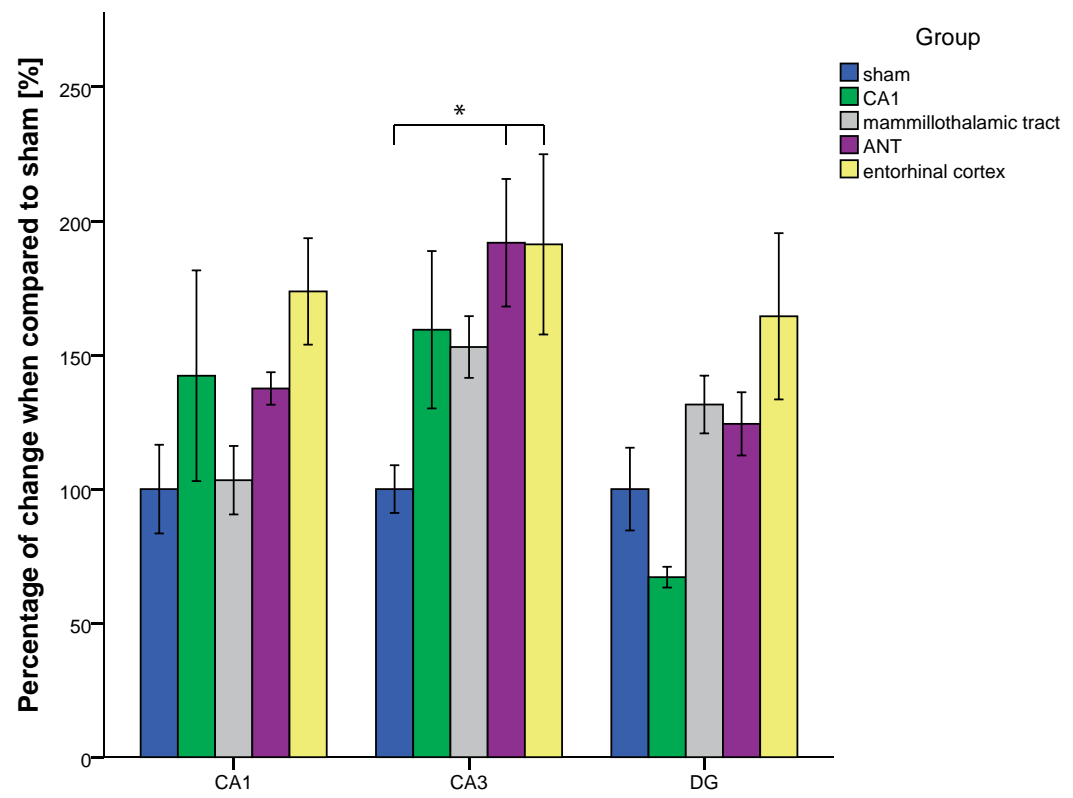

Figure 5: Comparisons of hippocampal c-Fos (K-25) were made as percentage increase/decrease with regard to sham. In the CA3 subregion, entorhinal cortex and anterior thalamic nucleus DBS rats show increased c-Fos expressions. There was no statistical difference between sham and DBS groups in the CA1 and dentate gyrus. ANT: anterior thalamic nucleus. ${ }^{*}$ indicates $\mathrm{p}<0.05$. Data represents mean \pm S.E.M.

for CA1, but also for mammillothalamic tract DBS rats. Since mammillothalamic tract DBS animals did not show memory restoration in the OLT, these results can be interpreted as mere consequence due to stimulation or the activation of cell types, which do not play a role in memory.

We also found restoration of memory loss for entorhinal cortex DBS rats when stimulating with $10 \mathrm{~Hz}$ and $200 \mu \mathrm{A}$. This is in line with findings from a clinical study in which seven pharmacoresistant epilepsy patients were implanted with bilateral DBS electrodes in the entorhinal cortex region [22]. The authors reported that spatial learning was enhanced by DBS in a virtual spatial memory task. Stimulation was acute and at a frequency of $50 \mathrm{~Hz}$. Moreover, an intermittent stimulation paradigm with a cycle of $5 \mathrm{~s}$ on and $5 \mathrm{~s}$ off was applied. The current hypothesis states, that beneficial effects of entorhinal cortex DBS during the learning phase might be associated to theta phase resetting in the hippocampus, allowing the best possible encoding of stimuli [40]. The theta rhythm $(3-8 \mathrm{~Hz})$ can be recorded by electroencephalography (EEG) and has been strongly implicated in mnemonic processing. Stimulus presentation, for example, induces shifts in the phase of hippocampal theta-band oscillations and it has been suggested that resetting theta activity facilitates long-term potentiation [41]. In the present 
study, we were not able to correlate beneficial memory effects of entorhinal cortex DBS to a selective neuronal activation in the hippocampus. In fact, we found increased c-Fos expression in the CA3 subfield of the hippocampus for both, entorhinal cortex and anterior thalamic nucleus DBS rats. The CA3 sub-region plays a role in encoding and retrieval of spatial location sequences [42]. This effect could be related to the improved memory performance of entorhinal cortex stimulated animals, but since DBS of the anterior thalamic nucleus did not reverse the scopolamine-induced memory deficit, these results may again be interpreted as mere stimulation effects, which did not contribute to the memory performance of the rats. There was no difference in c-Fos levels in the CA1 and dentate gyrus between DBS groups and sham.

We could not find beneficial memory effects following mammillothalamic tract or anterior thalamic nucleus DBS with any applied combination of stimulation. While mammillothalamic tract DBS has not changed memory scores in patients [43], anterior thalamic nucleus DBS has shown to disrupt rather than facilitate memory functions in rodents, at least when high current densities are used [44]. DBS was applied to this nucleus for the treatment of severe epilepsy in the SANTE (stimulation of the anterior nucleus of the thalamus for epilepsy) trial (NCT00101933). Although neuropsychological testing showed no group differences in cognitive functions, more participants of the stimulated group subjectively experienced depression and memory problems [45].

In the OFT and EZM, we found no clear evidence for anxiety-related side effects after DBS. It has been shown that structures within the circuit of Papez are involved in different experimental models of anxiety [46]. Although the present study did not reveal anxiogenic effects of DBS in various structures of the Papez circuit, it is our opinion that tests for anxiety should be included when applying DBS to different structures within this circuit in clinical trials [47].

Summarizing, our results suggest that DBS of the CA1 subfield of the hippocampus seems to reverse scopolamine-induced deficits by modulating neuronal activity of the cingulate gyrus. In terms of accessibility in the human brain, however, we foresee challenges in targeting the CA1 subregion for DBS. DBS of the entorhinal cortex did not induce increased neuronal activity in the medial prefrontal cortex or hippocampus, which could be linked to the improved memory performance. Therefore, further studies are indicated to explore circuit-wide effects.

Previously we have shown that fornix DBS is also able to restore memory loss in an experimental rat model of dementia [19]. Fornix DBS was able to reverse scopolamineinduced amnesia independent of frequency, but when high current densities were applied. The fornix consists of myelinated fibers and has been targeted in several clinical trials, which reported beneficial effects on memory $[18,44]$. In a concurrent unpublished study, fornix DBS has shown to influence neuronal activity and neurotransmitter release in the hippocampus. 
Altogether, our conclusions are twofold. Firstly, we have shown that within the circuit of Papez, only DBS of the CA1 subregion, entorhinal cortex and fornix is able to restore spatial memory-related functions. Secondly, however, from a stereotactic point of view, we feel that a strategic target should be chosen for therapy. The hippocampus and entorhinal cortex are large areas in rodents and primates and neuromodulation through a single electrode might be difficult. The fornix, on the other side, consists of a localized bundle of myelinated fibers and has an accessible size in both, rodents and primates. Thus, when considering the circuit of Papez, the fornix might consequently represent a preferred target for DBS therapy in dementia-related disorders.

\section{ACKNOWLEDGEMENTS}

This study was funded by the ISAO. 


\section{REFERENCES}

1. Plassman BL, Langa KM, Fisher GG, Heeringa SG, Weir DR, Ofstedal MB, et al. Prevalence of dementia in the United States: the aging, demographics, and memory study. Neuroepidemiology. 2007;29(1-2):125-32. Epub 2007/11/03. eng.

2. Lobo A, Launer LJ, Fratiglioni L, Andersen K, Di Carlo A, Breteler MM, et al. Prevalence of dementia and major subtypes in Europe: A collaborative study of population-based cohorts. Neurologic Diseases in the Elderly Research Group. Neurology. 2000;54(11 Suppl 5):S4-9. Epub 2000/06/15. eng.

3. Brookmeyer R, Gray S, Kawas C. Projections of Alzheimer's disease in the United States and the public health impact of delaying disease onset. American journal of public health. 1998;88(9):133742. Epub 1998/09/16. eng.

4. Thies W, Bleiler L. 2011 Alzheimer's disease facts and figures. Alzheimers Dement. 2011;7(2):208-44. Epub 2011/03/19. eng.

5. Rosini M, Simoni E, Bartolini M, Cavalli A, Ceccarini L, Pascu N, et al. Inhibition of acetylcholinesterase, beta-amyloid aggregation, and NMDA receptors in Alzheimer's disease: a promising direction for the multi-target-directed ligands gold rush. Journal of medicinal chemistry. 2008;51(15):4381-4.

6. Qaseem A, Snow V, Cross JT, Jr., Forciea MA, Hopkins R, Jr., Shekelle P, et al. Current pharmacologic treatment of dementia: a clinical practice guideline from the American College of Physicians and the American Academy of Family Physicians. Annals of internal medicine. 2008;148(5):370-8.

7. Birch D, Draper J. A critical literature review exploring the challenges of delivering effective palliative care to older people with dementia. Journal of clinical nursing. 2008;17(9):1144-63.

8. Kringelbach ML, Jenkinson N, Owen SL, Aziz TZ. Translational principles of deep brain stimulation. Nature reviews Neuroscience. 2007;8(8):623-35.

9. Hamani C, Temel Y. Deep Brain Stimulation for Psychiatric Disease: Contributions and Validity of Animal Models. Sci Transl Med. 2012;4(142):142rv8.

10. Andrade DM, Zumsteg D, Hamani C, Hodaie M, Sarkissian S, Lozano AM, et al. Long-term followup of patients with thalamic deep brain stimulation for epilepsy. Neurology. 2006;66(10):1571-3.

11. Deuschl G, Schade-Brittinger C, Krack P, Volkmann J, Schafer H, Botzel K, et al. A randomized trial of deep-brain stimulation for Parkinson's disease. The New England journal of medicine. 2006;355(9):896-908.

12. Schuurman PR, Bosch DA, Bossuyt PM, Bonsel GJ, van Someren EJ, de Bie RM, et al. A comparison of continuous thalamic stimulation and thalamotomy for suppression of severe tremor. The New England journal of medicine. 2000;342(7):461-8.

13. Bewernick BH, Hurlemann R, Matusch A, Kayser S, Grubert C, Hadrysiewicz B, et al. Nucleus Accumbens Deep Brain Stimulation Decreases Ratings of Depression and Anxiety in TreatmentResistant Depression. Biological psychiatry. 2010;67(2):110-6.

14. Lozano AM, Mayberg HS, Giacobbe P, Hamani C, Craddock RC, Kennedy SH. Subcallosal Cingulate Gyrus Deep Brain Stimulation for Treatment-Resistant Depression. Biological psychiatry. 2008;64(6):461-7.

15. Malone Jr DA, Dougherty DD, Rezai AR, Carpenter LL, Friehs GM, Eskandar EN, et al. Deep Brain Stimulation of the Ventral Capsule/Ventral Striatum for Treatment-Resistant Depression. Biological psychiatry. 2009;65(4):267-75.

16. Denys D, Mantione M, Figee M, van den Munckhof P, Koerselman F, Westenberg H, et al. Deep brain stimulation of the nucleus accumbens for treatment-refractory obsessive-compulsive disorder. Arch Gen Psychiatry. 2010;67(10):1061-8. Epub 2010/10/06. eng. 
17. Hamani C, McAndrews MP, Cohn M, Oh M, Zumsteg D, Shapiro CM, et al. Memory enhancement induced by hypothalamic/fornix deep brain stimulation. Annals of neurology. 2008;63(1):119-23.

18. Laxton AW, Tang-Wai DF, McAndrews MP, Zumsteg D, Wennberg R, Keren R, et al. A phase I trial of deep brain stimulation of memory circuits in Alzheimer's disease. Annals of Neurology. 2010;68(4):521-34.

19. Hescham S, Lim LW, Jahanshahi A, Steinbusch HW, Prickaerts J, Blokland A, et al. Deep brain stimulation of the forniceal area enhances memory functions in experimental dementia: the role of stimulation parameters. Brain Stimul. 2013;6(1):72-7. Epub 2012/03/13. eng.

20. Kuhn J, Hardenacke K, Lenartz D, Gruendler T, Ullsperger M, Bartsch C, et al. Deep brain stimulation of the nucleus basalis of Meynert in Alzheimer's dementia. Molecular psychiatry. 2014.

21. Stone SSD, Teixeira CM, DeVito LM, Zaslavsky K, Josselyn SA, Lozano AM, et al. Stimulation of Entorhinal Cortex Promotes Adult Neurogenesis and Facilitates Spatial Memory. The Journal of Neuroscience. 2011;31(38):13469-84.

22. Suthana N, Haneef Z, Stern J, Mukamel R, Behnke E, Knowlton B, et al. Memory Enhancement and Deep-Brain Stimulation of the Entorhinal Area. New England Journal of Medicine. 2012;366(6):50210.

23. Hescham S, Lim L, Jahanshahi A, Blokland A, Temel Y. Deep brain stimulation in dementia-related disorders. Neurosci Biobehav Rev. 2013;37(10 Pt 2):2666-75. Epub 2013/09/26. eng.

24. Paxinos G, Watson C. The rat brain in steroetaxic coordinates. 1998.

25. Tan S, Vlamings R, Lim L, Sesia T, Janssen ML, Steinbusch HW, et al. Experimental deep brain stimulation in animal models. Neurosurgery. 2010;67(4):1073-9. Epub 2010/10/01. eng.

26. Temel Y, Boothman LJ, Blokland A, Magill PJ, Steinbusch HW, Visser-Vandewalle V, et al. Inhibition of 5-HT neuron activity and induction of depressive-like behavior by high-frequency stimulation of the subthalamic nucleus. Proceedings of the National Academy of Sciences of the United States of America. 2007;104(43):17087-92.

27. Klinkenberg I, Blokland A. The validity of scopolamine as a pharmacological model for cognitive impairment: A review of animal behavioral studies. Neuroscience \& Biobehavioral Reviews. 2010;34(8):1307-50.

28. Cullinan WE, Herman JP, Battaglia DF, Akil H, Watson SJ. Pattern and time course of immediate early gene expression in rat brain following acute stress. Neuroscience. 1995;64(2):477-505.

29. Akkerman S, Prickaerts J, Steinbusch HWM, Blokland A. Object recognition testing: Statistical considerations. Behavioural Brain Research. 2012;232(2):317-22.

30. Sik A, van Nieuwehuyzen P, Prickaerts J, Blokland A. Performance of different mouse strains in an object recognition task. Behavioural Brain Research. 2003;147(1-2):49-54. Epub 2003/12/09. eng.

31. Bondallaz P, Boex C, Rossetti AO, Foletti G, Spinelli L, Vulliemoz S, et al. Electrode location and clinical outcome in hippocampal electrical stimulation for mesial temporal lobe epilepsy. Seizure. 2013;22(5):390-5.

32. Velasco A, Velasco F, Velasco M, Jiménez F, Carrillo-Ruiz JD, Castro G. The role of neuromodulation of the hippocampus in the treatment of intractable complex partial seizures of the temporal lobe. In: Sakas D, Simpson B, editors. Operative Neuromodulation. Acta Neurochirurgica Supplements. 97/2: Springer Vienna; 2007. p. 329-32.

33. Cukiert A, Cukiert CM, Burattini JA, Lima AM. Seizure outcome after hippocampal deep brain stimulation in a prospective cohort of patients with refractory temporal lobe epilepsy. Seizure. 2014;23(1):6-9.

34. McLachlan RS, Pigott S, Tellez-Zenteno JF, Wiebe S, Parrent A. Bilateral hippocampal stimulation for intractable temporal lobe epilepsy: impact on seizures and memory. Epilepsia. 2010;51(2):304-7. 
35. Halgren E, Wilson CL, Stapleton JM. Human medial temporal-lobe stimulation disrupts both formation and retrieval of recent memories. Brain Cogn. 1985;4(3):287-95. Epub 1985/07/01. eng.

36. Lacruz ME, Valentin A, Seoane JJ, Morris RG, Selway RP, Alarcon G. Single pulse electrical stimulation of the hippocampus is sufficient to impair human episodic memory. Neuroscience. 2010;170(2):62332. Epub 2010/07/21. eng.

37. Igarashi KM, Ito HT, Moser EI, Moser M-B. Functional diversity along the transverse axis of hippocampal area CA1. FEBS Letters. 2014;588(15):2470-6.

38. Cassaday HJ, Nelson AJD, Pezze MA. From attention to memory along the dorsal-ventral axis of the medial prefrontal cortex: Some methodological limitations. Frontiers in Systems Neuroscience. 2014;8. English.

39. Weible AP, Rowland DC, Monaghan CK, Wolfgang NT, Kentros CG. Neural Correlates of LongTerm Object Memory in the Mouse Anterior Cingulate Cortex. The Journal of Neuroscience. 2012;32(16):5598-608.

40. Vinogradova OS, Brazhnik ES, Kichigina VF, Stafekhina VS. Modulation of the reaction of hippocampal neurons to sensory stimuli by cholinergic substances. Neuroscience and behavioral physiology. 1996;26(2):113-24.

41. McCartney H, Johnson AD, Weil ZM, Givens B. Theta reset produces optimal conditions for longterm potentiation. Hippocampus. 2004;14(6):684-7.

42. Farovik A, Dupont LM, Eichenbaum H. Distinct roles for dorsal CA3 and CA1 in memory for sequential nonspatial events. Learning \& Memory. 2010;17(1):12-7.

43. Duprez TP, Serieh BA, Raftopoulos C. Absence of Memory Dysfunction after Bilateral Mammillary Body and Mammillothalamic Tract Electrode Implantation: Preliminary Experience in Three Patients. American Journal of Neuroradiology. 2005;26(1):195-8.

44. Hamani C, Dubiela FP, Soares JCK, Shin D, Bittencourt S, Covolan L, et al. Anterior thalamus deep brain stimulation at high current impairs memory in rats. Exp Neurol. 2010;225(1):154-62.

45. Fisher R, Salanova V, Witt T, Worth R, Henry T, Gross R, et al. Electrical stimulation of the anterior nucleus of thalamus for treatment of refractory epilepsy. Epilepsia. 2010;51(5):899-908.

46. Pratt JA. The neuroanatomical basis of anxiety. Pharmacology \& Therapeutics. 1992;55(2):149-81.

47. Catani M, Dell'Acqua F, Thiebaut de Schotten M. A revised limbic system model for memory, emotion and behaviour. Neuroscience \& Biobehavioral Reviews. 2013;37(8):1724-37. 




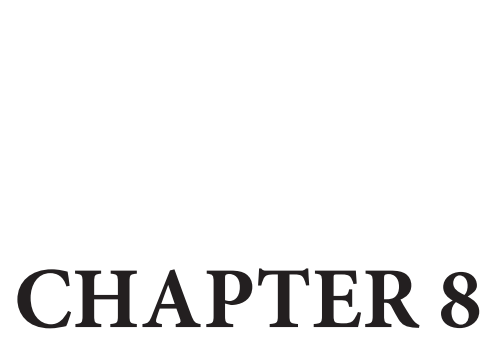

INCREASED NUMBER OF THIMMUNOREACTIVE CELLS IN THE VENTRAL TEGMENTAL AREA AFTER DEEP BRAIN STIMULATION OF THE ANTERIOR NUCLEUS OF THE THALAMUS

Sarah Hescham*, Julie A.D. Dela Cruz ${ }^{*}$, Bryan Adriaanse, Filipa Lopes Campos, Harry W.M. Steinbusch, Bart P.F. Rutten, Yasin Temel, Ali Jahanshahi

${ }^{*}$ Both authors contributed equally

Brain, Structure and Function, 2014; 220(5):3061-6. 


\section{ABSTRACT}

Dopamine (DA) has been long implicated with the processes of memory. In long-term memory, the hippocampus and ventral tegmental area (VTA) use DA to enhance longterm potentiation, while prefrontal DA D1 receptors are involved in working memory. Deep Brain Stimulation (DBS) of specific brain areas have been shown to affect memory impairments in animal models. Here, we tested the hypothesis that DBS could reverse memory impairments by increasing the number of dopaminergic cells in the VTA. Rats received DBS at the level of the mammillothalamic tract, the anterior nucleus of the thalamus, and entorhinal cortex before euthanasia. These regions are part of the so-called memory circuit. Brain sections were processed for c-Fos and tyrosine hydroxylase (TH) immunocytochemistry in the ventral tegmental area (VTA) and the substantia nigra pars compacta (SNc). C-Fos, TH and c-Fos/TH immunoreactive cells were analyzed by means of stereology and confocal microscopy. Our results showed that DBS of the anterior nucleus of the thalamus induced substantial higher numbers of $\mathrm{TH}$-immunoreactive cells in the ventral tegmental area, while there were no significant differences between the experimental groups in the number of $\mathrm{TH}$ immunoreactive cells in the substantia nigra pars compacta, c-Fos immunoreactive cells and c-Fos/TH double-labelled cells in both the substantia nigra pars compacta and ventral tegmental area. Our findings suggest a phenotypic switch, or neurotransmitter respecification, of DAergic cells specifically in the ventral tegmental area which may be induced by DBS in the anterior nucleus of the thalamus. 


\section{INTRODUCTION}

The neurotransmitter dopamine (DA) has been implicated in the process of learning and memory. DA has for example been linked to memory on different topics such as memory studies on sex and reinforcement [1], on drug addiction [2], and feeding preferences [3]. On a cellular level, DA has been shown to positively influence hippocampus dependent learning and memory by enhancing long-term potentiation (LTP) in the hippocampus through D1/D5 receptors (mainly the CA1 region) [4] by interacting with the ventral tegmental area (VTA). The hippocampus is also part of the circuit of Papez, which has been linked to episodic and spatial memory [5].

Memory impairments are the core features of the different types of dementia and researchers have been exploring the application of non-pharmacological therapies in order to reduce or delay the progression of dementia. One treatment currently being tested is deep brain stimulation (DBS). Findings from recent preclinical studies [6, 7] and clinical studies [8] have suggested that DBS can be used as a tool to enhance memory functions, including reversing memory impairments.

In addition to restoring memory loss, DBS is also used to treat other disorders, such as Parkinson's disease, Tourette's syndrome and several forms of epilepsy [9]. DBS has been proposed as an alternative to drug therapy and has the advantages of reversibility and adjustability in patients [10]. Changes in DA levels through DBS may have some impact in other types of disorders.

In the present study, DBS was implemented in different brain regions of rats with scopolamine-induced memory impairments. The current study focuses on the quantifications of the number of tyrosine hydroxylase $(\mathrm{TH}$, a rate-limiting enzyme in the formation of DA) immunoreactive cells, the number of c-Fos (a proto-oncogene and marker for neuronal activation) immunoreactive cells and the co-localization of the two markers in the major sites of DA synthesis: the Ventral Tegmental Area (VTA) and the Substantia Nigra pars compacta (SNc). It is hypothesized that DBS in various stimulation sites [circuit of Papez structures: Entorhinal Cortex (EC), Mammillothalamic Tract (MTT) and the Anterior Nucleus of the Thalamus (ANT)] will have differential effects on the number of TH-immunoreactive cells or co-localized TH/c-Fos immunoreactive cells in the VTA and SNc after DBS and scopolamine-induced memory impairments.

\section{MATERIALS AND METHODS}

This is a summarized section. For a complete "Material and Methods" section, please see the online resource for this article. 
Experimental groups

Sprague Dawley (SD) rats were assigned at random to receive DBS in one of several brain sites: the mammillothalamic tract (MTT, $n=5)$, the entorhinal cortex $(E C, n=6)$, the anterior nucleus of the thalamus (ANT, n=5) and a sham-stimulated group, without scopolamine injections $(n=3)$ was made for a negative control.

Pre-perfusion experimental procedures

Scopolamine-model of memory impairments was used during a previous behavioral study. Before each behavioral task rats were injected with scopolamine and stimulated with various parameters during testing. For more information please see [7].

\section{Deep brain stimulation}

Before prefusion took place, three different experimental conditions were given: (a) DBS at an amplitude of $100 \mu \mathrm{A}$ in the EC (b) DBS at an amplitude of $50 \mu \mathrm{A}$ in the ANT and (c) an amplitude of $200 \mu \mathrm{A}$ in the MTT. The used stimulation frequency was $100 \mathrm{~Hz}$ and the pulse width was set at $100 \mu$ s. All animals were stimulated for $1 \mathrm{~h}$ and allowed to rest for $1 \mathrm{~h}$ before sacrifice.

\section{Immunohistochemistry}

After perfusion, tissue was collected and cut into $30 \mu \mathrm{m}$ slices on a vibratome. A fluorescence protocol was conducted for the visualization of TH and c-Fos. First, primary antibodies of mouse anti-TH (1:100, Médimabs, Montreal, Canada) and rabbit anti- c-Fos (1:500, Santa Cruz, Santa Cruz California, USA) were incubated overnight. The next day, the slices had incubation of the secondary antibodies: Alexa 594 donkey anti-mouse (1:100, Invitrogen, Grand Island, NY, USA) followed by Alexa 488 Donkey anti-rabbit (1:100, Invitrogen, Grand Island, NY) for $2 \mathrm{~h}$ each.

\section{Histological analysis and quantification}

For analysis and cell counting of $\mathrm{TH}$ immunoreactive, c-Fos immunoreactive and c-Fos/TH double-labeled cells in the SNc and VTA of the brain sections, a spinning disk confocal microscope (Olympus DSU BX51WI, Pennsylvania, USA) was used. In all sections showing the SNc and VTA were delineated and the total numbers of TH containing cells were estimated with the optical fractionator. For more details about the stereologic counting procedure see [11]. On the settings and data of our current quantifications, see the online resource.

\section{Statistical analysis}

One-way ANOVA's were performed using SPSS (version 18 for Apple, SPSS, Chicago, IL, USA), and post-hoc multiple comparisons test were performed with a Tukey's Hon- 
est Significant Difference (HSD) test, due to the fact that it has the best all-possible pairwise comparisons when sample sizes are unequal. A p-value $<0.05$ was considered to be significant.

\section{RESULTS}

\section{Number of TH-immunoreactive cells in the VTA and SNc}

Through qualitative visual inspection, it would be difficult to discern the difference in TH-levels just by looking at the brain sections (Figure 1), therefore stereology was conducted to analyze the data quantitatively. The one-way ANOVA indicated that DBS had a significant effect on the number of TH-immunoreactive cells in the VTA $(F(3,15)=5.902, p<0.05$; Figure 2). Post hoc comparisons using the Tukey's HSD indicated that the mean number of TH-immunoreactive cells was significantly higher for the ANT-stimulated group $(M=24,773, \mathrm{SEM}=942)$ as compared with the MTT-stimulated group $(M=18,884, S E M=1,586)$, the EC-stimulated group $(M=20,694, S E M=840)$, and the sham-stimulated group $(M=16,648, S E M=2,474)$ (Figure 2A). No significant difference was found between the MTT-stimulated group, the EC-stimulated group and the sham-stimulated group. In addition, TH cell measurements in the VTA and VTA volume measurements were not significantly different throughout the four conditions. Lastly, no significant differences were found for the number of TH-immunoreactive cells in the SNc (Figure 2B).

\section{c-Fos immunoreactive cells and double-labelled c-Fos/TH cells in the VTA and the $\mathrm{SNc}$}

One-way ANOVAs were conducted to compare the effect of c-Fos immunoreactive cells in both the VTA and the SNc of different DBS target structures. There were no significant differences found on c-Fos immunoreactive cells in the VTA, the SNc, or double-labeled c-Fos/TH cells between groups at the $\mathrm{p}<0.05$ level (Figure 3 ).

\section{DISCUSSION}

ANT-DBS increased the number of TH-immunoreactive cells in the VTA when compared to the EC-stimulated, MTT-stimulated and sham group, while no difference was found in the SNc. The c-Fos immunoreactive cells and the double-labelled c-Fos/ $\mathrm{TH}$ cells were not found to be significantly different between the conditions. From the c-Fos and double-labeled results, it can be concluded that DA, or any other active 

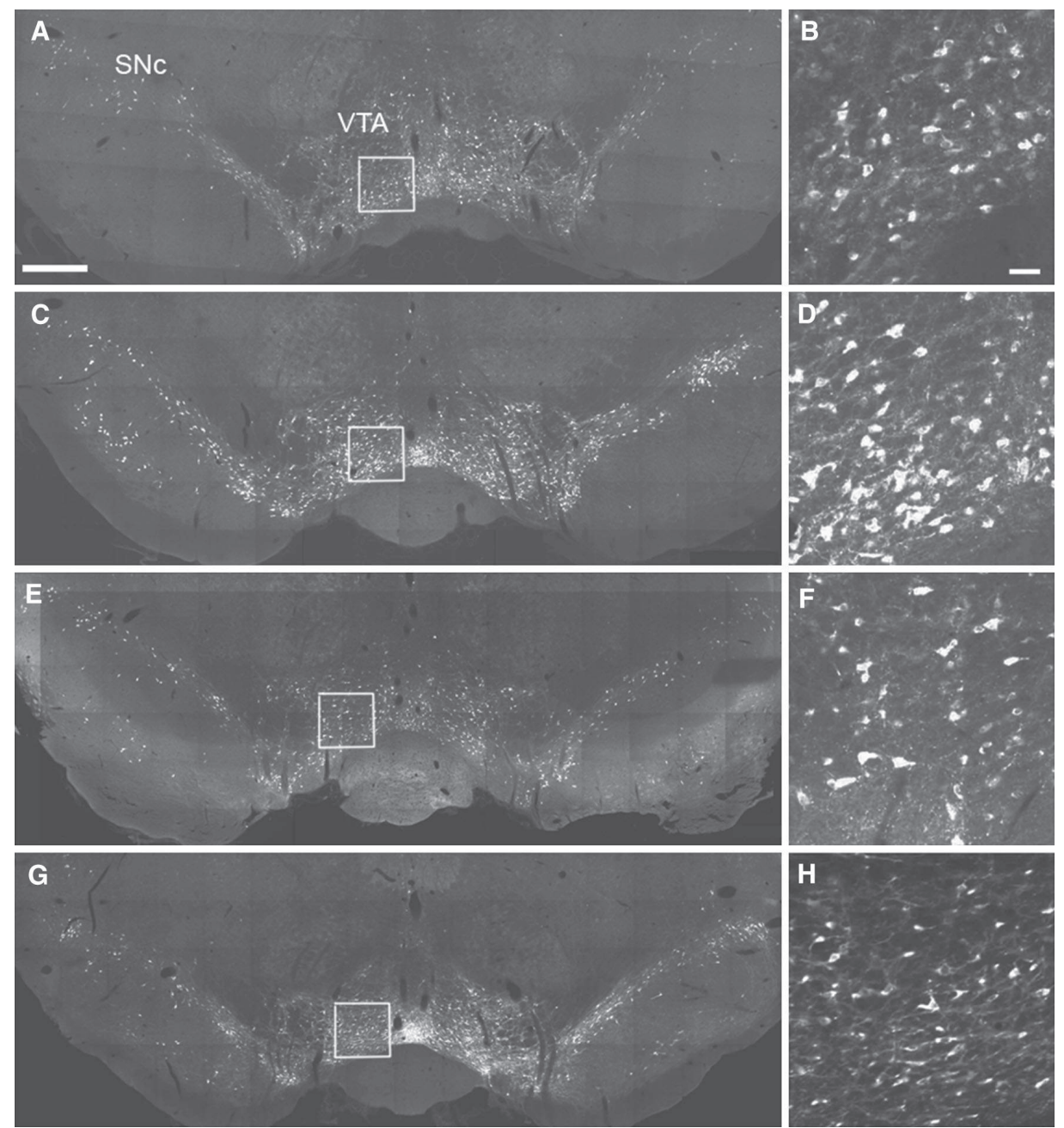

Figure 1: All pictures are around bregma level $-6.00 \mathrm{~mm}$. A) Overview of TH immunoreactive cells in the VTA and SNc for a MTT stimulated rat. B) The VTA in a MTT stimulated rat. C) Overview for an ANT stimulated rat. D) The VTA in an ANT stimulated rat. E) Overview for an EC stimulated rat. F) The VTA in an EC stimulated rat. G) Overview for a sham rat. H) The VTA in a sham rat. Scale bars, A), C), E) and G) $500 \mu \mathrm{m}$; B), D), F), H) $50 \mu \mathrm{m}$.

neurons in the VTA or SNc, were not involved in the activation of processes by DBS before being sacrificed.

One theory to explain the higher levels of TH is phenotypic switching, or neurotransmitter respecification. In a recent study, some populations of interneurons found in the adult rat hypothalamus switched between dopamine and somatostatin in response to exposure to short- and long-day photoperiods [12]. Furthermore, a following study found changes in TH levels in the SNc and VTA when exposed to differing manipula- 

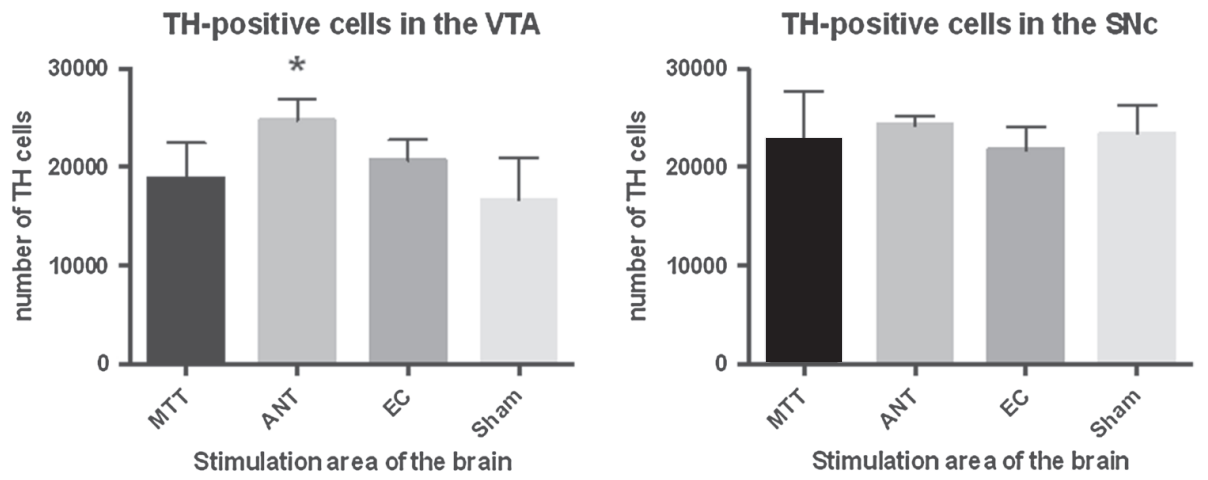

Figure 2: Number of TH-immunoreactive cells in the VTA and the SNc after DBS. A) The group that was stimulated in the Anterior Nucleus of the Thalamus (ANT, $n=5$ ) was found to have significantly more TH-immunoreactive cells in the VTA than the groups stimulated in the Mammillothalamic Tract (MTT, $n=5)$, in the Entorhinal Cortex (EC, $n=6)$, and the sham stimulated group $\left.(n=3) .{ }^{\star} p<0.05, B\right)$ there was no significant difference found between the four groups in the SNc.
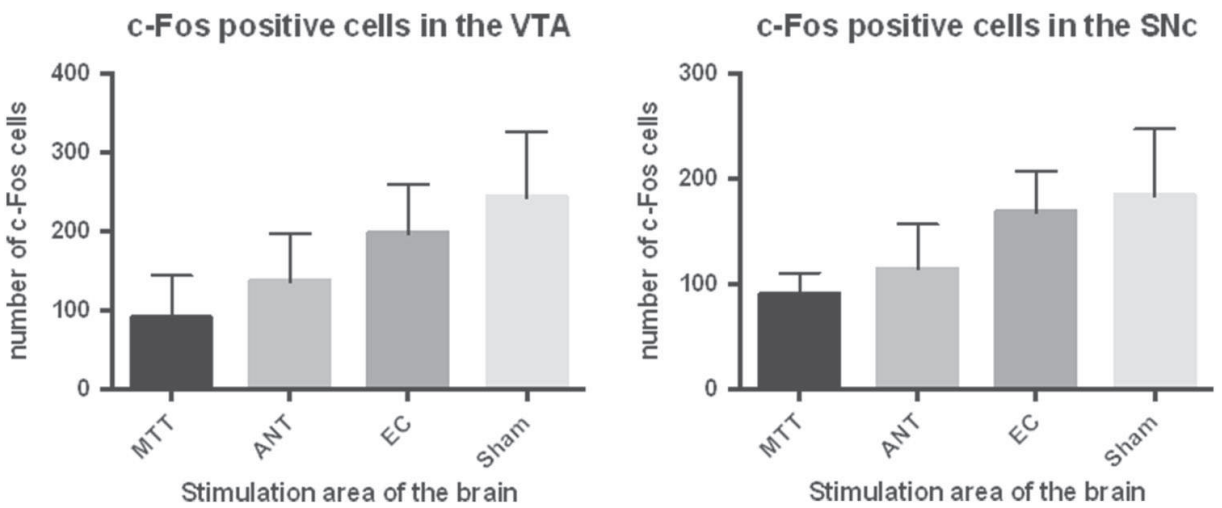

Figure 3: The number of c-fos cells in the A) VTA and B) SNc after DBS. No significant difference was found in the c-Fos positive levels between the groups stimulated in the Mammilothalamic Tract (MTT, $n=5$ ), in the Anterior Nucleus of the Thalamus (ANT, $n=5)$, in the Entorhinal Cortex (EC, $n=6$ ), and the sham stimulated group $(\mathrm{n}=3)$.

tions of environmental stimuli $[13,14]$. More TH-immunoreactive cells were found in environmentally enriched males, while this increase was lost after an infusion of a GABA receptor antagonist [14]. Recently, in postmortem human Huntington's disease (HD) tissue and in the transgenic rat model of HD, we found an increased number of dopaminergic neurons, which was accompanied by a decreased number of serotonergic neurons in the dorsal raphe nucleus [15], suggesting that a pathological condition such as genetic mutation can lead to neurotransmitter respecification as well. These studies 
show that neurons, in the brain are not fixed, but respond to environmental stimuli and behaviors.

Dopaminergic neurons comprise 50-60\% of all neurons in the VTA [16], while GABAergic neurons comprise a sizable population [17]. Presumably electrical stimulation in the ANT, and not the MTT or the EC, causes shifts in transcriptional level from one type of neurotransmitter cell (i.e. GABAergic cells) to a DA cell in an attempt to have non-invasive restoration of the normal functioning nervous system. One suggestion for a subsequent study would be to double-stain TH with GAD67, an antibody that tags GABA cell bodies to examine the neuronal compositions of the VTA between the four conditions of this study.

An alternative interpretation for the increase in TH cells in the VTA can be explained through neurogenesis. Stimulating the ANT might have modulated TH cell migration to the VTA. Due to the lack of TH/c-Fos colocalization, it can be speculated that these cells have not been functionally integrated at the time of sacrifice. DBS of specific target regions has been shown to cause neurogenesis. DBS of the EC [6] and ANT [18] for example, have shown to induce hippocampal neurogenesis in neurogenic regions, e.g. hippocampus. While brainstem neurogenesis has been reported earlier [19] later studies did not confirm those findings.

Interestingly, electrical stimulation of the ANT was found effective for treating refractory focal epilepsy in a clinical trial [20], while memory problems were reported as adverse effects [21], which may be in part due to its central connectivity [22]. In line with the finding from these studies, memory improvement was observed in rats stimulated in the EC during behavioral studies [6], while memory impairments were retained in rats stimulated in the ANT [18]. DBS effects in the MTT and memory are still unknown.

In conclusion, our findings suggest a phenotypic switch between GABA neurons and DA neurons in the VTA following ANT-DBS. The environmental stimuli of DBS in the ANT, but not DBS in the MTT or EC, may influence this increase in THimmunoreactive cells in the VTA. As dopaminergic projections from the VTA mediate reward-seeking behavior (among other functions), our current findings may imply that ANT-stimulation with DBS, such as used in the treatment of epilepsy, may induce alterations in reward-seeking and other behavioral phenotypes.

\section{ACKNOWLEDGEMENTS}

The micrographs in this paper were taken with a confocal spinning disk microscope financed by The Netherlands Organisation for Scientific Research (NWO), grant number 911-06-003. 


\section{REFERENCES}

1. Becker JB, Rudick CN, Jenkins WJ. The role of dopamine in the nucleus accumbens and striatum during sexual behavior in the female rat. The Journal of neuroscience : the official journal of the Society for Neuroscience. 2001;21(9):3236-41.

2. Carr KD. Chronic food restriction: enhancing effects on drug reward and striatal cell signaling. Physiology \& behavior. 2007;91(5):459-72.

3. Dela Cruz JA, Icaza-Cukali D, Tayabali H, Sampson C, Galanopoulos V, Bamshad D, et al. Roles of dopamine D1 and D2 receptors in the acquisition and expression of fat-conditioned flavor preferences in rats. Neurobiology of learning and memory. 2012;97(3):332-7.

4. Lisman JE, Grace AA. The hippocampal-VTA loop: controlling the entry of information into longterm memory. Neuron. 2005;46(5):703-13.

5. Hescham S, Lim L, Jahanshahi A, Blokland A, Temel Y. Deep brain stimulation in dementia-related disorders. Neurosci Biobehav Rev. 2013;37(10 Pt 2):2666-75. Epub 2013/09/26. eng.

6. Stone SSD, Teixeira CM, DeVito LM, Zaslavsky K, Josselyn SA, Lozano AM, et al. Stimulation of Entorhinal Cortex Promotes Adult Neurogenesis and Facilitates Spatial Memory. The Journal of Neuroscience. 2011;31(38):13469-84.

7. Hescham S, Lim LW, Jahanshahi A, Steinbusch HW, Prickaerts J, Blokland A, et al. Deep brain stimulation of the forniceal area enhances memory functions in experimental dementia: the role of stimulation parameters. Brain Stimul. 2013;6(1):72-7. Epub 2012/03/13. eng.

8. Laxton AW, Tang-Wai DF, McAndrews MP, Zumsteg D, Wennberg R, Keren R, et al. A phase I trial of deep brain stimulation of memory circuits in Alzheimer's disease. Annals of Neurology. 2010;68(4):521-34.

9. Wichmann T, Delong MR. Deep brain stimulation for neurologic and neuropsychiatric disorders. Neuron. 2006;52(1):197-204.

10. Theodore WH, Fisher RS. Brain stimulation for epilepsy. The Lancet Neurology. 2004;3(2):111-8.

11. Temel Y, Visser-Vandewalle V, Kaplan S, Kozan R, Daemen MA, Blokland A, et al. Protection of nigral cell death by bilateral subthalamic nucleus stimulation. Brain Res 2006;1120(1):100-5.

12. Dulcis D, Jamshidi P, Leutgeb S, Spitzer NC. Neurotransmitter switching in the adult brain regulates behavior. Science. 2013;340(6131):449-53.

13. Aumann TD, Egan K, Lim J, Boon WC, Bye CR, Chua HK, et al. Neuronal activity regulates expression of tyrosine hydroxylase in adult mouse substantia nigra pars compacta neurons. Journal of neurochemistry. 2011;116(4):646-58.

14. Aumann TD, Tomas D, Horne MK. Environmental and behavioral modulation of the number of substantia nigra dopamine neurons in adult mice. Brain and Behavior. 2013;3(6):617-25.

15. Jahanshahi A, Vlamings R, van Roon-Mom WM, Faull RL, Waldvogel HJ, Janssen ML, et al. Changes in brainstem serotonergic and dopaminergic cell populations in experimental and clinical Huntington's disease. Neuroscience. 2013;238:71-81.

16. Margolis EB, Lock H, Hjelmstad GO, Fields HL. The ventral tegmental area revisited: is there an electrophysiological marker for dopaminergic neurons? The Journal of Physiology. 2006;577(3):90724.

17. Oades RD, Halliday GM. Ventral tegmental (A10) system: neurobiology. 1. Anatomy and connectivity. Brain Res. 1987;434(2):117-65.

18. Hamani C, Stone SS, Garten A, Lozano AM, Winocur G. Memory rescue and enhanced neurogenesis following electrical stimulation of the anterior thalamus in rats treated with corticosterone. Exp Neurol. 2011;232(1):100-4. Epub 2011/09/13. eng. 
19. Zhao M, Momma S, Delfani K, Carlen M, Cassidy RM, Johansson CB, et al. Evidence for neurogenesis in the adult mammalian substantia nigra. Proc Natl Acad Sci U S A. 2003;100(13):7925-30.

20. Graber KD, Fisher RS. Deep Brain Stimulation for Epilepsy: Animal Models. In: Noebels JL, Avoli M, Rogawski MA, Olsen RW, Delgado-Escueta AV, editors. Jasper's Basic Mechanisms of the Epilepsies. 4th ed. Bethesda (MD)2012.

21. Fisher R, Salanova V, Witt T, Worth R, Henry T, Gross R, et al. Electrical stimulation of the anterior nucleus of thalamus for treatment of refractory epilepsy. Epilepsia. 2010;51(5):899-908.

22. Child ND, Benarroch EE. Anterior nucleus of the thalamus: Functional organization and clinical implications. Neurology. 2013;81(21):1869-76. 




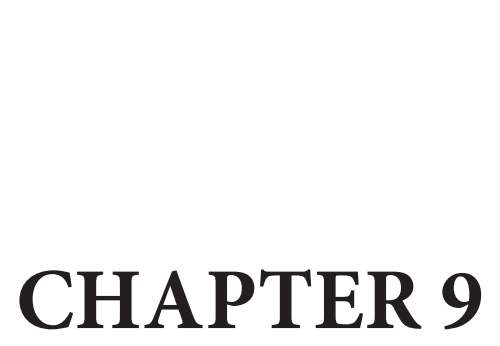

FORNIX DEEP BRAIN STIMULATION ENHANCES ACETYLCHOLINE LEVELS IN THE HIPPOCAMPUS

Sarah Hescham, Ali Jahanshahi, Judith V Schweimer, Stephen N Mitchell, Guy Carter, Arjan Blokland, Trevor Sharp and Yasin Temel

Brain, Structure and Function, 2015 (in press). 


\begin{abstract}
Deep brain stimulation (DBS) of the fornix has gained interest as a potential therapy for advanced treatment-resistant dementia, yet the mechanism of action remains widely unknown. Previously we have reported beneficial memory effects of fornix DBS in a scopolamine-induced, rat model of dementia, which is dependent on various brain structures including hippocampus. To elucidate mechanisms of action of fornix DBS with regard to memory restoration, we performed c-Fos immunohistochemistry in the hippocampus. We found that fornix DBS induced a selective activation of cells in the CA1 and CA3 subfields of the dorsal hippocampus. In addition, hippocampal neurotransmitter levels were measured using microdialysis before, during and after $60 \mathrm{~min}$ of fornix DBS in a next experiment. We observed a substantial increase in the levels of extracellular hippocampal acetylcholine, which peaked $20 \mathrm{~min}$ after stimulus onset. Interestingly, hippocampal glutamate levels did not change compared to baseline. Therefore, our findings provide first experimental evidence that fornix DBS activates the hippocampus and induces the release of acetylcholine in this region.
\end{abstract}




\section{INTRODUCTION}

Dementia is a major threat to human population. The World Health Organization estimates that 35.6 million people suffer from dementia, a number that is anticipated to triple by 2050 [1]. The most common form of dementia is Alzheimer's disease (AD), accounting for $60-80 \%$ of all cases. So far there are no known treatments available that cure or delay the progression of $\mathrm{AD}$. Current pharmacological treatments are not effective for every patient and only alleviate symptoms temporarily [2, 3].

Recently, the application of DBS to reduce or delay the progression of memory loss in $\mathrm{AD}$ has shown to be effective. In particular, the convergence of the latest observations in humans and rats pinpoints that DBS of the fornix can restore memory loss [4-6]. In our own experiments [5], fornix DBS reduced memory deficits induced by the muscarinic receptor antagonist scopolamine in a rat model. In this scopolamine-induced rat model of memory impairment various brain structures including the hippocampus are affected [7]. The mechanism of action of fornix DBS is not well understood but the latter suggests an involvement of the hippocampus and acetylcholine.

In the present study, we therefore investigated the effects of fornix DBS on the hippocampus with a dual approach. Firstly, we evaluated c-Fos expression in the dorsal hippocampus. The immediate early gene c-Fos is an indirect marker of neuronal activation and can be even used to map long-term activation $[8,9]$. Secondly, we investigated the effect of fornix DBS on levels of hippocampal acetylcholine as well as glutamate; neurotransmitters which are widely acknowledged as important for memory functions $[10,11]$.

\section{MATERIALS AND METHODS}

\section{Experimental design}

This study consists of two experiments:

Experiment 1: Male Sprague-Dawley rats (Charles River, Sulzfeld, Germany) were either assigned to the fornix DBS $(n=5)$ or sham $(n=5)$ group. Electrode implantations were performed as described previously [5]. DBS electrodes were implanted at the site of the fornix (coordinates from bregma according to the rat brain atlas of Paxinos and Watson [12]: AP: $-1.8 \mathrm{~mm}$; ML: $1.3 \mathrm{~mm}$; DV: $-8.0 \mathrm{~mm}$ ). It was found that fornix DBS reduced memory deficits induced by the muscarinic receptor antagonist scopolamine in a hippocampal-dependent rat model as reported elsewhere [5]. In these same animals, one week later (when scopolamine is no longer present), rats were stimulated at $100 \mathrm{~Hz}, 100 \mu \mathrm{A}$ and $100 \mu$ s pulse width for $1 \mathrm{~h}$ and allowed to rest for $1 \mathrm{~h}$. 
Sham rats were only attached to cables, but not stimulated. Subsequently, animals were transcardially perfused, first with Tyrode's solution and then Somogyi fixative [13]. Animal procedures in experiment 1 were approved and carried out in accordance to the Animal Experiments and Ethics Committee of Maastricht University.

Experiment 2: In order to establish whether fornix DBS effects the neurochemistry in the hippocampus, microdialysis experiments were carried out in male Sprague-Dawley rats (Harlan, Bicester, U.K., fornix DBS $n=13$ and sham $n=6$ ) under urethane anaesthesia. Animal procedures in experiment 2 were carried out in accordance with the UK Animals (Scientific Procedures) Act 1986 and associated Home Office guidelines, and with local ethical approval.

\section{Immunohistochemistry}

Following transcardial perfusion in experiment 1 , brains were collected and cut into $30 \mu \mathrm{m}$ slices on a vibratome (Leica ${ }^{\star}$, Wetzlar, Germany). For immunohistochemistry, sections were incubated overnight with polyclonal rabbit anti-c-Fos primary antibody (1:2000; K-25, Santa Cruz Biotechnology Inc, Santa Cruz, USA) followed by biotinylated donkey anti-rabbit secondary antibody (1:800; Jackson Immunoresearch Laboratories Inc., Westgrove, USA) and avidin-biotin peroxidase complex (1:800, Elite ABC-kit, Vectastain ${ }^{\oplus}$, Burlingame, CA, USA). The staining was visualized by 3,3'-Diaminobenzidine $(\mathrm{DAB})$ combined with $\mathrm{NiCl}_{2}$ intensification.

Images were taken at two similar bregma levels were taken using a U-CMAD-2 digital camera connected to an Olympus AX70 brightfield microscope (analySIS; Imaging System, Münster, Germany), and evaluated with ImageJ (Image J software version 1.38x; NIH, Bethesda, USA). C-Fos-positive cells in the medial prefrontal cortex and hippocampus were counted manually by an observer blind to treatment.

\section{In-vivo microdialysis}

For experiment 2, rats were anaesthetized with 25\% urethane (Ethyl carbamate, Sigma) and mounted in a stereotaxic frame. DBS electrodes were implanted at the site of the fornix, and a single cannula microdialysis probe (CMA11, tip length $2 \mathrm{~mm}$, CMA Microdialysis, Kista, Sweden) was implanted into the hippocampus (coordinates from bregma: AP: $-4.8 \mathrm{~mm}$; ML: $3 \mathrm{~mm}$; DV: $-4.2 \mathrm{~mm}$ ). Microdialysis probes were perfused with artificial cerebrospinal fluid (141 mM NaCl, $5 \mathrm{mM} \mathrm{KCl}, 0.8 \mathrm{mM} \mathrm{MgCl}, 1.5 \mathrm{mM}$ $\mathrm{CaCl}_{2}$ ) at a flow rate of $1.5 \mu \mathrm{l} / \mathrm{min}(\mathrm{CMA} / 100$, Carnegie Medicine) for $2 \mathrm{~h}$ before dialysate collection started. Dialysate samples were collected every $20 \mathrm{~min}$, DBS stimulation time $(100 \mathrm{~Hz}, 100 \mu \mathrm{A}$ and $100 \mu \mathrm{s})$ was $1 \mathrm{~h}$. In total 10 samples were collected (4 baseline, 3 during stimulation and 3 after stimulation). Samples were immediately frozen on dry ice and later analyzed with liquid chromatography/mass spectrometry. 


\section{Liquid chromatography/mass spectrometry}

All microdialysis samples were analyzed at Eli Lilly Research Centre, Windlesham, U.K. The high-performance liquid chromatography (HPLC) system consisted of a PAL HTC-xt autosampler (CTC Analytics AG Zwingen, Switzerland), a pair of Shimadzu LC-20AD XR pumps with a Shimadzu CBM-20A controller and Shimadzu CTO-20AD column oven (Shimadzu Ltd, Milton Keynes, U.K.). Injection volume was $10 \mu$. The chromatographic retention was obtained by using an XBridge BEH Amide column (75 x $2.1 \mathrm{~mm}$, i.d. $2.5 \mu \mathrm{m}$; Waters Ltd, Elstree, U.K.) at $35^{\circ} \mathrm{C}$. The gradient elution was carried out using acetonitrile and $2 \mathrm{mM}$ ammonium formate, 95:5 and 5:95 (the $\mathrm{pH}$ of 3.0 was adjusted with formic acid). Detection was carried out by an AB Sciex Triple Quad API5500 mass spectrometer MS (AB Sciex UK Ltd, Warrington, U.K.), which operated in TurbolonSpray ${ }^{\circledast}$ mode using a Turbo $\mathrm{V}^{\mathrm{max}}$ source and SRM analysis.

Acetylcholine was detected by monitoring the $\mathrm{m} / \mathrm{z} 146.1 \rightarrow 87.0$ transition and its D4 analogue internal standard at $\mathrm{m} / \mathrm{z} 150.1 \rightarrow 91.0$ (dwell time: $50 \mathrm{~ms}$, collision energy: $20 \mathrm{~V}$, collision cell exit potential: $4.2 \mathrm{~V}$ ). Glutamic acid was detected by monitoring the $\mathrm{m} / \mathrm{z} 148.0 \rightarrow 84.0$ transition and its D5 analogue internal standard at $\mathrm{m} / \mathrm{z} 153.0$ $\rightarrow 88.0$ (dwell time: $50 \mathrm{~ms}$, collision energy: $23 \mathrm{~V}$, collision cell exit potential: $10 \mathrm{~V}$ ). Samples were prepared by 1:10 dilution in internal standard and a single acetylcholine calibration curve between 0.10 and $20.0 \mathrm{nM}$ and glutamic acid curve between 50 and $2000 \mathrm{nM}$ was run at the end of each batch of samples for quantification.

\section{Verification of DBS electrodes and microdialysis probes}

Sections containing the electrode trajectories from all animals were collected and mounted on gelatin coated glass slides. A standard haematoxylin-eosin staining was carried out before sections were photographed under bright field microscopy.

\section{Statistical analysis}

For experiment 1, the number of Fos-positive cells was expressed as percentage of change when compared to sham (i.e. the average of Fos-positive cells in sham was chosen as reference value and defined as 100\%). Raw values as well as percentage scores were normally distributed as assessed by the Shapiro Wilk's test. For better comparison between the groups, however, we chose to present the percentage scores.

An independent-samples t-test was employed and $\mathrm{p}$-values $<0.05$ were considered significant. Microdialysis data of experiment 2 were represented as percentage of the mean of 4 baseline samples prior to DBS. The effect of fornix DBS on extracellular acetylcholine and glutamate levels over time was analyzed by repeated-measures ANOVA followed by Fisher's least significant difference (LSD) post-hoc test. 


\section{RESULTS}

\section{Verification of DBS electrodes and microdialysis probes}

The location of all DBS electrodes was verified within the fornix, and microdialysis probes were all correctly placed in the dorsal hippocampus. For the current stimulation settings used, we found no evidence for histological damage as observed by a standard haematoxylin-eosin staining.

\section{Immunohistochemistry}

We found increased c-Fos expression in the CA1 subregion for fornix stimulated animals when compared to sham $(\mathrm{t}(8)=4.285 ; \mathrm{p}<0.01)$. In addition, increased levels of c-Fos cells in the CA3 subregion were observed in fornix DBS rats $(\mathrm{t}(8)=4.363$; $\mathrm{p}<0.01$ ). There was no statistical difference between levels of $\mathrm{c}$-Fos expression in the dentate gyrus of fornix stimulated and sham rats $(t(7)=0.455$; n.s.; Fig. 1$)$.
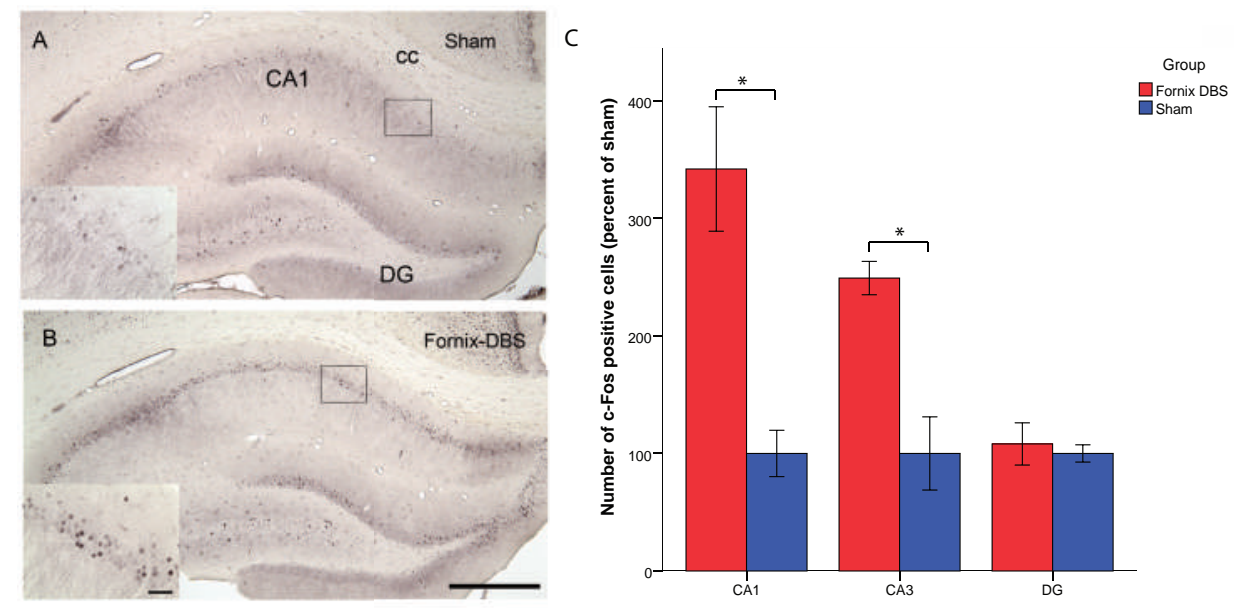

Figure 1: Representative low-power photomicrographs (scale bar $=500 \mu \mathrm{m})$ of coronal brain sections stained for c-Fos (K-25) showing the hippocampus of sham (A) and fornix stimulated animals (B). The high-power photomicrograph insets in the lower left corner show the CA1 subregion of the hippocampus (scale bar $=50 \mu \mathrm{m})$. C) Comparisons were made as percentage increase/decrease with regard to sham. Fornix DBS rats show increased c-Fos (K-25) expression in the CA1 and CA3 subregion of the hippocampus when compared to sham. There was no statistical difference between sham and DBS groups in the dentate gyrus. ${ }^{*} \mathrm{p}<0.05$, independent samples $\mathrm{t}$-test for fornix DBS vs. sham rats. Data represents mean \pm S.E.M. DG, dentate gyrus; cc, corpus callosum. 


\section{In-vivo microdialysis}

Fornix DBS for $60 \mathrm{~min}$ caused a significant increase in hippocampal acetylcholine levels in comparison to non-stimulated controls (repeated measures ANOVA: $\mathrm{F}(1,17)=6,608 ; \mathrm{p}<0.03$ ). This effect was evident in the first $20 \mathrm{~min}$ of fornix DBS as shown by the LSD post-hoc test ( $\mathrm{p}<0.03$; Fig. $2 \mathrm{~A}$ ). Acetylcholine levels in other time points during stimulation as well as after stimulation did not differ between the groups. There was no statistical difference for hippocampal glutamate levels between fornix DBS and sham (repeated measures ANOVA: F $(1,16)=0.038$; n.s.; Fig. 2B).

\section{DISCUSSION}

According to several studies, fornix DBS in AD patients leads to a decreased rate of cognitive decline $[6,14]$; yet, a potential mechanism of action has not been found. In the present study, we found that beneficial memory effects of fornix DBS might be linked to an enhanced neural activity in the CA1 and CA3 subfield, suggesting increased activity of certain neurons in this region. The CA1 and CA3 subregions of the hippocampus are important for spatial memory $[15,16]$. In particular, the CA3 subregion plays a role in encoding and retrieval of spatial location sequences and the CA1 contributes to memory encoding by binding cues to their temporal context, which in turn also enables retrieval of location sequences [15]. Interestingly, a substantial neuronal loss in the CA1 is observed in postmortem AD brains [17]. If fornix DBS is able to increase neural activity in the CA1 subfield, then it may compensate for reduced neuronal integrity of this region in $\mathrm{AD}$.

In the etiology of $\mathrm{AD}$, evidence exists for both cholinergic and glutamatergic involvement [18]. In the present study, microdialysis data revealed that hippocampal glutamate levels were not affected by fornix DBS. A clear connection between glutamate and memory-related processes is provided by the association of ionotropic glutamate receptors in the induction of long-term potentiation. Although memory can be disrupted by blockade of these receptors (e.g. N-methyl-D-aspartate, NMDA) [19], the enhancement of the glutamatergic signal may also have adverse effects on memory because high levels of glutamate are neurotoxic [20]. Our results suggest that fornix DBS does not influence glutamate-dependent long-term potentiation, but also does not induce excitotoxicity.

Contrary to this, fornix DBS did have an effect on extracellular hippocampal acetylcholine and it might be possible that increased c-Fos expression in the hippocampus was mediated by enhanced acetylcholine levels [21]. In an earlier study, we have shown that fornix DBS was able to restore scopolamine-induced memory deficits when stimulating 


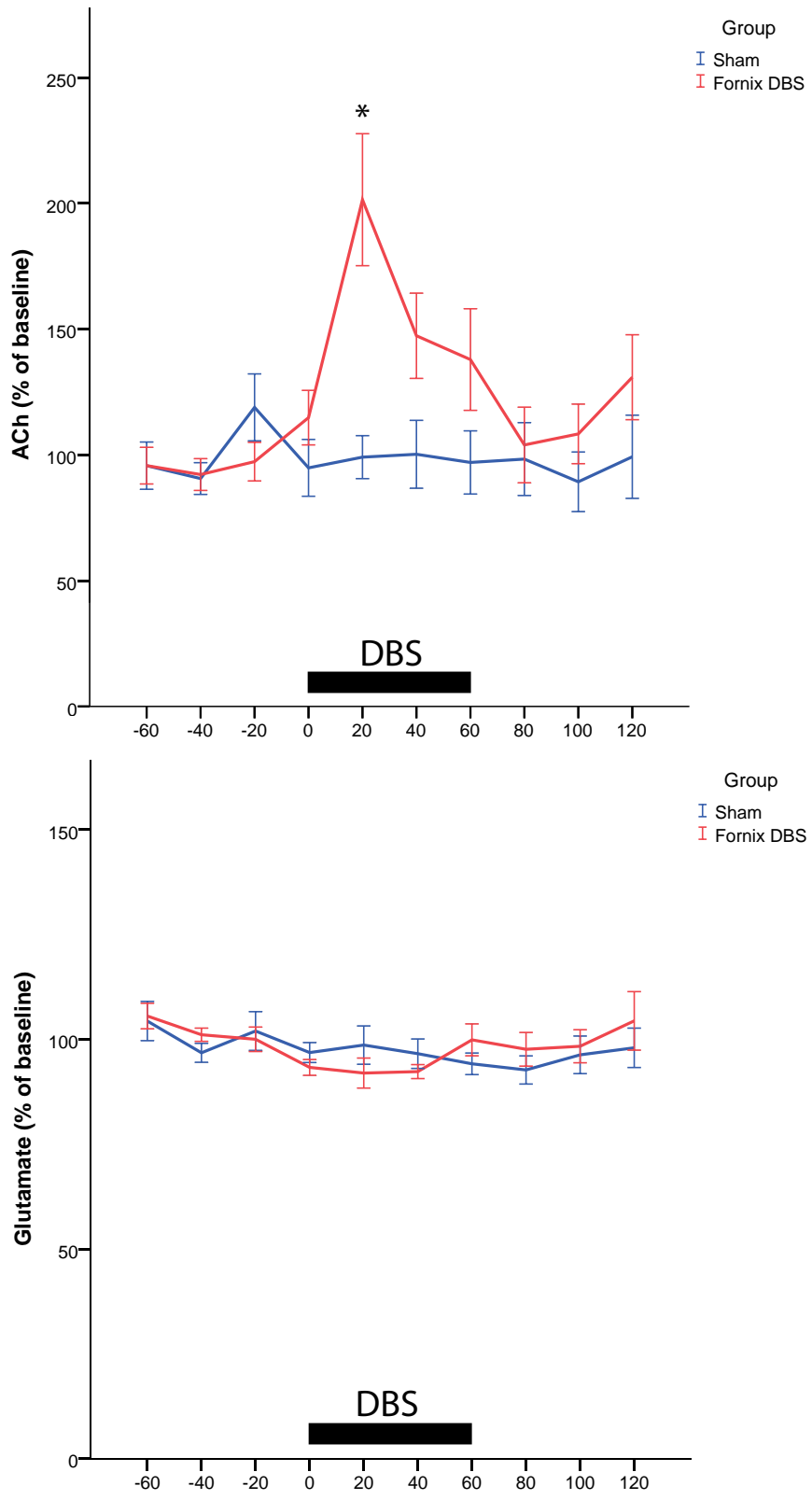

Figure 2: Microdialysate acetylcholine (Ach, A) and glutamate (B) levels of the dorsal hippocampus in anesthetized fornix DBS $(n=13)$ and sham rats $(n=6)$. Horizontal bar indicates stimulation period. Acetylcholine levels were significantly elevated in the fornix DBS group after 20 min of stimulation. No difference in glutamate levels was detected between fornix DBS and sham. Data points are mean \pm S.E.M. values expressed as a $\%$ of baseline. ${ }^{*} \mathrm{p}<0.05$, repeated measures ANOVA for fornix DBS vs. sham rats. 
the fornix for 5 min during the acquisition and retention trial of the object location task, a test of hippocampal-dependent memory [5, 22]. Potentially, the increased hippocampal acetylcholine measured via microdialysis after $20 \mathrm{~min}$, facilitated the enhanced memory performance. However, these results should be interpreted with caution, since we did not include any memory tests in the present study. The effect of DBS (20 min) on acetylcholine release seemed to decline with continued stimulation. This transient effect can be explained by exhaustion of the acetylcholine pools that may take place after an enhanced release for more than $20 \mathrm{~min}$. Thus, chronic DBS might eventually be associated with acetylcholine depletion. In this case, either intermittent or closed-loop stimulation [23] when applying chronic DBS of the fornix might be necessary to maintain beneficial effects on memory functions.

\section{ACKNOWLEDGEMENTS}

This study was funded by the Internationale Stichting Alzheimer Onderzoek (ISAO). A travel grant for experiments conducted at Oxford University was awarded by Boehringer Ingelheim. 


\section{REFERENCES}

1. Batsch N, Mittelman M. World Alzheimer Report 2012: Overcoming the stigma of dementia. 2012.

2. Rosini M, Simoni E, Bartolini M, Cavalli A, Ceccarini L, Pascu N, et al. Inhibition of acetylcholinesterase, beta-amyloid aggregation, and NMDA receptors in Alzheimer's disease: a promising direction for the multi-target-directed ligands gold rush. Journal of medicinal chemistry. 2008;51(15):4381-4.

3. Thies W, Bleiler L. 2011 Alzheimer's disease facts and figures. Alzheimers Dement. 2011;7(2):208-44. Epub 2011/03/19. eng.

4. Hamani C, McAndrews MP, Cohn M, Oh M, Zumsteg D, Shapiro CM, et al. Memory enhancement induced by hypothalamic/fornix deep brain stimulation. Annals of Neurology. 2008;63(1):119-23.

5. Hescham S, Lim LW, Jahanshahi A, Steinbusch HW, Prickaerts J, Blokland A, et al. Deep brain stimulation of the forniceal area enhances memory functions in experimental dementia: the role of stimulation parameters. Brain Stimul. 2013;6(1):72-7. Epub 2012/03/13. eng.

6. Laxton AW, Tang-Wai DF, McAndrews MP, Zumsteg D, Wennberg R, Keren R, et al. A phase I trial of deep brain stimulation of memory circuits in Alzheimer's disease. Annals of Neurology. 2010;68(4):521-34.

7. Hescham S, Temel Y, Casaca-Carreira J, Arslantas K, Yakkioui Y, Blokland A, et al. A neuroanatomical analysis of the effects of a memory impairing dose of scopolamine in the rat brain using cytochrome c oxidase as principle marker. J Chem Neuroanat. 2014;59-60:1-7.

8. Budzikowski AS, Vahid-Ansari F, Leenen FH. Chronic activation of brain areas by high-sodium diet in Dahl salt-sensitive rats. The American journal of physiology. 1998;274(6 Pt 2):H2046-52.

9. Jahanshahi A, Schonfeld L, Janssen ML, Hescham S, Kocabicak E, Steinbusch HW, et al. Electrical stimulation of the motor cortex enhances progenitor cell migration in the adult rat brain. Experimental brain research. 2013;231(2):165-77.

10. Klinkenberg I, Sambeth A, Blokland A. Acetylcholine and attention. Behav Brain Res. 2011;221(2):43042.

11. Micheau J, Marighetto A. Acetylcholine and memory: a long, complex and chaotic but still living relationship. Behav Brain Res. 2011;221(2):424-9.

12. Paxinos G, Watson C. The rat brain in steroetaxic coordinates. 1998.

13. Somogyi P, Takagi H. A note on the use of picric acid-paraformaldehyde-glutaraldehyde fixative for correlated light and electron microscopic immunocytochemistry. Neuroscience. 1982;7(7):1779-83.

14. Fontaine D, Deudon A, Lemaire JJ, Razzouk M, Viau P, Darcourt J, et al. Symptomatic treatment of memory decline in Alzheimer's disease by deep brain stimulation: a feasibility study. J Alzheimers Dis. 2013;34(1):315-23. Epub 2012/11/22. eng.

15. Farovik A, Dupont LM, Eichenbaum H. Distinct roles for dorsal CA3 and CA1 in memory for sequential nonspatial events. Learning \& Memory. 2010;17(1):12-7.

16. Lee I, Jerman TS, Kesner RP. Disruption of delayed memory for a sequence of spatial locations following CA1- or CA3-lesions of the dorsal hippocampus. Neurobiology of Learning and Memory. 2005;84(2):138-47.

17. West MJ, Kawas CH, Stewart WF, Rudow GL, Troncoso JC. Hippocampal neurons in pre-clinical Alzheimer's disease. Neurobiol Aging. 2004;25(9):1205-12.

18. Francis PT. The interplay of neurotransmitters in Alzheimer's disease. CNS spectrums. 2005;10(11 Suppl 18):6-9.

19. Morris RGM, Anderson E, Lynch GS, Baudry M. Selective impairment of learning and blockade of long-term potentiation by an N-methyl-D-aspartate receptor antagonist, AP5. Nature. 1986;319(6056):774-6. 
20. Lau A, Tymianski M. Glutamate receptors, neurotoxicity and neurodegeneration. Pflugers Arch - Eur J Physiol. 2010;460(2):525-42. English.

21. Narimatsu N, Harada N, Kurihara H, Nakagata N, Sobue K, Okajima K. Donepezil Improves Cognitive Function in Mice by Increasing the Production of Insulin-Like Growth Factor-I in the Hippocampus. Journal of Pharmacology and Experimental Therapeutics. 2009;330(1):2-12.

22. Barker GRI, Warburton EC. When Is the Hippocampus Involved in Recognition Memory? The Journal of Neuroscience. 2011;31(29):10721-31.

23. Rosin B, Slovik M, Mitelman R, Rivlin-Etzion M, Haber Suzanne N, Israel Z, et al. Closed-loop deep brain stimulation is superior in ameliorating Parkinsonism. Neuron. 2011;72(2):370-84. 



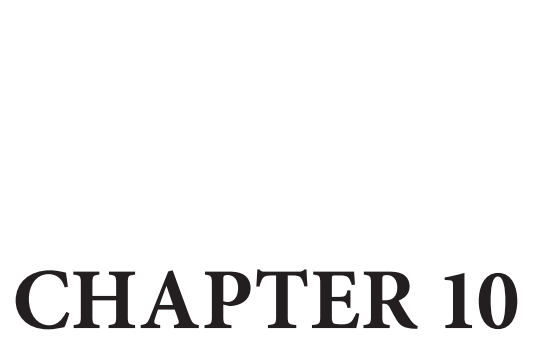
FORNIX DEEP BRAIN STIMULATION INDUCED LONG-TERM SPATIAL MEMORY INDEPENDENT OF HIPPOCAMPAL NEUROGENESIS

Sarah Hescham, Yasin Temel, Sandra Schipper, Arjan Blokland, Ali Jahanshahi 


\section{ABSTRACT}

Deep brain stimulation (DBS) is an established symptomatic treatment modality for movement disorders and constitutes an emerging therapeutic approach for the treatment of memory impairment. In line with this, fornix DBS has shown to ameliorate cognitive decline associated with dementia. Nonetheless, mechanisms mediating clinical effects in demented patients or patients with other neurological disorders are largely unknown. There is evidence that DBS is able to modulate neurophysiological activity in targeted brain regions. We therefore hypothesized that DBS might be able to influence cognitive function via activity-dependent regulation of hippocampal neurogenesis. Using stimulation parameters, which were validated to restore memory loss in a previous behavioural study, we here assessed long-term effects of fornix DBS. In order to do so, we injected the thymidine analogue, bromodeoxyuridine (BrdU) after DBS and perfused animals 6.5 weeks later. A week prior to perfusion, memory performance was assessed in the water maze. We found that acute stimulation of the fornix improved spatial memory performance in the water maze when the probe trial was performed 1 hour after the last training session. However, no evidence for stimulation-induced neurogenesis was found in fornix DBS rats when compared to sham. Our results suggest, that fornix DBS improves memory function, independent of hippocampal neurogenesis, possibly through other mechanisms such as synaptic plasticity and acute neurotransmitter release. 


\section{INTRODUCTION}

Deep brain stimulation (DBS) by permanently implanted electrodes in the brain is a popular treatment. Up to now, DBS has provided treatment for more than 130,000 patients, suffering from Parkinson's disease, essential tremor, and dystonia worldwide [1-3]. Despite its widespread use, the precise mechanism of action of DBS therapy remains unknown. Clinical applications of DBS in neuropsychiatric diseases including memory impairment are in the early stages or under investigation $[4,5]$. The fornix has gained growing attention as potential DBS target to alleviate memory impairments [68]. In a phase 1 clinical trial, the effects of forniceal stimulation in Alzheimer's disease (AD) patients were assessed [8]. Although some patients showed improvement and slowing in the rate of cognitive decline at 6 and 12 months, others did not respond to the therapy. It was reported recently, that in $2 \mathrm{AD}$ patients with best clinical response of this study, bilateral fornix DBS of $130 \mathrm{~Hz}, 3-3.5 \mathrm{~V}$ and $90 \mu$ s pulse width increased hippocampal volume [9]. This hippocampal volume change strongly correlated with hippocampal metabolism and a volume change in the fornix and mammillary bodies, suggesting a circuit-wide effect of stimulation.

Besides inducing morphological changes, fornix DBS might also have an effect on adult neurogenesis. Throughout life, new neurons are continuously generated in the granule cell layer of the dentate gyrus and in the subventricular zone. Most of these adult-generated dentate granule cell neurons are thought to contribute to the formation of hippocampus-dependent memory [10,11]. A few studies in rodents have already provided evidence that DBS of the anterior thalamic nucleus and entorhinal cortex, promotes hippocampal neurogenesis by increasing the cell proliferation and survival of newly generated neurons in the dentate gyrus [12-14]. In these studies, rats or mice were first treated with electrical stimulation in the target region, then injected with the cell-proliferation marker bromodeoxyuridine (BrdU) and sacrificed after a few weeks. Increased neurogenesis could furthermore be linked to improved spatial memory in the water maze [13].

In the present study, we also investigated whether fornix DBS induces long-term neurogenic changes in the dentate gyrus, by injecting BrdU after DBS. Subsequent to the completion of a hippocampus-dependent memory task, we analyzed the hippocampi 6.5 weeks after fornix DBS and labeled them with antibodies to BrdU and the neuronal marker, NeuN. 


\section{MATERIALS AND METHODS}

\section{Subjects}

Sprague Dawley (SD) rats from Charles River (Sulzfeld, Germany) were used, their weight ranging between 280-300 $\mathrm{g}$ at time of surgery. The temperature of the colony room was maintained at a temperature of $21 \pm 1{ }^{\circ} \mathrm{C}$. Rats were housed 2 or 3 per cage with rat chow and water available ad libitum in a reversed 12:12 h light-dark cycle. For the time of experimental procedures (stimulation and BrdU injections) rats were housed individually, but were re-grouped thereafter. All experimental animal procedures were executed during the dark phase.

\section{Experimental groups}

Rats were randomly assigned to one of the following experimental groups: $\operatorname{sham}(n=7)$ or fornix DBS $(\mathrm{n}=10)$. We included more rats in the fornix DBS group in order to account for factors such as loss of electrodes or incorrect electrode placements.

\section{Surgical procedure}

The exact surgical procedure has been described elsewhere [15]. In short, DBS electrodes were implanted in the fornix using a rodent stereotactic apparatus (Stoelting, Wood Dale, IL, USA, model 51653). The rat skull was exposed under Isoflurane anaesthesia (IsoFlo ${ }^{\oplus}$, Abbott Laboratories Ltd, Berkshire, Great Britain) and two burr holes for bilateral DBS electrodes were made at the level of the fornix (coordinates from bregma according to the rat brain atlas of Paxinos and Watson [16]: AP: $-1.8 \mathrm{~mm}$; ML: $1.3 \mathrm{~mm}$; DV: $-8.0 \mathrm{~mm}$ ). The construct was anchored with dental cement (Paladur, Heraeus Kulzer GmbH, Hanau, Germany). Sham rats underwent the same electrode implantations, but were not stimulated.

\section{Deep brain stimulation}

DBS was applied for $4 \mathrm{~h}$ at $100 \mathrm{~Hz}, 100 \mu \mathrm{A}$ and $100 \mu$ s pulse width using a World Precision Instrument digital stimulator (DS8000, WPI, Berlin, Germany) and two stimulus isolators (DLS100, WPI, Berlin, Germany). Sham animals were only attached to cables and not stimulated.

\section{BrdU labeling}

BrdU was used to identify cells that were born after DBS. BrdU (Sigma-Aldrich) was dissolved in $0.9 \% \mathrm{NaCl}$ ( $\mathrm{pH} 7.6$ ) to $8 \mathrm{mg} / \mathrm{ml}$. Three days after DBS, all rats were injected intraperitoneally twice daily ( $8 \mathrm{~h}$ apart) with $50 \mathrm{mg} / \mathrm{kg}$ BrdU for 3 consecutive days. The interval between DBS and onset of BrdU injection was chosen based on a previous 
study, in which proliferative activity evaluated by BrdU in the dentate gyrus reached a plateau 3-5 days after DBS [13].

\section{Water maze}

The water maze consists of a circular black polyethylene tank (diameter $153 \mathrm{~cm}$ ) with $63 \mathrm{~cm}$ high walls. The pool was filled with $40 \mathrm{~cm}$ of water, which was maintained at 22 $\pm 1{ }^{\circ} \mathrm{C}$ and made opaque by adding black, nontoxic paint. The black escape platform (diameter $11 \mathrm{~cm}$ ), was submerged $1.5 \mathrm{~cm}$ below the surface of the water. A video camera was mounted in the center above the pool and registered movements of the rat (Ethovision Pro, Noldus, The Netherlands).

In general, the animals received 4 trials during 5 acquisition sessions, which were given on 5 consecutive days (total of 20 swim trials). Different start locations were used and tracing began when the animal was released into the pool facing the wall of the tank. Rats were given $60 \mathrm{~s}$ to reach the platform and if the rat failed to find the platform within $60 \mathrm{~s}$, the experimenter guided the rat to the target. The time between subsequent trials was about $10 \mathrm{~min}$. A probe trial was given $1 \mathrm{~h}$ and $48 \mathrm{~h}$ after the last trial on the 5 th acquisition session.

In the reversal learning paradigm the platform was moved to a novel location and the rats were again trained for 2 consecutive days with 4 trials/day. Rats were subjected to the reversal probe trial, $1 \mathrm{~h}$ after the last training session.

\section{Tissue collection}

At the end of the experiments, the rats were overdosed with pentobarbital (Apotheek Faculteit Diergeneeskunde, Utrecht, The Netherlands). Transcardial perfusions with Tyrode and then Somogyi fixative solution (4\% paraformaldehyde, picric acid, PBS, glutaraldehyde) were carried out. Brains were removed and placed in fresh fixative (identical composition as Somogyi solution, but lacking glutaraldehyde) at $4^{\circ} \mathrm{C}$ for 2 hours. Subsequently brains were transferred to $1 \% \mathrm{NaN}_{3}$ at $4{ }^{\circ} \mathrm{C}$ for long-term storage.

For vibratome sectioning (Leica ${ }^{\star}$, Wetzlar, Germany), brains were embedded in $10 \%$ gelatin from porcine skin (Sigma-Aldrich, Zwijndrecht, The Netherlands), and then cut into $30 \mu \mathrm{m}$ slices in the frontal plane. Slices were immediately transferred into $1 \%$ $\mathrm{NaN}_{3}$ and kept at $4^{\circ} \mathrm{C}$.

\section{Immunohistochemistry}

A double-immunofluorescent BrdU/NeuN staining was performed for sections containing the hippocampus in 7 fornix DBS and 7 sham animals. For BrdU detection, DNA denaturation was conducted by incubating for $2 \mathrm{~h}$ in $50 \%$ formamide at $65^{\circ} \mathrm{C}$, followed by washing and $30 \mathrm{~min}$ in $2 \mathrm{~N} \mathrm{HCL}$ at $37^{\circ} \mathrm{C}$. After blocking, sections were incubated with mouse monoclonal anti-BrdU (1:100; Sigma-Aldrich, Zwijndrecht, 
Netherlands) overnight at $4{ }^{\circ} \mathrm{C}$. Subsequently, donkey-anti-mouse secondary antibody (1:100; Alexa 488, Invitrogen, Carlsbad, CA) was applied. Incubation with biotinylated NeuN (1:100; Chemicon, Temecula, CA) was carried out for 3 days at $4{ }^{\circ} \mathrm{C}$, followed by streptavidine 594 (1:1000 Invitrogen, Carlsbad, CA). Lastly, brain sections were mounted and coverslipped with $80 \%$ glycerol.

The number of BrdU/NeuN double-labeled cells was counted using the stereological procedure, optical fractionator. Counts were done using a confocal microscope (DSU, Olympus $^{\oplus}$ BX51W1), a motorized stage, and the StereoInvestigator software (MicroBrightField, Williston, VT). The granule cell layer of the dentate gyrus was defined as the region of interest. All double-labelled BrdU/NeuN cells in an average of 6 sections, $300 \mu \mathrm{m}$ apart were counted with a $60 \mathrm{X}$ objective. The chosen brain sections extended from bregma $-3.1 \mathrm{~mm}$ to bregma $-4.9 \mathrm{~mm}$. The total number of positive cells was estimated as a function of the number of cells counted and the sampling probability [17].

\section{Verification of electrode placements}

Sections containing electrode trajectories from all animals were mounted on gelatin coated glass slides. A standard haematoxylin-eosin staining was employed in order to inspect the sections under bright field microscopy.

\section{Statistical analyses}

Statistical analysis was performed using SPSS (SPSS Inc., Chicago, IL, USA). Acquisition data of water maze trials were analyzed by repeated measures ANOVA. Probe trials and immunohistochemical findings were analyzed using an independent samples t-test. All p-values less than 0.05 were considered statistically significant.

\section{RESULTS}

\section{Verification of electrode placements}

DBS electrodes were all correctly located in the vicinity of the fornix. The electrode trajectory in one animal of the stimulated group was directed ventral of the fornix, but since the rat showed similar behaviour to other stimulated animals, we expected that the fornix was within the electric field and included the rat in the analysis.

\section{Water maze}

DBS rats received fornix stimulation and were trained in the water maze 8 days before perfusion took place. Sham rats were treated identically, except that no current was delivered. During the acquisition phase, latency to find the platform declined similarly in both groups $(\mathrm{F}(1,66)=0.658$; n.s., Fig. 1). One hour after completion of the train- 


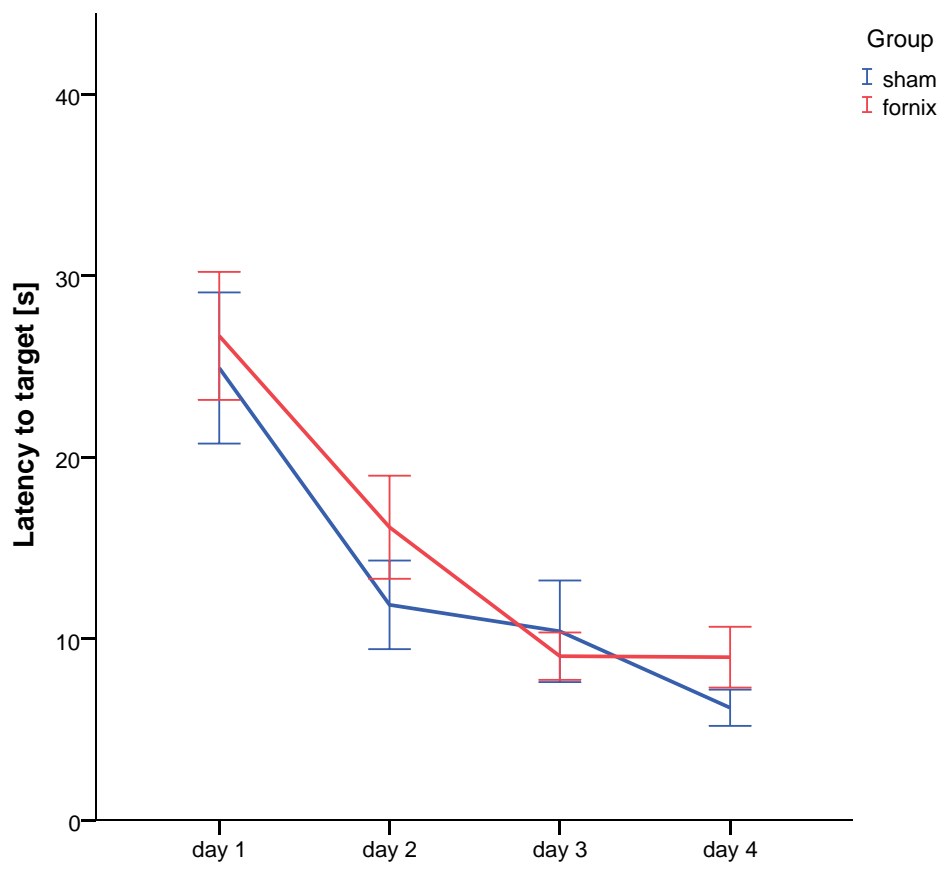

Figure 1: Acquisition trials of fornix DBS and sham animals. No difference in latency to find the platform was found between the groups. Data represents mean \pm S.E.M.

ing, spatial memory was assessed in a probe trial. In this test, both DBS and sham rats spent similar time in the target zone $(\mathrm{t}(15)=1.241$; n.s, Fig. $2 \mathrm{~A})$. However, only fornix DBS rats crossed the former platform location more frequently when compared to sham $(t(15)=2.209 ; \mathrm{p}<0.05$, Fig. $2 \mathrm{~B})$, indicating that fornix stimulation facilitated short-term spatial memory. At the $48 \mathrm{~h}$ probe test, no difference between time spent and number of crossings to the target location was found. In the reversal training both groups performed equally well and there was also no statistical difference between the groups in the reversal probe trial which took place $1 \mathrm{~h}$ after the last reversal training session (all t's $\leq 0.462$; n.s.).

\section{BrdU/NeuN cell counts}

There were similar numbers of BrdU/NeuN double-labeled cells in both fornix DBS and sham groups $(\mathrm{t}(12)=0.522$, n.s.), and no additional differences between the anterior, medial and posterior portions of the dentate gyrus (all t's $\leq 0.985$; n.s., Fig. 3 ). 

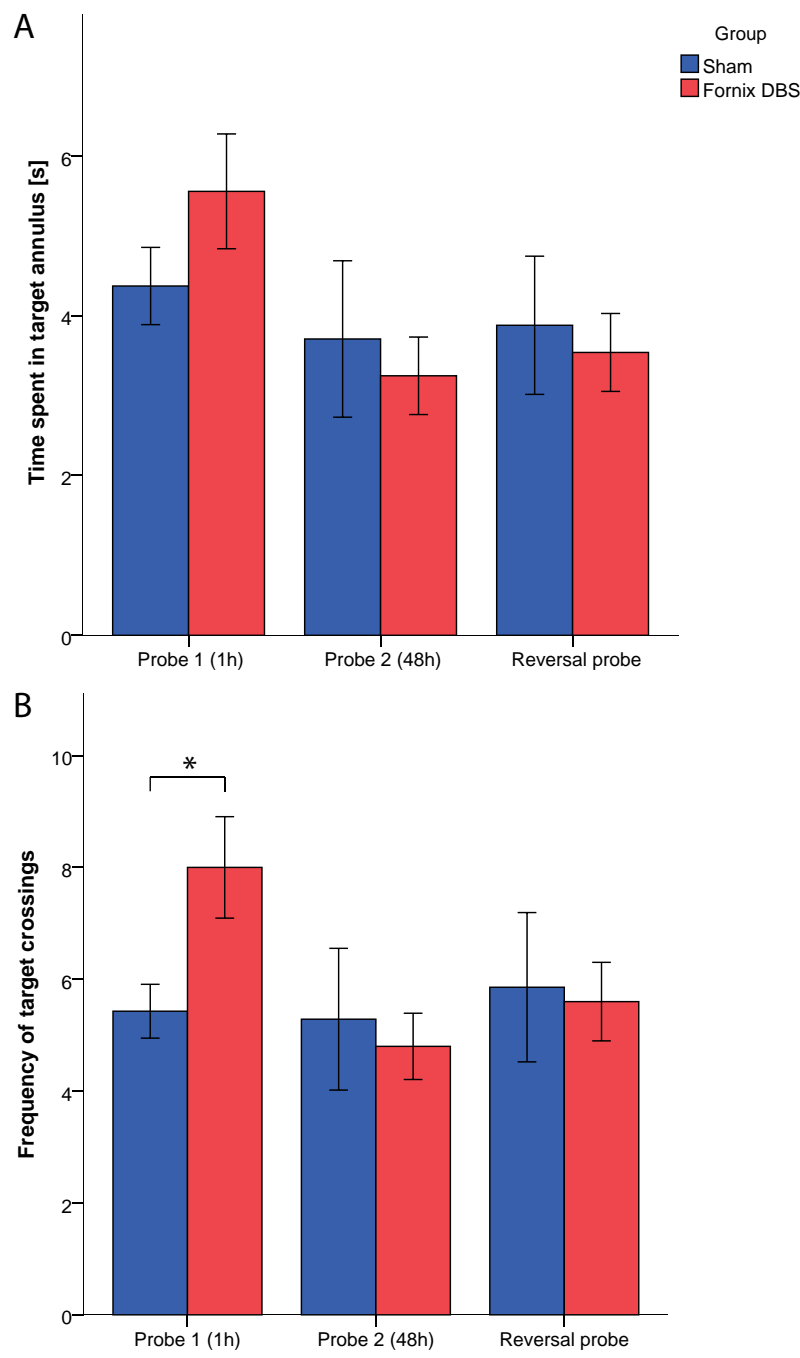

Figure 2: Effects of fornix DBS during the different probe trials (after $1 \mathrm{~h}$ or $48 \mathrm{~h}$ delay and in the probe trial $1 \mathrm{~h}$ after the reversal training) when compared to sham. There was no difference between the groups in time spent in the target annulus (A). Fornix DBS rats, however, crossed the target annulus more often than sham animals $(\mathrm{B}) .{ }^{*}$ indicates $\mathrm{p}<0.05$. Data represents mean \pm S.E.M.

\section{DISCUSSION}

Adult hippocampal neurogenesis has long been implicated as important for learning and memory [11]. Consequently, the role of neurogenesis in the adult brain might open up important opportunities for the development of therapeutic interventions, which could moderate or delay disorders with memory decline. Studies in rodents have 


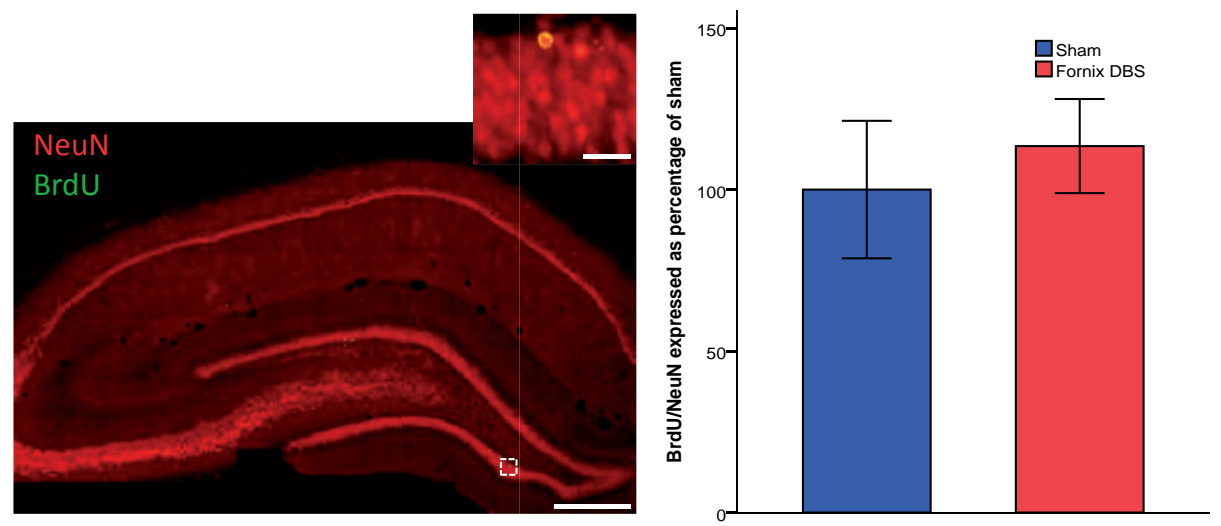

Figure 3: Representative picture (scale bar $=500 \mu \mathrm{m}$ ) of a hippocampal section stained for NeuN (red) and BrdU (green) with a high-power inset on the top right showing a double-labeled cell (scale bar = $50 \mu \mathrm{m})$. Graph represents number of double-labeled BrdU/NeuN cells in the dentate gyrus expressed as percentage of sham \pm S.E.M. There was no significant difference between the groups.

demonstrated that DBS of the anterior thalamic nucleus or entorhinal cortex increases cell proliferation in the dentate gyrus $[13,14]$. These targets, however, have not yet been explored as potential treatment for dementia. DBS of the fornix, on the other hand, has already been reported in a case study [7] and phase I clinical trial [8], to slow down progression of memory loss in individuals with early Alzheimer's disease. The exact mechanisms of action are yet to be defined. Previously, we have also found superior memory performance of fornix DBS rats in an experimental model of dementia [18]. We were able to link restoration of memory loss to an increased neuronal activation in the CA1 and CA3 subfield of the dorsal hippocampus, as well as increased hippocampal acetylcholine levels (chapter 9). The hippocampus receives abundant cholinergic innervation from the basal forebrain and it has been shown that selective neurotoxic lesion of the basal forebrain reduces neurogenesis in the dentate gyrus and thus impairs spatial memory [19]. In the same study systemic administration of the cholinergic agonist physostigmine was able to increase neurogenesis in the dentate gyrus. In contrast to these studies, we did not find any evidence for enhanced neurogenesis following fornix DBS. Although no increased neurogenesis was observed, the stimulated rats visited the site of platform location more frequently than sham animals in the $1 \mathrm{~h}$ probe trial delay.

It has been shown that hippocampal acetylcholine primarily influences proliferation and/or short-term survival rather than long-term survival or differentiation of new neurons [19]. It might therefore be possible, that our experimental paradigm, which allowed new-born cells to mature for 6 weeks, was too long to depict differences between fornix DBS and sham animals. Indeed, in an unpublished data set, we stained brains of fornix DBS and sham animals from a previous behavioral study [18] for doublecortin 
and found clear evidence for enhanced proliferative activity in the dentate gyrus of stimulated rats. Nonetheless, short-term neurogenic differences might not explain the enhanced memory performance after 6 weeks of stimulation. We therefore speculate that fornix DBS might have had an effect on other neuroplastic mechanisms, for instance synaptic potentiation. It has been suggested before, that changes in proliferative activity is not necessarily a key factor determining the efficacy of synaptic potentiation [20]. In line with this, it was shown that synaptic potentiation was restored within 6 weeks in a transgenic mouse model of suppressed neurogenesis [21].

In conclusion, our results suggest that acute fornix DBS improves long-term spatial memory independently from DBS-induced neurogenesis, but possibly by increasing the connectivity among existing neurons. Further studies are indicated, which investigate the effects of fornix DBS on long-term potentiation and long-term depression. 


\section{REFERENCES}

1. Benabid AL, Pollak P, Hoffmann D, Gervason C, Hommel M, Perret JE, et al. Long-term suppression of tremor by chronic stimulation of the ventral intermediate thalamic nucleus. The Lancet. 1991;337(8738):403-6.

2. Blond S, Siegfried J. Thalamic stimulation for the treatment of tremor and other movement disorders. Acta neurochirurgica Supplementum. 1991;52:109-11.

3. Holloway KL, Baron MS, Brown R, Cifu DX, Carne W, Ramakrishnan V. Deep Brain Stimulation for Dystonia: A Meta-Analysis. Neuromodulation: Technology at the Neural Interface. 2006;9(4):253-61.

4. Temel Y, Hescham SA, Jahanshahi A, Janssen ML, Tan SK, van Overbeeke JJ, et al. Neuromodulation in psychiatric disorders. International review of neurobiology. 2012;107:283-314.

5. Holtzheimer PE, Mayberg HS. Deep Brain Stimulation for Psychiatric Disorders. Annual Review of Neuroscience. 2011;34(1):289-307.

6. Hamani C, McAndrews MP, Cohn M, Oh M, Zumsteg D, Shapiro CM, et al. Memory enhancement induced by hypothalamic/fornix deep brain stimulation. Annals of Neurology. 2008;63(1):119-23.

7. Fontaine D, Deudon A, Lemaire JJ, Razzouk M, Viau P, Darcourt J, et al. Symptomatic treatment of memory decline in Alzheimer's disease by deep brain stimulation: a feasibility study. J Alzheimers Dis. 2013;34(1):315-23. Epub 2012/11/22. eng.

8. Laxton AW, Tang-Wai DF, McAndrews MP, Zumsteg D, Wennberg R, Keren R, et al. A phase I trial of deep brain stimulation of memory circuits in Alzheimer's disease. Annals of Neurology. 2010;68(4):521-34.

9. Sankar T, Chakravarty MM, Bescos A, Lara M, Obuchi T, Laxton AW, et al. Deep Brain Stimulation Influences Brain Structure in Alzheimer's Disease. Brain Stimul. 2014;in press.

10. Shors TJ. From Stem Cells to Grandmother Cells: How Neurogenesis Relates to Learning and Memory. Cell Stem Cell. 2008;3(3):253-8.

11. Deng W, Aimone JB, Gage FH. New neurons and new memories: how does adult hippocampal neurogenesis affect learning and memory? Nature reviews Neuroscience. 2010;11(5):339-50.

12. Encinas JM, Hamani C, Lozano AM, Enikolopov G. Neurogenic hippocampal targets of deep brain stimulation. The Journal of Comparative Neurology. 2011;519(1):6-20.

13. Stone SSD, Teixeira CM, DeVito LM, Zaslavsky K, Josselyn SA, Lozano AM, et al. Stimulation of Entorhinal Cortex Promotes Adult Neurogenesis and Facilitates Spatial Memory. The Journal of Neuroscience. 2011;31(38):13469-84.

14. Toda H, Hamani C, Fawcett AP, Hutchison WD, Lozano AM. The regulation of adult rodent hippocampal neurogenesis by deep brain stimulation. J Neurosurg. 2008;108(1):132-8.

15. Tan S, Vlamings R, Lim L, Sesia T, Janssen ML, Steinbusch HW, et al. Experimental deep brain stimulation in animal models. Neurosurgery. 2010;67(4):1073-9. Epub 2010/10/01. eng.

16. Paxinos $\mathrm{G}$, Watson $\mathrm{C}$. The rat brain in stereotaxic coordinates: hard cover edition: Academic press; 2006.

17. Schmitz C, Hof PR. Recommendations for straightforward and rigorous methods of counting neurons based on a computer simulation approach. Journal of Chemical Neuroanatomy. 2000;20(1):93-114.

18. Hescham S, Lim LW, Jahanshahi A, Steinbusch HW, Prickaerts J, Blokland A, et al. Deep brain stimulation of the forniceal area enhances memory functions in experimental dementia: the role of stimulation parameters. Brain Stimul. 2013;6(1):72-7. Epub 2012/03/13. eng.

19. Mohapel P, Leanza G, Kokaia M, Lindvall O. Forebrain acetylcholine regulates adult hippocampal neurogenesis and learning. Neurobiology of Aging. 2005;26(6):939-46. 
20. Krugers HJ, van der Linden S, van Olst E, Alfarez DN, Maslam S, Lucassen PJ, et al. Dissociation between apoptosis, neurogenesis, and synaptic potentiation in the dentate gyrus of adrenalectomized rats. Synapse. 2007;61(4):221-30.

21. Singer BH, Gamelli AE, Fuller CL, Temme SJ, Parent JM, Murphy GG. Compensatory network changes in the dentate gyrus restore long-term potentiation following ablation of neurogenesis in young-adult mice. Proceedings of the National Academy of Sciences. 2011;108(13):5437-42. 




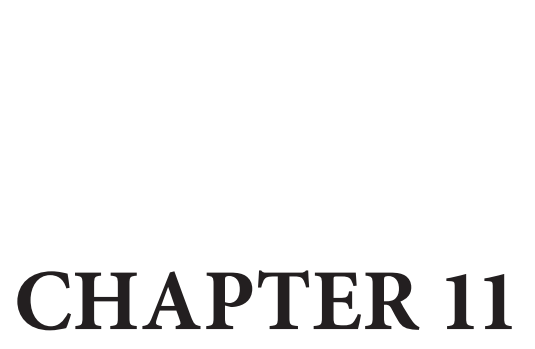
GENERAL DISCUSSION AND CONCLUSION 


\section{GENERAL DISCUSSION}

Cognitive disorders are a major societal burden. In an ever-growing and aging population, these conditions demand urgent attention, in order to alleviate healthcare resources as well as provide a good quality of life for patients and caregivers. Although memory impairments are relatively common conditions in the elderly, few treatments are available to arrest or slow down the progression of memory loss. By targeting specific structures within known circuits, deep brain stimulation (DBS) can have beneficial effects across memory and cognitive networks, and is therefore a potentially promising avenue for non-drug based dementia treatments.

The application of DBS in psychiatry has emerged in the past 50 years [1-3]. To understand state-of-the-art applications of DBS in psychiatric conditions which potentially include memory impairments, I have reviewed relevant clinical studies on neuromodulation (Chapter 2). Non-invasive neuromodulation therapies, such as transcranial magnetic stimulation (TMS) and transcranial direct current stimulation (tDCS), can produce short-term beneficial effects on refractory psychiatric symptoms $[4,5]$. However, the effects on long-term treatment modalities are unknown. A main disadvantage seems to be that transcranial stimulation mainly targets cortical structures and does not reach deeply situated brain regions. Deep brain stimulation, on the contrary, enables focused stimulation of specific brain targets. At present, an increasing number of studies investigate the safety and efficacy of DBS, showing significant therapeutic effects in patients with different refractory psychiatric disorders [6].

With regard to memory-related disorders, DBS of the fornix and nucleus basalis of Meynert have been investigated as potential treatments for dementia in clinical trials (Chapter 3). The studies that were carried out, however, were mostly safety studies, with small sample sizes and no control group [7, 8]. As a result, this encouraging therapy has not been approved in the clinics, yet. In order to conduct standardized clinical trials, more preclinical research is warranted. Preclinical animal models are crucial to examine relevant brain targets and can accelerate the development of clinical applications of DBS. In addition, animal models are essential to increase our understanding of the underlying neural substrates of memory enhancement after DBS [3].

To further investigate the behavioral effects and mechanisms of DBS, we used a scopolamine-induced rat model of dementia. Scopolamine, a non-selective acetylcholine antagonist, has been used as a standard drug to induce memory impairments associated with aging and dementia in animals and humans [9]. Chronic scopolamine administration indeed has a widespread effect on brain depressant activity, which corroborates the variety of behavioral effects of this drug (Chapter 4). The dose I chose for the subsequent behavioral studies has been validated to impair recognition and spatial memory [9]. 
Thus, I have used the scopolamine-induced rat model of memory impairment and applied DBS to one of the potential targets in dementia-treatment, namely the fornix (Chapter 5). The fornices are white matter bundles, which reciprocally connect the bilateral hippocampi to the septal nuclei, nucleus accumbens (precommissural fornix) and hypothalamus (postcommissural fornix) [10]. In this study, I found that DBS of the fornix was able to restore memory loss in a spatial memory task when specific set of stimulation parameters was used. In particular, DBS of the fornix was not frequency dependent, but rather dependent on high current densities. With the investigated stimulation parameters, I found no evidence on anxiety-related side effects [11].

I have also investigated the efficacy of DBS in an aged animal model (Chapter 6). In particular, I aimed to investigate DBS of the input and output of the hippocampus and have stimulated the fornix and entorhinal cortex, respectively. In 24-months-old rats, I found that DBS of the fornix improved short-term memory in a spatial memory task. Entorhinal cortex DBS had beneficial effects on long-term recognition memory. Again no evidence for anxiety- related side effects were found. Taken together, these results support the notion that the fornix and entorhinal cortex are involved in different memory domains. This is in line with lesion studies, showing the involvement of the fornix in spatial [12-14] and the entorhinal cortex in recognition memory $[15,16]$, respectively.

Next, I assessed whether DBS can have beneficial memory effects when applied to other structures within the circuit of Papez (Chapter 7). Therefore, I implanted electrodes in the CA1 subregion of the hippocampus, the mammillothalamic tract, anterior thalamic nucleus and enthorhinal cortex and again made use of the scopolamine-induced rat model of dementia. DBS of the CA1 and entorhinal cortex was able to restore memory loss when specific set of stimulation parameters were used. The conclusions, however, were twofold. Firstly, I have shown that within the circuit of Papez, only DBS of the CA1 subregion, entorhinal cortex and fornix is able to restore spatial memory loss. Nonetheless, secondly, from a stereotactic point of view, I concluded that a strategic target should be chosen for therapy. The hippocampus and entorhinal cortex are relatively large brain regions in rodents and primates [17]. Therefore, neuromodulation through a single electrode might be challenging, given the risk of not targeting the relevant region in a patient. Contrary to this, the fornix entails localized fiber bundles and its size is accessible in both, rodents and primates. Thus, within the circuit of Papez, the fornix might consequently represent a preferred target for the treatment of dementia-related disorders with DBS.

In the last 3 chapters of this thesis, I focused on mechanisms of action of DBS. In Chapter 8, I investigated brains of rats, which underwent DBS in the entorhinal cortex, mammillothalamic tract and anterior thalamic nucleus. When looking at dopamine, a neurotransmitter widely acknowledged as important for memory functions, I have not 
found any difference between sham and entorhinal cortex DBS animals in the ventral tegmental area. Only DBS of the anterior thalamic nucleus, induced substantial higher numbers of TH-immunoreactive cells in the ventral tegmental area, while there was no difference in the number activated cells (measured by c-Fos). My findings suggest that DBS can induce a phenotypic switch, or GABA - dopamine neurotransmitter respecification, in the ventral tegmental area [18].

With regard to fornix DBS, mechanisms of action were evaluated by immunohistochemistry and microdialysis experiments (Chapter 9). First, I performed c-Fos immunohistochemistry in the dorsal hippocampus and found that beneficial memory performance was accompanied with a selective activation of cells in the CA1 and CA3 subfields. In order to elucidate what types of neurons were activated in the hippocampus, additional rats were implanted with fornix DBS electrodes and hippocampal microdialysis probes. During stimulation, I observed a substantial increase in the levels of extracellular hippocampal acetylcholine in fornix DBS rats when compared to sham. Specifically, acetylcholine levels increased in the first $20 \mathrm{~min}$ of stimulation. No effects on hippocampal glutamate levels were found during or after DBS. Therefore, my findings provide first experimental evidence that beneficial memory effects following fornix DBS can be attributed to cholinergic modulation of the hippocampus.

Lastly, I assessed whether acute DBS of the fornix can produce long-term changes in memory and whether they can be linked to adult hippocampal neurogenesis (Chapter 10). Although, fornix stimulated rats showed superior memory performance 6 weeks after DBS when compared to sham, I did not find evidence for increased hippocampal neurogenesis. My findings suggest, that besides yielding immediate effects on memory (e.g., vivid autobiographical recall seen during initiation of stimulation in some clinical studies $[8,19]$ and improved memory performance in rodents [11]), fornix DBS might also induce long-term memory effects. These long-term effects, however, cannot be explained by neurogenesis and further studies are indicated, which investigate changes in other neuroplastic domains.

\section{CONCLUSION}

Clinical studies have shown that targeting key structures in memory and cognitive circuits for DBS therapy is safe and can activate these circuits. In particular, case studies or phase I trials of patients with Alzheimer's disease or other dementias suggest that DBS may be associated with stabilization or improvement in memory. In the present thesis, I have found the fornix to be the most optimal target structure within the circuit of Papez to improve short-term spatial memory. DBS of the fornix induces neuronal activation in the CA1 and CA3 subfields of the hippocampus and also enhances hippocampal 
acetylcholine levels instantaneously. Moreover, acute fornix DBS produced long-term memory effects. Nonetheless, no evidence for stimulation-induced survival of newborn hippocampal neurons was found. Despite these findings, the exact mechanisms of action are yet to be explored. Moreover, the efficacy of nucleus basalis of Meynert DBS, which highlights another promising target in the treatment of Alzheimer's disease [20] needs further investigation. Also, the outcome of long-term DBS in animals could reveal interesting findings with respect to the effects on brain functions and the efficacy on memory enhancement. Ongoing preclinical work may help to find the most beneficial target structure and define the therapeutic value of DBS in dementia-related disorders. Ultimately, the outcomes of sham-controlled, randomized clinical trials will determine whether DBS will play a role in the management of memory and cognitive disorders. 


\section{REFERENCES}

1. Holtzheimer PE, Mayberg HS. Deep Brain Stimulation for Psychiatric Disorders. Annual Review of Neuroscience. 2011;34(1):289-307.

2. Goodman WK, Alterman RL. Deep Brain Stimulation for Intractable Psychiatric Disorders. Annual Review of Medicine. 2012;63(1):511-24.

3. Hamani C, Temel Y. Deep Brain Stimulation for Psychiatric Disease: Contributions and Validity of Animal Models. Sci Transl Med. 2012;4(142):142rv8.

4. Tortella G, Casati R, Aparicio LV, Mantovani A, Senco N, D’Urso G, et al. Transcranial direct current stimulation in psychiatric disorders. World journal of psychiatry. 2015;5(1):88-102.

5. Kim DR, Pesiridou A, O'Reardon JP. Transcranial magnetic stimulation in the treatment of psychiatric disorders. Current psychiatry reports. 2009;11(6):447-52.

6. Temel Y, Hescham SA, Jahanshahi A, Janssen ML, Tan SK, van Overbeeke JJ, et al. Neuromodulation in psychiatric disorders. International review of neurobiology. 2012;107:283-314.

7. Freund HJ, Kuhn J, Lenartz D, Mai JK, Schnell T, Klosterkoetter J, et al. Cognitive functions in a patient with Parkinson-dementia syndrome undergoing deep brain stimulation. Arch Neurol. 2009;66(6):781-5. Epub 2009/06/10. eng.

8. Laxton AW, Tang-Wai DF, McAndrews MP, Zumsteg D, Wennberg R, Keren R, et al. A phase I trial of deep brain stimulation of memory circuits in Alzheimer's disease. Annals of Neurology. 2010;68(4):521-34.

9. Klinkenberg I, Blokland A. The validity of scopolamine as a pharmacological model for cognitive impairment: A review of animal behavioral studies. Neuroscience \& Biobehavioral Reviews. 2010;34(8):1307-50.

10. Aggleton JP, O’Mara SM, Vann SD, Wright NF, Tsanov M, Erichsen JT. Hippocampal-anterior thalamic pathways for memory: uncovering a network of direct and indirect actions. Eur J Neurosci. 2010;31(12):2292-307. Epub 2010/06/17. eng.

11. Hescham S, Lim LW, Jahanshahi A, Steinbusch HW, Prickaerts J, Blokland A, et al. Deep brain stimulation of the forniceal area enhances memory functions in experimental dementia: the role of stimulation parameters. Brain Stimul. 2013;6(1):72-7. Epub 2012/03/13. eng.

12. Aggleton J, Keith A, Sahgal A. Both fornix and anterior thalamic, but not mammillary, lesions disrupt delayed non-matching-to-position memory in rats. Behav Brain Res. 1991;44(2):151-61.

13. Aggleton JP, Neave N, Nagle S, Hunt PR. A comparison of the effects of anterior thalamic, mamillary body and fornix lesions on reinforced spatial alternation. Behav Brain Res. 1995;68(1):91-101.

14. Dumont JR, Amin E, Wright NF, Dillingham CM, Aggleton JP. The impact of fornix lesions in rats on spatial learning tasks sensitive to anterior thalamic and hippocampal damage. Behav Brain Res. 2015;278(0):360-74.

15. Hunsaker MR, Chen V, Tran GT, Kesner RP. The medial and lateral entorhinal cortex both contribute to contextual and item recognition memory: A test of the binding ofitems and context model. Hippocampus. 2013;23(5):380-91.

16. Sauvage MM, Beer Z, Ekovich M, Ho L, Eichenbaum H. The Caudal Medial Entorhinal Cortex: a Selective Role in Recollection-Based Recognition Memory. The Journal of Neuroscience. 2010;30(46):15695-9.

17. Insausti R. Comparative anatomy of the entorhinal cortex and hippocampus in mammals. Hippocampus. 1993;3 Spec No:19-26. 
18. Dela Cruz JA, Hescham S, Adriaanse B, Campos FL, Steinbusch HW, Rutten BP, et al. Increased number of TH-immunoreactive cells in the ventral tegmental area after deep brain stimulation of the anterior nucleus of the thalamus. Brain structure \& function. 2014.

19. Hamani C, McAndrews MP, Cohn M, Oh M, Zumsteg D, Shapiro CM, et al. Memory enhancement induced by hypothalamic/fornix deep brain stimulation. Annals of Neurology. 2008;63(1):119-23.

20. Kuhn J, Hardenacke K, Lenartz D, Gruendler T, Ullsperger M, Bartsch C, et al. Deep brain stimulation of the nucleus basalis of Meynert in Alzheimer's dementia. Molecular psychiatry. 2014. 

VALORIZATION ADDENDUM 



\section{RELEVANCE FOR SOCIETY}

Today, more than 44.4 million people suffer from dementia [1]. This number will increase to about 135.5 million in 2050. Every year 7.7 million new cases of dementia are diagnosed, implying that there is a new case of dementia somewhere in the world every four seconds. Dementia has emerged as one of the leading health problems of our time and has been recently recognized as one of the major threats to world population [2]. Symptoms include progressive loss of memory, impaired reasoning and judgement, difficulties paying attention, communication problems and various non-cognitive symptoms, ultimately leading to disability and need for care.

Most individuals diagnosed with dementia are 65 years or older, although there is a growing awareness of cases that start before the age of 65 [1]. Demographic ageing is a worldwide process resulting from constantly improving health care systems. The fastest growth in the elderly population is taking place in China, India, and their South Asian and Pacific neighbors. In 2010, the total estimated worldwide cost of dementia was US $\$ 604$ billion, which equals to around $1 \%$ of the world's gross domestic product [3]. About $70 \%$ of the costs occur in Western Europe and North America. Statisticians even claim, that if dementia care would represent a country, it would be the world's 18th largest economy, ranking between Turkey and Indonesia [1].

Unfortunately, despite decades of research, we are still in need of an effective therapy for dementia, symptomatic or curative. There are 5 approved drugs on the market, which either modulate the cholinergic system by inhibiting acetylcholinesterase or reduce glutamate by antagonising specific glutamate receptors [4]. These pharmacological interventions, however, have limited efficacy and severe-side effects for patients; therefore, we are in need of new, effective, and safe alternative treatment options.

Recently, deep brain stimulation (DBS) has shown to have beneficial effects across memory and cognitive networks. A first evidence for this emerged when Hamani and colleagues stimulated the fornix/hypothalamus area in a patient suffering from morbid obesity [5]. In this specific case, DBS generated detailed autobiographical memories in the patient. Based on this case-observation, the same group performed a phase-I trial in patients with Alzheimer's Disease (AD) [6]. Indeed, memory tests confirmed possible improvements and/or slowing in the rate of cognitive decline in some patients following chronic DBS of 12 months.

Despite the encouraging results of the clinical trials presented above, basic neural and chemical mechanisms underlying DBS are still debated [7]. One approach to address these issues is to investigate the effects by stimulating homologous regions in experimental animal models [8].

Therefore, the studies described in this dissertation aimed at investigating which DBS target structures and stimulation parameters produce the most beneficial effects in an 
experimental model of dementia. In addition, the present dissertation also describes potential mechanisms of action of DBS with regard to memory restoration. Only through understanding the mechanisms, DBS therapy in dementia patients can be fine-tuned to produce the best possible symptom relief currently available.

\section{TARGET GROUPS}

Target groups of the research presented in this dissertation are broad.

The first target audience entails the patients and their caregivers. As described above, effective dementia treatment represents a major unmet medical need at present. The quality of life of millions of patients as well as their caregivers significantly decreases in the progression of the disease. The consequences usually include a substantial decline in family's health and emotional well-being. Unfortunately, drug-based research activities in the last years have not resulted in breakthroughs in treating patients with dementia. In this respect, DBS might open new ways for alternative, non-drug based treatment in dementia-related disorders. In line with this, by examining the effects of DBS in an experimental model of dementia, we might have established the groundwork of new treatment options for not only for patients with dementia, but also for patients suffering from other cognitive impairing CNS diseases. In fact, many neurological and psychiatric conditions are constituted by cognitive disorders, for example patients with schizophrenia, attention-deficit hyperactivity disorder, depression and addiction often suffer from comorbid cognitive impairment. Thus, the present dissertation provides primary findings, which help to elucidate how DBS could potentially improve cognitive functions in dementia and other neurological and psychiatric diseases.

Coherent to this, the general public forms another target audience. Dementia has been defined as the most costly disease of our time. Treatment costs are covered by health insurances, affected individuals, their families and private insurances. In this respect, alleviating symptoms of dementia by means of DBS, would not only improve the quality of life of patients and caregivers, it would also provide a major financial relief for health care costs. Thus, delaying institutionalisation of patients by means of DBS can have an impact on economy and society, because patients function independently for longer, i.e. they can work, consume and invest for longer.

The third target group comprises the scientific critical mass. The findings of which brain region target for DBS produces most beneficial effects with regard to memory restoration might be of interest to neurosurgeons. Out of all structures within the circuit of Papez, the studies described in this dissertation, and other studies indicate that the fornix is the most relevant one for dementia therapy $[9,10]$. Finally, the academic community as well as neuromodulation companies might profit from findings within 
this dissertation. For example, there is an increasing awareness for the necessity to develop adaptive DBS systems (i.e., closed-loop), which prevent side-effects caused by overstimulation. Closed-loop feedback systems are latest advances in DBS therapy for epilepsy, but have not yet been developed for AD.

\section{ACTIVITY/PRODUCTS}

The major product/finding which can be derived by studies in this dissertation is that DBS might constitute an alternative treatment option for patients with dementia-related disorders. Results of the current dissertation provide evidence, that the fornix is the most suitable DBS target structure within the circuit of Papez to restore memory loss in an experimental rat model of dementia. The effects of fornix DBS are accompanied by selective neural activation in the hippocampus as well as increased hippocampal acetylcholine levels and no long-term neurogenic changes. In fact, the experiments in Chapter 9 have indicated that adaptive mechanism-based DBS might be necessary to maintain beneficial memory effects.

Chapter 3 of this dissertation also sheds light on the fact that up to now, most DBS studies in psychiatric disorders were first conducted in humans. Since the clinical data and the findings from animal studies show similarities, animal models can be of important value to find potential new DBS targets and settings for memory restoration. Close collaboration between basic scientists and clinicians has led to a successful implementation of preclinical findings to clinical DBS over the past years. For a successful treatment, such as DBS in patients with Parkinson's disease, a solid scientific base is needed. Increased understanding of basal ganglia function from studies in translational Parkinson's disease models $[11,12]$ paved the way for the clinical application of this method [13] and became the most successful application of DBS thus far.

\section{INNOVATION}

The studies of this dissertation have been innovative for several reasons. This work comprised in-depth investigation of the recently introduced concept of treating demented patients with DBS. This is a novel concept that might provide more rapid and robust therapies for patients with dementia. Furthermore, a multi-level, interdisciplinary approach was applied: bilateral DBS in freely moving animals, behavioral assessment, microdialysis and histology. This multi-level approach helped to understand if and how memory can be improved by DBS, in particular what the potential mechanisms 
of action are. Novel mechanisms of DBS were identified, which might facilitate the treatment and management of patients with dementia in the future.

\section{IMPLEMENTATION}

In line with the abovementioned relevance of this project for patients, society and the scientific community, the implementation of the knowledge generated by this dissertation is again at various levels. Therefore, novel insights will be shared with patient organizations, health care professionals and scientific societies. From an academic perspective, results have been or will be published in peer-reviewed international journals and are presented at national and international conferences. 


\section{REFERENCES}

1. Policy Brief for Heads of Government: The Global Impact of Dementia 2013-2050. London: Alzheimer's Disease International; 2013.

2. Batsch N, Mittelman M. World Alzheimer Report 2012: Overcoming the stigma of dementia. 2012.

3. Wimo A, Prince M. World Alzheimer Report 2010: The global economic impact of dementia. London: 2010 .

4. Thies W, Bleiler L. 2011 Alzheimer's disease facts and figures. Alzheimers Dement. 2011;7(2):208-44. Epub 2011/03/19. eng.

5. Hamani C, McAndrews MP, Cohn M, Oh M, Zumsteg D, Shapiro CM, et al. Memory enhancement induced by hypothalamic/fornix deep brain stimulation. Annals of Neurology. 2008;63(1):119-23.

6. Laxton AW, Tang-Wai DF, McAndrews MP, Zumsteg D, Wennberg R, Keren R, et al. A phase I trial of deep brain stimulation of memory circuits in Alzheimer's disease. Annals of Neurology. 2010;68(4):521-34.

7. Kringelbach ML, Green AL, Owen SL, Schweder PM, Aziz TZ. Sing the mind electric - principles of deep brain stimulation. Eur J Neurosci. 2010;32(7):1070-9.

8. Hamani C, Temel Y. Deep Brain Stimulation for Psychiatric Disease: Contributions and Validity of Animal Models. Sci Transl Med. 2012;4(142):142rv8.

9. Hescham S, Jahanshahi A, Meriaux C, Lim LW, Blokland A, Temel Y. Behavioral effects of deep brain stimulation of different areas of the Papez circuit on memory- and anxiety-related functions. Behav Brain Res. 2015;292:353-60.

10. Hescham S, Lim LW, Jahanshahi A, Steinbusch HW, Prickaerts J, Blokland A, et al. Deep brain stimulation of the forniceal area enhances memory functions in experimental dementia: the role of stimulation parameters. Brain Stimul. 2013;6(1):72-7. Epub 2012/03/13. eng.

11. Bergman H, Wichmann T, DeLong MR. Reversal of experimental parkinsonism by lesions of the subthalamic nucleus. Science. 1990;249(4975):1436-8.

12. Benazzouz A, Gross C, Feger J, Boraud T, Bioulac B. Reversal of rigidity and improvement in motor performance by subthalamic high-frequency stimulation in MPTP-treated monkeys. The European journal of neuroscience. 1993;5(4):382-9.

13. Pollak P, Benabid AL, Gross C, Gao DM, Laurent A, Benazzouz A, et al. [Effects of the stimulation of the subthalamic nucleus in Parkinson disease]. Revue neurologique. 1993;149(3):175-6. Effets de la stimulation du noyau sous-thalamique dans la maladie de Parkinson. 



\section{ACKNOWLEDGEMENTS}

Completing a $\mathrm{PhD}$ is undeniably a long and arduous process, which cannot be accomplished alone. I have been blessed with the best network of supervisors, co-supervisors, advisors, colleagues, friends and family who have supported me throughout my studies. I would like to take the opportunity and express my sincere gratitude to everybody, who has made it possible for me to complete my $\mathrm{PhD}$.

First and foremost, I consider myself immensely fortunate to have worked under the supervision of Prof. Yasin Temel. Dear Yasin, thank you for giving me the opportunity to work on this challenging topic as a $\mathrm{PhD}$ student. You have not only put unwavering faith in my abilities over the years, but also allowed me to blossom under your supervision. Your sustained guidance, support and trust have been invaluable and greatly appreciated.

My profound gratitude also extends to my first co-supervisor Dr. Arjan Blokland for his time, energy and constructive feedback throughout the entire process of my $\mathrm{PhD}$ work. Dear Arjan, you have taught me how to design well-controlled experiments and inspired me with your enthusiasm for research, thank you.

I would like to give a heartfelt and special thanks to my second co-supervisor Dr. Ali Jahanshahi for his most appreciated advice and friendly help with so many designrelated and technical aspects of my study. Thank you for your genuine caring and faith in me during the dissertation process.

Similar, heartfelt gratitude goes to Prof. Trevor Sharp, who has generously hosted me in his laboratory in Oxford. Thank you for your valuable guidance and support throughout my research stay, I have very fond memories of my time in England.

I would also like to thank Dr. LeeWei Lim. Dear Anthony, thank you for your sustained guidance, patience and help in different aspects of this $\mathrm{PhD}$ project. I am especially indebted to you for your assistance in the beginning phase of my $\mathrm{PhD}$.

I take this opportunity to express my gratitude to all of the Department faculty members:

Prof. Harry Steinbusch, thank you for your constructive feedback on various occasions during my $\mathrm{PhD}$. Your advice has both challenged and encouraged me in my studies and your passion for science has been contagious. Because of your excellent achievements as director of our school, I can look back at a productive and inspiring time at Maastricht University.

Sincere thanks also to Dr. Bart Rutten for your encouragement and guidance throughout my work for this thesis. As division leader, you have created a friendly and stimulating working atmosphere in our department. Thank you. 
Dr. Govert Hoogland, thank you for your constant help during these years. Whether we discussed papers together or brainstormed about upcoming experiments, I always appreciated your friendly advice.

Same amounts to Dr. Jos Prickaerts. I learned a lot from you with regard to cognitive neuroscience as well as how to perform various behavioural experiments. Thank you also for being part of the opposition during my defense.

Prof. Marc de Baets, Dr. Mario Losen, Dr. Pilar Martinez. I always appreciated the intellectually stimulating working environment in our department as well the many expert group meetings and social events we had together. Thank you.

I would also like to thank the assessment committee and opposition for their time to critically evaluate my thesis. Thank you, Prof. Koo van Overbeeke for being the chairman during my defense. Dr. Daniel van den Hove, Prof. Frans Verhey, Dr. Clement Hamani and Prof. Jens Kuhn, thank you for your time and effort to critically review my thesis. It goes without saying, that my promotion would not have been possible without your valuable input. Thank you very much!

I am especially grateful to Hellen Steinbusch, Marjan Philippens and Denise Hermes for their engagement, interest and most appreciated help during my experiments. The whole department is immensely fortunate to have you in the lab.

My sincere gratitude is also owed to Marie-Thérèse Moers-Kockelmans, Rachelle Capponi, Nicole Senden and Peggy Bisschoff for their wonderful secretary skills, which includes organizing brilliant meetings and events for our department. I would also like to thank Ankie Hochstenbach and Laurent Louwies for their immaculate financial and administrative coordination.

At this stage, I would like to thank my dear fellow PhD students (whether from the past or present) from the whole neuroscience department for their encouragement and support throughout my $\mathrm{PhD}$ period. I always appreciated the nice, interdisciplinary working atmosphere. It was a real pleasure to work with all of you and you all contributed in some way to this thesis. Some of you, I would like to thank explicitly.

Jo Stevens, thank you for your valuable support with the DSU microscope and helping me with DMIII- related issues. Jochen de Vry, thank you for your interest and help with various immunohistochemical aspects of my study. Anna Schueth, you were not only the first person I met in Maastricht, but also a great help in the beginning phase of my $\mathrm{PhD}$, thank you.

During my research stay in Oxford, I received most appreciated support from Julia Brouard, Ayesha Sengupta, Katharina Stumpenhorst, Chris Barker and Florence Serres. Special thanks to Judith Schweimer for your patience and help during this period.

In team Temel, I had the privilege to work with ambitious, motivated and bright colleagues. A PhD journey is easier when you travel together. In this respect, I would like 
to thank Birgit Plantinga, Jasper Smit, Gusta van Zwieten, Maartje Melse, Youssef Yakkioui, Remco Santegoeds, Mark Janssen, Sonny Tan, Rinske Vlamings, Majed Aldehri, Paul Janssen, Fred Schaper and Anne Mulders for their constant encouragements throughout my $\mathrm{PhD}$.

Joao Casaca Carreira, Sandra Schipper and Melanie Lagiere, thank you for all your help and advice throughout these last years. Your special skill to spread happiness on those scientifically dark days, has made it possible to overcome all difficulties I encountered. Thank you for your encouragement, care and understanding.

Finally, my paranymphs Lisa Schönfeld and Céline Meriaux, who have been there for me for almost the entire process of my PhD. Thank you so much for your time, help and organization of my defense and especially for keeping my nerves calm. I am very fortunate to have your support on this important day and wanted to thank you for everything. Here is to good times, good memories and many more to come.

Finally, this work would not have been possible without many people behind the scenes of the lab.

First, I would like to thank my family in the U.S., Pam, Edgar, Meagen and Tristen Medellin. I made the decision to study B.Sc. Neuroscience while I was visiting you in 2006 and although I doubted initially, you believed in me from the start. Thank you for your constant encouragement. I would not be, where I am today without your support.

I also owe a big thank you to Carmen Roemer, Kathrin Rütten and the whole family. Dear Carmen, thank you for your support as well as the well-deserved distractions during my years as a PhD student. With every new challenge I could always count on you. Shared trouble is half the trouble, shared joy is twice the joy.

Leni Kurstjens, Nicoll, Ans and Sjaak Kierkels, thank you for your encouragement and help throughout the years.

Christian Schmidt, Florian Birkenhauer and Alfred Wittek had to listen to my labstories on various family occasions. Thank you very much for your patience.

On a more personal note, I will write the remaining parts in Polish and German.

Dla Ewy, Roberta, Łukasza i Karola Chlebickich: Nawet jeśli się tak często nie widzimy, to wiem że zawsze mogę na Was liczyć. Dziękuję wam bardzo. To jest ważne, gdy ktoś myśli,pamięta i wspiera.

Dla Beaty, Janusza i Patryka Lawickich: Jeszcze raz dziękuję Wam za pomoc przy wykończeniu domu. Dzięki Waszej pomocy zyskałam dużo czasu który był dla mie niezbędnie na koncentrowaniu się nad pracą doktorancką.

Dla Maryli i Ryszarda Jechurów: Jako moi chrzestni zawsze we mnie wierzyliście. Dziękuję wam bardzo.

Lieber Erik, du warst von Anfang an dabei und hast besonders viel zu dieser Arbeit beigetragen. In schwierigen Situationen hast du immer an mich geglaubt und mir mit Rat und Tat zur Seite gestanden. Ob du nun mit mir im Labor warst, meine Präsenta- 
tionen angehört oder Paper durchgelesen hast, nichts war zu viel gefragt. Vielen Dank für alles!

Zu guter Letzt, vielen Dank Mama, Eva und Miriam. Ihr wart immer für mich da, auch wenn ich mich entschloss, mal wieder mit dem Kopf durch die Wand zu gehen. „Zusammen sind wir stark.“ 


\section{BIOGRAPHY}

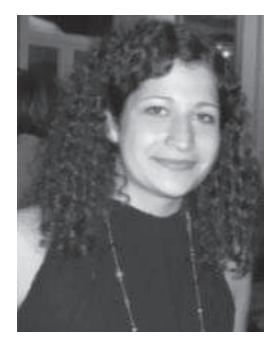

Sarah-Anna Hescham was born on 8 July 1987 in Tönisvorst, Germany. After graduating from the Albertus-Magnus Gymnasium, Viersen in 2006, she went to study Neuroscience at the University of Cologne, Germany. She completed her practical semester and Bachelor's project at the Neuroscience Department, University of Cape Town, South Africa, under the supervision of Prof. Vivienne Russell. This research stay resulted in 2 publications in international peer-reviewed journals and Sarah received her B.Sc. degree in 2009. Intrigued by Neuroscience, Sarah decided to continue her studies at the University of Göttingen, Germany, where she pursued a M.Sc. degree in Neuroscience in 2011. For her Master's project, she worked at the Centre of Clinical Research in Neuropsychiatry, Perth, Western Australia under the supervision of Prof. Mathew Martin-Iverson. Following this, she started her doctoral research at the School for Mental Health and Neuroscience at Maastricht University, The Netherlands, supervised by Prof. Yasin Temel. As a PhD student, Sarah spent 7 months in the laboratory of Prof. Trevor Sharp from the Pharmacology Department of Oxford University, U.K. The presented work in this thesis received various awards for best poster or best publication at scientific meetings. In June 2015, Sarah has been awarded the Kootstra Talent Fellowship of Maastricht University to continue her scientific career in the arena of cognitive neuroscience. 



\section{THESIS DEFENSES FROM MHENS - SCHOOL FOR MENTAL HEALTH AND NEUROSCIENCE}

2013

Rob Havermans: Bipolar disorder in daily life; Mood and cortisol responses to naturally occurring events. Supervisor: Prof.dr. M. de Vries; Co-Supervisor: Dr. N. Nicolson.

Véronique Moers-Hornikx: Deep brain stimulation and the cerebellum. Supervisors: Prof.dr. J. Vles / Prof. dr. Y. Temel; Co-Supervisor: Dr. G. Hoogland.

Nicole Veldhorst-Janssen: Intranasal delivery of rapid acting drugs. Supervisors: Prof.dr. M. Marcus / Prof. dr. C. Neef; Co-Supervisor: Dr. P.H. van der Kuy.

Stéphanie Knippenberg: Vitamin D and Multiple Sclerosis: immunological and clinical outcome. Supervisor: Prof.dr. J. Cohen-Tervaert; Co-Supervisors: Dr. J. Damoiseaux / Dr. Y. Bols.

Erik D. Gommer: Dynamic Cerebral Autoregulation: from methodology towards clinical application. Supervisors: Prof.dr. W.H. Mess / Prof.dr. R.B. Panerai, UK; Co-Supervisor: Dr.ir. J.P.H. Reulen.

Olga A.H. Reneerkens: Can PDE inhibition improve cognition ? Translational insights. Supervisor: Prof. dr. H.W.M. Steinbusch; Co-Supervisor: Dr. J. Prickaerts

Lyzel S. Elias-Sonnenschein: Clinical and biomarker correlates of genetic risk factors for Alzheimer's disease. Supervisor: Prof.dr. F.R.J. Verhey; Co-Supervisor: Dr. P.J. Visser.

Diego F. Mastroeni: Epigenetic Dysregulation and the Pathophysiology of Alzheimer's Disease. Supervisors: Prof.dr. H.W.M. Steinbusch / Prof.dr. P.D. Coleman, Sun City, Arizona; Co-Supervisors: Dr. B.P.F. Rutten / Dr. D.L.A. van den Hove.

Leonidas Chouliaras: Epigenetic Regulation in Aging and Alzheimer's disease: A translational perspective. Supervisor: Prof.dr. H.W.M. Steinbusch; Co-Supervisors: Dr. B.P.F. Rutten / Dr. D.L.A. van den Hove.

Liesbeth Knaepen: Perinatal events and altered pain sensitivity in later life. Supervisors: Prof.dr. E.A.J. Joosten / Prof.dr. D. Tibboel, EUR; Co-Supervisor: Dr. J. Patijn.

Marisela Martinez-Claros: Hippocampal plasticity and corticosterone: From dendrites to behaviour. Supervisor: Prof.dr. H.W.M. Steinbusch; Co-Supervisors: Dr. J.L. Pawluski / Dr. J. Prickaerts.

Marcus D. Lancé: A circle of improvement in bleeding management: from laboratory to clinic and back. Supervisors: Prof.dr. M.A.E. Marcu / Prof.dr. J.W.M. Heemskerk; Co-Supervisor: Dr. Y.M.C. Henskens.

Hilde Braakman: Imaging the brain; neuronal correlates of cognitive impairment in children with frontal lobe epilepsy. Supervisors: Prof.dr. A.P. Aldenkamp / Prof.dr. J.S.H. Vles; Co-Supervisors: Dr.ir. W.H. Backes / Dr. P.A.M. Hofman.

Willem H. van Zwam: Aneurysmal subarachnoid hemorrhage: imaging strategies and cost-effectiveness aspects in diagnostic work-up and post-therapeutic follow-up. Supervisors: Prof.dr. J.T. Wilmink / Prof.dr. J.E. Wildberger; Co-Supervisor: Dr. P.A.M. Hofman.

Klara De Cort: The Pathogenesis of Panic Disorder. Supervisors: Prof.dr. I. Myin-Germeys / Prof.dr. E.J.L. Griez; Co-Supervisors: Dr. K.R.J. Schruers / Dr. I. Van Diest, Leuven.

Kim van Wijck: Mind the Gap; experimental studies on splanchnic hyperfusion and gastrointestinal integrity loss in man. Supervisors: Prof.dr. W.A. Buurman / Prof.dr. C.H.C. Dejong; Co-Supervisor: Dr. K. Lenaerts.

Yvette Roke: Antipsychotic-induced hyperprolactinemia in children and adolescents with mainly autism spectrum disorders. Prevalence, symptoms, clinical consequences and genetic risk factors. 
Supervisors: Prof.dr. P.N. van Harten / Prof.dr. J.K. Buitelaar (RUN); Co-Supervisor: Dr. A. Boot (UMCG).

Fleur Goezinne: Retinal detachment surgery: pre and postoperative prognostic factors. Supervisors: Prof. dr. F. Hendrikse / Prof.dr. C.A.B. Webers; Co-Supervisor: Dr. E.C. La Heij (Amsterdam).

Ralph L.J.G. Maassen: The Merits of Videolaryngoscopy during Glottic Visualisation for Endotracheal

Intubation. Supervisors: Prof.dr. M. Marcus / Prof.dr. A. van Zundert (University of Queensland).

Maria J. de Sousa Guerreiro: The role of sensory modality in age-related distraction. Supervisor: Prof.dr. C.M. van Heugten; Co-Supervisor: Dr. P.W.M. van Gerven.

Ine Rayen: Effects of developmental fluoxetine exposure on neurobehavioral outcomes. Supervisor: Prof. dr. H.W.M. Steinbusch; Co-Supervisors: Dr. J.L. Pawluski / Dr. T.D. Charlier (Ohio University, USA).

Nynke M.G. Bodde: Psychogenic non-epileptic seizures; a separate disorder or part of a continuum? Supervisors: Prof.dr. R. van Oostenbrugge / Prof.dr. K. Vonck (UZ Gent);

Co-Supervisors: Dr. R. Lazeron / Dr. A. de Louw (Epilepsiecentrum Kempenhaeghe, Heeze).

Alejandro M. Gomez: Novel strategies for making myasthenia less gravis: targeting plasma cells and the neuromuscular junction. Supervisor: Prof.dr. M.H. De Baets; Co-Supervisors: Dr. M. Losen / Dr. P. Martinez-Martinez.

Mohammad S. Rahnama'i: Prostaglandins and Phosphodiesterases in the Urinary Bladder Wall. Supervisors: Prof.dr. Ph. Van Kerrebroeck / Prof.dr. S. de Wachter (Universiteit Antwerpen); Co-Supervisor: Dr. G. van Koeveringe.

Mariken B. de Koning: Studying biomarkers in populations at genetic and clinical high risk for psychosis. Supervisors: Prof.dr. T. Amelsvoort / Prof.dr. J. Booij (AMC).

Fabien Boulle: Epigenetic regulation of BDNF/TrkB signaling in the pathophysiology and treatment of mood disorders. Supervisors: Prof.dr. H.W.M. Steinbusch / Prof.dr. L. Lanfumey (Universiteit Parijs); Co-Supervisors: Dr. D. van den Hove / Dr. G. Kenis.

2014

Iris Nowak-Maes: Tinnitus; assessment of quality of life \& cost-effectiveness. Supervisors: Prof.dr. M. Peters / Prof.dr. B. Kremer; Co-Supervisors: Dr. M. Joore / Dr. L. Anteunis.

Marjolein Huijts: Cognitive function in patients with cerebral small vessel disease. Supervisor: Prof.dr. R.J. van Oostenbrugge; Co-Supervisors: Dr. A.A. Duits / Dr. J. Staals.

Markus Gantert: Fetal inflammatory injury as origin of long term disease: Lessons from animal models. Supervisors: Prof.dr. B. Kramer / Prof.dr. L. Zimmermann; Co-Supervisor: Dr. A. Gavilanes.

Elke Kuypers: Fetal development after antenatal exposures: Chorioamnionitis and maternal glucocorticoids. Supervisors: Prof.dr. B.W. Kramer / Prof.dr. H.W. Steinbusch / Prof.dr. Suhas G. Kallapur (University of Cincinnati, Ohio, USA).

Pieter Kubben: Ultra low-field strength intraoperative MRI for Glioblastoma Surgery. Supervisor: Prof.dr. J.J. van Overbeeke; Co-Supervisor: Dr. H. van Santbrink.

Laura Baijens: Surface electrical stimulation of the neck for oropharyngeal dysphagia in Parkinson's disease: therapeutic aspects and reliability of measurement. Supervisor: Prof.dr. B. Kremer; CoSupervisor: Dr. R. Speyer, Townsville.

Janneke Hoeijmakers: Small fiber neuropathy and sodium channels; a paradigm shift. Supervisor: Prof.dr. R.J. van Oostenbrugge; Co-Supervisors: Dr. C.G. Faber / Dr. I.S.J. Merkies.

Stephanie Vos: The Role of biomarkers in preclinical and prodromal Alzheimer's disease. Supervisor: Prof.dr. F.R. Verhey; Co-Supervisor: Dr. P.J. Visser.

Muriël Doors: The Value of Optical Coherence Tomography in Anterior Segment Surgery. Supervisors: Prof.dr. R.M. Nuijts / Prof.dr. C.A. Webers; Co-Supervisor: Dr. T.T.J.M. Berendschot. 
Anneke Maas: Sleep problems in individuals with genetic disorders associated with intellectual disability. Supervisors: Prof.dr. I. Curfs / Prof.dr. R. Didden.

Sebastiaan van Gorp: Translational research on spinal cord injury and cell-based therapies; a focus on pain and sensorimotor disturbances. Supervisors: Prof.dr. B. Joosten / Prof.dr. M. van Kleef; CoSupervisors: Dr. J. Patijn /Dr. R. Deumens, KU Leuven.

Andrea Sannia: High risk newborns and brain biochemical monitoring. Supervisor: Prof.dr. J.S.H. Vles; Co-Supervisors: Dr. D. Gazzolo, Alessandria, Italy / Dr. A.W.D. Gavilanes.

Julie A.D.A. Dela Cruz: Dopamine mechanisms in learning and memory: Evidence from rodent studies. Supervisors: Prof.dr. H.W.M. Steinbusch / Prof.dr. R.J. Bodnar, New York; Co-Supervisor: Dr. B.P.F. Rutten.

René Besseling: Brain wiring and neuronal dynamics; advances in MR imaging of focal epilepsy. Supervisors: Prof.dr. A.P. Aldenkamp / Prof.dr.ir. W.H. Backes; Co-Supervisor: Dr. J.F.A. Jansen.

Maria Quint-Fens: Long-term care after stroke; development and evaluation of a long-term intervention in primary care. Supervisors: Prof.dr. J.F.M. Metsemakers / Prof.dr. C.M. van Heugten / Prof.dr. M. Limburg, Almere; Co-Supervisor: dr. G.H.M.I. Beusmans.

Veronique Moulaert: Life after survival of a cardiac arrest; the heart of the matter. Supervisors: Prof.dr. J.A. Verbunt / Prof.dr. C.M. van Heugten / Prof.dr. D.T. Wade, Oxford, UK.

Feikje Smeets: The hallucinatory-delusional state: a crucial connection in the psychosis symptom network. Supervisor: Prof.dr. J. van Os; Co-Supervisor: Dr. T. Lataster.

Lies Clerx: Alzheimer's disease through the MR-eye; novel diagnostic markers and the road to clinical implementation". Supervisor: Prof.dr. F. Verhey; Co-Supervisors: Dr. P.J. Visser / P. Aalten.

Sonny Tan: The subthalamic nucleus in Parkinson's disease. Supervisors: Prof.dr. Y. Temel / Prof.dr. H.W.M. Steinbusch / Prof.dr. T. Sharp, Oxford, UK / Prof.dr. V. Visser-Vandewalle, Koln.

Koen van Boxem: The use of pulsed radiofrequency in the management of chronic lumbosacral radicular pain. Supervisors: Prof.dr. M. van Kleef / Prof.dr. E.A.J. Joosten; Co-Supervisor: Assoc. Prof.dr. J. van Zundert.

Jérôme Waterval: Hyperostosis cranialis interna. Supervisors: Prof.dr. J.J. Manni / Prof.dr. R.J. Stokroos.

Sylvie Kolfschoten-van der Kruijs: Psychogenic non-epileptic seizures; the identification of neurophysiological correlates. Supervisors: Prof.dr. A.P. Aldenkamp / Prof.dr. K.E.J. Vonck, Universiteit Gent; Co-Supervisors: Dr. J.F.A. Jansen / Dr. R.H.C. Lazeron, Kempenhaeghe.

Wouter Pluijms: Spinal cord stimulation and pain relief in painful diabetic: polyneuropathy, a translational approach. Supervisors: Prof.dr. M. van Kleef /

Prof.dr. E.A. Joosten; Co-supervisor: Dr. C.G. Faber.

Ron Handels: Health technology assessment of diagnostic strategies for Alzheimer's disease. Supervisors: Prof.dr. F.R.J. Verhey / Prof.dr. J.L. Severens (EUR); Co-Supervisor: Dr. M.A. Joore / Dr. C.A.G. Wolfs.

Evelyn Peelen: Regulatory T cells in the pathogenesis of Multiple Sclerosis: potential targets for vitamin D therapy. Supervisors: Prof.dr. R.M.M. Hupperts / Prof.dr. J.W. Cohen Tervaert; Co-Supervisor: Dr. J.G.M.C. Damoiseaux / Dr. M.M.G.L.Thewissen, Diepenbeek.

Reint Jellema: Cell-based therapy for hypoxic-ischemic injury in the preterm brain. Supervisors: Prof.dr. B.W.W. Kramer / Prof.dr. H.W.M. Steinbusch; Co-Supervisor: Dr. W.T.V. Germeraad / Dr. P. Andriessen, Veldhoven.

Maria Wertli: Prognosis of Chronic Clinical Pain Conditions: The Example of Complex Regional Pain Syndrome 1 and Low Back Pain. Supervisors: Prof.dr. M. van Kleef; Co-Supervisor: Dr. F. Brunner, Zürich / Dr. R. Perez, VUmc. 
Dagmar Zeef: An experimental model of Huntington's disease: Validation \& Stimulation. Supervisors: Prof.dr. Y. Temel / Prof.dr. H.W.M. Steinbusch; Co-supervisor:

Dr. A. Jahanshahi.

Jeroen Decoster: Breaking Down Schizophrenia into phenes, genes and environment. Supervisors: Prof. dr. I. Myin-Germeys / Prof.dr. M. De Hert, KU Leuven; Co-Supervisor: Dr. R. van Winkel.

Eaja Anindya Sekhar Mukherjee: Fetal Alcohol Spectrum Disorders: exploring prevention and management. Supervisor: Prof.dr. L.M.G. Curfs; Co-Supervisor: Prof. S. Hollins, St. George’s University of London, UK.

Catherine van Zelst: Inside out; On stereotype awareness, childhood trauma and stigma in psychosis. Supervisors: Prof.dr. Ph. Delespaul / Prof.dr. J. van Os.

Ibrahim Tolga Binbay: Extended Psychosis Phenotype in the Wider Social Environment. Supervisor: Prof. dr. J. van Os; Co-Supervisor: Dr. M. Drukker.

Frank Van Dael: OCD matters in psychosis. Supervisors: Prof.dr. J. van Os / Prof.dr. I. Myin-Germeys.

Pamela Kleikers: NOXious oxidative stress: from head toe too and back. Supervisors: Prof.dr. H.H.H.W. Schmidt / Prof.dr. H.W.M. Steinbusch; Co-Supervisor: Dr. B. Janssen.

José Luis Gerardo Nava: In vitro assay systems in the development of therapeutic interventions strategies for neuroprotection and repair. Supervisors: Prof.dr.med. J. Weis / Prof.dr. H.W.M. Steinbusch; Co-Supervisor: Dr. G.A. Brook, RWTH Aachen.

Eva Bollen: Cyclic nucleotide signaling and plasticity. Supervisors: Prof.dr. H.W.M. Steinbusch / Prof.dr. R. D’Hooge, KU Leuven; Co-Supervisor: Dr. J. Prickaerts.

2015

Jessica A. Hartmann: A good laugh and a long sleep; Insights from prospective and ambulatory assessments about the importance of positive affect and sleep in mental health. Supervisor: Prof.dr. J. van Os; Co-Supervisors: C.J.P. Simons / Dr. M. Wichers.

Bart Ament: Frailty in old age; conceptualization and care innovations. Supervisors: Prof.dr. G.I.J.M. Kempen / Prof.dr. F.R.J. Verhey; Co-Supervisor: Dr. M.E. de Vugt.

Mayke Janssens: Exploring course and outcome across the psychosis-continuum. Supervisor: Prof.dr. I. Myin-Germeys; Co-Supervisor: Dr. T. Lataster.

Dennis M.J. Hernau: Dopayours is not dopamine: genetic, environmental and pathological variations in dopaminergic stress processing. Supervisor: Prof.dr. I. Myin-Germeys; Co-Supervisors: Prof.dr. F.M. Mottaghy / Dr. D. Collip.

Ingrid M.H. Brands: The adaptation process after acquired brain injury Pieces of the puzzle. Supervisors: Prof.dr. C.M. van Heugten / Prof.dr. D.T. Wade, Oxford UK;

Co-Supervisors: Dr. S.Z. Stapert / Dr. S. Köhler.

Francesco Risso: Urinary and salivary S100B monitoring in high risk infants. Supervisor: Prof.dr. J.S.H. Vles; Co-Supervisors: Dr. D. Gazzolo, Genoa,Italy / Dr. A.W.D. Gavilanes.

Alessandro Borghesi: Stem and Progenitor Cells in Preterm Infants: Role in the Pathogenesis and Potential for Therapy. Supervisor: Prof.dr. L. Zimmermann; Prof.dr. B. Kramer; Co-Supervisors: Dr. D. Gazzolo, Genoa,Italy / Dr. A.W.D. Gavilanes.

Claudia Menne-Lothmann: Affect dynamics; A focus on genes, stress, and an opportunity for change. Supervisor: Prof.dr. J. van Os; Co-Supervisors: Dr. M. Wichers / Dr. N. Jacobs

Martine van Nierop: Surviving childhood new perspectives on the link between childhood trauma and psychosis. Supervisors: Prof.dr. I. Myin-Germeys / Prof.dr. J. van Os; Co-Supervisor: Dr. R. van Winkel. 
Sylvia Klinkenberg: VNS in children; more than just seizure reduction. Supervisors: Prof.dr. J. Vles / Prof. dr. A. Aldenkamp; Co-Supervisor: Dr. H. Majoie.

Anouk Linssen: Considerations in designing an adult hearing screening programme. Supervisor: Prof.dr. B. Kremer; Co-Supervisors: Dr. L. Anteunis / Dr. M. Joore.

Janny Hof: Hearing loss in young children; challenges in assessment and intervention. Supervisors: Prof. dr. B. Kremer / Prof.dr. R. Stokroos / Prof.dr. P. van Dijk, RUG; Co-Supervisor: Dr. L. Antheunis.

Kimberly Cox-Limpens: Mechanisms of endogenous brain protection; Clues from the transcriptome. Supervisors: Prof.dr. J. Vles / Prof.dr. L. Zimmermann; Co-Supervisor: Dr. A. Gavilanes.

Els Vanhoutte: Peripheral Neuropathy outcome measures; Standardisation (PeriNomS) study part 2: Getting consensus. Supervisors: Prof.dr. C. Faber / Prof.dr. P. van Doorn; Co-Supervisor: Dr. I. Merkies, Spaarne ziekenhuis Hoofddorp.

Mayienne Bakkers: Small fibers, big troubles; diagnosis and implications of small fiber neuropathy. Supervisors: Prof.dr. C. Faber / Prof.dr. M. de Baets; Co-Supervisor: Dr. I. Merkies, Spaarne ziekenhuis Hoofddorp.

Ingrid Kramer: Zooming into the micro-level of experience: An approach for understanding and treating psychopathology. Supervisor: Prof.dr. J. van Os; Co-Supervisors: Dr. M. Wichers, UMC Groningen / Dr. C. Simons.

Esther Bouman: Risks and Benefits of Regional Anesthesia in the Perioperative Setting. Supervisors: Prof.dr. M. van Kleef / Prof.dr. M. Marcus, HMC, Qatar / Prof.dr. E. Joosten; Co-Supervisor: Dr. H. Gramke.

Mark Janssen: Selective stimulation of the subthalamic nucleus in Parkinson's disease; dream or near future. Supervisors: Prof.dr. Y. Temel / Prof.dr. V. Visser-Vandewalle, Keulen / Prof.dr. A. Benazzouz, Bordeax, France.

Reina de Kinderen: Health Technology Assessment in Epilepsy; economic evaluations and preference studies. Supervisors: Prof.dr. S. Evers / Prof.dr. A. Aldenkamp; Co-Supervisor: Dr. H. Majoie / Dr. D. Postulart, GGZ O-Brabant.

Saskia Ebus: Interictal epileptiform activity as a marker for clinical outcome. Supervisors: Prof.dr. A. Aldenkamp / Prof.dr. J. Arends, TUE / Prof.dr. P. Boon, Universiteit Gent, België.

Inge Knuts: Experimental and clinical studies into determinants of panic severity. Supervisor: Prof.dr. I. Myin-Germeys; Co-Supervisor: Dr. K. Schruers

Nienke Tielemans: Proactive coping post stroke: The Restored4Stroke Self-Management study. Supervisors: Prof.dr. C. van Heugten / Prof.dr. J. Visser-Meily, UMC Utrecht; Co-Supervisor: Dr. V. Schepers, UMC Utrecht.

Tom van Zundert: Improvements Towards Safer Extraglottic Airway Devices. Supervisors: Prof.dr. A.E.M. Marcus / Prof.dr. W. Buhre / Prof.dr. J.R. Brimacombe, Queensland, Australia / Prof.dr. C.A. Hagberg.

Tijmen van Assen: Anterior Cutaneous Nerve Entrapment Syndrome Epidemiology and surgical management. Supervisors: Prof.dr. G.L. Beets / Prof.dr. M. van Kleef / Dr. R.M.H. Roumen / Dr. M.R.M. Scheltinga, MMC Veldhoven.

Rohit Shetty: Understanding the Clinical, Immunological and Genetic Molecular Mechanisms of Keratoconus. Supervisors: Prof.dr. R.M.M.A. Nuijts / Prof.dr. C.A.B. Webers.

Christine van der Leeuw: Blood, bones and brains; peripheral biological endophenotypes and their structural cerebral correlates in psychotic disorder. Supervisor: Prof.dr. J. van Os; Co-supervisor: Dr. M. Marcelis.

Sanne Peeters: The Idle Mind Never Rests; functional brain connectivity across the psychosis continuum. Supervisor: Prof.dr. J. van Os; Co-supervisor: dr. M. Marcelis. 
Nick van Goethem: $\alpha 7$ nicotinic acetylcholine receptors and memory processes: mechanistic and behavioral studies. Supervisor: Prof.dr. H.W.M. Steinbusch; Co-supervisor: Dr. J. Prickaerts.

Nicole Leibold: A Breath of fear; a translational approach into the mechanisms of panic. Supervisor: Prof.dr. H.W.M. Steinbusch; Co-supervisors: Dr. K.R.J. Schruers / Dr. D.L.A. van den Hove. Renske Hamel: The course of mild cognitive impairment and the role of comorbidity. Supervisor: Prof.dr. F.R.J. Verhey; Co-supervisors: Dr. I.H.G.B. Ramakers / Dr. P.J. Visser.

Lucia Speth: Effects of botulinum toxin A injections and bimanual task-oriented therapy on hand functions and bimanual activities in unilateral Cerebral Palsy. Supervisors: Prof.dr. J. Vles; Prof.dr. R. Smeets; Co-supervisor: Dr. Y. Janssen-Potten, Adelante Hoensbroek.

Yuan Tian: The effects of Lutein on the inflammatory pathways in age-related macular degeneration (AMD). Supervisors: Prof.dr. C. Webers; Prof.dr. A. Kijlstra, WUR; Co-supervisor: Dr. M. Spreeuwenberg; Dr. H. Tange.

Peggy Spauwen: Cognition and Type 2 diabetes; the interplay of risk factors. Supervisors: Prof.dr. F. Verhey; Prof.dr. C. Stehouwer; Co-supervisor: Dr. M. van Boxtel

Marc Hilhorst: Crescentic glomerulonephritis in ANCA associated vasculitis. Supervisors: Prof.dr. J. Cohen-Tervaert; Co-supervisor: Dr. P. van Paassen

Martin Gevonden: The odd one out: exploring the nature of the association between minority status and psychosis. Supervisors: Prof.dr. J-P. Selten; Prof.dr. J. Booij, Uva; Prof.dr. I. Myin-Germeys

Bart Biallosterski: Structural and functional aspects of sensory-motor interaction in the urinary bladder. Supervisors: Prof.dr. Ph. Van Kerrebroeck; Prof.dr. S. De Wachter, UvAntwerpen; Co-supervisors: Dr. G. van Koeveringe; Dr. M. Rahnama'i.

Alexandra König: The use of information and communication technologies (ICT) for the assessment of patients with Alzheimer's Disease and related disorders. Supervisors: Prof.dr. F. Verhey; Prof.dr. Ph. Robert, Nice, Fr; Co-supervisors: Dr. P. Aalten; Dr. R. David, Nice. Fr.

Michelene Chenault: Assessing Readiness for Hearing Rehabilitation. Supervisors: Prof.dr. M.P.F. Berger; Prof.dr. B. Kremer; Co-supervisor: Dr. L.J.C. Anteunis.

Anand Vinekar: Retinopathy of Prematurity. Recent advances in tele-medicine screening, risk factors and spectral domain optical coherence tomography imaging. Supervisor: Prof.dr. C.A.B. Webers; Co-supervisor: Dr. N.J. Bauer

Fleur van Dooren: Diabetes and Depression: exploring the Interface between Pathophysiological and Psychological factors. Supervisors: Prof.dr. F.R.J. Verhey; Prof.dr. J.K.L. Denollet, UvT; Prof.dr. F. Pouwer, UvT; Co-supervisor: Dr. M.T. Schram.

Gabriëlla Pons van Dijk: Taekwondo and physical fitness components in middle-aged healthy volunteers; the Sekwondo study. Supervisors: Prof.dr. J. Lodder; prof.dr. H. Kingma; Co-supervisor: Dr. A.F. Lenssen.

Yara Pujol-Lopez: Development and psycho neuro immunological mechanisms in depression. Supervisor: Prof.dr. H.W.M. Steinbusch; Co-supervisors: Dr. G. Kenis; Dr. Aye Mu Myint, München. 